\title{
Sensing Characteristics of Core and Cladding Modes in Conventional Single Mode Fibre and Photonic Crystal Fibre
}

\author{
By
}

Chengkun Chen (M.A.Sc., M.Sc., B. Sc.)

\author{
A thesis submitted to \\ The Faculty of Graduate Studies and Research \\ in partial fulfilment of \\ the degree requirements of \\ Doctor of Philosophy \\ Ottawa-Carleton Institute for \\ Electrical and Computer Enginecring \\ Department of Electronics \\ Carleton University \\ Ottawa, Ontario, Canada \\ December 2008
}

Copyright $(2008$ - Chengkun Chen 
Library and

Archives Canada

Published Heritage

Branch

395 Wellington Street

Ottawa ON K1A ON4

Canada
Bibliothèque et

Archives Canada

Direction du

Patrimoine de l'édition

395, rue Wellington

Ottawa ON K1A ON4

Canada
Your file Votıe référence

ISBN: 978-0-494-52064-2

Our file Notre référence

ISBN: 978-0-494-52064-2
NOTICE:

The author has granted a nonexclusive license allowing Library and Archives Canada to reproduce, publish, archive, preserve, conserve, communicate to the public by telecommunication or on the Internet, loan, distribute and sell theses worldwide, for commercial or noncommercial purposes, in microform, paper, electronic and/or any other formats.

The author retains copyright ownership and moral rights in this thesis. Neither the thesis nor substantial extracts from it may be printed or otherwise reproduced without the author's permission.
AVIS:

L'auteur a accordé une licence non exclusive permettant à la Bibliothèque et Archives Canada de reproduire, publier, archiver, sauvegarder, conserver, transmettre au public par télécomm:ınication ou par l'Internet, prêter, distribuer et vendre des thèses partout dans le monde, à des fins commerciales ou autres, sur support microforme, papier, électronique etjou autres formats.

L'auteur conserve la propriété du droit d'auteur et des droits moraux qui protège cette thèse. $\mathrm{Ni}$ la thèse ni des extraits substantiels de celle-ci ne doivent être imprimés ou autrement reproduits sans son autorisation.
In compliance with the Canadian

Privacy Act some supporting forms may have been removed from this thesis.

While these forms may be included in the document page count, their removal does not represent any loss of content from the thesis.
Conformément à la loi canadienne sur la protection de la vie privée, quelques formulaires secondaires ont été enlevés de cette thèse.

Bien que ces formulaires aient inclus dans la pagination, il n'y aura aucun contenu manquant.

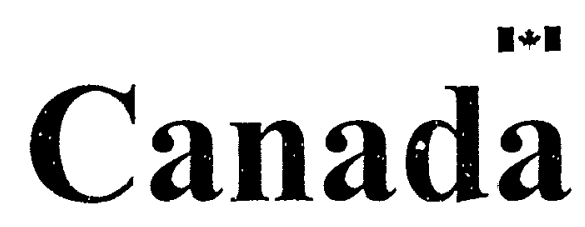




\section{Abstract}

Optical fibre sensors are widely used for strain, temperature, surrounding refractive index (SRI) and bending monitoring as well as for gyroscopes. Fibre Bragg gratings (FBG) have been widely used in telecommunications and sensors for temperature, strain and structural health monitoring. A single FBG only has one strong core mode (Bragg) resonance in its transmission spectrum or reflection spectrum, which is sensitive to both temperature and strain perturbations or even to SRI changes if the fibre has a very thin cladding layer. Therefore it is hard to use a single FBG to sense cither the temperature or strain without ambiguity. To distinguish temperature and strain effects, two Fr. is or one FBG and one long period grating need to be used; it brings the complexity for grating fabrication and sensor applications.

By writing the FBG in standard single mode fibre (SMF) with a weakly tilted angle between $3-10^{\circ}$, now in the transmission spectrum there is a strong core mode resonance but there are also cladding mode resonances because of the core mode coupling to the cladding modes. While the core mode resonance is sensitive to temperature and strain perturbations, the cladding mode resonances are sensitive to temperature, strain and SRI perturbations. The core mode resonance and the cladding mode resonances have the same temperature sensitivities. So by tracking the core mode resonance and cladding mode resonances, the ismperature effect can be removed and temperature compensated strain and SRI sensors can be developed. Furthermore by coating the fibre with a thin (20-30 
$\mathrm{nm}$ ) gold layer, a surface plasmon polariton can be excited by certain cladding modes. This plasmon resortance is very sensitive to SRI and can be used as a biomedical sensor for bio-molecular reactions occurring at the metal surfacc.

In the thesis, novel tilted FBG sensors have been developed for strain, temperature and index sensing applications using conventional SMF fibres. The differential strain sensitivity between the core and cladding modes in SMF-28 fibre are theoretically derived and they are well matched with the experiment result. The differential strain, temperature and SRI sensitivities in the SMF fibre with different cladding layer diameters have been analyzed and tested experimentally.

To further explore the characteristics of cladding modes in multilayer step-index fibre especially the fibre with a thin metal coating, a new vectorial mode solver, which can solve the lossless, lossy and amplifying optical fibres, has been developed. The coupling coefficients between the core mode and cladding modes are calculated for the fibre with FBG and tilted FBG. The results give a good explanation why the surface plasmon polariton can be excited in a gold coated fibre with tilted FBG.

Finally strong FBGs are written in a germanium/fluorine co doped core photonic crystal fibre (PCF). A iimited number of strong cladding mode resonances are obtained with a FBG. The strain, temperature and refractive index sensitivities of core and cladding mode resonances in this PCF have been determined experimentally. In addition to the usual core and cladiding modes, this PCF has a cladding mode whose optical field is confined by the air-hole region, making this mode insensitive to the surrounding refractive index changes, just like the core-guided mode. The main advantage of the PCF short periud gratings in sensing applications resides in the presence of the SRI-insensitive iv 
cladding mode, whose differential resonance shift can be used to sense axial strain independently of temperature and regardless of the material in which the sensor is placed, including liquids, epoxies or structural materials such as concrete.

As the multilayer fibre mode solver could be integrated into a commercial optical CAD software, the fibre sensors based on TFBGs in conventional fibres and FBGs in PCF are suitable for real industrial sensing applications. 
To my wife Fangyu and my daughter Katie 


\section{Acknowledgements}

I would like to express my deepest gratitude to my supervisor Prof. Jacques Albert for his constant guidance, inspiration, advice, and encouragement throughout this thesis project.

I am grateful to my thesis committee members: Prof. Tom Smy, Prof. Pierre Berini, Prof. Wojtek J. Bock, Prof. Robert Gauthier and Prof. Fred Nitzsche for their constructive feedback contributed to this thesis.

I would also like to thank my colleagues Ms. Albane Laronche, Dr. Tuan Guo, Mr. Lingyun Xiong and Ms. Yanina Y. Shevcheko for their help and cocperation, and Dr. Peter Krug for proofreading part of this manuscript and providing some valuable suggestions.

I would also like to thank Dr. Steve Dods and Dr. Richard Zhang of Optiwave Systems Corporation for fruitful discussions on the fibre mode in step-index optical fibres.

I would also like to take this opportunity to thank my wife Fangyu for her continued supporl, understanding, love and patience during my studies and research.

Finally, I acknowledge LxSix Photonics, NSERC, CIPI, OGSST, Carleton University and The Gary S. Duck Scholarship for their financial support during this work. 


\section{Table of Contents}

$\begin{array}{lll}\text { Abstract } & \text { iii }\end{array}$

Acknowledgements vii

Table of Contents viii

List of Tables $\quad$ xiii

List of Figures $\quad$ xiv

List of Acronyms $\quad \mathrm{xx}$

Chapter 1 Introduction $\quad 1$

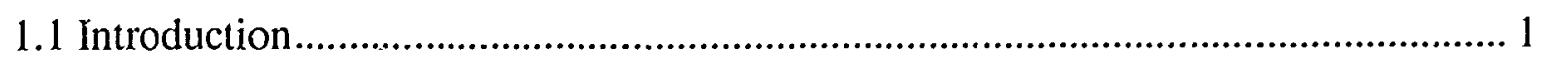

1.1.1 Optical Fibres and Fibre Modes................................................................. 1

1.1.2 Mode Couplings and Fibre Bragg Grating....................................................... 2

1.1.3 Tilted Fibre Bragg Grating............................................................................. 3

1.2 Organization of the Thesis ............................................................................. 3

Chapter 2 Literature Review and Thesis Objective 5

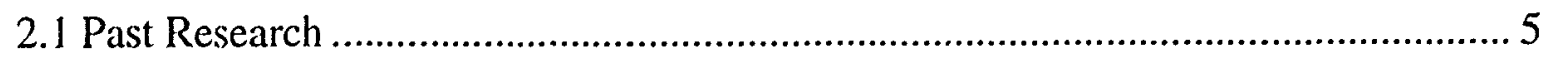

2.1.1 Analysis of Optical Fibre Mode..................................................................... 5

2.1.2 Mode Ccuplings in Optical Fibres... ................................................................. 6

2.1.3 Tilted Fibre Bragg Gratings in Single Mode Optical Fibres for Sensing Application: ................................................................................................. 6

2.1.4 Fibre Bragg Gratings in Photonic Crystal Fibres............................................ 7

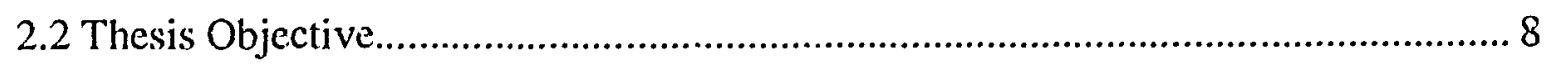

viii 
Chapter 3 Optical Fibre Modes in Multilayer Step-Index Fibres with Lossless, Lossy and Gain Media

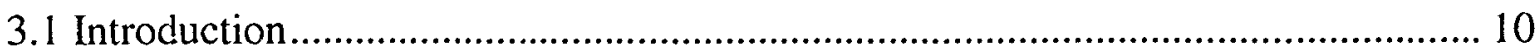

3.2 Maxwell's Equations in Cylindrical Coordinates ............................................. 12

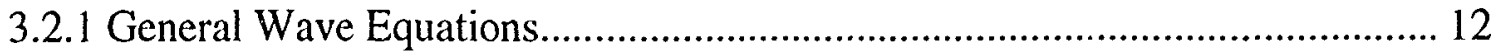

3.2.2 General Solutions for HE/EH, TE, and TM Waves.................................. 13

3.3 Wave Equations for Step-Index Fibres ..................................................... 15

3.4 Hybrid Modes in Multilayer Step-Index Optical Fibres ..................................... 16

3.4.1 Dispersion Equation of Hybrid Modes ..................................................... 16

3.4.2 The Fields of Hybrid Modes ................................................................. 22

3.5 Transverse Modes in Multilayer Step-Index Optical Fibres................................ 25

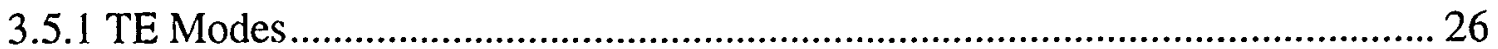

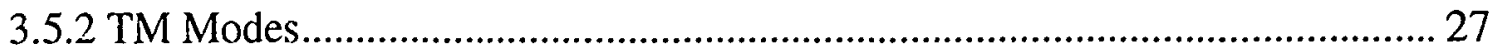

3.6 Linear Polarization Modes in Multilayer Step-Index Optical Fibres..................... 28

3.7 Solving the Dispersion Equations for Lossless Optical Fibres............................. 30

3.8 Solving the Dispersion Equations for Lossy and Gain Optical Fibres .................. 30

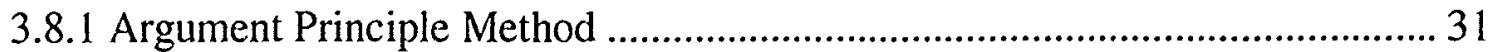

3.8.2 Derivative of Dispersion Equations of Hybrid Modes ............................... 32

3.8.3 Algorithm for Computing the Derivative of the Transfer Matrices................. 35

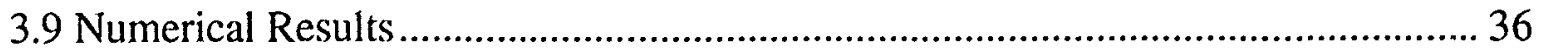

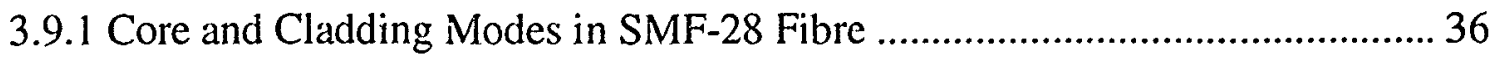

3.9.2 Cladding Modes in SMF Fibre with Nano-Size Metal Coating .................... 38

3.9.3 The Plasmon Mode in Thinned SMF fibre with a Thin Gold Coating ........... 41

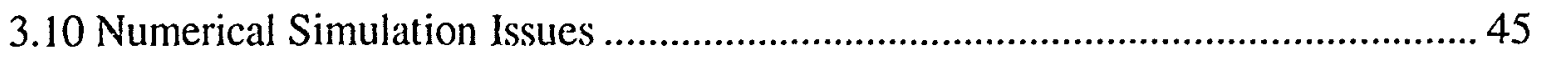

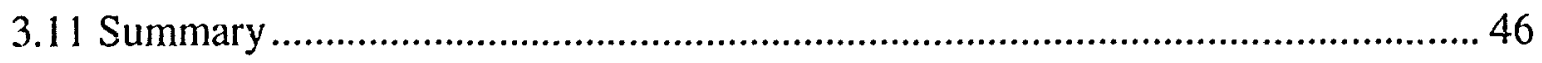

Chapter 4 Mode Couplings in Optical Fibres with Loss'ess, Lossy and Gain Media

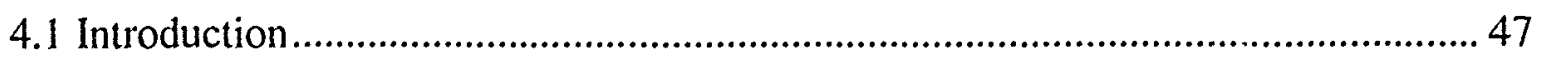

4.2 The Electric Field Distributions in Multilayer Step-Index Fibre.......................... 48 
4.3 Cladding Mode Couplings with Core Mode ...................................................... 50

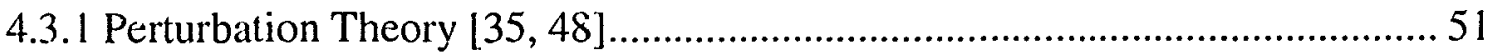

4.3.2 Core and Cladding Mode Coupling Coefficients in Fibres with FBG.............53

4.3.3 Core and Cladding Mode Coupling Coefficients in Fibres with TFBG ..........56

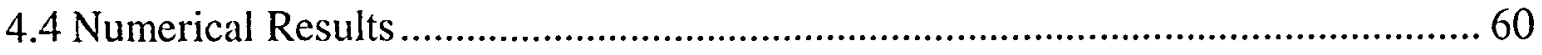

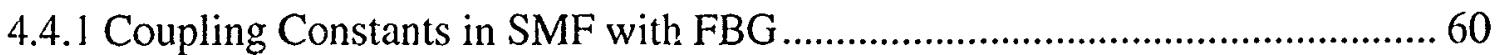

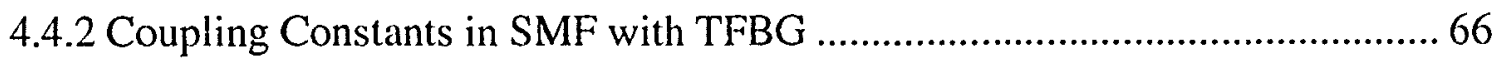

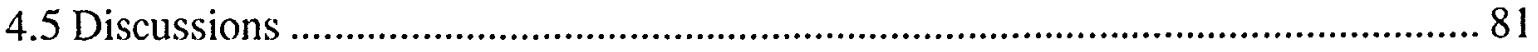

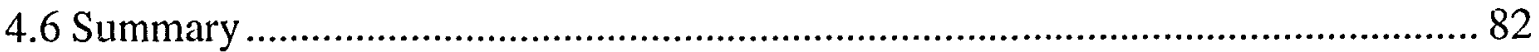

Chapter 5 Sensing Characteristics of Core and Cladding Modes in Single Mode

Fibres $\quad 84$

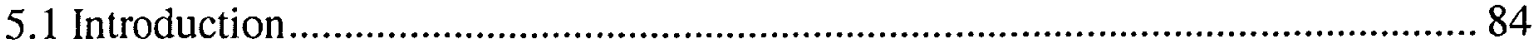

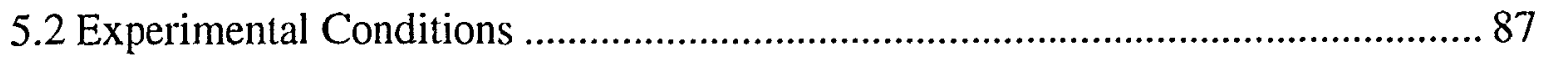

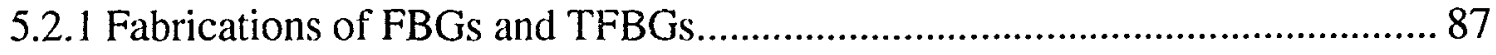

5.2.2 The Transmission Spectrum Characteristics of FBG and TFBG in Single Mode

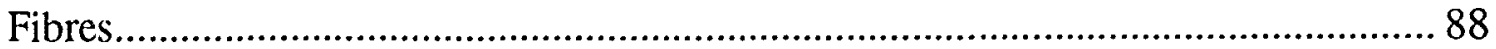

5.2.3 Polarization Dependence of the SMF-28 Mode Resonances.......................... 94

5.3 Analysis of Core and Cladding Mode Sensitivities of SMF-28 Fibres in TFBG with

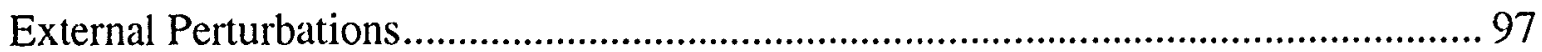

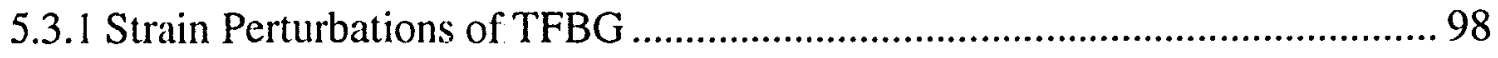

5.3.2 Temperature Perturbations of TFBG ..................................................... 99

5.3.3 Surrounding Refractive Index Perturbations of TFBG ................................ 99

5.4 The Sensitivity of the Core and Cladding Modes of SMF-28 Fibres .................. 101

5.4.1 Differential Strain Sensitivity of TFBG............................................... 101

5.4.2 Differential Temperature Sensitivity of TFBG .......................................... 105

5.4.3 Surrounding Refractive Index Sensitivity............................................. 107

5.5 The Sensitivity of the Core and Cladding Modes of SMFs with Thinned Cladding

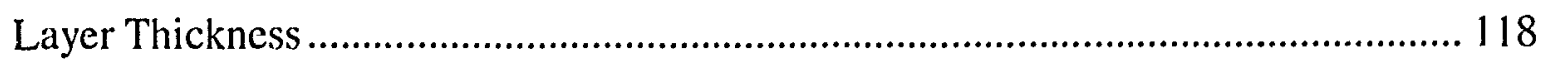

5.5.1 Differential Strain and Temperature Sensitivitics of TFBG ....................... 118

5.5.2 Surrounding Refractive Index Sensitivity............................................ 126 
5.6 Discussion

5.7 Summary

Chapter 6 Sensing Characteristics of Core and Cladding Modes in Photonic Crystal

Fibres

6.1 Introduction. 141

6.2 Experimental Conditions 143

6.3 Core and Cladding Mode Resonances in PCF Fibres. 145

6.3.1 The Spectrum Characteristics of FBG in PCF Fibre 145

6.3.2 Simulations of the PCF Modes 148

6.3.3 Polarization Dependence of the PCF Mode Resonances. 149

6.4 The Sensitivity of the Core and Cladding Modes of PCFs.. 151

6.4.1 Strain and Temperature Sensitivity. 151

6.4.2 Surrounding Refractive Index Sensitivity. 157

6.4.3 Bending Sensitivity 160

6.5 Discussions 162

6.6 Summary 165

Chapter 7 Conclusions and Future Work

7.1 Original Contributions 167

7.2 Significance of Work. 168

7.3 Future Work 169

Bibliography

Appendix A: The Power in Multilayer Step-Index Fibre with Lossy and Gain Media

B1. Patents 183

B2. Referred Journal Papers 183

B3. Book Chapters 184 


\section{List of Tables}

Table 3.1: The effective index of different fibre modes in SMF-28 fibre .................... 37

Table 3.2: The effective index of different fibre modes in SMF-28 fibre ...................... 39

Table 3.3: The effective index of different fibre modes in thinned SMF fibre ............. 43

Table 6.1: Summary of transmission resonance properties.................................... 147

Table 6.2: Core and Cladding Mode Temperature and Strain Sensitivities ................. 152

xiii 


\section{List of Figures}

Figure 3.1: The cross-section of $m$ layer step-index fibre ............................................. 16

Figure 3.2: The cross-section of SMF-28 fibre............................................................ 36

Figure 3.3: The cross-section of SMF fibre with $36 \mathrm{~nm}$ metal coating ............................ 39

Figure 3.4: The normalized azimuthal magnetic fields of cladding modes: (a) oud modes,

(b) even modes

Figure 3.5: The azimuthal magnetic fields of cladding modes: (a) odd modes; (b) even modes from reference [4.4]; (c) odd modes, and (d) even modes are simulated............... 41

Figure 3.6: The cross-section of thinned SMF fibre with $25 \mathrm{~nm}$ gold coating .................. 42

Figure 3.7: The normalized magnetic fields of slasmon modes with different SRI: (a)

longitudinal component; (b) radial component, and (c) azimuthal component................ 44

Figure 4.1: Electric field distributions in SMF-28 fibre............................................... 50

Figure 4.2: Configuration of a single-sided tilted grating: (a) for an $x$-tilted grating, (b) for a $y$-tilted grating.

Figure 4.3: Coupling constant $\kappa_{1 m-11}$ divided by $\sigma(z)$ for the $168 v=1$ cladding modes in a SMF fibres.

Figure 4.4: Coupling constant $\kappa_{1 m-11}$ divided by $\sigma(z)$ for $v=1$ cladding modes in the SMF-28 fibre.

Figure 4.5: Comparing the coupling constant $\kappa_{1 m-1 \mid}$ divided by $\sigma(z)$ for $v=1$ cladding modes in a small core SMF fibre and SMF-28 fibre.

Figure 4.6: The transmission spectrum of a FBG in the SMF-28 fibre and simulated coupling constant $\kappa_{1 m-11}$ divided by $\sigma(z)$ for $v=1$ cladding modes in the SMF-28 fibre. 64 Figure 4.7: Coupling constant $\kappa_{1 m-11}$ divided by $\sigma(z)$ for $v=1$ cladding modes in a SMF28 fibre with $20 \mathrm{~nm}$ gold coating. 65 
Figure 4.8: AC coupling constant $2 g_{v m-11} / \sigma(z)$ for cladding modes with (a) $v=1$, $\theta=0^{\circ}$, (b) $v=2, \theta=5^{\circ}$, (c) $v=3, \theta=7.5^{\circ}$.

Figure 4.9: AC coupling constant $2 g_{v^{\prime} m-11} / \sigma(z)$ for cladding modes with (a) $v=1$, $\theta=0^{\circ}, 4^{\circ}$ and $6^{\circ}$ for HE modes, (b) $v=1, \theta=0^{\circ}, 4^{\circ}$ and $6^{\circ}$ for EH modes, (c) $v=2$, $\theta=4^{\circ}$ and $6^{\circ}$, (d) $v=3, \theta=4^{\circ}$ and $6^{\circ}$ (e) $v=4, \theta=4^{\circ}$ and $6^{\circ}$ in SMF-28 fibre.

Figure 4.10: The transmission spectrum of a TFBG in the SMF-28 fibre with a tilted angle $\theta=4^{\circ}$ and simulated $\mathrm{AC}$ coupling constant $2 g_{v^{\prime m-11}} / \sigma(z)$ for core and cladding modes with $v=1$ and 2 in the SMF-28 fibre.

Figure 4.11: The transmission spectrum of a TFBG in the SMF-28 fibre w: th a tilted angle $\theta=4^{\circ}$ and simulated AC coupling constant $2 g_{v m-11} / \sigma(z)$ for core and cladding modes with $v=1$ and 2 in the SMF-28 fibre.

Figure 4.12: $\mathrm{AC}$ coupling constant $2 g_{v m-11} / \sigma(z)$ for cladding modes (a) $\mathrm{HE}_{\mathrm{Im}}$ modes: $\theta=0^{\circ}, 4^{\circ}$ and $6^{\circ}$, (b) $\mathrm{EH}_{1 \mathrm{~m}}$ modes: $\theta=0^{\circ}, 4^{\circ}$ and $6^{\circ}$, (c) $\mathrm{HE} 2 \mathrm{~m}$ modes: $\theta=4^{\circ}$ and $6^{\circ},(\mathrm{d})$ $\mathrm{EH}_{2 \mathrm{~m}}$ modes: $\theta=4^{\circ}$ and $6^{\circ}$, in SMF-28 fibre with $20 \mathrm{~nm}$ gold coating and $\mathrm{n}_{4}=1.33 \ldots \ldots .77$ Figure 4.13: (a) The transmission spectrum of TFBG with a $15 \mathrm{~nm}$ gold coating immersed in a sucrose solution with $\mathrm{n}_{\mathrm{D}}=1.4378$ [45], (b) AC coupling constant $2 g_{v m-11} / \sigma(z)$ and mode propagation loss for cladding $\mathrm{HE}_{1 \mathrm{~m}}$ with $\theta=6^{\circ}$ and $\mathrm{n}_{\mathrm{S}}=1.415$, (c) Mode loss envelope of HElm modes with different SRI. 79

Figure 4.14: (a) Simulated dependence of the plasmon peak wavelength $\left(\lambda_{\text {plasmonin }}\right)$ and corresponding cladding mode effective index on the SRI, (b) measured dependence of the resonance peak wavelength $\left(\lambda_{\mathrm{P}}\right)$ and correspending cladding mode effective index on the

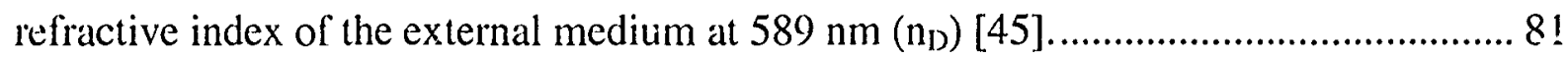

Figure 5.1: (a) TFBG schematic; (b) the transmission spectrum of a TFBG. ................. 86 Figure 5.2: The top view of setup for TFBG written ............................................. 88 Figure 5.3: The transmission spectra of FBG and $4^{n}$ tilted FBGs written in SMF-28 single mode fibres: (a) FBG, (b) TFBG written by $248 \mathrm{~nm}$ KrF excimer laser light; (b) TFBG written by $193 \mathrm{~nm}$ ArF excimer laser light. 90

Figure 5.4: The experimental and simulated transmission spectrum of $4^{\circ}$ tilted FBG.... 92 
Figure 5.5: The simulated transmission spectrum by using hybrid modes and the experimental transmission spectrum of $4^{\circ}$ tilted FBG.

Figure 5.6: The PDLS of core and cladding modes: (a) TFBG written by $248 \mathrm{~nm} \mathrm{KrF}$ excimer laser light; (b) TFBG written by $193 \mathrm{~nm}$ ArF excimer laser light. 96

Figure 5.7: The Transmission Spectra of FBGs in PCF with Different Polarization States: (a) TFBG written by $248 \mathrm{~nm}$ KrF excimer laser light; (b) TFBG written by $193 \mathrm{~nm}$ ArF excimer laser light.

Figure 5.8: The transmission spectrum of $4^{n}$ tilted FBG and differential wavelength shifts due to strain changes: (a) written by $193 \mathrm{~nm}$ ArF excimer laser light; (b) written by 248 $\mathrm{nm} \mathrm{KrF}$ excimer laser light. 102

Figure 5.9: Differential wavelength shift of individual resonances for $4^{\circ}$ tilted FBGs written by ArF and KrF excimer laser light. 103

Figure 5.10: The relative wavelength shift of LP1 26 mode to LPO 1 mode for different strains with maximum temperature error. 104

Figure 5.11: The transmission spectrum of $4^{n}$ tilted FBG and differential wavelength shift due to the temperature changes: (a) written by $193 \mathrm{~nm}$ ArF excimer laser light 11; (b) written by $248 \mathrm{~nm} \mathrm{KrF}$ excimer laser light. 106

Figure 5.12: Comparison of the differential wavelength shifts of a single cladding mode resunance for a $4^{\circ}$ tilted $\mathrm{FBG}$ at wavelength $\sim 1536 \mathrm{~nm}$ duc to strain and temperature perturbations

Figure 5.13: Typical experimental TFBG transmission spectrum (CORNING SMF28 fibre, $\theta=6^{\circ}$ ) measured in air.

Figure 5.14: (a) Several measurements with various refractive indices of the outer medium, near the Bragg resonance. (b) Same spectra as (a) but zooming in on a particular resonance near $1535.5 \mathrm{~nm}$.

Figure 5.15: Experimental shift in the distance of a cladding mode resonance from the Bragg wavelength as a function of the refractive index of a sugar solution at $589 \mathrm{~nm} .111$ Figure 5.16: Change in wavelength separation from the Bragg wavelength for four different cladding modes as a function of the refractive index of calibrated liquids Filled 
symbols $=$ experimental data at room temperature $\left(23^{\circ} \mathrm{C}\right)$; Open symbols $=$ Experimental data at $50{ }^{\circ} \mathrm{C} ;$ Lines $=$ simulations.

Figure 5.17: Experimental transmission spectrum of a TFBG with a tilt angle of 10 degrees, $\left(\lambda_{\text {Bragg }}=1566.810 \mathrm{I} \mathrm{m}\right)$.

Figure 5.18: Shift of a resonance near $1500 \mathrm{~nm}$ for various external media (solid line: SRI=1.0, dotted line: $S R I=i \quad$ 758, dashed line: $S R I=1.3250$, all values for $1500 \mathrm{~nm}$ )) 115 Figure 5.19: Measured refractive index from the average of the predictions based on the shifts of 4 resonances $(-8 \mathrm{~nm},-14 \mathrm{~nm},-23 \mathrm{~nm}$, and $-28 \mathrm{~nm})$ versus actual refractive index.

Figure 5.20: 'The transmission spectra of weakly TFBG: (a) SMF-28 fibre $d=125 \mu \mathrm{m}$ and $\theta=4^{\circ}$; (b) Corning experimental thin cladding layer fibre $d=50 \mu \mathrm{m}$ and $\theta=6^{\circ}$; (c) Thin cladding layer by HF etching from SMF-28 fibre $d=50 \mu \mathrm{m}$ and $0=4^{\circ}$.

Figure 5.21: The relative wavelength shift of cladding mode resonances to the Bragg resonance of TFBGs (a) with standard SMF-28 fibre $d=125 \mu \mathrm{m}$ and $\theta=4^{\circ}$ due to longitudinal strain and temperature Perturbations, $(b)$ with thin cladding layer $d=50 \mu \mathrm{m}$ and $0=6^{\circ}$ due to longitudinal strain and temperature perturbations.

Figure 5.22: Differential wavelength shift of individual resonances for thin and regular fibres near $3860 \mu \varepsilon$.

Figure 5.23: Comparison of differential wavelength shifts of cladding mode resonances for a $4^{\circ}$ tilted FBG in SMF-28 fibre and a $6^{\circ}$ tilted FBG in Corning experimental $50 \mu \mathrm{m}$ diameter thin cladding fibre with $\sim 22 \mathrm{~nm}$ away from their Bragg resonanccs due to strain and temperature perturbations. 125 Figure 5.24: The transmission spectra of weakly TFBG: (a) SMF-28 fibre $d=125 \mu \mathrm{m}$ and $\theta=4^{\circ}$; (b) Corning experimental thin cladding layer fibre $\mathrm{d}=50 \mu \mathrm{m}$ and $0=4^{\circ}$.

Figure 5.25: Experimental relative wavelength shifts of cladding mode resonances to the Bragg resonance of FBG with surrounding refractive index $\left(n_{D}\right)$ changes $(a)$ in $S M F-28$, (b) in $50 \mu \mathrm{m}$ diameter SMF. 130

Figure 5.26: The relative wavelength shifts of cladding mode resonances to the Bragg resonance of TFBG with surrounding refractive index $\left(n_{D}\right)$ changes (a) in SMF-28, (b) in $50 \mu \mathrm{m}$ diameter SMF. 130

xvii 
Figure 5.27: (a) Schematic diagram of a TFBG with a misaligned fusion for vibration measurement and before and after photographs of the fusion splice. (b) TFBG Spectra before and after misaligned junction, (c) Harmonic oscillating response of the sensor system following an impulse excitation [63].

Figure 5.28: (a) Spectra of $6^{\circ} \mathrm{TFBG}$ versus $\mathrm{RI}$ at a fixed temperature $\left(25^{\circ} \mathrm{C}\right),(\mathrm{b})$ Normalized power response of $6^{\circ} \mathrm{TFBG}$ versus $\mathrm{XI}$ at a fixed temperature $\left(25^{\circ} \mathrm{C}\right.$ : closed symbols), and other temperatures (40 and $55^{\circ} \mathrm{C}$ : open symbols) [64]. 134

Figure 5.29: (a) The transmission spectrum of a TFBG with tilt angle $0=4^{\circ}$ with SRI higher than fibre cladding refractive index: (b) The transmission spectrum of one selected radiation mode resonance for SRI higher than the fibre cladding refractive index; (c) Peak-to-peak value response of the selected radiation mode resonance in different SRI

Figure 5.30: The transmission spectrum of TFBGs in a sucrose solution with $n_{D}=1.4378$ : (a) with a $15 \mathrm{~nm}$ gold coating. The bracket indicates the peak position of the anomalous resonance: (b) without gold coating; (c) dependence of the resonance peak wavelength $\left(\lambda_{P}\right)$ and corresponding cladding mode effective index on the refractive index of the external medium at $589 \mathrm{~nm}\left(\mathrm{n}_{\mathrm{D}}\right)$ [45].

Figure 6.1: The cross section of the photonic crystal fibre used in the experiment (outside fibre diameter $=125 \mu \mathrm{m})$. The doped core is not visible in these photographs............ 144 Figure 6.2: The transmission and reflection spectra of a $1 \mathrm{~cm}$-long FBG in PCF fibre. 145 Figure 6.3: The electric field intensities of the core mode and cladding modes, the insets show an enlarged view of the core area. 147

Figure 6.4: The polarization dependent loss of core and cladding modes. 149 Figure 6.5: The Transmission Spectra of FBGs in PCF with Different Polarization States 150

Figure 6.6: The core mode resonance shift under axial strain perturbations 151 Figure 6.7: The strain sensitivity of the core mode 152

Figure 6.8: The relative wavelength shifts of cladding mode resonances relative to the Bragg resonance under axial strain perturbations. 
Figure 6.9: The strain sensitivity differences of core and cladding modes in the PCF fibre and SMF-28 fibre 154

Figure 6.10: The core mode resunance shift under temperature perturbations............... 154

Figure 6.11: The temperature sensitivity of the core mode 155

Figure 6.12: The relative wavelength shifts of cladding mode resonances relative to the

Bragg resonance of FBG under temperature perturbations. 156

Figure 6.13: The relative wavelength shifts of cladding mode resonances relative to the Bragg resonance, with respect to surrounding refractive index $\left(n_{\mathfrak{f}}\right)$ changes. ............... 157

Figure 6.14: The transmission spectra of FBG-PCF immersed in higher index oil. ...... 159

Figure 6.15: The transmissicn spectra of the FBGs in PCF with different bending

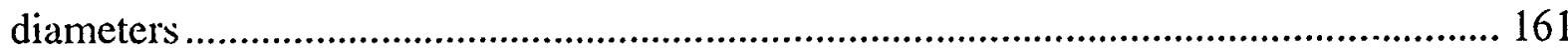

Figure 6.16: The bended $(D=9 \mathrm{~cm})$ FBGs in PCF with different polarization states .... 161 Figure 6.17: (a) The PCF fibre cross section [28]; (b) The spectra of FBGs in PCF [28].

Figure 6.18: The transmission and reflection spectra of a $1 \mathrm{~cm}-\mathrm{long}$ TFBG in PCF fibre. 


\section{List of Acronyms}

$\begin{array}{ll}\text { SMF } & \text { Single Mode Fibre } \\ \text { FBG } & \text { Fibre Bragg Grating } \\ \text { TFBG } & \text { Tilted Fibre Bragg Grating } \\ \text { LP } & \text { Linearly Polarized } \\ \text { TE } & \text { Transverse Electric } \\ \text { TM } & \text { Transverse Magnetic } \\ \text { LPG } & \text { Long Period Grating } \\ \text { PDI } & \text { Polarization Dependent Loss } \\ \text { SRI } & \text { Surrounding Refractive Index } \\ \text { UV } & \text { Ultra-Violet } \\ \text { FWHM } & \text { Full Width at Half Maximum } \\ \text { RIU } & \text { Refractive Index Units } \\ \text { PCF } & \text { Photonic Crystal Fibre }\end{array}$

$\mathrm{xx}$ 


\section{Chapter 1}

\section{Introduction}

\subsection{Introduction}

\subsubsection{Optical Fibres and Fibre Modes}

Fibre optical communications bring a revolution for the telecommunications. Without fibre optical communication, our life would be totally different today, high speed data communication and high speed internets are impossible. Optical fibre is a fundamental transmission medium for fibre optical communications, they take lots of advantages over the coaxial cable and hollow metal waveguides such as low loss (around $0.2 \mathrm{~dB} / \mathrm{km}$ at $1550 \mathrm{~nm}$ ), low cost (several cents/m), snall size (diameter around several hundred micrometer) and extremely high bandwidth allowing very high bitrates (up to $160 \mathrm{Gbit} / \mathrm{s}$ per channel) [1]. The optical fibre not only can be a medium for fibre optical communications but it is also widely used in optical sensors as early as 1970's [2].

To understand the light transmission in an optical fibre, the field distributions in the cross-section of the fibre and the light transmission speed in the fibre need to be known, therefore the fibre mode need to be solved. Different fibres will support different fibre modes, based on how many core modes in the fibre, fibres can be classified as single mode fibre (SMF) and multimode fibre (MMF). If the fibre only supports one core mode it is called a SMF and if the fibre supports multiple core modes then it is called MMF. Except the core mode, fibres also can support cladding modes, leaky mules and radiation 
modes depending on the core, clatding and surrounding medium index contrast. The fibre alst :an be classified by the core and cladding refractive index contrast as weakly guided or strongly guided as well as step-index fibre or graded-index fibre. As SMFs are normally used in Long Haul communications, the MMFs are used in Metro Networks. For the sensor applications, there are other special fibres such as D fibre, double cladding fibre, photonic crystal fibre (PCF) etc. for specific sensing purposes.

\subsubsection{Mode Couplings and Fibre Bragg Grating}

isere are lots of optical devices that are using the principle of mode couplings such as optical couplers, optical mode converters, grating based optical filters and etc. Among those devices, lots of them are fibre Bragg grating (FBG) based for both telecommunication and sensor applications. The FBG was invented in 1978 by Hill et al [3]. FBGs can be easily fabricated since the side ulcra-violet (UV) writing technique was invented [4], and especially after the phase mask was invented [5]. FBGs can selectively reflect light at a certain wavelength when it meets the Bragg condition. The FBG has several advantages compared with the bulk device such as compact in-fibre geometry, cost efficiency, low insertion loss, and flexibility of achieving desired spectral profiles. Therefore, FBGs have been playing a key role in both fibre optic communication and fibre optic sensing system $[6,7]$.

Fibre Bragg grating (FBG) sensors have a wide range of applications such as pressure-strain sensors, temperature sensors. micro-bending sensors and external refractive index sensors [8-13]. As these optical sensors are inherently immune from 
electromagnetic interference and chemically inert, they are very attractive in biochemical applications and hazardous surroundings.

\subsubsection{Tilted Fibre Bragg Grating}

A tilted Fibre Bragg grating (TFBG) is a fibre Bragg grating with grating plane tilted at small angles relative to the fibre axis perpendicular. The TFBG couples light to core and cladding modes propagating backwards [14]. The resonant wavelength for each mode depends differentially on external perturbations and thus can be used to make the fibre grating into a multi-parameter sensor. Using the core mode back reflection resonance as a reference wavelength in single mode fibres, the relative shift of the cladding mode resonances can be used to selectively measure perturbations such as strain or surrounding refractive index, and this independently of temperature.

\subsection{Organization of the Thesis}

Chapter 2 will describe some background on the fibre modes, mode coupling in the fibre with FBGs and tilted FBGs, the TFBG characteristics in conventional SMFs and their sensor applications and FBGs in photonic crystal fibre.

Chapter 3 develops the numerical method for accurately and efficiently analyzing the fibre mode in multilayer step index fibres. The analytical inverse matrices will be derived and different algorithm to rapidly solve the dispersion equations will be discussed. Some examples of different optical fibre structures will be presented and compared with examples from the literature. 
Chapter 4 focuses on the fibre mode couplings between the core mode and itself and also with cladding modes for sinusoidal index modulations in the fibre core layer with or without a small tilted angle. Full vectorial modes will be used to analyze mode couplings between the hybrid $\mathrm{HE}_{11}$ mode to hybrid $\mathrm{HE}_{11}$ mode and $\mathrm{HE}_{\mathrm{vm}} / \mathrm{EH}_{\mathrm{vm}}$ cladding modes in FBG and TFBG with a small tilted angle. Numerical results for TFBG with a gold coating will be discussed in detail and compared with experiment results.

Chapter 5 presents novel optical sensors based on TFBG in conventional SMFs for temperature-independent strain and refractive index sensing. The experimental temperature, strain and index sensitivities of TFBG with different cladding layer thickness are presented as well.

Chapter 6 describes the sensitivity characteristics of a strong FBG in PCF, the strain, temperature and refractive index sensitivities of core and cladding mode resonances in this PCF fibre will presented. There are several index sensitive cladding modes and one index insensitive cladding mode in PCF, which combined with the core mode make possible novel multi-parameter sensing applications for FBG in PCF.

Finally, conclusions from this research and suggestions for further research are presented in Chapter 7. 


\section{Chapter 2}

\section{Literature Review and Thesis Objective}

\subsection{Past Research}

\subsubsection{Analysis of Optical Fibre Mode}

There are several methods which can be used to solve the vectorial and scalar wave equations [15] to obtain the effective index of each mode and its field distributions. Probably the best choice for a general, quick algorithrn is the method developed by Yeh and Lindgren [16]. The method is similar to the transfer matrix method used for the analysis of planar waveguides [17]. Both methods use a matrix to express the fields at one side of a layer given their values at the other side, and the whole structure is then characterized by cascading the layers by matrix multiplication except that in the method of Yeh and Lindgren, a set of inverse matrices need to be calculated. The numerical calculation of inverse matrix sometimes will not be accurate especially when the matrix is close to singular. Recently it was found that the transfer matrix can be decomposed to two simple matrices, and inverse matrices of these simple matrices can be obtained analytically [18]. Based on that a commercial optical fibre mode solver has been developed by Optiwave Systems Corporation, but it is only for guided modes in lossless optical waveguides. 


\subsubsection{Mode Couplings in Optical Fibres}

There are lots of opual devices that are using the principle of mode couplings such as optical couplers, optical mode converters, grating based optical filters etc. Among those devices, many of them are optical long period gratings (LPG) and fibre Bragg gratings (FBG) based for both telecommunication and sensor applications. The theory for mode couplings in optical waveguides and DFB lasers was well developed [19] even before the FBG was invented in 1978 by Hill et al [3]. There are several methods that can be used to analyze the mode coupling in fibres. Among them, the coupled mode theory is the most developed and most used to analyze the LPGs and FBGs [20-22]. The most detailed work for calculating the coupling constant between modes are done by Erdogan et al [21]. Erdogan provides analytical solutions for the coupling constant for LPGs and FBGs, while Lees and Erdogan derive the formulation of coupling constants for tilted gratings including tilted LPG and TFBG [22]. All their work uses the $\mathrm{LP}_{01}$ core mode approximation, and they calculate the coupling constant between $\mathrm{LP}_{01}$ and $\mathrm{HE}_{\mathrm{vm}} / \mathrm{EH}_{\mathrm{vm}}$ cladding moded in 3 layer SMFs.

\subsubsection{Tilted Fibre Bragg Gratings in Single Mode Optical Fibres for Sensing Applications}

The concept of using cladding mode resonances from a TFBG was originally proposed by Laffont and Ferdinand [23]. They track the envelope of the resonances in the transmission spectrum when the SRI changes. As the SRI increases, high order cladding modes are cutoff and become leaky modes, when this occurs, in transmission spectrum, the area enclosed by the envelope of the resonances decreases, therefore the SRI changes 
correspond to the area changes. Therefore, the TFBG can be used as a refractive index sensor. The same method is also used for sensing when the SRI is higher than the fibre cladding refractive index [24]. While all cladding modes become radiation modes when the SRI is higher than the fibre cladding refractive index, the coupling strength to individual radiation modes changes markedly for different SRI. Using this effect the TFBG can sense SRI values higher than the refractive index of the cladding layer. In the work presented by Laffont and Ferdinand, TFBGs have large tilt angles $\left(8^{\circ}\right.$ to $\left.16^{\circ}\right)$ and each cladding mode resonance has different temperature sensitivity, making temperatureindependent multimodal sensors impossible.

\subsubsection{Fibre Bragg Gratings in Photonic Crystal Fibres}

Photonic crystal fibres (PCF) were first demonstrated in 1996 [25] and have been extensively explored for telecommunication and sensor applications [26]. In particular, solid core PCF fibres have rings of low refractive index inclusions, as air holes for example, along the radial direction, forming an equivalent inner cladding layer which has lower refractive index than the core and the pure silica outer cladding. In such structures, in addition to an endlessly single mode core mode and conventional cladding modes, there are cladding modes whose fields are confined only within the core and air-hole region $[27,28]$ and their optical fields cannot penetrate the outermost cladding layer to reach the surrounding medium. Those unique characteristics of PCF fibre cladding modes make them very attractive for sensor applications if the modes can be excited and measured in a controlled fashion. FBG can selectively excite cladding modes in special PCFs that have a germanium/fluorine co-doped core $[28,29]$ to increase their 
photosensitivity relative to undoped PCF fibres [30]. Apart from the photosensitivity issue, which can also be solved by using ultrafast laser sources [31,32], writing good quality FBGs in PCF fibre poses a big challenge due to scattering by the air-holes in the cladding layer [33]. This particular problem can be minimized by scanning the laser focus while rotating the fibre to find a path for the laser light towards the core [31], by collapsing the air hole region through tapering [31], and by filling the air-holes with index matching fluids [34]. All previous work in this field shows that cladding modes are insensitive to SRI meaning that all the modes involved were confined in the core and airhole regions.

\subsection{Thesis Objective}

A primary objective of the thesis is to develop novel optical sensors that are low cost, easy to fabricate, reliable and capable for multi-parameter sensor applications. The TFBG in conventional SMF-28 fibres and other SMFs is well matched to these requirements. SMFs are low cost, fabrication of FBGs in SMFs is a mature technology, the reliability of FBGs has been verified in telecommunication products and more importantly TFBG can excite sets of strong cladding mode resonances, therefore it is possible to achieve the multi-parameter sensing purpose. To increase the sensitivity of TFBG sensors, it is also possible to coat the fibres with specific dielectric or metal overlays to sensitive the fibres to gases or biomedical materials. To develop and optimize TFBG for these purposes several steps are needed:

(i) A fast and accurate numerical method need to be developed to analyzc the fibre modes in multilayer step-index fibres, as TFBG can excite hundreds of 
cladding modes in a SMF. When the fibre has a metal coating to increase the index sensitivity, we need to solve modes in the fibre with metal coatings, and this numerical method needs to solve fibre modes in multilayer step-index fibres with lossy and gain media (i.e. complex dielectric constants).

(ii) New formulations for calculating the hybrid mode coupling in mulatilayer step-index fibres with lossy and gain media are need for FBGs with and without a tiled angle. The specific transmission spectrum characteristics of TFBGs in SMF-28 fibre with a thin gold coating can then be simulated.

(iii) Novel sensors based on TFBGs in conventional SMFs need to be fully explored analytically and experimentally. The sensitivity characteristics of core and cladding modes in SMF with a TFBG to the mperature, strain and surrounding refractive index must be characterized to develup new types of optical sensors. The effect of cladding layer thickness and metal coating in SMFs must also be explored to improve core and cladding mode sensitivities. Another primary objective of the thesis is to further explore the sensing characteristics in the specific fibre photonic crystal fibre. A PCF is more expensive and the FBG is more difficult to write in the PCF, but the air-hole structure in PCFs could bring specific advantages that SMF don't have. So it is necessary to explore the sensitivity characteristics of core and cladding modes in PCF with a FBG to the ternperature, strain and surrounding refractive index. 


\section{Chapter 3}

\section{Optical Fibre Modes in Multilayer Step-Index Fibres}

\section{with Lossless, Lossy and Gain Media}

\subsection{Introduction}

Guided. leaky and radiation modes are fundamental concepts in optical fibre and waveguiding theory. Knowledge of mode propagation characteristics is essential to the design of numerous optical fibre devices, passive and active components such as fibre lasers, EDFA, optical fibre filters and couplers, and optical fibre sensors. Numerical methods that can efficiently and accurately model optical fibres are thus of obvious importance since they are used as a basic tool in the design process.

There are many kinds of optical fibres, most of them can be described as step-index fibres and graded index fibres. In the case of graded index fibre, it can be approximated by al scries of concentric layers of dielectric and treated as a multilayer step-index fibre. When fibres are used as telecommunication transmission media, they are mainly weakly guided, and their index contrast is small, so the scalar wave equation can be used to obtain the linearly-polarized modes (LP modes). However, when the index contrast becomes larger, the LP approximation becomes inaccurate, so a full vector analysis is necessary [35] and hybrid modes, transverse electric and magnetic modes need to be 
solved. In our experimental work, thinned SMF and the standard SMF-28 fibre with metal coating are widely used for biomedical sensing applications and for other sensor applications; in wher sensor applications, special fibres such as fibres with polymer layers also used for specific senser purpose such as the high thermal coefficient and sensitive to the humidity and gases. For those specific fibres, an accurate numerical tool is needed to analyze the fibre modes. There are several methods which can be used to solve the veciorial and scalar wave equations [15], but probably the best choice for a general, quick algorithm is the method developed by Yeh and Lindgren [16]. The method is similar to the transfer matrix method used for the analysis of planar waveguides [17]. Both methods use a matrix to express the fields at one side of a layer given their values at the other side, and the whole structure is then characterized by cascading the layers by matrix multiplication except in the method of Yeh and Lindgren, a set of inverse matrices need to be calculated. The numerical calculation of the inverse matrix sometimes will not be accurate especially when the matrix is close to singular. The inverse transfer matrix can be obtained analytically by decomposing the matrix to two simple matrices [18], whose inverse matrices can be obtained analytically. This provides a chance to develop an efficient and accurate fibre mode solver in lossy and gainy media by using the similar algorithm to that used in multilayer planar optical waveguides $[36,37]$.

In this Chapter, we propose a fast and accurate method to solve the dispersion equation for lossless, lossy and gain media. Compared with the literature published before, here the analytical inverse matrices are derived and a different algorithm to rapid 
solve the dispersion equations will be discussed. Some examples of different optical fibre structures are solved and compared with examples from literature and good agreement is obtained.

\subsection{Maxwell's Equations in Cylindrical Coordinates}

\subsubsection{General Wave Equations}

Maxwell's equations in their general form are written as [35]:

$$
\begin{aligned}
& \nabla \times \vec{E}=-\frac{\partial \vec{B}}{\partial t} \\
& \nabla \times \vec{H}=\frac{\partial \bar{D}}{\partial t}+\bar{J} \\
& \nabla \cdot \vec{D}=-\rho \\
& \nabla \cdot \vec{B}=0
\end{aligned}
$$

and the constitutive relations are:

$$
\begin{aligned}
& \vec{D}=\varepsilon \vec{E} \\
& \vec{B}=\mu \vec{H}
\end{aligned}
$$

We assume source free and time dependent harmonic fields with an $e^{\text {fex }}$ dependence, from Eqs (3.1) and (3.2), we obtain:

$$
\begin{aligned}
& \nabla \times \overrightarrow{\mathrm{E}}=j \omega \mu \overrightarrow{\mathrm{H}} \\
& \nabla \times \overrightarrow{\mathrm{H}}=-j \omega \varepsilon \overrightarrow{\mathrm{E}} \\
& \nabla \cdot \overrightarrow{\mathrm{E}}=0 \\
& \nabla \cdot \overline{\mathrm{H}}=0
\end{aligned}
$$


Taking the curl of Eq. (3.3a) and using Eq. (3.3b) yields:

$$
\nabla \times(\nabla \times \overrightarrow{\mathrm{E}})=-j \omega \mu \nabla \times \overrightarrow{\mathrm{H}}=\omega^{2} \mu \varepsilon \overrightarrow{\mathrm{E}}
$$

Using the vector identity $\nabla \times \nabla \times \bar{A}=\nabla(\nabla \cdot \bar{A})-\nabla^{2} \bar{A}$, and noting that $\nabla \cdot \overline{\mathrm{E}}=0$, yields:

$$
\nabla^{2} \overrightarrow{\mathrm{E}}+\omega^{2} \mu \varepsilon \overline{\mathrm{E}}=0
$$

Equation (3.5) is referred to as the wave equation or Helmholtz equation for $\bar{E}$. In the same manner, an identical equation for $\overline{\mathrm{H}}$ can be derived:

$$
\nabla^{2} \overrightarrow{\mathrm{H}}+\omega^{2} \mu \varepsilon \overrightarrow{\mathrm{H}}=0
$$

\subsubsection{General Solutions for HE/EH, TE, and TM Waves}

The electric and magnetic fields for arbitrary microwave transmission lines and optical waveguides that are invariant along $z$ and for propagation along the $+z$-axis, can be written as:

$$
\begin{aligned}
& \overline{\mathrm{E}}(r, \phi, z)=\left[\bar{e}_{t}(r, \phi)+\hat{z} e_{z}(r, \phi)\right] e^{-\jmath \beta z} \\
& \overline{\mathrm{H}}(r, \phi, z)=\left[\bar{h}_{t}(r, \phi)+\hat{z} h_{z}(r, \phi)\right] e^{-j \beta z}
\end{aligned}
$$

where $\vec{e}_{t}(r, \phi)$ and $\vec{h}_{t}(r, \phi)$ are the transverse electric and magnetic field components, $e_{z}(r, \phi)$ and $h_{z}(r, \phi)$ are the longitudinal electric and magnetic field components, and $\beta$ is the propagation constant. From Eqs. (3.3a) and (3.3b) with a $e^{-i \beta-} z$-dependence, six component equations can be derived:

$$
\frac{1}{r}\left(\frac{\partial E_{z}}{\partial \phi}+j r \beta E_{\phi}\right)=-j \omega \mu H_{r}
$$




$$
\begin{aligned}
& j \beta E_{\phi}+\frac{\partial E_{z}}{\partial r}=j \omega \mu H_{\phi} \\
& \frac{1}{r}\left[\frac{\partial}{\partial r}\left(r E_{\phi}\right)-\frac{\partial E_{r}}{\partial \phi}\right]=-j \omega \mu H_{z} \\
& \frac{1}{r}\left(\frac{\partial H_{z}}{\partial \phi}+j r \beta H_{\phi}\right)=j \omega \varepsilon E_{r} \\
& j \beta H_{r}+\frac{\partial H_{z}}{\partial r}=-j \omega \varepsilon E_{\phi} \\
& \frac{1}{r}\left[\frac{\partial}{\partial r}\left(r H_{\phi}\right)-\frac{\partial H_{r}}{\partial \phi}\right]=j \omega \varepsilon E_{z}
\end{aligned}
$$

Eqs. (3.8) can be solved for the four transverse field components in terms of $E_{z}$ and $H_{z}$ as follows:

$$
\begin{aligned}
& E_{r}=-\frac{j}{q^{2}}\left(\beta \frac{\partial E_{z}}{\partial r}+\frac{\omega \mu}{r} \frac{\partial H_{z}}{\partial \phi}\right) \\
& E_{\phi}=-\frac{j}{q^{2}}\left(\frac{\beta}{r} \frac{\partial E_{z}}{\partial \phi}-\omega \mu \frac{\partial H_{z}}{\partial r}\right) \\
& H_{r}=\frac{j}{q^{2}}\left(\frac{\omega \varepsilon}{r} \frac{\partial E_{z}}{\partial \phi}-\beta \frac{\partial H_{z}}{\partial r}\right) \\
& H_{\phi}=-\frac{j}{q^{2}}\left(\omega \varepsilon \frac{\partial E_{z}}{\partial r}+\frac{\beta}{r} \frac{\partial H_{z}}{\partial \phi}\right)
\end{aligned}
$$

where $q^{2}=\omega^{2} \varepsilon \mu-\beta^{2}$

Substituting the Eqs. (3.9c) and (3.9d) into Eq. (3.8f) results in the wave equation in cylindrical coordinates 


$$
\frac{\partial^{2} E_{z}}{\partial r^{2}}+\frac{1}{r} \frac{\partial E_{z}}{\partial r}+\frac{1}{r^{2}} \frac{\partial^{2} E_{z}}{\partial \phi^{2}}+q^{2} E_{z}=0
$$

and substituting the Eqs. (3.9a) and (3.9b) into Eq. (3.8c) we obtain

$$
\frac{\partial^{2} H_{z}}{\partial r^{2}}+\frac{1}{r} \frac{\partial H_{z}}{\partial r}+\frac{1}{r^{2}} \frac{\partial^{2} H_{z}}{\partial \phi^{2}}+q^{2} h_{z}=0
$$

Eqs. (3.10) and (3.11) each contain either only $E_{z}$ or only $H_{z}$. It seems that the two longitudinal components of $\vec{E}$ and $\vec{H}$ are uncoupled and can be chosen arbitrarily, but in general they are coupled by the bo'undary conditions of electromagnetic fields as hybrid modes. When the boundary conditions do not lead to coupling between the field components, such uncoupled mode solutions can be obtained as $E_{z}=0$ for transverse electric (TE) modes or $H_{z}=0$ for transverse magnetic (TM) modes.

\subsection{Wave Equations for Step-Index Fibres}

To solve the Eq. (3.10) for step-index optical fibres, the separation of the variables method can be used, which assumes the solution of $E_{z}$ can be written as

$$
E_{z}=A R(r) \Phi(\phi) Z(z)
$$

where

$$
Z(z)=e^{-\jmath \beta z}
$$

as the $z$ dependent in optical fibre, because the circular symmetry of the fibre, each field component will not change when the coordinate $\phi$ is increased by $2 \pi$, where the $v$ can be either po: $\quad$ r negative but must be an integer, thus mean we can write $\phi$ dependence as: 


$$
\Phi(\phi)=e^{\mu \phi}
$$

Now $E_{z}$ can be written as follows

$$
E_{z}=A R(r) e^{j r o} e^{-j \beta z}
$$

Substituling the Eq. (3.15) into Eq. (3.10), the wave equation for $E_{z}$ becomes:

$$
\frac{\partial^{2} R}{\partial r^{2}}+\frac{1}{r} \frac{\partial R}{\partial r}+\left(q^{2}-\frac{v^{2}}{r^{2}}\right) R=0
$$

Eq. (3.16) is a well-known differential equation for Bessel functions [38]. An exactly identical equation can be derived for $H_{z}$. In Sections 3.2 and 3.3 we have briefly reviewed wave equations in cylindrical coordinate, all the material in sections 3.2 an 3.3 can be found in textbooks such as $[35,39]$.

\subsection{Hybrid Modes in Multilayer Step-Index Optical Fibres}

\subsubsection{Dispersion Equation of Hybrid Modes}

The Fig. 3.1 shows the cross-section of an m layers step index fibre.

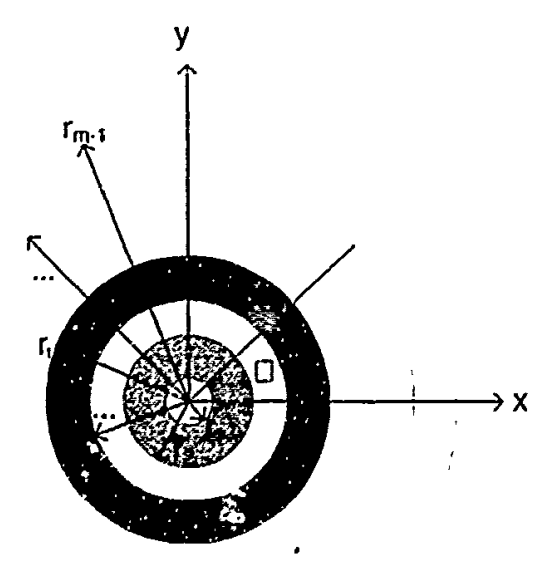

Figure 3.1: The cross-section of $m$ layer step-index fibre 
Solving for Maxwell's equations in cylindrical coordinates give the following fields for the layer $i\left[n_{e f f}<n_{i}\left(q^{2}>0\right)\right]: r_{i-1} \leq r \leq r_{i}[15]$

$$
\begin{aligned}
& e_{r}=\frac{\beta u_{i}}{\omega \varepsilon_{t}}\left[A_{t} J_{v}^{\prime}\left(u_{i} r\right)+B_{i} Y_{v}^{\prime}\left(u_{i} r\right)\right]-\frac{j v}{r}\left[C_{t} J_{v}\left(u_{t} r\right)+D_{t} Y_{v}\left(u_{i} r\right)\right] e^{j v \phi} \\
& e_{\phi}=-\frac{j \beta v}{\omega \varepsilon_{i} r^{r}}\left[A_{t} J_{v}\left(u_{t} r\right)+B_{t} Y_{v}\left(u_{i} r\right)\right]+u_{i}\left[C_{i} J_{v}^{\prime}\left(u_{t} r\right)+D_{t} Y_{v}^{\prime}\left(u_{i} r\right)\right] e^{j: \phi} \\
& e_{z}=\frac{u_{i}^{2}}{j \omega \varepsilon_{t}}\left[A_{t} J_{v}\left(u_{i} r\right)+B_{t} Y_{v}\left(u_{t} r\right)\right] e^{j v q} \\
& h_{r}=-\frac{j v}{r}\left[A_{i} J_{v}\left(u_{i} r\right)+B_{i} Y_{v}\left(u_{i} r\right)\right]-\frac{\beta u_{i}}{\omega \mu}\left[C_{i} J_{v}^{\prime}\left(u_{i} r\right)+D_{i} Y_{v}^{\cdot}\left(u_{i} r\right)\right] e^{j v \phi} \\
& h_{\delta}=-u_{i}\left[A_{i} J_{v}^{\prime}\left(u_{i} r\right)+B_{i} Y_{y}^{\prime}\left(u_{i} r\right)\right]-\frac{j \beta v}{\omega \mu r}\left[C_{i} J_{v}\left(u_{i} r\right)+D_{i} Y_{v}\left(u_{i} r\right)\right] e^{j v o} \\
& h_{z}=\frac{u_{i}^{2}}{j \omega \mu}\left[C_{i} J_{v}\left(u_{i} r\right)+D_{i} Y_{v}\left(u_{i} r\right)\right] e^{j v \phi}
\end{aligned}
$$

Where $u_{i}^{2}=k^{2} n_{t}^{2}-\beta^{2}, k=2 \pi / \lambda$ and $\lambda$ is the wavelength in vacuum, and $J_{v}, Y_{v}, J_{v}$ and $Y_{v}$ are the first kind and second kind Bessel functions and their derivatives in $v$ order. The difference in Eq. (3.17) here with Tsao's [15] is that we use $e^{j(c x-i z)}$ instead of $e^{j(\beta z-\alpha)}$. From Eq. (3.17) we can see that there are four unknown field coefficients: $A_{l}$, $B_{1}, C_{1}$ and $D_{1}$ in each layer $i$, therefore four field components are needed to form the transfer matrix. Because all field components have the same $e^{\text {jo }}$ term, this term is removed from following equations for simplicity and it won't affect the result of the effective index of the mode. 
$\left[\begin{array}{c}j \omega \varepsilon_{v} e_{z} \\ h_{\phi} \\ j \omega \mu h_{z} \\ e_{\phi}\end{array}\right]=\left[\begin{array}{cccc}u_{t}^{2} J_{v}\left(u_{t} r\right) / u_{t}^{2} & u_{t}^{2} Y_{v}\left(u_{t} r\right) / u_{t}^{2} & 0 & 0 \\ -u_{t}^{\prime} J_{v}^{\prime}\left(u_{t} r\right) & -u_{t} Y_{v}^{\prime}\left(u_{t} r\right) & -j \beta v J_{v}\left(u_{t} r\right) / \omega \mu r & -j \beta v Y_{v}\left(u u_{t} r\right) / \omega \mu r \\ 0 & 0 & u_{t}^{2} J_{v}\left(u_{t} r\right) & u_{t}^{2} Y_{v}\left(u_{t} r\right) \\ -j \beta v J_{v}\left(u_{t} r\right) / \omega \varepsilon_{v} n_{t}^{2} r & -j \beta v Y_{v}\left(u_{t} r\right) / \omega \varepsilon_{v} n_{t}^{2} r & u_{t} J_{v}^{\prime}\left(u_{t} r\right) & u_{t} Y_{v}^{\prime}\left(u_{t} r\right)\end{array}\right]\left[\begin{array}{c}A_{t} \\ B_{t} \\ C_{t} \\ D_{t}\end{array}\right]$

Substituting $j C_{1} \rightarrow C_{1}$ and $j D_{1} \rightarrow D_{1}$ in the eq. (3.18) can eliminate the complex number $j$ in the matrix and make the dispersion equations pure real for lossless step-index fibres to reduce the computer calculation time for the optical fibre modes and their fields.

$\left[\begin{array}{c}j \omega \varepsilon_{0} e_{z} \\ h_{0} \\ \omega \mu h_{z} \\ j e_{\phi}\end{array}\right]=\left[\begin{array}{cccc}u_{t}^{2} J_{v}\left(u_{t} r\right) / n_{t}^{2} & u_{t}^{2} Y_{v}\left(u_{t} r\right) / n_{t}^{2} & 0 & 0 \\ -u_{t} J_{v}^{\prime}\left(u_{t} r\right) & -u_{t} Y_{v}^{\prime}\left(u_{i} r\right) & \sigma_{1} J_{v}\left(u_{t} r\right) / r & \sigma_{1} Y_{v}\left(u_{i} r\right) / r \\ 0 & 0 & u_{t}^{2} J_{v}\left(u_{t} r\right) & u_{i}^{2} Y_{v}\left(u_{i} r\right) \\ \sigma_{2} J_{v}\left(u_{t} r\right) / n_{t}^{2} r & \sigma_{2} Y_{v}\left(u_{t} r\right) / n_{t}^{2} r & -u_{t} J_{v}^{\prime}\left(u_{t} r\right) & -u_{i} Y_{v}^{\prime}\left(u_{i} r\right)\end{array}\right]\left[\begin{array}{c}A_{t} \\ B_{t} \\ C_{t} \\ D_{t}\end{array}\right]=M_{t}\left[\begin{array}{c}A_{i} \\ B_{1} \\ C_{t} \\ D_{1}\end{array}\right]$

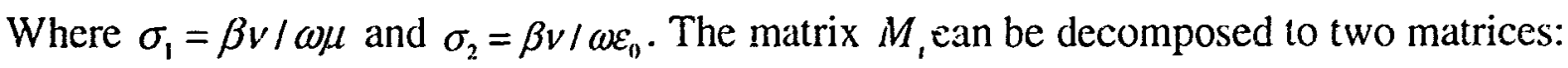

one sparse matrix $M_{i l}$ and one block matrix $M_{i l l}$.

$M_{t}=\left[\begin{array}{cccc}1 / n_{t}^{2} & 0 & 0 & 0 \\ 0 & -1 & \sigma_{1} / u_{t}^{2} r & 0 \\ 0 & 0 & 1 & 0 \\ \sigma_{2} / u_{t}^{2} n_{t}^{2} r & 0 & 0 & -1\end{array}\right]\left[\begin{array}{cccc}u_{t}^{2} J_{v}\left(u_{t} r\right) & u_{t}^{2} Y_{v}\left(u_{t} r\right) & 0 & 0 \\ u_{t} J_{v}^{\prime}\left(u_{t} r\right) & u_{t} Y_{v}^{\prime}\left(u_{t} r\right) & 0 & 0 \\ 0 & 0 & u_{t}^{2} J_{v}\left(u_{t} r\right) & u_{t}^{2} Y_{v}\left(u_{t} r\right) \\ 0 & 0 & u_{t} J_{v}^{\prime}\left(u_{t} r\right) & u_{t} Y_{v}^{\prime}\left(u_{t} r\right)\end{array}\right]=M_{t \prime} M_{t \prime}$

The inverse matrix of $M_{i}$ can be obtained analytically as following

$$
M_{\imath}^{-1}=M_{t l l}^{-1} M_{t}^{-1}
$$

where

$$
M_{\prime \prime}^{-1}=\left[\begin{array}{cccc}
n_{1}^{2} & 0 & 0 & 0 \\
0 & -1 & \sigma_{1} / u_{1}^{2} r & 0 \\
0 & 0 & 1 & 0 \\
\sigma_{2} / u_{t}^{2} r & 0 & 0 & -1
\end{array}\right]
$$

and 


$$
M_{i l l}^{-1}=\frac{1}{u_{t}^{3}\left[J_{v}\left(u_{t} r\right) Y_{v}^{\prime}\left(u_{t} r\right)-Y_{v}\left(u_{t} r\right) J_{v}^{\prime}\left(u_{t} r\right)\right]}\left[\begin{array}{cccc}
u_{t} Y_{v}^{\prime}\left(u_{t} r\right) & -u_{t}^{2} Y_{v}\left(u_{t} r\right) & 0 & 0 \\
-u_{t} J_{v}^{\prime}\left(u_{t} r\right) & u_{t}^{2} J_{v}\left(u_{t} r\right) & 0 & 0 \\
0 & 0 & u_{t} Y_{v}^{\prime}\left(u_{t} r\right) & -u_{1}^{2} Y_{v}(u, r) \\
0 & 0 & -u_{t} J_{v}^{\prime}\left(u_{t} r\right) & u_{1}^{2} J_{v}\left(u_{t} r\right)
\end{array}\right]
$$

Noting that $J_{v}(x) Y_{v}^{\prime}(x)-Y_{v}(x) J_{v}^{\prime}(x)=2 / \pi x$ [38], so we obtain

$$
M_{t l}^{-1}=\frac{\pi r}{2}\left[\begin{array}{cccc}
Y_{v}^{\prime}\left(u_{i} r\right) / u_{t} & -Y_{v}\left(u_{i} r\right) & 0 & 0 \\
-J_{v}^{\prime}\left(u_{t} r\right) / u_{t} & J_{v}\left(u_{t} r\right) & 0 & 0 \\
0 & 0 & Y_{v}^{\prime}\left(u_{t} r\right) / u_{t} & -Y_{v}\left(u_{t} r\right) \\
0 & 0 & -J_{v}^{\prime}\left(u_{i} r\right) / u_{i} & J_{v}\left(u_{t} r\right)
\end{array}\right]
$$

For $n_{\text {eff }}>n_{t}\left(q^{2}<0\right)$, the matrix $M$, and its inverse matrices can be obtained as follows:

$$
\begin{aligned}
& M_{1}=\left[\begin{array}{cccc}
-w_{i}^{2} I_{v}\left(w_{i} r\right) / n_{i}^{2} & -w_{i}^{2} K_{v}\left(w_{i} r\right) / n_{i}^{2} & 0 & 0 \\
-w_{i} I_{v}^{\prime}\left(w_{i} r\right) & -w_{t} K_{v}^{\prime}\left(w_{t} r\right) & \sigma_{1} I_{v}\left(w_{i} r\right) / r & \sigma_{1} K_{v}\left(w_{i} r\right) / r \\
0 & 0 & -w_{i}^{2} I_{v}\left(w_{t} r\right) & -w_{t}^{2} K_{v}\left(w_{i} r\right) \\
\sigma_{2} I_{v}\left(w_{t} r\right) / n_{i}^{2} r & \sigma_{2} K_{v}\left(w_{i} r\right) / n_{i}^{2} r & -w_{i} I_{v}^{\prime}\left(w_{i} r\right) & -w_{i} K_{v}\left(w_{t} r\right)
\end{array}\right] \\
& M_{t l}=\left[\begin{array}{cccc}
-1 / n_{i}^{2} & 0 & 0 & 0 \\
0 & -1 & \sigma_{1} / w_{i}^{2} r & 0 \\
0 & 0 & -1 & 0 \\
\sigma_{2} / w_{i}^{2} l_{i}^{2} r & 0 & 0 & -1
\end{array}\right] \\
& M_{i l l}=\left[\begin{array}{cccc}
w_{i}^{2} I_{v}\left(w_{i} r\right) & w_{1}^{2} K_{v}\left(w_{t} r\right) & 0 & 0 \\
w_{i} I_{v}^{\prime}\left(w_{i} r\right) & w_{i} K_{v}^{\prime}\left(w_{i} r\right) & 0 & 0 \\
0 & 0 & w_{1}^{2} I_{v}\left(w_{t} r\right) & w_{1}^{2} K_{v}\left(w_{i} r\right) \\
0 & 0 & w_{i} I_{v}^{\prime}\left(w_{l} r\right) & w_{t} K_{v}^{\prime}\left(w_{t} r\right)
\end{array}\right] \\
& M_{l}^{-1}=\left[\begin{array}{cccc}
-n_{i}^{2} & 0 & 0 & 0 \\
0 & -1 & -\sigma_{1} / w_{1}^{2} r & 0 \\
0 & 0 & -1 & 0 \\
-\sigma_{2} / w_{1}^{2} r & 0 & 0 & -1
\end{array}\right]
\end{aligned}
$$

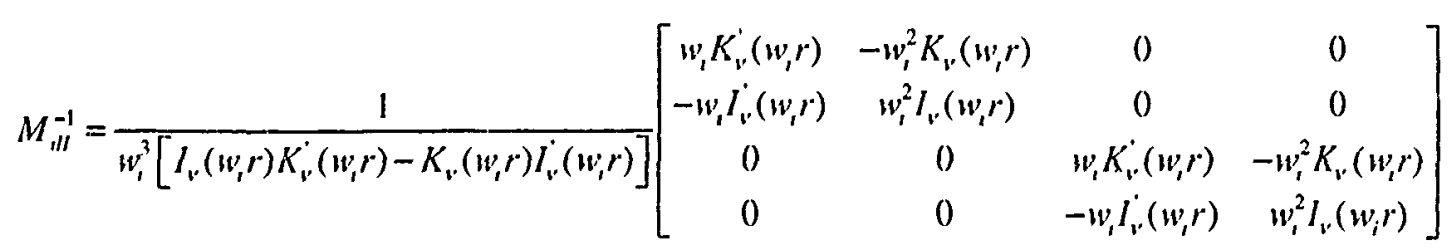


Where $I_{v}, K_{v}, I_{v}$ and $K_{v}^{\prime}$ are the modified first kind and modified second kind Bessel functions and their derivatives in $v$ orter. At the boundary $r=r_{t}$, the tangential fields $e_{z}$, $e_{\phi}, h_{z}$ and $h_{\phi}$ must be continuous to give:

$$
M_{i+1}\left(r_{i}\right)\left[\begin{array}{c}
A_{i+1} \\
B_{i+1} \\
C_{t+1} \\
D_{t+1}
\end{array}\right]=M_{i}\left(r_{i}\right)\left[\begin{array}{l}
A_{t} \\
B_{i} \\
C_{i} \\
D_{i}
\end{array}\right]
$$

Multiply by $M_{i+1}^{-1}\left(r_{i}\right)$ on both sides of Eq. (3.30) to obtain:

$$
\left[\begin{array}{c}
A_{t+1} \\
B_{t+1} \\
C_{i+1} \\
D_{i+1}
\end{array}\right]=M_{i+1}^{-1}\left(r_{i}\right) M_{i}\left(r_{i}\right)\left[\begin{array}{l}
A_{t} \\
B_{i} \\
C_{t} \\
D_{t}
\end{array}\right]
$$

Considering all the layer boundaries, the relations of the tangential fields at the first layer and last layer can be written as the following (similar to the transfer matrix method):

$$
M_{m}\left(r_{m-1}\right)\left[\begin{array}{l}
A_{m} \\
B_{m} \\
C_{m} \\
D_{m}
\end{array}\right]=M_{m-1}\left(r_{m-1}\right) M_{m-1}^{-1}\left(r_{m-2}\right) M_{m-2}\left(r_{m-2}\right) \cdots M_{2}\left(r_{2}\right) M_{2}^{-1}\left(r_{1}\right) M_{1}\left(r_{i}\right)\left[\begin{array}{l}
A_{1} \\
B_{1} \\
C_{1} \\
D_{1}
\end{array}\right]
$$

and multiplying again by $M_{m l}^{-1}\left(r_{m-1}\right)$ on both side of Eq. (3.32), we obtain:

$$
M_{m l l}\left(r_{m-1}\right)\left[\begin{array}{l}
A_{m} \\
B_{m} \\
C_{m} \\
D_{m}
\end{array}\right]=M_{m !}^{-1}\left(r_{m-1}\right) M_{m-1}\left(r_{m-1}\right) M_{m-1}^{-1}\left(r_{m-2}\right) M_{m-2}\left(r_{m-2}\right) \cdots M_{2}\left(r_{2}\right) M_{2}^{-1}\left(r_{1}\right) M_{1}\left(r_{1}\right)\left[\begin{array}{l}
A_{1} \\
B_{1} \\
C_{1} \\
D_{1}
\end{array}\right]
$$

For the fibre whose first layer has the refractive index larger than effective index, fields can not be infinite at the centre ci the fibre, so $B_{1}$ and $D_{1}$ need to be zero. Furthermore 
fields must converge at the last layer, so the field coefficients $A_{m}$ and $C_{m}$ must be zero for guided modes. These boundary conditions simplify the matrices $M_{1}$ and $M_{m l l}$ :

$$
\begin{gathered}
M_{1}=\left[\begin{array}{cccc}
u_{1}^{2} J_{v}\left(u_{1} r_{1}\right) / n_{1}^{2} & 0 & 0 & 0 \\
-u_{1} J_{v}^{\prime}\left(u_{1} r_{1}\right) & 0 & \sigma_{1} J_{v}\left(u_{1} r_{1}\right) / r_{1} & 0 \\
0 & 0 & u_{1}^{2} J_{v}\left(u_{1} r_{1}\right) & 0 \\
\sigma_{2} J_{v}\left(u_{1} r_{1}\right) / n_{1}^{2} r_{1} & 0 & -u_{1} J_{v}^{\prime}\left(u_{1} r_{1}\right) & 0
\end{array}\right] \\
M_{m l l}=\left[\begin{array}{cccc}
0 & w_{m}^{2} K_{v}\left(w_{m} r_{m-1}\right) & 0 & 0 \\
0 & w_{m} K_{v}^{\prime}\left(w_{m} r_{m-1}\right) & 0 & 0 \\
0 & 0 & 0 & w_{m}^{2} K_{v}\left(w_{m} r_{m-1}\right) \\
0 & 0 & 0 & w_{m} K_{v}^{\prime}\left(w_{m} r_{m-1}\right)
\end{array}\right]
\end{gathered}
$$

The Eq. (3.33) can now simplify as:

$$
\left[\begin{array}{cccc}
0 & w_{m}^{2} K_{v}\left(w_{m} r_{m-1}\right) & 0 & 0 \\
0 & w_{m} K_{v}^{\prime}\left(w_{m} r_{m-1}\right) & 0 & 0 \\
0 & 0 & 0 & w_{m}^{2} K_{v}\left(w_{m} r_{m-1}\right) \\
0 & 0 & 0 & w_{m} K_{v}^{\prime}\left(w_{m} r_{m-1}\right)
\end{array}\right]\left[\begin{array}{l}
0 \\
B_{m} \\
0 \\
D_{m}
\end{array}\right]=\left[\begin{array}{cccc}
M_{11} & 0 & M_{13} & 0 \\
M_{21} & 0 & M_{23} & 0 \\
M_{31} & 0 & M_{33} & 0 \\
M_{41} & 0 & M_{43} & 0
\end{array}\right]\left[\begin{array}{l}
A_{1} \\
0 \\
C_{1} \\
0
\end{array}\right]
$$

where $M_{l l}, M_{2 l}, \cdots$, and $M_{43}$ are the elements of matrix $M$,

$$
M=M_{m l}^{-1}\left(r_{m-1}\right) M_{m-1}\left(r_{m-1}\right) M_{m-1}^{-1}\left(r_{m-2}\right) M_{m-2}\left(r_{m-2}\right) \cdots M_{2}\left(r_{2}\right) M_{2}^{-1}\left(r_{1}\right) M_{1}\left(r_{1}\right)
$$

Reorganizing the Eq. (3.36), we obtain

$$
\left[\begin{array}{cccc}
M_{11} & M_{13} & w_{m}^{2} K_{v}\left(w_{m} r_{m-1}\right) & 0 \\
M_{21} & M_{23} & w_{m} K_{v}^{\prime}\left(w_{m} r_{m-1}\right) & 0 \\
M_{31} & M_{3 ;} & 0 & w_{m}^{2} K_{v}\left(w_{m} r_{m-1}\right) \\
M_{41} & M_{43} & 0 & w_{m} K_{v}^{\prime}\left(w_{m} r_{m-1}\right)
\end{array}\right]\left[\begin{array}{l}
A_{1} \\
C_{1} \\
-B_{m} \\
-D_{m}
\end{array}\right]=0
$$

Because coefficients $A_{1}, C_{1}, B_{m}$ and $D_{m}$ can not be zero for the non-trivial solution of the Eq. (3.38), then we have 


$$
\operatorname{Det}\left[\begin{array}{cccc}
M_{11} & M_{13} & w_{m}^{2} K_{v}\left(w_{m} r_{m-1}\right) & 0 \\
M_{21} & M_{23} & w_{m} K_{v}^{\prime}\left(w_{m} r_{m-1}\right) & 0 \\
M_{31} & M_{33} & 0 & w_{m}^{2} K_{v}\left(w_{m} r_{m-1}\right) \\
M_{41} & M_{43} & 0 & w_{m} K_{v}^{\prime}\left(w_{m} r_{m-1}\right)
\end{array}\right]=0
$$

Eq. (3.39) is the dispersion equations. The roots of this dispersion equation are the propagation constant or effective index of the hybrid modes. The dispersion equation $F\left(\beta_{i}\right)$ or $F\left(n_{e f f}^{i}\right)$ for $\mathrm{HE} / \mathrm{EH}$ hybrid modes can take the following form:

$$
F=a x^{2}+b x+c=0
$$

Where $\beta_{i}$ is the propagation constant and $n_{e f f}^{i}$ is the effective index of mode $i$,

$$
\begin{aligned}
& a=M_{13} M_{31}-M_{11} M_{33} \\
& b=M_{11} M_{43}-M_{13} M_{41}+M_{21} M_{33}-M_{23} M_{31} \\
& c=M_{23} M_{41}-M_{21} M_{43}
\end{aligned}
$$

and

$$
x=K_{v}^{\prime}\left(w_{m} r_{m-1}\right) / w_{m} K_{v}\left(w_{m} r_{m-1}\right)
$$

Solving the dispersion equation gives the effective indices, which are the roots of the dispersion equation, for different modes. After obtaining the effective index of each mode (or the propagation constant), the field distributions can be calculated.

\subsubsection{The Fields of Hybrid Modes}

By using the Bessel function relationships to the transverse fields: the fields in each layer can be written as follows:

The fields for the first layer 1 : 


$$
\begin{aligned}
& \left.e_{r}=\left[-\frac{\beta u_{1}}{2 \omega \varepsilon_{1}} A_{1}\left(J_{v-1}\left(u_{1} r\right)-J_{v+1}\left(u_{1} r\right)\right)+\frac{u_{1}}{2} C_{1}\left(J_{v-1}\left(u_{1} r\right)+J_{v+1}\left(u_{1} r\right)\right)\right] \operatorname{ex}\right)(j v \phi) \\
& e_{\phi}=j\left[-\frac{\beta u_{1}}{2 \omega \varepsilon_{1}} A_{1}\left(J_{v-1}\left(u_{1} r\right)+J_{v+1}\left(u_{1} r\right)\right)+\frac{u_{1}}{2} C_{1}\left(J_{v-1}\left(u_{1} r\right)-J_{v+1}\left(u_{1} r\right)\right)\right] \exp (j v \phi) \\
& e_{z}=-j \frac{u_{1}^{2}}{\omega \varepsilon_{1}} A_{1} J_{v^{\prime}}\left(u_{1} r\right) \exp (j v \phi) \\
& h_{r}=j\left[\frac{u_{1}}{2} A_{1}\left(J_{v-1}\left(u_{1} r\right)+J_{v+1}\left(u_{1} r\right)\right)-\frac{\beta u_{1}}{2 \omega \mu} C_{1}\left(J_{v-1}\left(u_{1} r\right)-J_{v+1}\left(u_{1} r\right)\right)\right] \exp (j v \phi) \\
& h_{\phi}=\left[-\frac{u_{1}}{2} A_{1}\left(J_{v-1}\left(u_{1} r\right)-J_{v+1}\left(u_{1} r\right)\right)+\frac{\beta u_{1}}{2 \omega \mu} C_{1}\left(J_{v-1}\left(u_{1} r\right)+J_{v+1}\left(u_{1} r\right)\right)\right] \exp (j v \phi) \\
& h_{z}=\frac{u_{1}^{2}}{\omega \mu} C_{1} J_{v}\left(u_{1} r\right) \exp (j v \phi)
\end{aligned}
$$

The fields for layer i: $r_{i-1} \leq r \leq r_{t}$

if $n_{e f f}<n_{t}$,

$$
\begin{aligned}
& e_{r}=\left\{-\frac{\beta u_{t}}{2 \omega \varepsilon_{t}}\left[A_{t}\left(J_{v-1}\left(u_{t} r\right)-J_{v+1}\left(u_{t} r\right)\right)+B_{1}\left(Y_{v-1}(u, r)-Y_{v+1}\left(u_{t} r\right)\right)\right]\right. \\
& \left.+\frac{u_{t}}{2}\left[C_{1}\left(J_{v^{\prime}-1}\left(u_{t} r\right)+J_{v^{r+1}}\left(u_{t} r\right)\right)+D_{1}\left(Y_{r^{r}-1}\left(u_{t} r\right)+Y_{v^{\prime}+1}\left(u_{t} r\right)\right)\right]\right\} \exp (j \nu \phi) \\
& \varepsilon_{\phi}=j\left\{-\frac{\beta u_{i}}{2 \omega \varepsilon_{i}}\left[A_{\imath}\left(J_{v^{\prime}-1}\left(u_{\imath} r\right)+J_{v+1}\left(u_{\imath} r\right)\right)+B_{\imath}\left(Y_{v^{\prime}-1}\left(u_{\imath} r\right)+Y_{v+1}\left(u_{1} r\right)\right)\right]\right. \\
& \left.+\frac{u_{1}}{2}\left[C_{1}\left(J_{v-1}\left(u_{t} r\right)-J_{v^{\prime}+1}\left(u_{t} r\right)\right)+D_{1}\left(Y_{v^{\prime-1}}\left(u_{t} r\right)-Y_{v^{\prime}+1}\left(u_{i} r\right)\right)\right]\right\} \exp (j v \phi) \\
& e_{z}=-j \frac{u_{l}^{2}}{\omega \varepsilon_{l}}\left[A_{i} J_{v}\left(u_{i} r\right)+B_{l} Y_{v}\left(u_{t} r\right)\right] \exp (j v \phi)
\end{aligned}
$$




$$
\begin{aligned}
h_{r}= & j\left\{\frac{u_{t}}{2}\left[A_{t}\left(J_{v^{\prime}-1}\left(u_{t} r\right)+J_{v+1}\left(u_{t} r\right)\right)+B_{t}\left(Y_{v-1}\left(u_{t} r\right)+Y_{v+1}\left(u_{t} r\right)\right)\right]\right. \\
& \left.-\frac{\beta u_{t}}{2 \omega \mu}\left[C_{t}\left(J_{v-1}\left(u_{t} r\right)-J_{v+1}\left(u_{t} r\right)\right)+D_{t}\left(Y_{v-1}\left(u_{t} r\right)-Y_{v+1}\left(u_{t} r\right)\right)\right]\right\} \exp (j v \phi) \\
h_{\phi}= & \left\{-\frac{u_{t}}{2}\left[A_{t}\left(J_{v-1}\left(u_{t} r\right)-J_{v+t}\left({ }^{\prime} r\right)\right)+B_{t}\left(Y_{v-1}\left(u_{t} r\right)-Y_{v+1}\left(u_{t} r\right)\right)\right]\right. \\
& \left.+\frac{\beta u_{t}}{2 \omega \mu}\left[C_{t}\left(J_{v-1}\left(u_{t} r\right)+J_{v+1}\left(u_{t} r\right)\right)+D_{t}\left(Y_{v-1}\left(u_{t} r\right)+Y_{v+1}\left(u_{t} r\right)\right)\right]\right\} \exp (j v \phi) \\
h_{z}= & \frac{u_{t}^{2}}{\omega, \mu}\left[C_{t} J_{v}\left(u_{t} r\right)+D_{t} Y_{v}\left(u_{t} r\right)\right] \exp (j v \phi)
\end{aligned}
$$

if $n_{\text {eff }}>n_{i}$,

$$
\begin{aligned}
& e_{r}=\left\{-\frac{\beta w_{t}}{2 \omega \varepsilon_{t}}\left[A_{t}\left(I_{v-1}\left(w_{t} r\right)+I_{v+1}\left(w_{t} r\right)\right)-B_{t}\left(\Lambda_{v-1}^{r}\left(w_{t} r\right)-K_{v+1}\left(w_{t} r\right)\right)\right]\right. \\
& \left.+\frac{w_{i}}{2}\left[C_{l}\left(I_{v-1}\left(w_{\imath} r\right)-I_{v+1}\left(w_{t} r\right)\right)-D_{i}\left(K_{v^{\prime}-1}\left(w_{\imath} r\right)+K_{v+1}\left(w_{\imath} r\right)\right)\right]\right\} \exp (j v \phi) \\
& e_{o}=j\left\{-\frac{\beta w_{t}}{2 \omega \varepsilon_{1}}\left[A_{i}\left(I_{v-1}\left(w_{1} r\right)-I_{v+1}\left(w_{t} r\right)\right)-B_{1}\left(K_{v-1}\left(w_{1} r\right)+K_{v+1}\left(w_{1} r\right)\right)\right]\right. \\
& \left.+\frac{w_{t}}{2}\left[C_{t}\left(I_{v-1}\left(w_{t} r\right)+I_{v+1}\left(w_{t} r\right)\right)-D_{t}\left(K_{v-1}\left(w_{t} r\right)-K_{v+1}\left(w_{\imath} r\right)\right)\right]\right\} \exp (j v \phi) \\
& c_{:}=j \frac{w_{t}^{2}}{\omega \varepsilon_{t}}\left[A_{1} I_{v}\left(w_{t} r\right)+B_{t} K_{v}\left(w_{t} r\right)\right] \exp (j v \phi) \\
& h_{r}=j\left\{\frac{w_{i}}{2}\left[A_{1}\left(: \quad w_{t} r\right)-l_{v+1}\left(w_{t} r\right)\right)-B_{t}\left(K_{r-1}\left(w_{t} r\right)+K_{r+1}\left(w_{t} r\right)\right)\right] \\
& \left.-\frac{\beta w_{1}}{2 \omega \mu}\left[C_{1}\left(I_{v-1}\left(w_{\imath} r\right)+I_{v+1}\left(w_{1} r\right)\right)-D_{1}\left(K_{v-1}\left(w_{\imath} r\right)-K_{v+1}\left(w_{\imath} r\right)\right)\right]\right\} \exp (j v \phi)
\end{aligned}
$$




$$
\begin{aligned}
h_{\varphi}= & \left\{-\frac{w_{t}}{2}\left[A_{i}\left(I_{v-1}\left(w_{t} r\right)+I_{r+1}\left(w_{t} r\right)\right)-B_{i}\left(K_{r-1}\left(w_{t} r\right)-K_{r+1}\left(w_{i} r\right)\right)\right]\right. \\
& \left.+\frac{\beta w_{i}}{2 \omega \mu}\left[C_{i}\left(I_{v-1}\left(w_{i} r\right)-I_{v+1}\left(w_{i} r\right)\right)-D_{i}\left(K_{r-1}\left(w_{i} r\right)+K_{v+1}\left(w_{t} r\right)\right)\right]\right\} \exp (j v \phi) \\
h_{z}= & -\frac{w_{t}^{2}}{\omega \mu l}\left[C_{t} I_{v}\left(w_{i} r\right)+D_{i} K_{v}\left(w_{i} r\right)\right] \exp (j v \phi)
\end{aligned}
$$

the fields for last layer $m: r \geq r_{m}$

$$
\begin{aligned}
& e_{r}=\left[\frac{\beta w_{m}}{2 \omega \varepsilon_{m}} B_{m}\left(K_{v-1}\left(w_{m} r\right)-K_{v+1}\left(w_{m} r\right)\right)-\frac{w_{m}}{2} D_{m}\left(K_{v-1}\left(w_{m} r\right)+K_{v+1}\left(w_{m} r\right)\right)\right] \exp (j v \phi) \\
& e_{\phi}=j\left[\frac{\beta w_{m}}{2 \omega \varepsilon_{m}} B_{m}\left(K_{v-1}\left(w_{m} r\right)+K_{v+1}\left(w_{m} r\right)\right)-\frac{w_{m}}{2} D_{m}\left(K_{v-1}\left(w_{m} r\right)-K_{v+1}\left(w_{m} r\right)\right)\right] \exp (j v \phi) \\
& e_{z}=j \frac{w_{m}^{2}}{\omega \varepsilon_{m}} B_{m} K_{v}\left(w_{m} r\right) \exp (j v \phi) \\
& h_{r}=j\left[-\frac{w_{m}}{2} B_{m}\left(K_{v-1}\left(w_{m} r\right)+K_{v+1}\left(w_{m} r\right)\right)+\frac{\beta w_{m}}{2 \omega \mu} D_{m}\left(K_{v-1}\left(w_{m} r\right)-K_{v+1}\left(w_{m} r\right)\right)\right] \exp (j v \phi) \\
& h_{\varphi}=\left[\frac{w_{m}}{2} B_{m}\left(K_{v-1}\left(w_{m} r\right)-K_{v+1}\left(w_{m} r\right)\right)-\frac{\beta w_{m}}{2 \omega \mu} D_{m}\left(K_{v-1}\left(w_{m} r\right)+K_{v+1}\left(w_{m} r\right)\right)\right] \exp (j v \phi) \\
& h_{z}=-\frac{w_{m}^{2}}{\omega \mu} D_{m} K_{v}\left(w_{m} r\right) \exp (j v \phi)
\end{aligned}
$$

\subsection{Transverse Modes in Multilayer Step-Index Optical Fibres}

As discussed in section 3.3.2, there are analytical solutions of the Maxwell Equations for transverse electric and magnetic modes in step-index fibres. The characteristics of the transverse modes means that we only need solve either $H_{z}$ for TE modes $\left(E_{z}=0\right)$ and $E_{z}$ for $\mathrm{TM} \operatorname{modes}\left(H_{z}=C_{\text {, }}\right.$ 


\subsubsection{TE Modes}

For TE modes $e_{z}=0$, that gives $A_{i}=B_{i}=0$, from Eq. (3.19), we obtain

$$
\left[\begin{array}{c}
\omega \mu h_{z} \\
-j e_{\phi}
\end{array}\right]=\left[\begin{array}{ll}
u_{t}^{2} J_{v}\left(u_{i} r\right) & u_{i}^{2} Y_{v}\left(u_{t} r\right) \\
u_{t} J_{v}^{\prime}\left(u_{i} r\right) & u_{i} Y_{v}^{\prime}\left(u_{i} r\right)
\end{array}\right]\left[\begin{array}{l}
C_{t} \\
D_{i}
\end{array}\right]=M_{i}\left[\begin{array}{l}
C_{i} \\
D_{t}
\end{array}\right]
$$

the inverse matrix of $M_{1}$

$$
M_{t}^{-1}=\frac{\pi r}{2 u_{1}^{2}}\left[\begin{array}{cc}
u_{t} Y_{v}^{\prime}\left(u_{t} r\right) & -u_{t}^{2} Y_{v}\left(u_{t} r\right) \\
-u_{t} J_{v}^{\prime}\left(u_{r} r\right) & u_{t}^{2} J_{v}\left(u_{t} r\right)
\end{array}\right]
$$

For $n_{e f f}>n_{1}$,

$$
M_{i}=\left[\begin{array}{cc}
-w_{i}^{2} I_{v}\left(w_{r} r\right) & -w_{i}^{2} K_{v}\left(w_{i} r\right) \\
w_{i} I_{v}\left(w_{i} r\right) & w_{i} K_{v}^{\prime}\left(w_{i} r\right)
\end{array}\right]
$$

and the inverse matrix of $M_{i}$

$$
M_{i}^{-1}=\frac{1}{w_{i}^{3}\left(I_{v}^{\prime}\left(w_{t} r\right) K_{v}\left(w_{i} r\right)-I_{v}\left(w_{t} r\right) K_{v}^{\prime}\left(w_{t} r\right)\right)}\left[\begin{array}{cc}
w_{i} K_{v}^{\prime}\left(w_{t} r\right) & w_{i}^{2} K_{v}\left(w_{t} r\right) \\
-w_{i} l_{v}^{\prime}\left(w_{t} r\right) & -w_{i}^{2} I_{v}\left(w_{t} r\right)
\end{array}\right]
$$

applying the boundary conditions for each layer, we obtain

$$
M_{1}\left(r_{1}\right)\left[\begin{array}{c}
C_{1} \\
0
\end{array}\right]=M_{2}\left(r_{1}\right) M_{2}^{-1}\left(r_{2}\right) M_{3}\left(r_{2}\right) M_{3}^{-1}\left(r_{3}\right) \cdots M_{m-1}^{-1}\left(r_{m-1}\right) M_{m}\left(r_{m-1}\right)\left[\begin{array}{c}
0 \\
D_{m}
\end{array}\right]=M\left[\begin{array}{c}
0 \\
D_{m}
\end{array}\right]
$$

Reorganizing the Eq. (3.51), we have

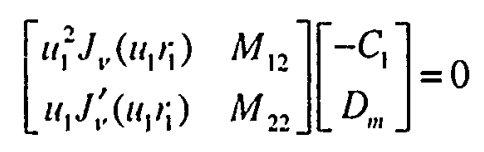

The dispersion equation of TE modes can be written as

$$
u_{1} J_{v}\left(u_{1} r_{1}\right) M_{22}-J_{v}^{\prime}\left(u_{1} r_{i}\right) M_{12}=0
$$


After solving the dispersion equation of TE modes, the effective index of each mode can be obtained. The field distributions of the mode can be calculated from Eqs. (3.43-3.46). by applying the field coefficients $A_{t}=B_{1}=0$.

\subsubsection{TM Modes}

For TM modes $h_{i}=0$, that gives $C_{i}=D_{t}=0$, from Eq. (3.19), we obtain

$$
\left[\begin{array}{c}
j \omega \varepsilon_{t} e_{i} \\
-h_{\phi}
\end{array}\right]=\left[\begin{array}{cc}
u_{t}^{2} J_{v}\left(u_{t} r\right) / n_{t}^{2} & u_{t}^{2} Y_{v}\left(u_{t} r\right) / n_{t}^{2} \\
u_{t} J_{v}^{\prime}\left(u_{t} r\right) & u_{t} Y_{v}^{\prime}\left(u_{t} r\right)
\end{array}\right]\left[\begin{array}{c}
A_{t} \\
B_{t}
\end{array}\right]=M_{t}\left[\begin{array}{l}
A_{t} \\
B_{t}
\end{array}\right]
$$

the inverse matrix of $M_{t}$

$$
M_{t}^{-1}=\frac{\pi n_{i}^{2} r}{2 u_{t}^{2}}\left[\begin{array}{ll}
u_{t} Y_{v}^{\prime}\left(u_{t} r\right) & \frac{-u_{t}^{2}}{n_{t}^{2}} Y_{v}\left(u_{t} r\right) \\
-u_{t} J_{v}^{\prime}\left(u_{t} r\right) & \frac{u_{t}^{2}}{n_{t}^{2}} J_{v}\left(u_{t} r\right)
\end{array}\right]
$$

For $n_{c f f}>n_{1}$,

$$
M_{1}=\left[\begin{array}{cc}
-w_{l}^{2} I_{v}\left(w_{1} r\right) / h_{t}^{2} & -w_{1}^{2} K_{v}\left(w_{t} r\right) / n_{t}^{2} \\
w_{1} I_{v}^{\prime}\left(w_{t} r\right) & w_{t} K_{v}^{\prime}\left(w_{t} r\right)
\end{array}\right]
$$

the inverse matrix of $M_{i}$

$$
M_{\cdot}^{-1}=\frac{n_{t}^{2}}{w_{i}^{3}\left(I_{v}^{\prime}\left(w_{i} r\right) K_{v}\left(w_{i} r\right)-I_{v}\left(w_{t} r\right) K_{v}^{\prime}\left(w_{i} r\right)\right)}\left[\begin{array}{cc}
w_{i} K_{v}^{\prime}\left(w_{t} r\right) & w_{i}^{2} K_{v}\left(w_{i} r\right) / n_{t}^{2} \\
-w_{t} I_{v}^{\prime}\left(w_{t} r\right) & -w_{t}^{2} I_{v}\left(w_{t} r\right) / n_{t}^{2}
\end{array}\right]
$$

applying the boundary conditions for each layer, we obtain

$$
M_{1}\left(r_{1}\right)\left[\begin{array}{c}
A_{1} \\
0
\end{array}\right]=M_{2}\left(r_{1}\right) M_{2}^{-1}\left(r_{2}\right) M_{3}\left(r_{2}\right) M_{3}^{-1}\left(r_{3}\right) \cdots M_{m-1}^{-1}\left(r_{m-1}\right) M_{m}\left(r_{m-1}\right)\left[\begin{array}{c}
0 \\
B_{m}
\end{array}\right]=M\left[\begin{array}{c}
0 \\
B_{m}
\end{array}\right]
$$

Reorganizing the Eq. (3.58), we obtain 


$$
\left[\begin{array}{ll}
\frac{u_{1}^{2}}{n_{t}^{2}} J_{v}\left(u_{1} r_{1}\right) & M_{12} \\
u_{1} J_{v}^{\prime}\left(u_{1} r_{1}\right) & M_{22}
\end{array}\right]\left[\begin{array}{c}
-A_{1} \\
B_{m}
\end{array}\right]=0
$$

The dispersion equation of TM modes can be written as

$$
\frac{u_{1}}{n_{t}^{2}} J_{v}\left(u_{1} r_{1}\right) M_{22}-J_{v}^{\prime}\left(u_{1} r_{1}\right) M_{12}=0
$$

Solving the dispersion equation of TM modes, the effective index of each mode can be obtained. The field distributions of the mode can be calculated from Eqs. (3.43-3.46) by applying the field coefficients $C_{t}=D_{t}=0$.

\subsection{Linear Polarization Modes in Multilayer Step-Index Optical Fibres}

Most of single mode fibres used in telecommunications are weakly guided optical fibres. There are approximate solutions of Maxwell equations in this case, and the fibre modes are linearly polarized. Also in most cases, the weakly guided conditions can not be obeyed in multilayer step-index fibres, but if the fibre core layer and its nearby cladding layer satisfy the weakly guided condition, for core mode and low order cladding modes, the linearly polarization (LP) modes will still be a good approximation. As each LP mode is some combination of HE/EH and TE/TM modes, therefore using LP mode can simplify the mode coupling calculation especially in tilted FBG.

In LP modes, the transverse electric field and its derivative respect to radius are:

For $n_{c f f}<n_{t}$,

$$
\left[\begin{array}{l}
e_{t} \\
\frac{d e_{t}}{d r}
\end{array}\right]=\left[\begin{array}{cc}
J_{v}\left(u_{t} r\right) & Y_{v}\left(u_{t} r\right) \\
u_{t} J_{v}^{\prime}\left(u_{t} r\right) & u_{t} Y_{v}^{\prime}\left(u_{t} r\right)
\end{array}\right]\left[\begin{array}{l}
A_{t} \\
B_{t}
\end{array}\right]=M_{t}\left[\begin{array}{l}
A_{t} \\
B_{t}
\end{array}\right]
$$


where $t=x, y$, the inverse matrix of $M_{i}$

$$
M_{i}^{-1}=\frac{\pi r}{2}\left[\begin{array}{cc}
u_{i} Y_{v}^{\prime}\left(u_{t} r\right) & -Y_{v}\left(u_{t} r\right) \\
-u_{t} J_{v}^{\prime}\left(u_{t} r\right) & J_{v}\left(u_{t} r\right)
\end{array}\right]
$$

For $n_{e f f}>n_{i}$

$$
M_{t}=\left[\begin{array}{cc}
I_{v}\left(w_{t} r\right) & K_{v}\left(w_{i} r\right) \\
w_{1} I_{v}^{\prime}\left(w_{i} r\right) & w_{t} K_{v}^{\prime}\left(w_{t} r\right)
\end{array}\right]
$$

And its inverse matrix

$$
M_{i}^{-1}=\frac{1}{w_{i}\left(I_{v}^{\prime}\left(w_{t} r\right) K_{v}\left(w_{t} r\right)-I_{v}\left(w_{t} r\right) K_{v}^{\prime}\left(w_{i} r\right)\right)}\left[\begin{array}{cc}
w_{i} K_{v}^{\prime}\left(w_{t} r\right) & -K_{v}\left(w_{t} r\right) \\
-w_{i} I_{v}^{\prime}\left(w_{t} r\right) & I_{v}\left(w_{t} r\right)
\end{array}\right]
$$

For LP modes, the transverse electric field and its derivative to radius are continuous at the boundary [18]. Applying the boundary conditions for each layer, we obtain

$$
M_{1}\left(r_{1}\right)\left[\begin{array}{c}
A_{1} \\
0
\end{array}\right]=M_{2}\left(r_{1}\right) M_{2}^{-1}\left(r_{2}\right) M_{3}\left(r_{2}\right) M_{3}^{-1}\left(r_{3}\right) \cdots M_{m-1}^{-1}\left(r_{m-1}\right) M_{m}\left(r_{m-1}\right)\left[\begin{array}{c}
0 \\
B_{m}
\end{array}\right]=M\left[\begin{array}{c}
0 \\
B_{m}
\end{array}\right]
$$

Reorganizing the Eq. (3.65), we obtain

$$
\left[\begin{array}{cc}
J_{v}\left(u_{1} r_{1}\right) & M_{12} \\
u_{1} J_{v}^{\prime}\left(u_{1} r_{1}\right) & M_{22}
\end{array}\right]\left[\begin{array}{c}
-A_{1} \\
B_{m}
\end{array}\right]=0
$$

The dispersion equation of LP modes can be written as

$$
J_{v}\left(u_{1} r_{1}\right) M_{22}-u_{1} J_{v}^{\prime}\left(u_{1} r_{1}\right) M_{12}=0
$$

After solving the dispersion equation of LP modes, the effective index of each mode can be obtained. The field distributions of LP modes can be calculated as well [35]. 


\subsection{Solving the Dispersion Equations for Lossless Optical Fibres}

How to accurately and efficiently solve the dispersion equations is always a big challenge for numerical simulations. There are several numerical ${ }_{\star}$ nethods can be used to solve the dispersion equations for the guided modes in lossless optical waveguides, such as Bisection Method, Newton's Method and Muller's Method [40]. The Bisection Method is reliable which can find all the modes but it is slow; the Newton's Method is more efficient but needs to calculate the derivative of the dispersion equation and an initial guess value for the effective index; Muller's Method also needs an initial guess value but is very efficient. To efficiently solve the dispersion equations of HE/EH modes, TE/TM modes and LP modes, the algorithm used here combines the Bisection Method and Muller's Method. First, we use the Bisection Method to approximately find the roots of dispersion equations (to save the root found time), and then we use Muller's Method to polish the root as accurately as wanted.

\subsection{Solving the Dispersion Equations for Lossy and Gain Optical Fibres}

Finding the complex roots of the dispersion equations is more difficult than for real ones. Instead of one dimension scanning for real roots (in this case, the root searching can be performed from the maximum refractive index of the fibre to the minimum refractive index of fibre), now a two dimensional scanning is needed for complex roots. There are infinite directions for searching the complex root due to both real and imaginary parts of the root changes.

Both Newton's Method and Mullcr's Method work for complex roots as well, but both methods need an initial guess valuc. The accuracy of the guess value determines 
where the root converges, meaning that you may not get the root that you wanted. In some cases, the perturbation method can be used by choosing the roots found in lossless media as guess values, but it is not reliable to converge to the roots in all cases.

There is a reliable method that not only can solve complex roots of dispersion equations but that can find all the roots within a enclosed region as well, it is the Cauchy`s Integration Method or Argument Principle Method [41]. It was successfully used to solve the dispersion equations of TE/TM modes in planar optical waveguides $[36,37]$.

\subsubsection{Argument Principle Method}

The APM [39] is based on the argument principle [42] and the residue theorem of complex analysis. If a function $f(z)$ is analytic and does not go to zero over a closed integral contour, then the argument principle is of the form:

$$
S_{0}=-\frac{1}{.2 \pi}\left[\int\right] \frac{f^{\prime}(z)}{f(z)} d z=N_{z}-N_{p}
$$

where $N_{z}$ is the number of zeros and $N_{p}$ is number of poles inside the region enclosed by the contour $C$. If there arc no poles in the region enclosed by $C$, then $S_{0}$ is the number of zeros, and from the residue theorem we have:

$$
S_{m}=\frac{1}{j 2 \pi}\left[\int_{i} z^{m} \frac{f^{\prime}(z)}{f(z)} d z=\sum_{i=1}^{S_{0}} z_{i}^{m} \quad \text { for } m=1,2, \cdots, S_{0}\right.
$$

where $z_{i}, i=1,2, \ldots, S_{0}$ are the roots of $f(z)$ inside $C$ and $S_{m}$ is the sum of $z_{t}^{m}$ with $m=1$, $2, \ldots, S_{0}$. Eq. (3.69) leads to a system of equations that can be used to evaluate the 
coefficients of a polynomial $p(z)$ of degree $S_{0}$, which has the same roots $z_{1}, \cdots, z_{s_{0}}$ as the function $f(z)$ inside $C$. The approximation polynomial $p(z)$ can be written as:

$$
p(z)=\prod_{i=1}^{s_{0}}\left(z-z_{\imath}\right)=\sum_{k=1}^{s_{0}} C_{k} z^{k}
$$

with $C_{S_{0}}=1$. The coefficients $C_{k}$ are given via Newtōn's recursive formula:

$$
C_{k}=\frac{1}{\left(k-S_{0}\right)} \sum_{j=1}^{S_{0}-k} S, C_{k+1} \quad \text { for } k=S_{0}-1, \cdots, 0
$$

The polynomial $p(z)$ can be solved by standard techniques such as Laguerre's Method [10]. The problem of finding the zeros of an arbitrary function $f(z)$ is thus transformed to the simpler problem of finding the zeros of the polynomial $p(z)$, for which a variety of reliable and efficient numerical methods exist.

The roots of polynomial $p(z)$ and $f(z)$ do not coincide due to the integration errors introduced by Eq. (3.69). First $S_{0}$ should be confined to $S_{0} \leq 4$, This ensures that the degree and the coefficients of the polynomial $p(z)$ remain small, thus helping to reduce numerical errors when locating the rovis. Then, after the roots of the poiynomial $p(z)$ are obtained. A further refinement must be performed to find the roots of $f(z)$ by applying Muller's Miethod with the initial guess being the ronts of $p(z)$.

\subsubsection{Derivative of Dispersion Equations of Hybrid Modes}

The Cauchy's Integration Method necd calculate the derivative of the dispersion equation. The derivatives of the HE/EH modes dispersion equations and the derivative of each term of the matrix $M_{1}$ and its interse matrix $M_{1}^{-1}$ are derived. The derivative of dispersion equations for the TE/TM modes and LP mode are much simpler and can be 
directly derived from $\mathrm{HE} / \mathrm{EH}$ modes'. The derivatives of the $i \mathrm{E} / \mathrm{EH}$ modes dispersion equation are as follows:

$$
\frac{d F}{d n_{e f f}}=\frac{d a}{d n_{e f f}} x^{2}+2 a x \frac{d x}{d n_{e f f}}+\frac{d b}{d n_{e f f}} x+b \frac{d x}{d n_{e f f}}+\frac{d c}{d n_{e f f}}
$$

where

$$
\begin{aligned}
\frac{d a}{d n_{e f f}}= & \frac{d M_{13}}{d n_{e f f}} M_{31}+M_{13} \frac{d M_{31}}{d n_{e f f}}-\frac{d M_{11}}{d n_{e f f}} M_{33}-M_{11} \frac{d M_{33}}{d n_{e f f}} \\
\frac{d b}{d n_{e f f}}= & \frac{d M_{11}}{d n_{e f f}} M_{43}+M_{11} \frac{d M_{43}}{d n_{e f f}}-\frac{d M_{13}}{d n_{e f f}} M_{41}-M_{13} \frac{d M_{41}}{d n_{e f f}} \\
& +\frac{d M_{21}}{d n_{e f f}} M_{35}+M_{21} \frac{d M_{33}}{d n_{e f f}}-\frac{d M_{23}}{d n_{e f f}} M_{31}-M_{23} \frac{d M_{31}}{d n_{e f f}} \\
\frac{d c}{d n_{e f f}}= & \frac{d M_{23}}{d n_{e f f}} M_{41}+M_{23} \frac{d M_{41}}{d n_{e f f}}-\frac{d M_{2}}{d n_{e f f}} M_{43}-M_{21} \frac{d M_{43}}{d n_{e f f}} \\
\frac{d x}{d n_{e f f}}= & -\frac{k^{2} n_{e f f}}{w_{m}^{2}}\left[\frac{K_{v-1}\left(w_{m m} r_{m-1}\right)+K_{v+1}\left(w_{m} r_{m-1}\right)}{2 K_{v}\left(w_{m m} r_{m-1}\right)}-\frac{K_{v}^{\prime}\left(w_{m} r_{m-1}\right)}{w_{e n} K_{v}\left(w_{m} r_{m-1}\right)}-r_{m-1}\left(\frac{K_{v}^{\prime}\left(w_{m} r_{m-1}\right)}{K_{v}\left(w_{m} r_{m-1}\right)}\right)^{2}\right]
\end{aligned}
$$

Derivatives for each term in matrix $M_{i}$ and its inverse matrix $M_{t}^{-1}$ are as follows:

If $n_{i}>n_{c f f}$

$$
\begin{aligned}
& \beta=\frac{2 \pi}{\lambda} n_{f f f}=k n_{e f f} \\
& u_{t}^{2}=k^{2} n_{i}^{2}-\beta^{2}=k^{2}\left(n_{t}^{2}-n_{c f f}^{2}\right) \\
& \frac{d u_{t}}{d n_{c f f}}=-\frac{k^{2} n_{c f f}}{u_{t}} \\
& \frac{d}{d n_{c f f}}\left(\frac{\sigma_{1}}{u_{r}^{2} r}\right)=\frac{\sigma_{1}}{u_{i}^{2} r}\left(\frac{1}{n_{f f l}}+\frac{2 k^{2} n_{e f f}}{u_{t}^{2}}\right)
\end{aligned}
$$




$$
\begin{aligned}
& \frac{d}{d n_{e f f}}\left(\frac{\sigma_{2}}{u_{t}^{2} r n_{t}^{2}}\right)=\frac{\sigma_{2}}{u_{t}^{2} r n_{t}^{2}}\left(\frac{1}{n_{e f f}}+\frac{2 k^{2} n_{e f f}}{u_{t}^{2}}\right) \\
& \frac{d\left[u_{t}^{2} J_{v}\left(u_{t} r\right)\right]}{d n_{e f f}}=-k^{2} n_{e f f} r\left[2 J_{v}\left(u_{t} r\right)+u_{t} J_{v}^{\prime}\left(u_{t} r\right)\right] \\
& \frac{d\left[u_{t}^{2} Y_{v}\left(u_{t} r\right)\right]}{d n_{e f f}}=-k^{2} n_{e f f} r\left[2 Y_{v}\left(u_{t} r\right)+u_{t} Y_{v}^{\prime}\left(u_{i} r\right)\right] \\
& \frac{d\left[u_{t} J_{v}^{\prime}\left(u_{t} r\right)\right]}{d n_{e f f}}=-k^{2} n_{e f f} r\left[\frac{J_{v}^{\prime}\left(u_{t} r\right)}{u_{t} r}+\frac{J_{v-1}^{\prime}\left(u_{t} r\right)}{2}-\frac{J_{v+1}^{\prime}\left(u_{t} r\right)}{2}\right] \\
& \frac{d\left[u_{t} Y_{v}^{\prime}\left(u_{t} r\right)\right]}{d n_{e f f}}=-k^{2} n_{e f f} r\left[\frac{Y_{v}^{\prime}\left(u_{t} r\right)}{u_{t} r}+\frac{Y_{v-1}^{\prime}\left(u_{t} r\right)}{2}-\frac{Y_{v+1}^{\prime}\left(u_{t} r\right)}{2}\right]
\end{aligned}
$$

If $n_{\iota}<n_{c f f}$

$$
\begin{aligned}
& w_{t}^{2}=\beta^{2}-k^{2} n_{t}^{2}=k^{2}\left(n_{e f f}^{2}-n_{i}^{2}\right) \\
& \frac{d w_{t}}{d n_{e f f}}=\frac{k^{2} n_{e f f}}{w_{t}} \\
& \frac{d}{d n_{e f f}}\left(\frac{\sigma_{1}}{w_{i}^{2} r}\right)=\frac{\sigma_{1}}{w_{t}^{2} r}\left(\frac{1}{n_{e f f}}-\frac{2 k^{2} n_{e f f}}{w_{t}^{2}}\right) \\
& \frac{d}{d n_{e f f}}\left(\frac{\sigma_{2}}{w_{t}^{2} r n_{t}^{2}}\right)=\frac{\sigma_{2}}{w_{t}^{2} r n_{t}^{2}}\left(\frac{1}{n_{e f f}}-\frac{2 k^{2} n_{e f f}}{w_{t}^{2}}\right) \\
& \frac{d\left[w_{t}^{2} I_{v}\left(w_{t} r\right)\right]}{d n_{e f f}}=k^{2} n_{e f f} r\left[2 I_{v}\left(w_{t} r\right)+w_{t} I_{v}^{\prime}\left(w_{1} r\right)\right] \\
& \frac{d\left[w_{t}^{2} K_{v}\left(w_{t} r\right)\right]}{d n_{e f f}}=k^{2} n_{e f f} r\left[2 K_{v}\left(w_{t} r\right)+w_{t} K_{v}^{\prime}\left(w_{t} r\right)\right] \\
& \frac{d\left[w_{t} I_{v}^{\prime}\left(w_{t} r\right)\right]}{d n_{e f f}}=k^{2} n_{e f f} r\left[\frac{I_{v}\left(w_{t} r\right)}{w_{t} r}+\frac{I_{v-1}\left(w_{t} r\right)}{2}+\frac{I_{v+1}^{\prime}\left(w_{t} r\right)}{2}\right]
\end{aligned}
$$




$$
\begin{aligned}
& \frac{d\left[w_{1} K_{v}^{\prime}\left(w_{1} r\right)\right]}{d n_{e f f}}=k^{2} n_{e f f} r\left[\frac{K_{v}^{\prime}\left(w_{i} r\right)}{w_{t} r}-\frac{K_{v-1}^{\prime}\left(w_{i} r\right)}{2}-\frac{K_{v+1}^{\prime}\left(w_{t} r\right)}{2}\right] \\
& \frac{d}{d n_{e f f}}\left[-\frac{1}{w_{1}^{3}} \frac{1}{I_{v}\left(w_{1} r\right) K_{v+1}\left(w_{1} r\right)+I_{v+1}\left(w_{t} r\right) K_{v}\left(w_{t} r\right)}\right]=\frac{k^{2} n_{e f f}}{w_{t}^{5}} \frac{1}{I_{1}\left(w_{t} r\right) K_{t+1}\left(w_{1} r\right)+I_{v+1}\left(w_{t} r\right) K_{v}(w, r)} \\
& +\frac{k^{2} n_{e f f} r}{w_{t}^{4}} \frac{\left(I_{v}^{\prime}\left(w_{t} r\right) K_{t+1}\left(w_{t} r\right)+I_{v}\left(w_{t} r\right) K_{t+1}^{\prime}\left(w_{1} r\right)+I_{1+1}^{\prime}\left(w_{t} r\right) K_{v}\left(w_{t} r\right)+I_{1+1}\left(w_{t} r\right) K_{v}^{\prime}\left(w_{1} r\right)\right)}{\left[I_{1}\left(w_{1} r\right) K_{1+1}\left(w_{1} r\right)+I_{i+1}\left(w_{t} r\right) K_{1}\left(w_{t} r\right)\right]^{2}}
\end{aligned}
$$

\subsubsection{Algorithm for Computing the Derivative of the Transfer Matrices}

The transfer matrices and their derivatives as expressed by Eqs. (3.76-3.85) can be computed in an efficient manner [37]. The idea is to accumulate the derivative of the transfer matrix as the latter is being constructed. Consider accumulation over the first 3 layers of a waveguide structure. For the first layer $(i=1), M_{1}$ and $\frac{d M_{1}}{d n_{e f f}}$ are computed. For the second layer $(i=2), \quad M_{2}$ and $\frac{d M_{2}}{d n_{d f f}}$ are computed, then $\frac{d M}{d n_{\text {eff }}}=\frac{d\left(M_{1} M_{2}\right)}{d n_{\text {eff }}}=M_{1} \frac{d M_{2}}{d n_{\text {eff }}}+\frac{d M_{1}}{d n_{\text {eff }}} M_{2}$ and $M=M_{1} M_{2}$ are accumulated. For the third layer $(i=3), M_{3}$ and $\frac{d M_{3}}{d n_{e f f}}$ are computed, then $\frac{d M}{d n_{e f f}}=\frac{d\left(M_{1} M_{2} M_{3}\right)}{d n_{c f f}}=\frac{d\left(M_{1} M_{2}\right)}{d n_{e f f}} M_{3}+\left(M_{1} M_{2}\right) \frac{d M_{3}}{d n_{e f f}}$ and $M=M_{1} M_{2} M_{3}$ are accumulated. The accumulation process continues until all layers in the structure have been handled. Clearly, one matrix product is required per layer to accumulate the transfer matrix and two products are required to accumulate its derivative. This algorithm thus accumulates the transfer matrix for $m$ layers in $m$ matrix products and the derivative of the transfer matrix in $2 m$ matrix products. 


\subsection{Numerical Results}

\subsubsection{Core and Cladding Modes in SMF-28 Fibre}

The SMF-28 fibre crosssection is shown in Fig. 3.2. The refracive indices for core, cladding and surrounding layers are $1.448702,1.444024$ and 1.0 respectively at $\lambda=1.55$ $\mu \mathrm{m}$, which are calculated by using Sellmeier equation, and the Sellmeier parameters are obtained from OptiGrating [43], the radius for the core and cladding layer are $4.15 \mu \mathrm{m}$ and $62.5 \mu \mathrm{m}$. The Table 3.1 shows the effective indices of first $32 \mathrm{HE}_{1 \mathrm{~m}}, \mathrm{EH}_{1 \mathrm{~m}}, \mathrm{HE}_{2 \mathrm{~m}}$, $\mathrm{EH}_{2 \mathrm{~m}}, \mathrm{TE}_{0 \mathrm{~m}} \mathrm{TM}_{0 \mathrm{~m}}, \mathrm{LP}_{0 \mathrm{~m}}$ and $\mathrm{LP}_{1 \mathrm{~m}}$ modes. The calculations of the roots (effective indices) of dispersion equation is first made using the Bisection Method to the accuracy of 6 digits for effective index, and then polished by Muller's Method to a relative error less than $10^{-20}$. The results can be validated by comparing the effective indices of $\mathrm{LP}_{01}$ and $\mathrm{HE}_{11}$ modes. It can be seen from the Table 3.1 that the effective index of $\mathrm{LP}_{01}$ and the effective index of $H E_{11}$ are well matched.

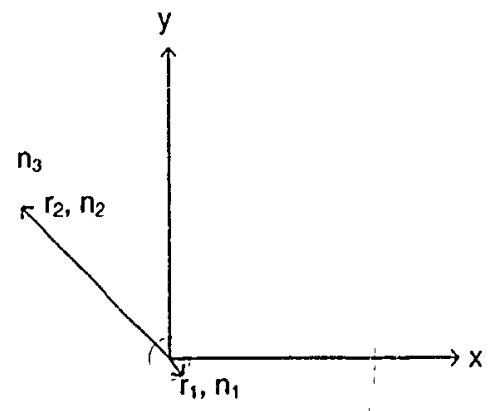

Figure 3.2: 'The cross-section of SMF-28 fibre 
Table 3.1: The effective index of different fibre modes in SMF-28 fibre

\begin{tabular}{|c|c|c|c|c|c|c|c|c|}
\hline $\mathrm{m}$ & $1 \mathrm{~m}$ & EF & $\mathrm{HE}$ & $m$ & $m$ & $m$ & $m$ & $\pi$ \\
\hline i & 20 & & & & & & & \\
\hline 2 & 582 & 41 & 1 & 786 & 92 & 35 & 3 & 1. \\
\hline 3 & & & & & & & & \\
\hline 4 & 6 & 1 & 1 & 5 & 11 & 7 & 9 & 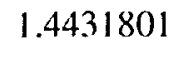 \\
\hline 5 & 95 & & & & & & & \\
\hline 6 & & & & & & 6 & & 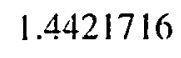 \\
\hline 7 & 6 & 97 & 53 & 66 & 51 & 51 & & \\
\hline 8 & & & & 53 & & 93 & 56 & \\
\hline 9 & & & 1.4 & & & & & \\
\hline 10 & 7 & 8 & & & & & & \\
\hline 1 & & & & & & & & \\
\hline 12 & & & & & & & & \\
\hline 13 & & & & & & & & \\
\hline 14 & & & & & & & & \\
\hline 15 & & & & & & & & \\
\hline 10 & & & & & & & & \\
\hline 17 & & & & & & & & \\
\hline 1 & & & & & & & & \\
\hline 19 & & & & & & & & \\
\hline 20 & & & & & & & & \\
\hline 2 & & & & & & & & \\
\hline 22 & & & & & & & & 1 \\
\hline 23 & & & & & & & & \\
\hline 24 & & & & & & & & \\
\hline 25 & & & & & & & & \\
\hline 26 & & & & & & & & \\
\hline 27 & & & & & & & & \\
\hline 28 & . & 31 & 1.2 & 92 & & & & \\
\hline 2 & & 51 & & & & & & \\
\hline$x$ & I. & 16 & 1. & 1. & 1. & 1. & 1. & 1. \\
\hline$\vec{\theta}$ & & 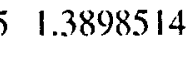 & & 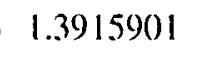 & & & & 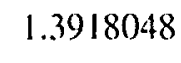 \\
\hline 32 & 1.3900654 & 1.3863022 & 1.3846997 & 1.3880873 & 1.388 .3122 & 1.3880899 & 1.3900540 & 1.3883122 \\
\hline
\end{tabular}




\begin{tabular}{llllllllll}
33 & 1.3865317 & 1.3826319 & 1.3809750 & 1.3844668 & 1.3847007 & 1.3844656 & 1.3865200 & 1.3847007 \\
34 & 1.3828774 & 1.3788387 & 1.3771325 & 1.380728 & 1.3809709 & 1.3807225 & 1.3828665 & 1.3809709 \\
35 & 1.3791003 & 1.3749210 & 1.3731713 & 1.3708702 & 1.3771227 & 1.3768603 & 1.3790910 & 1.3771227 \\
36 & 1.3751988 & 1.3708779 & 1.3690898 & 1.3728923 & 1.3731560 & 1.3728787 & 1.3751915 & 1.3731560 \\
37 & 1.3711719 & 1.3667087 & 1.3648859 & 1.368793 & 1.3690698 & 1.3687765 & 1.3711665 & 1.3690698 \\
38 & 1.3670187 & 1.3624129 & 1.3605576 & 1.3645706 & 1.3648629 & 1.3645523 & 1.3670149 & 1.3648629 \\
39 & 1.3627388 & 1.3579904 & 1.3561024 & 1.3602232 & 1.3605332 & 1.3602042 & 1.3627361 & 1.3605332 \\
40 & 1.3583322 & 1.3534408 & 1.3515182 & 1.3557486 & 1.3560785 & 1.3557300 & 1.3583299 & 1.3560785 \\
\hline
\end{tabular}

\subsubsection{Cladd'ng Modes in SMF Fibre with Nano-Size Metal Coating}

Some cladding modes in a fibre with a metal coating will show surface plasmon wave characteristics. We present here our calculation for a fibre that has been studied elsewhere (as an additional validation of our model and of its numerical implementation). The four layer fibre has a $36 \mathrm{~nm}$ metal coating and the parameters: $n_{1}=1.4602, n_{2}=1.4443, n_{3}=0.00298-j 13.4857, n_{4}=1.36061, \quad r_{1}=2.25 \mu \mathrm{m}$, $r_{2}=62.5 \mu \mathrm{m}, r_{3}=62.536 \mu \mathrm{m}(d=36 \mathrm{~nm})$, and $\lambda=1.55 \mu \mathrm{m}[44]$ as shown in Fig. 3.3. The effective indices of the hybrid modes are calculated. There are 76 cladding modes, the $8 \mathrm{HE}_{1 \mathrm{~m}} / \mathrm{EH}_{1 \mathrm{~m}}$ cladding modes discussed in reference [44] are listed in table 3.2, and their azimuthal magnetic fields are shown in Fig. 3.4 (a) and (b). The fields of each mode are normalized to $P_{i}=1 \mathrm{~W}$. Fields shown in Fig. $3.5(\mathrm{c})$ and $(\mathrm{d})$ are good agreement with the Fig. 3.5 (a) and (b) from reference [44]. The difference of the field amplitudes is caused by the method used to calculation the integration of the power $P_{-}^{\prime}$, here we use the conjugate form for the power normalization and they use the unconjugate form. 


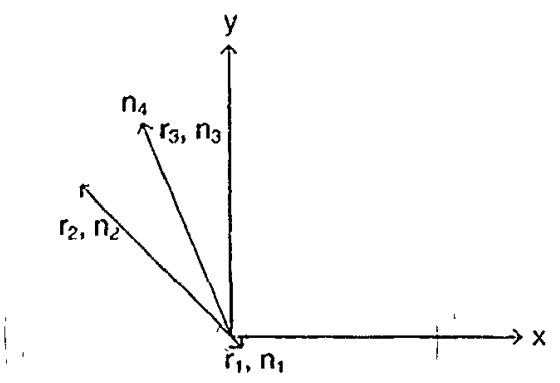

Figure 3.3: The cross-section of SMF fibre with $36 \mathrm{~nm}$ metal coating

Table 3.2: The effective index of different fibre modes in SMF-28 fibre

\begin{tabular}{cccc}
\hline Mode & Effective Index & Mode & Effective l: dex \\
\hline 68 & $1.3752521393-1.0763175837^{*} 10^{-8} \mathrm{j}$ & 69 & $1.3737224400-2.9920850504^{*} 10^{-7} \mathrm{j}$ \\
70 & $1.3711960448-1.1490567111^{*} 10^{-8} \mathrm{j}$ & 71 & $1.3698078791-1.0145518518^{*} 10^{-6} \mathrm{j}$ \\
72 & $1.3670129491-1.2225313751^{*} 10^{-8} \mathrm{j}$ & SPW & $1.3680420493-2.6040631406^{*} 10^{-6} \mathrm{j}$ \\
74 & $1.3627022280-1.2828951588^{*} 10^{-8} \mathrm{j}$ & 73 & $1.3651252046-7.3091938257^{*} 10^{-8} \mathrm{j}$ \\
\hline
\end{tabular}




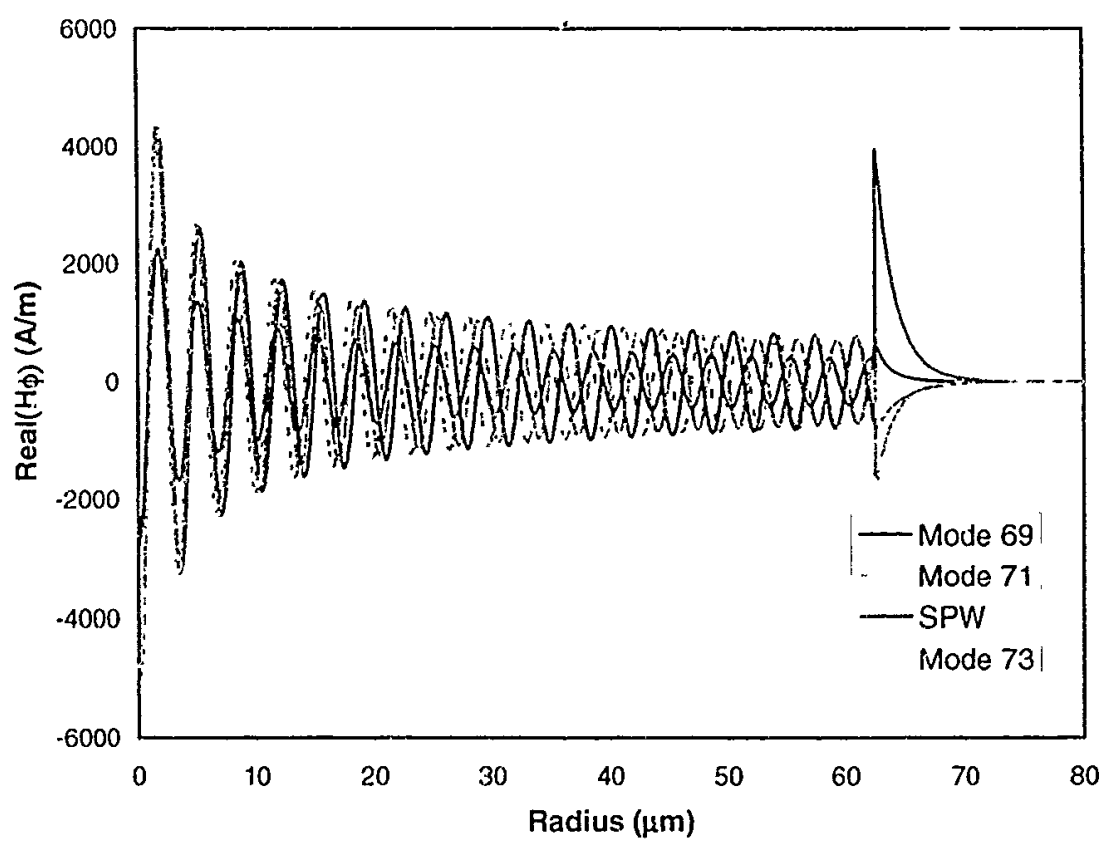

(a)

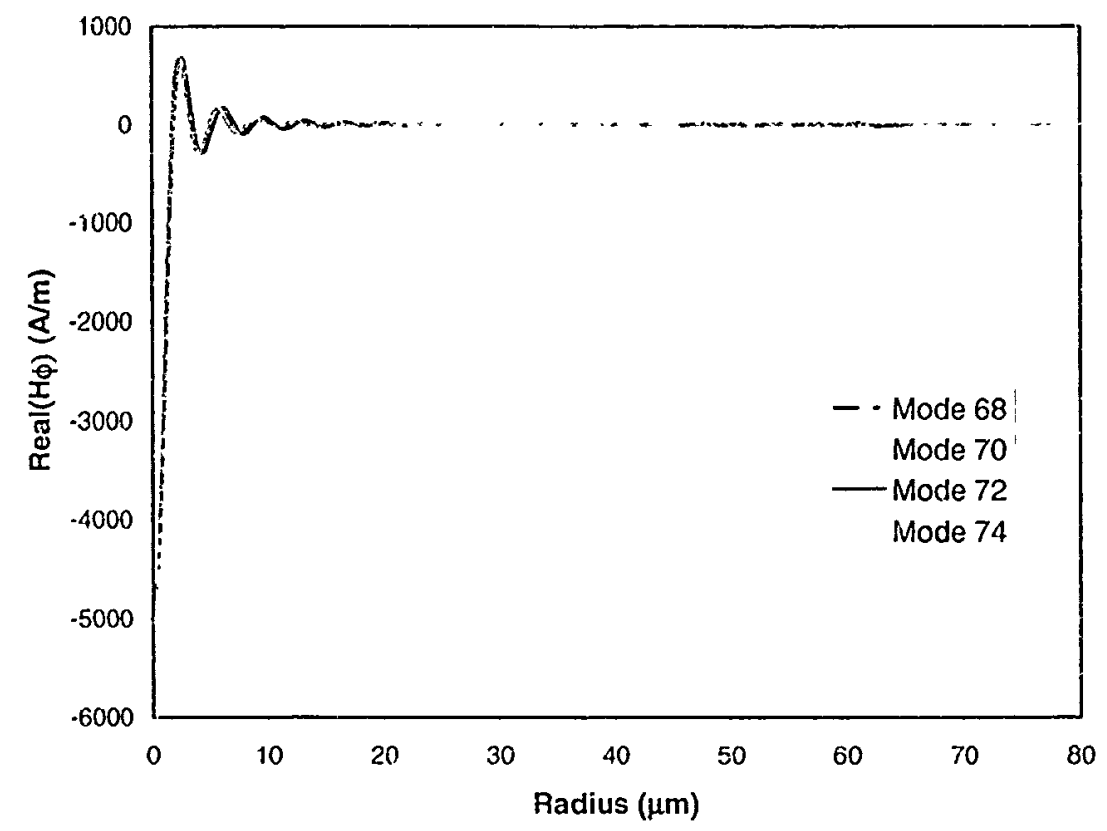

(b)

Figure 3.4: The normalized azimuthal magnetic fields of cladding modes: (a) odd modes, (b) even modes 

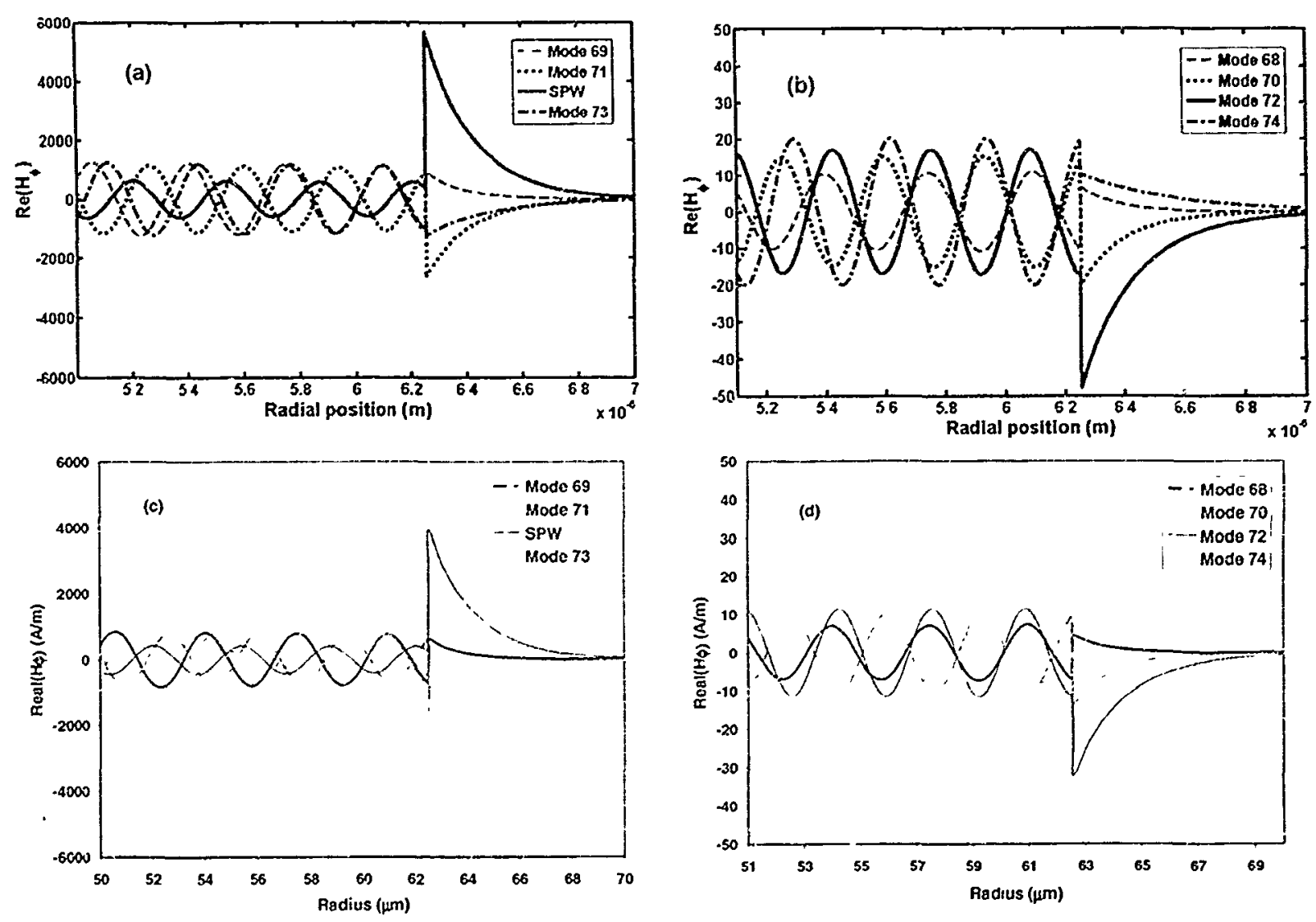

Figure 3.5: The azimuthal magnetic fields of cladding modes: (a) odd modes; (b) even modes from reference [44]; (c) odd modes, and (d) even modes are simulated.

\subsubsection{The Plasmon Mode in Thinned SMF fibre with a Thin Gold Coating}

Some cladding modes in the fibre with a metal coating will show surface plasmon wave characteristics as shown in Fig. 3.5. This is characterized by large field amplitude at the metal boundary. There is also a pure plasmon mode that can exists in the fibre with metal coating, because metals at light wavelengths have the necessary conditions for plasmon formation (negative real part of dielectric constant). The plasmon mode has the largest effective index of all guided and leaky modes and its effective index is larger than fibre corc and cladding indices. Its field is only confined at metal surfaces. This mode is very 
useful for the refractive index sensing applications due its extremely high index sensitivity to the surrounding media. Here we calculate the cffective index and field distributions of such mode. The four layer fibre which has a $20 \mathrm{~nm}$ gold coating has the parameters: $n_{1}=1.4504, n_{2}=1.444024, n_{3}=0.55-j 11.5, n_{4}=1.0, r_{1}=4.15 \mu \mathrm{m}$, $r_{2}=12.5 \mu \mathrm{m}, r_{3}=12.52 \mu \mathrm{m}(d=20 \mathrm{~nm})$, and $\lambda=1.55 \mu \mathrm{m}$ as shown in Fig. 3.6. The effective index, the loss and the confinement at the surrounding layer of the plasmon mode with different surrounding refractive index are listed in Table 3.3 , and its field distributions are shown in Fig. 3.7. From table 3.3 we can see that the plasmon mode has an extremely large loss that is comparable with the loss of plasmon mode in planar optical waveguides which were shown in reference [37]. It also can be sqen that as the SRI increases, the loss and the power in the surrounding layer are increasing as well. From Fig. 3.7, it is clear that as the SRI increases, the field penetrates more strongly on the external side of the gold surface.

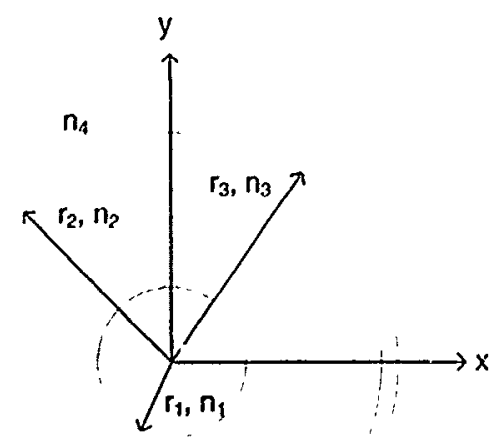

Figure 3.6: The cross-section of thinned SMF fibre with $25 \mathrm{~nm}$ gold coating 
Table 3.3: The effective index of different fibre modes in thinned SMF fibre

\begin{tabular}{ccccc}
\hline$n_{A}$ & Effective index & Loss (dB/mm) & $\Gamma$ \\
\hline 1.00 & $1.465319194466308-3.778776928495299^{*} 10^{-3} \mathrm{j}$ & 133.05 & $0.36 \%$ \\
1.30 & $1.471150158359611-5.695691743112360^{*} 10^{-3} \mathrm{j}$ & 200.55 & $3.96 \%$ \\
1.36 & $1.475965819964934-7.151722522997441^{*} 10^{-3} \mathrm{j}$ & 251.81 & $10.19 \%$ \\
1.40 & $1.489913946060439-9.681158191857822^{*} 10^{-3} \mathrm{j}$ & 340.87 & $34.97 \%$ \\
\hline
\end{tabular}

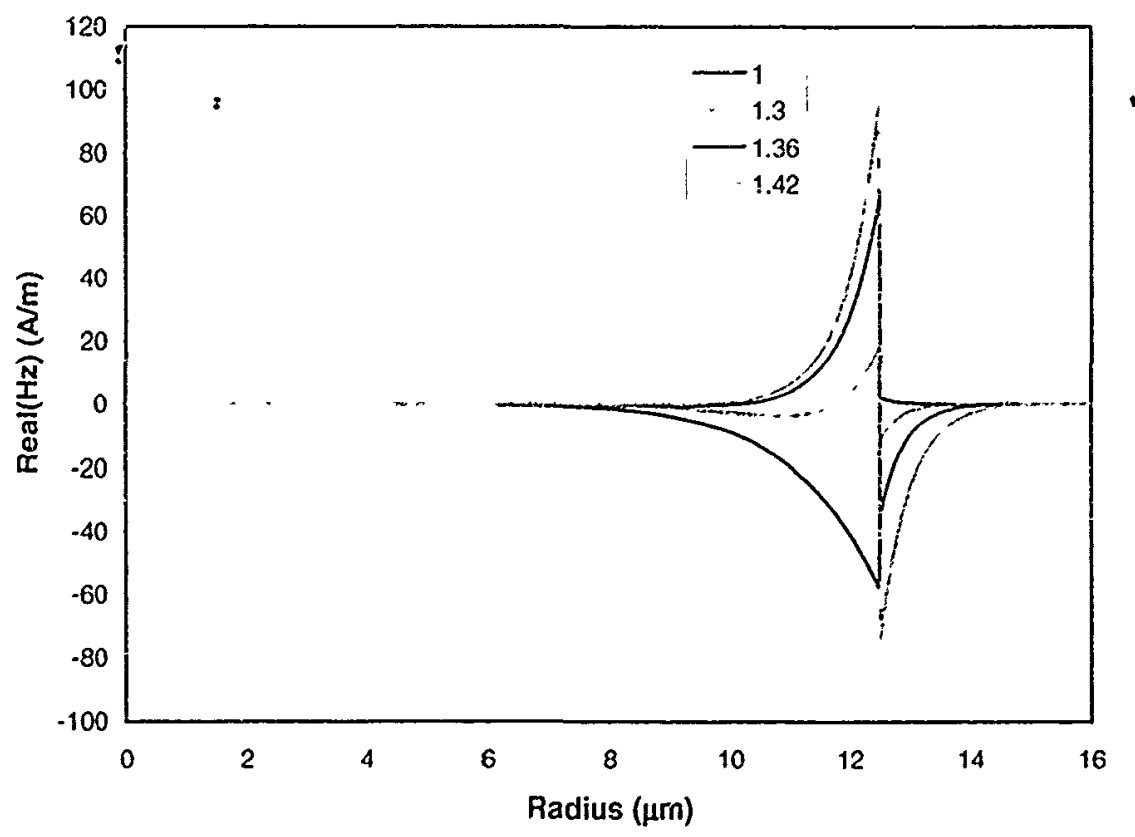

(a) 


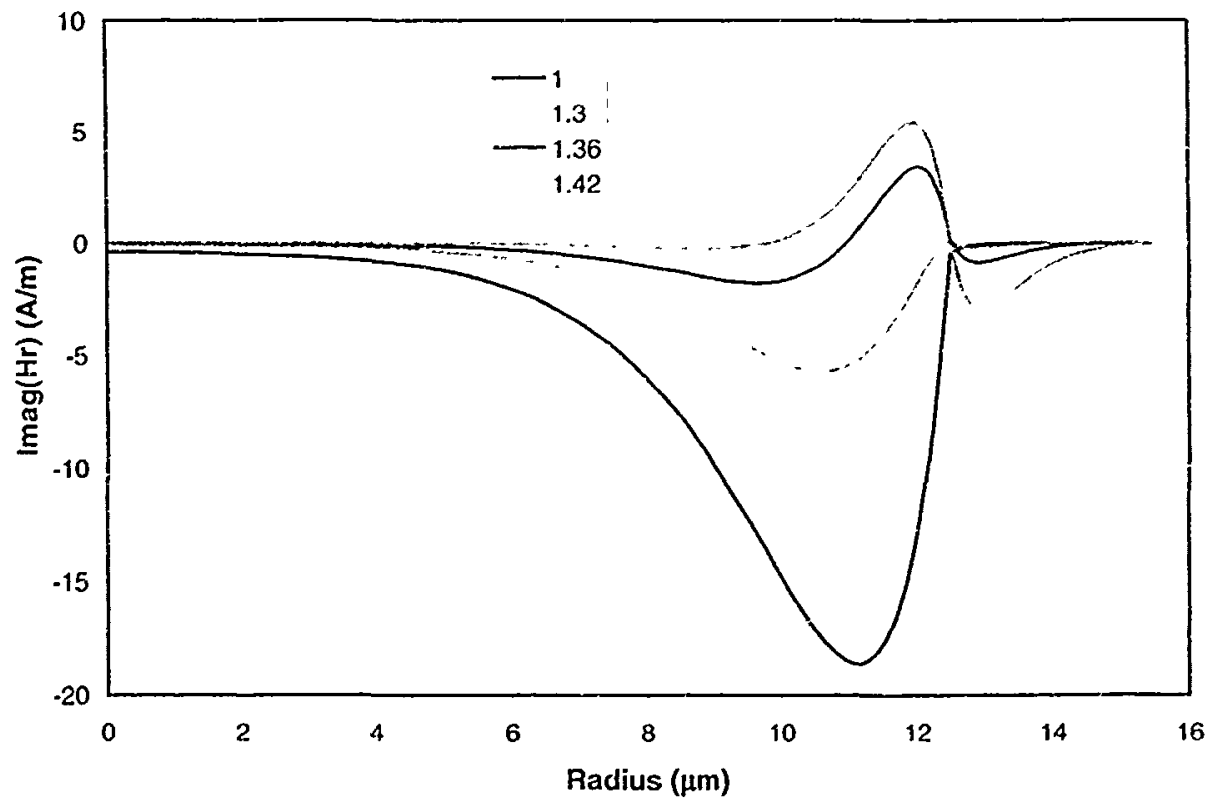

(b)

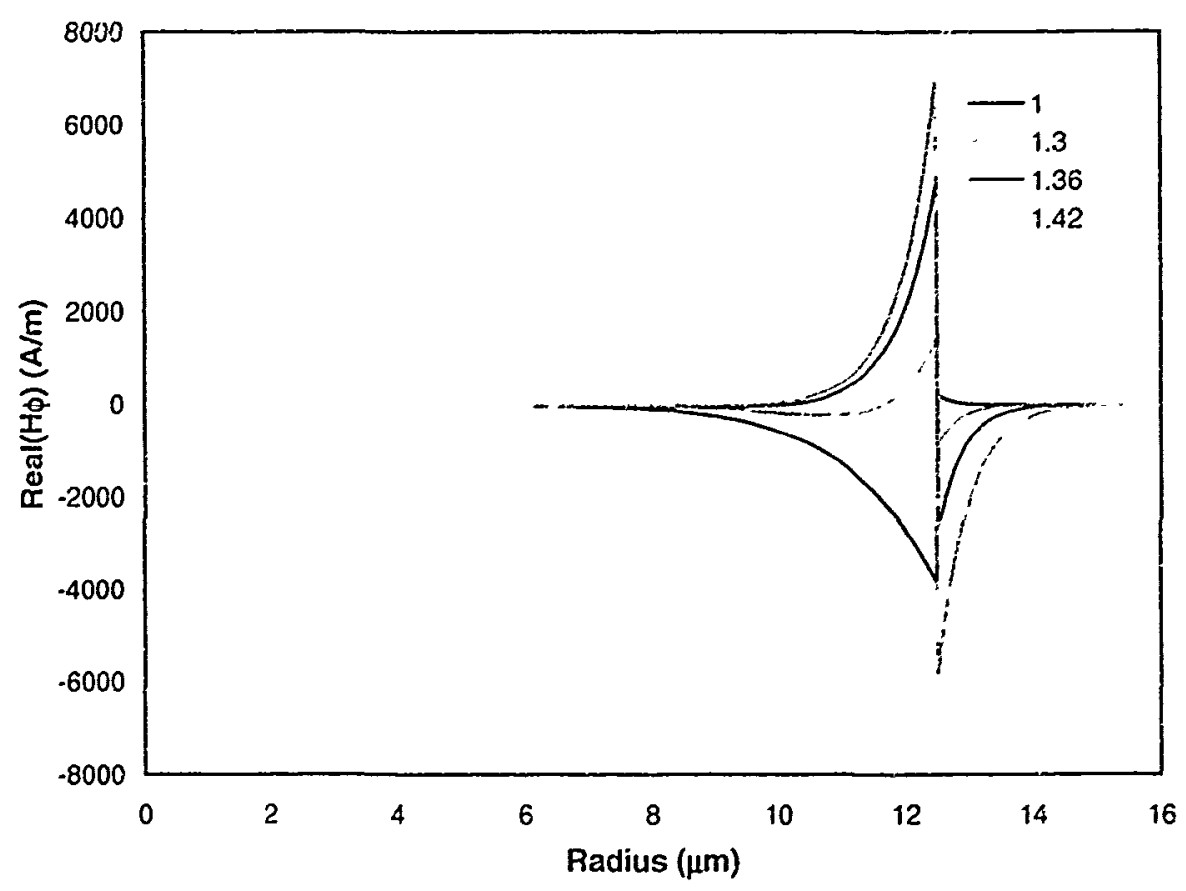

(c)

Figure 3.7: The normalized magnetic fields of plasmon modes with different SRI: (a) longitudinal component; (b) radial component, and (c) azimuthal component. 


\subsection{Numerical Simulation Issues}

When one or more layers are very thick and the modes are low order $\left(\mathrm{TE}_{0} / \mathrm{TM}_{0}\right)$ and strongly guided, then there is a well-known issue that the transfer matrix method may fail to obtain the correct field distributions in planar optical waveguides. The problem is the fields may already decay at the outer boundary of thick layer and the software program doesn't have enough effective digits to handle very small values (near zero). In that case, the solution is to remove the layers beyond the thick layer, because those removed layers won't affect the light propagation. Similarly for optical fibre, if the cladding layer is thick such as SMF-28 fibre, when calculating the core mode, the other cladding layer s.uch metal coating, thin dielectric overlay and surrounding media can be removed in calculations and the result won't be affected. Similar approach also can apply to multimode fibres. There is no problem for cladding modes as their fields always reach to the surrounding media when using the transfer matrix method.

The current programming software and languages such as Matlab, $\mathrm{C} / \mathrm{C}++$, Visual Basic and FORTRAN have a limitation for the minimum and maximum double/floating values to $\sim 10^{ \pm 30 x}$. In some specific case such as fibre with gold coating, the modifict Bessel functions will reach this maximum/minimum value when the fibre radius is $\sim 13$ $\mu \mathrm{m}$, therefore the other software tools are necded. Maple and Mathematica are the scientific calculation softw are that do not have the limitation for the maximum/minimum value. Here we use Matlab with the Maple toolbox for Matlab to do our simulation for the fibre modes in fibre with metal coatings. 


\subsection{Summary}

In this Chapter, an efficient and accurate numerical method to calculate the multilaye: fibre modes with lossless, lossy and gain media has been developed. The analytical inverse transfer matrix has been derived. By using the analytical inverse transfer matrix instead of numerical inverse matrix, the computing time is saved and accuracy is increased. More importantly, it makes analytical derivative of inverse matrix calculation possible, then the Cauchy's Integration Method can be used to solve the more complex step-index fibre with lossy and gain media especially for analyzing the plasmon-like modes in the fibre with metal coating. This provides a very useful tool the fast analysis of the fibre modes for our sensing applications and it can be further developed to an advanced step-index fibre mode solver for commercial CAD software.

Three different fibre structures of interest in our optical fibre sensing applications are calculated and compared with the literature. Good agreement is obtained. After this method is developed, it makes a large set of fibre mode calculations possible, which is especially important for TFBG sensors with a metal layer $[45,46]$. 


\section{Chapter 4}

\section{Mode Couplings in Optical Fibres with Lossless, Lossy}

\section{and Gain Media}

\subsection{Introduction}

The mode coupling in optical waveguides and fibres is very important. There are a lot of optical devices that are using the principle of mode couplings such as optical couplers, optical mode converters, grating based optical filters etc. Among those devices, a lot of them are optical long period gratings (LPG) and fibre Bragg gratings (FBG) based for both telecommunication and sensor applications. The theory for mode couplings in optical waveguides and DFB lasers was well developed [19] even before the FBG was invented in 1978 by Hill et al [3]. There are several methods that can be used to analyze the mode coupling in fibres, ameng them, the coupled mode theory is well developed and the one most often used to analyze LPGs and FBGs. In this Chapter, we focus on the fibre mode coupling between the core mode and the core and cladding modes due to sinusoidal index modulations in the fibre core layer with or without a small tilted angle. Comparing with previous work in the literature, what is new here can be listed as: (i) we use all vectorial modes to analyze mode couplings between the hybrid $\mathrm{HE}_{11}$ core mode to hybrid $\mathrm{HE}_{11}$ mode and $\mathrm{HE}_{\mathrm{vm}} / \mathrm{EH}_{\mathrm{vu}}$ cladding modes instead of using the approximate $\mathrm{LP}_{011}$ 
core mode to $L P_{v m}[47]$ or $L P_{01}$ to $\mathrm{HE}_{v \mathrm{~m}} / \mathrm{EH}_{\mathrm{vm}}$ cladding mode $[21,22]$; (ii) we solve for the mode couplings in a fibre with a thin metal coating for LPG [44] and TFBG applications [45]. This will give us a tool to analyze the TFBG in a fibre with gold coating in which some cladding modes show plasmon mode characteristics and a broad view to see what happens in TFBGs with a gold coating, which have shown a very high sensitivity for surrounding refractive index (SRI) changes and suitability for biosensor applications.

\subsection{The Electric Field Distributions in Multilayer Step-Index Fibre}

The mode couplings are fully dependent on the mode field distributions in the perturbation area. Here, for the single mode fibres we used the perturbation is located in the photosensitive core layer, so mode fields in the fibre core are the fundamental factor for designing optical fibre devices. For telecommunication applications, normally the fibre modes are analyzed in a two layer structure only since the core guided field does not extend to the outer boundary; three and more layers need to be analyzed in SMF fibre (core, cladding and surrounding medium) for index, strain and temperature sensing or coated SMF fibre with four laycrs (core, cladding, dielectric (metal) overli2y and surrounding medium) for cnhanced index sensing and cven more layers for fibres with gold overlay for Dirs sensing in which a thin DNA layer is binding to the gold surface.

Fig. 4.1 shows electric the ficld distributions of SMF-28 fibre with different hybrid modes and the insets show fields in the core region where the couplings between the core and cladding modes happen. From the Fig. 4.1, we cal see that the $E_{z}$ is at least one order of magnitude less that the transverse fields $E_{r}$ and $E_{\phi}$ especially for the core mode $H E_{i 1}$. 


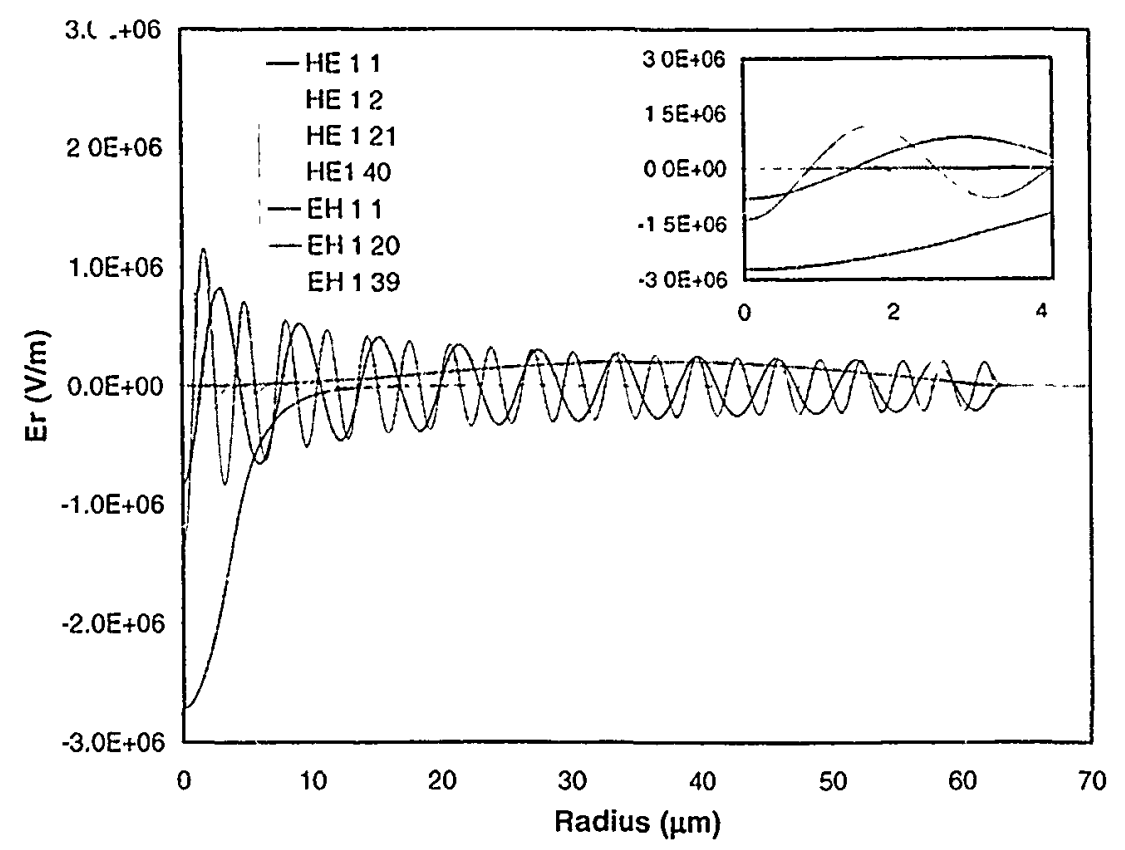

(a)

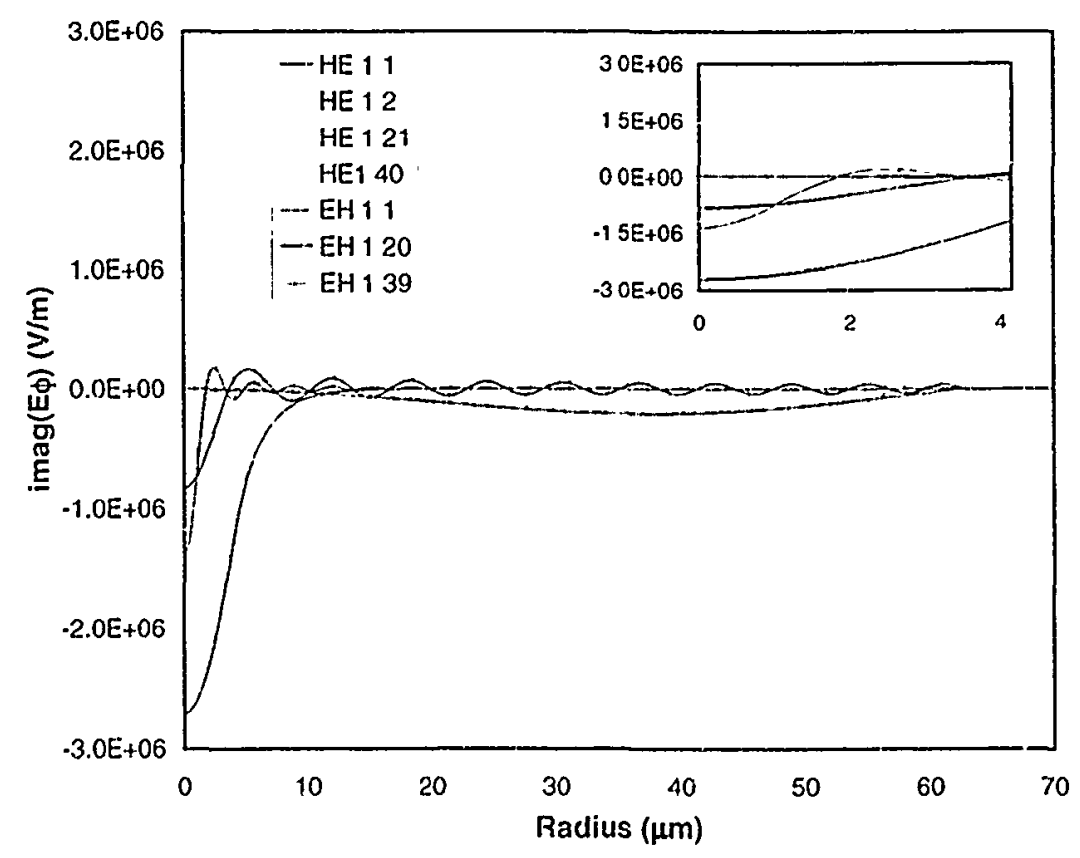

(b) 


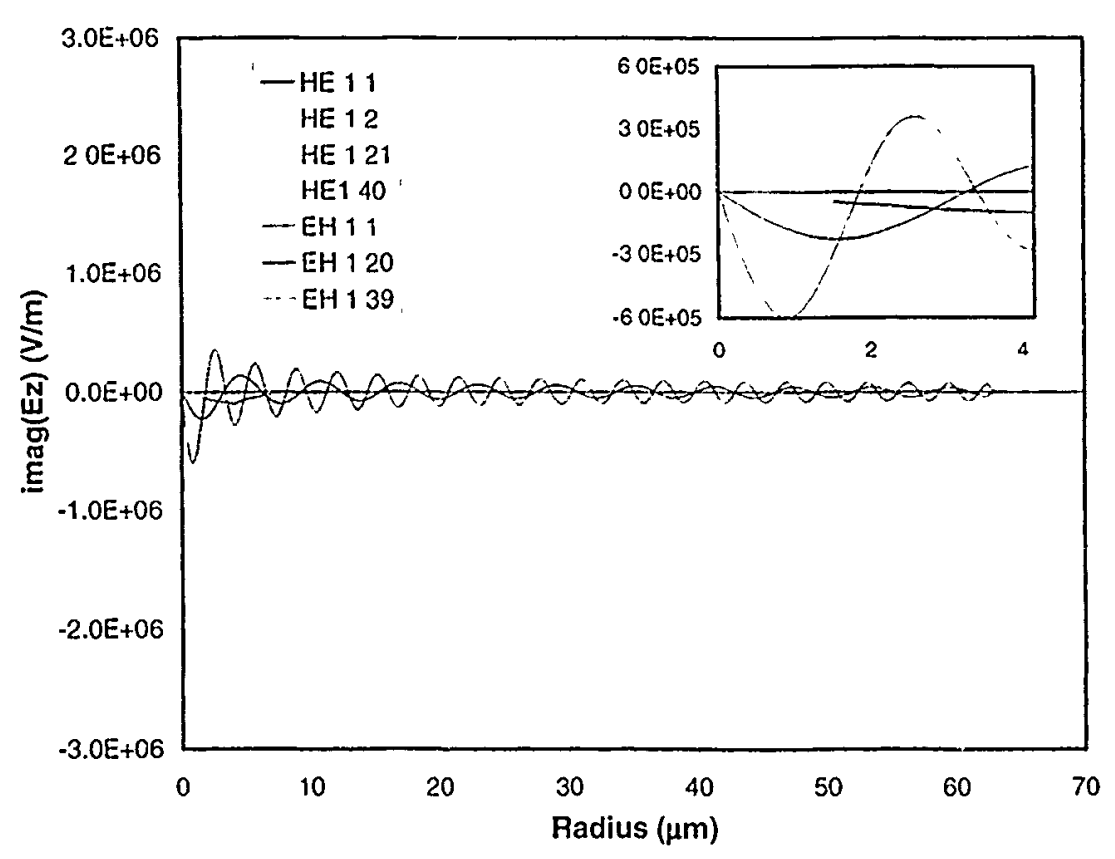

(c)

Figure 4.1: Electric field distributions in SMF-28 fibre.

\subsection{Cladding Mode Couplings with Core Mode}

In single mode fibres, the mode couplings occur between core and cladding modes with gratings such as FBGs and LPGs in the photosensitive core region. In most cases the core mode can be approximated using the $\mathrm{LP}_{01}$ linear polarization mode $[20]$ as standard SMFs are weakly guided. Even by writing strong gratings in the fibre core, the average refractive index of the core layer can be increased up to $10^{-2}$, the weakly guided conditions are still satisfied. We use the hybrid mode $\mathrm{HE}_{11}$ here, not due to the big core index increase when we write tilted FBG, but because the full vectorial mode makes the coupling mode calculations more accurate, especially in the case of plasmon mode coupling, in special fibres that are much thinner than in standard SMF fibres, where the 
fields of the core mode can realch the boundary between the silica and the metal and where the two layer $\mathrm{LP}_{01}$ mode approximation is no long sati .fied.

There are several methods that can be used to calculate the mode couplings in optical waveguides and fibres. Among them the coupled mode theory is well developed and widely used in analyzing mode couplings in fibres with FBGs and LPGs. The coupled mode analysis is based on perturbation theory and it can be derived from Maxweli's equations.

\subsubsection{Perturbation Theory $[35,48]$}

Mode fields without perturbations can be written as:

$$
\begin{aligned}
& \nabla \times \overrightarrow{\mathrm{E}}_{0}=-j \omega \mu \overrightarrow{\mathrm{H}}_{0} \\
& \nabla \times \overrightarrow{\mathrm{H}}_{0}=j \omega \varepsilon_{0} \varepsilon_{,}(r, \phi) \overrightarrow{\mathrm{E}}_{0}
\end{aligned}
$$

and mode fields with the dielectric perturbation $\Delta \varepsilon_{r}$ can be written as [48]:

$$
\begin{aligned}
& \nabla \times \overline{\mathrm{E}}=-\jmath \jmath \mu \overline{\mathrm{H}} \\
& \nabla \times \ddot{\mathrm{H}}=j \omega \varepsilon_{\mathrm{G}}\left[\varepsilon_{r}(r, \phi)+\Delta \varepsilon_{,}(r, \phi, z)\right] \overline{\mathrm{E}}
\end{aligned}
$$

Ficlds for forward propagating mode $v$ can be written as:

$$
\begin{aligned}
& \overrightarrow{\mathrm{E}}_{0}(r, \phi, z)=\left[\vec{e}_{t}(r, \phi)+\hat{z} c_{z}(r, \phi)\right] e^{-j \beta_{1} z} \\
& \overrightarrow{\mathrm{H}}_{0}(r, \phi, z)=\left[\vec{h}_{t}(r, \phi)+\hat{z} h_{z}(r, \phi)\right] e^{-J \beta_{r} z}
\end{aligned}
$$

and fields with perturbation can be written as a sum of all possible excited modes as [48]: 


$$
\begin{aligned}
& \overrightarrow{\mathrm{E}}(r, \phi, z)=\sum_{\mu} A_{\mu t}\left[\vec{e}_{\mu t}(r, \phi)+\hat{z} \frac{\varepsilon_{r}}{\varepsilon_{1}+\Delta \varepsilon_{r}(r, \phi, z)} e_{\mu z}(r, \phi)\right] e^{\mu \beta_{\mu \nu} z} \\
& +\sum_{\mu} B_{\mu}\left[\bar{e}_{\mu l}(r, \phi)-\hat{z} \frac{\varepsilon_{r}}{\varepsilon_{r}+\Delta \varepsilon_{r}(r, \phi, z)} e_{\mu z}(r, \phi)\right] e^{\dagger J \beta_{\mu}=} \\
& \overline{\mathrm{H}}(r, \phi, z)=\sum_{\mu} A_{\mu}\left[\vec{h}_{\mu t}(r, \phi)+\hat{z} \frac{\varepsilon_{r}}{\varepsilon_{r}+\Delta \varepsilon_{,}(r, \phi, z)} h_{\mu z}(r, \phi)\right] e^{-J \beta_{\mu} z} \\
& +\sum_{\mu} B_{\mu}\left[\vec{h}_{\mu r}(r, \phi)-\hat{z} \frac{\varepsilon_{r}}{\varepsilon_{r}+\Delta \varepsilon_{r}(r, \phi, z)} h_{\mu z}(r, \phi)\right] e^{+J \beta_{\mu^{2}}}
\end{aligned}
$$

Here we change ficlds from rectangular coordinates to cylindrical coordinates and backward propagating field terms are added. From Eqs. (4.1) and (4.2), we have [48]

$$
\nabla \cdot\left(\overrightarrow{\mathrm{E}}_{0}^{*} \times \overrightarrow{\mathrm{H}}+\overrightarrow{\mathrm{E}} \times \overrightarrow{\mathrm{H}}_{0}^{*}\right)=-j \omega \varepsilon_{0} \Delta \varepsilon_{r}(r, \phi, z) \overrightarrow{\mathrm{E}}_{0}^{*} \cdot \overrightarrow{\mathrm{E}}
$$

Substitutc Eqs. (4.3) and (4.4) into Eq. (4.5) and perform an area integral on both sides, the general coupled mode equations can be derived as [5]:

$$
\begin{aligned}
& \frac{d A_{\mu}}{d z}=-j \sum_{v} A_{v}\left(K_{v \mu}^{t}+K_{v \mu}^{i}\right) \exp \left[j\left(\beta_{\mu}+\beta_{v}\right) z\right]-j \sum_{v} B_{v}\left(K_{v \mu}^{t}-K_{v \mu}^{z}\right) \exp \left[j\left(\beta_{\mu}-\beta_{v}\right) z\right] \\
& \frac{d B_{\mu}}{d z}=j \sum_{v} A_{v}\left(K_{v \mu}^{t}+K_{v \mu}^{i}\right) \exp \left[-j\left(\beta_{\mu}+\beta_{v}\right) z\right]+j \sum_{v} B_{v}\left(K_{v \mu}^{t}-K_{v \mu}^{z}\right) \exp \left[-j\left(\beta_{\mu}-\beta_{v}\right) z\right]
\end{aligned}
$$

Where $K_{v y}^{\prime}$ and $K_{v y}^{:}$are the transverse coupling coefficient and longitudinal coefficient respectively, $A_{\mu}$ and $B_{\mu}$ are ficl. 1 amplitudes for forward and backward propagation of mode $\mu$.

$$
\begin{aligned}
& K_{v \mu h}^{t}(z)=\left(\omega \varepsilon_{0} / 4\right) \int_{1}^{2 \pi} d \phi \int_{0}^{\omega} r d r \Delta \varepsilon_{,}(r, \phi, z) \vec{e}_{v t}(r, \phi) \cdot \vec{e}_{\mu t}^{*}(r, \phi) \\
& K_{v i l}^{z}(z)=\left(\omega \mathcal{E}_{0} / 4\right) \int_{0}^{2 \pi} d \phi \int_{0}^{\infty} r d r \frac{\Delta \mathcal{E}_{r}(r, \phi, z) \varepsilon_{1}}{\varepsilon_{,}+\Delta \varepsilon_{r}(r, \phi, z)} e_{r_{i}}(r, \phi) \mathcal{k}_{\mu i}^{*}(r, \phi)
\end{aligned}
$$


From section 4.2, we know that the longitudinal electric field is at least one order of magnitude less than the transverse field, especially for the core mode, so the $K_{\mathrm{v} \mu}^{*}$ is at least two orders of magnitude less than the $K_{\mathrm{v} \mu}^{\prime}$. Therefore in the mode coupling calculations, the $K_{v y}^{z}$ is negligible. The Eq. (4.6) can be simplified as:

$$
\begin{aligned}
& \frac{d A_{\mu}}{d z}=-j \sum_{v} A_{v} K_{v \mu}^{\prime} \exp \left[j\left(\beta_{\mu}+\beta_{v}\right) z\right]-j \sum_{v} B_{v} K_{v \mu} \exp \left[j\left(\beta_{\mu}-\beta_{v}\right) z\right] \\
& \frac{d B_{\mu}}{d z}=j \sum_{v} A_{v} K_{v \mu}^{t} \exp \left[-j\left(\beta_{\mu}+\beta_{v}\right) z\right]+j \sum_{v} B_{v} K_{v / \mu}^{t} \exp \left[-j\left(\beta_{\mu}-\beta_{v}\right) z\right]
\end{aligned}
$$

\subsubsection{Core and Cladding Mode Coupling Coefficients in Fibres with FBG}

When a FBG or TFBG is induced in an optical fibre with UV light, normally only the photosensitive core refractive index is changed. The dielectric perturbation in the fibre can be written as

$$
\Delta \varepsilon_{r}(x, y, z, \theta)=2 n_{1} \Delta n_{\mathrm{i}}(x, y, z, \theta)
$$

where $\theta$ is a tilt angle. For FBG the tilt angle $\theta$ equals zero, the core layer refractive index perturbation can be written as

$$
\Delta n_{1}(x, y, z)=n_{1} \sigma(z)[1+M \cos (2 K z)]
$$

Where $n_{1}$ is unperturbated core index, $\sigma(z)$ is a slowly varying envelope, $M$ is the refractive index modulation amplitude induced in the grating, or the grating visibility, $2 K=2 \pi / \Lambda$ is the wave number of the grating and $/$ is the grating period. Substituting the Eq. (4.10) into Eq. (4.7a), we can represent the coupling coeflicient as [21]

$$
K_{v \mu \mu}^{t}(z)=\kappa_{v \mu}\left[1+M \cos \left(2 K_{z}\right)\right]
$$


and the coupling constant

$$
\kappa_{v \mu l}(z)=\frac{\omega \varepsilon_{0} n_{1}^{2} \sigma(z)}{2} \int_{0}^{2 \pi} d \phi \int_{0}^{r_{1}} \vec{e}_{v}(r, \phi) \cdot \vec{e}_{\mu l}^{\alpha}(r, \phi) r d r
$$

$\kappa_{\mathrm{v} y}$ is defined as the coupling constant even through it can have a slowly varying $z$ dependence, but it will be constant if the grating is uniform.

From the Chapter 3, transverse electric ficlds in the fibre core can be written as:

$$
\begin{aligned}
& e_{r}=\left[-\frac{\beta u_{1}}{2 \omega \varepsilon_{1}} A_{1}\left(J_{v-1}\left(u_{1} r\right)-J_{v+1}\left(u_{1} r\right)\right)+\frac{u_{1}}{2} C_{1}\left(J_{v-1}\left(u_{1} r\right)+J_{v+1}\left(u_{1} r\right)\right)\right] \exp (j v \phi) \\
& e_{\phi}=j\left[-\frac{\beta u_{1}}{2 \omega \varepsilon_{1}} A_{1}\left(J_{v-1}\left(u_{1} r\right)+J_{v^{\prime}+1}\left(u_{1} r\right)\right)+\frac{u_{1}}{2} C_{1}\left(J_{v-1}\left(u_{1} r\right)-J_{v+1}\left(u_{1} r\right)\right)\right] \exp (j v \phi)
\end{aligned}
$$

Here $A_{1}$ and $C_{1}$ are normalized field constants for core and cladding modes by specifying that each mode carry a power of $1 \mathrm{~W}$.

$$
P=1 / 2 \operatorname{Re}\left[\int_{0}^{2 \pi} d \phi \int_{0}^{\infty}\left(e_{r} h_{\phi}^{*}-e_{\phi} h_{r}^{*}\right) r d r\right]=1 \mathrm{~W}
$$

The integral is different for lossless and loss/gain fibre mode. The solution to the integral can be written in a closed form, but the expression is very long. The results of integral calculation are provided in Appendix A. In the remainder of this Chapter, each mode field coefficients are first normalized to $1 \mathrm{~W}$ before performing the mode coupling calculation.

For simplifying the mode coupling constant calculation, Eq. (4.13) can be written as:

$$
\begin{aligned}
& \mathcal{c}_{r}=\left(\tilde{\Lambda}_{1} J_{v-i}\left(u_{1} r\right)+\tilde{B}_{1} J_{v+1}\left(u_{1} r\right)\right) \exp (j v \phi) \\
& c_{s}=j\left(\tilde{A}_{1} J_{v-1}\left(u_{1} r\right)-\tilde{B}_{1} J_{v+1}\left(u_{1} r\right)\right) \exp (j v \phi)
\end{aligned}
$$


where

$$
\begin{aligned}
& \tilde{A}_{1}=-\frac{\beta u_{1}}{2 \omega \varepsilon_{1}} A_{1}+\frac{u_{1}}{2} C_{1} \\
& \tilde{B}_{1}=\frac{\beta u_{1}}{2 \omega \varepsilon_{1}} A_{1}+\frac{u_{1}}{2} C_{1}
\end{aligned}
$$

Substituting Eq. (4.15) into Eq. (4.12) with $v=1$, we obtain the core mode to core mode coupling constant

$$
\kappa_{11-11}(z)=\pi \omega \varepsilon_{0} n_{1}^{2} \sigma(z) \int_{0}^{r_{1}}\left(\left|\tilde{A}_{1}\right|^{2}\left|J_{0}\left(u_{1} r\right)\right|^{2}+\left|\tilde{B}_{1}\right|^{2}\left|J_{2}\left(u_{1} r\right)\right|^{2}\right) r d r
$$

So the core-core mode coupling constant will be real even through the $n_{\text {cff }}$ could be complex if $n_{l}$ is real. To solve Eq. (4.17), if $n_{\text {eff }}$ is real, then we have

$$
\kappa_{11-11}(z)=\pi \omega \varepsilon_{0} n_{1}^{2} \sigma(z) r_{1}^{2}\left[\left(J_{0}^{2}\left(u_{1} r_{1}\right)+J_{1}^{2}\left(u_{1} r_{1}\right)\right) \dot{A}_{1}^{2}+\left(J_{2}^{2}\left(u_{1} r_{1}\right)-J_{1}\left(u_{1} r_{1}\right) J_{3}\left(u_{1} r_{1}\right)\right) \tilde{B}_{1}^{2}\right]
$$

and if $n_{e f f}$ is complex, for conjugate of Bessel function, we have $J_{0}^{*}\left(u_{1} r\right)=J_{0}\left(u_{1}^{*} r\right)$ and $J_{2}^{*}\left(u_{1} r\right)=J_{2}\left(u_{1}^{*} r\right)$, then

$$
\begin{aligned}
\kappa_{11-11}(z) & =2 \pi \omega \varepsilon_{0} n_{1}^{2} \sigma(z) r_{1} /\left(u_{1}^{2}-\left(u_{1}^{*}\right)^{2}\right) \\
& \cdot\left\{\left[u_{1} J_{0}\left(u_{1}^{*} r_{1}\right) J_{1}\left(u_{1} r_{1}\right)-u_{1}^{*} J_{0}\left(u_{1} r_{1}\right) J_{1}\left(u_{1}^{*} r_{1}\right)\right]\left|\tilde{A}_{1}\right|^{2}\right. \\
+ & {\left.\left[u_{1}^{*} J_{1}\left(u_{1}^{*} r_{1}\right) J_{2}\left(u_{1} r_{1}\right)-u_{1} J_{1}\left(u_{1} r_{1}\right) J_{2}\left(u_{1}^{*} r_{1}\right)\right]\left|\tilde{B}_{1}\right|^{2}\right\} }
\end{aligned}
$$

The coupling constant for core mode to cladding mode coupling can be derived by substituting Eq. (4.15) into Eq. (4.12)

$$
\kappa_{v-11}(z)=\frac{\omega \varepsilon_{v,} n_{1}^{2} \sigma(z)}{2} \int_{0}^{2 \pi} d \phi \int_{j}^{\mathrm{t}_{1}}\left(e_{v r} e_{1 r}^{*}+e_{v \phi} e_{1 \phi}^{*}\right) r d r
$$

For any cladding mode, (any $v$ ), the azimuthal integral in Eq. (4.20) becomes 


$$
\int_{0}^{2 \pi} \exp [j(1-v) \phi] d \phi=2 \pi \delta_{1 v}
$$

where $\delta_{l y}$ is the Kronecker delta function

$$
\delta_{1 v}= \begin{cases}1, & \text { if } v=1 \\ 0, & \text { if } v \neq 1\end{cases}
$$

Therefore, only those cladding modes which have $v=1$ have the non-zero coupling constant with the $\mathrm{HE}_{11}$ core mode. Performing the integral of Eq. (4.20), the coupling constant of core mode $\mathrm{HE}_{11}$ to $\mathrm{HE}_{1 \mathrm{~m}} / \mathrm{EH}_{1 \mathrm{~m}}$ cladding modes can be obtained as

$$
\begin{aligned}
\kappa_{1 m-11}(z)= & 2 \pi \omega \varepsilon_{0} n_{1}^{2} \sigma(z) r_{1} /\left(u_{1 m}^{2}-\left(u_{1}^{*}\right)^{2}\right) \\
& \cdot\left\{\left[u_{1 m} J_{0}\left(u_{1}^{*} r_{1}\right) J_{1}\left(u_{1 m} r_{1}\right)-u_{1}^{*} J_{0}\left(u_{1 m} r_{1}\right) J_{1}\left(u_{1}^{*} r_{1}\right)\right] \tilde{A}_{1 m} \tilde{A}_{1}^{*}\right. \\
+ & {\left.\left[u_{1}^{*} J_{1}\left(u_{1}^{*} r_{1}\right) J_{2}\left(u_{1 m} r_{1}\right)-u_{1 m} J_{1}\left(u_{1 m} r_{1}\right) J_{2}\left(u_{1}^{*} r_{1}\right)\right] \tilde{B}_{1 m} \tilde{B}_{1}^{*}\right\} }
\end{aligned}
$$

Eq. (4.23) is a general solution of the coupling constant of cladding modes to core mode coupling for lossless, lossy and gain fibre.

\subsubsection{Core and Cladding Mode Coupling Coefficients in Fibres with TFBG}

When a TFBG is induced in an optical fibre with UV light with a tilt angle $\theta$, as shown in Fig. 4.2, there are two possible tilted gratings including a grating whose planes are tilted about the $\mathrm{y}$ axis by an angle $\theta$ in the clockwise direction from the $\mathrm{x}$ axis (an $\mathrm{x}$-tilted grating) and a grating whose planes are tilted about the $\mathrm{x}$ axis by an angle $\theta$ from the $\mathrm{y}$ axis (a y-tilted grating) [22], which are corresponding to two linear polarization states. The dielectric perturbation in the fibre can be written as 


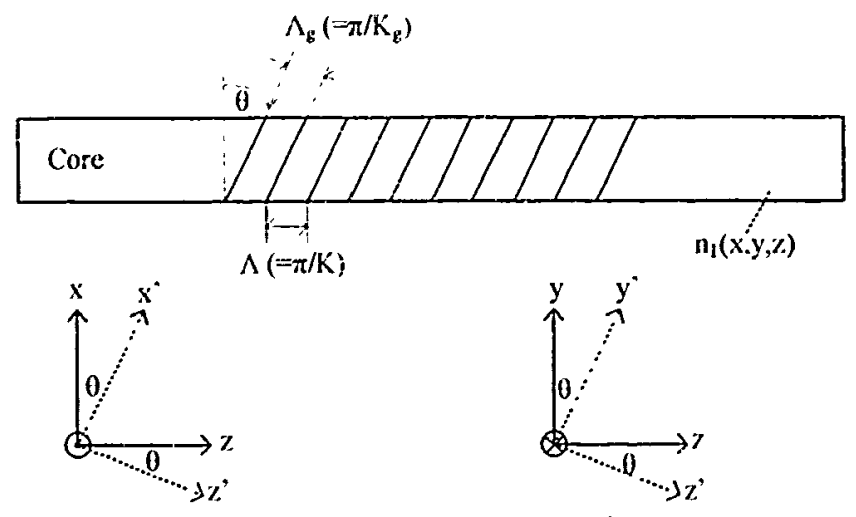

(a)

(b)

Figure 4.2: Configuration of a single-sided tilted grating: (a) for an $x$-tilted grating, (b) for a $y$-tilted grating.

$$
\Delta \varepsilon_{r}(x, y, z, \theta)=2 n_{1} \Delta n_{1}(x, y, z, \theta)
$$

and the core layer refractive index perturbation can be written as

$$
\Delta n_{1}(x, y, z)=n_{1} \sigma\left(z^{\prime}\right)\left[1+M \cos \left(2 K_{g^{\prime}} z^{\prime}\right)\right]
$$

where

$$
\begin{aligned}
& z^{\prime}=z \cos \theta-x \sin \theta, \text { for an } x \text {-tilied grating } \\
& z^{\prime}=z \cos \theta-y \sin \theta, \text { for a y-tilted grating }
\end{aligned}
$$

Where $n_{1}$ is unperiurbated core index, $\sigma\left(z^{\prime}\right)$ is a slowly varying envelope, $M$ is the refractive index modulation amplitude induced in the grating, or the grating visibility. The nominal wave number of the tilted grating, $2 K_{x}=2 \pi / \Lambda_{g}$, is related to the grating wave-vector component along the fibre axis $2 K=2 \pi / \Lambda$, where

$$
2 K=2 K_{k} \cos \theta
$$


Herc $\Lambda=\Lambda_{g} / \cos \theta$ is the grating period along the fibre axis and $\Lambda_{x}$ is the actual grating period. Substituting Eqs. (4.25) and (4.26) into Eq. (4.7a), the coupling coefficient $K_{v \mu}^{\prime}$ can be expressed as a summation of three distinct coupling coefficient components [22]

$$
K_{v \mu}^{t}(z)=g_{v \mu}^{+} \exp \left(j 2 K_{g} z \cos \theta\right)+g_{v \mu}^{-} \exp \left(-j 2 K_{g} z \cos \theta\right)+f_{v \mu}
$$

Where

$$
g_{v \mu}^{ \pm}=\frac{\omega}{4} \varepsilon_{0} n_{1}^{2} \sigma(z) M \int_{0}^{2 \pi} d \phi \int_{0}^{1} \exp \left(\mp j 2 K_{g} z \cos \phi \sin \theta\right) \vec{e}_{v t}(r, \phi) \cdot \vec{e}_{\mu t}^{*}(r, \phi) r d r
$$

for an $x$-tilted grating

$$
g_{v \mu}^{ \pm}=\frac{\omega}{4} \varepsilon_{0} n_{1}^{2} \sigma(z) M \int_{0}^{2 \pi} d \phi \int_{0}^{T_{1}} \exp \left(\mp j 2 K_{x} z \sin \phi \sin \theta\right) \vec{e}_{v i}(r, \phi) \cdot \bar{e}_{\mu t}^{*}(r, \phi) r d r
$$

for a y-tilted grating, and

$$
f_{v \mu}=\frac{\omega}{2} \varepsilon_{0} n_{1}^{2} \sigma(z) \int_{0}^{2 \pi} d \phi \int_{0}^{1} \vec{e}_{v i}(r, \phi) \cdot \bar{e}_{\mu t}^{*}(r, \phi) r d r
$$

Here the $f_{v \mu}$ is the same as $\kappa_{v \mu}$ in section 4.3.2, substituting the Eq. (4.14) into Eq. (4.29) and using Bessel function relations [38], for the coupling between the $\mathrm{HE}_{11}$ to cladding mode $\mathrm{HE}_{\mathrm{v}} / \mathrm{EH}_{\mathrm{v}}$. we have

$$
\begin{aligned}
g_{v-11}^{ \pm} & =(\mp j)^{r-1} \pi \omega \varepsilon_{0} n_{1}^{2} \sigma(z) M / 2 \\
& \cdot \int_{1}^{r_{1}}\left[\tilde{A}_{v} \tilde{A}_{1}^{*} J_{v-1}(u r) J_{0}^{*}\left(u u_{1} r\right)+\tilde{B}_{v} \tilde{B}_{1}^{*} J_{v^{+}+1}(u r) J_{2}^{*}\left(u_{1} r\right)\right] J_{v-1}\left(2 K_{s} r \sin \theta\right) r d r
\end{aligned}
$$

for an $\mathrm{x}$-tilted grating

$$
\begin{aligned}
g_{v-11}^{\prime}= & (\mp 1)^{v-1} \pi \omega \varepsilon_{0} n_{1}^{2} \sigma(z) M / 2 \\
& \int_{1}^{r}\left[\tilde{A}_{v} \tilde{A}_{1}^{*} J_{v-1}(u r) J_{0}^{*}\left(u_{1} r\right)+\tilde{B}_{v} \tilde{B}_{1}^{*} J_{v+1}(u r) J_{2}^{*}\left(u_{1} r\right)\right] J_{v-1}\left(2 K_{k} r \sin \theta\right) r d r
\end{aligned}
$$

for a $y$-tilted grating 


$$
f_{v-11}=\pi \omega \varepsilon_{0} n_{1}^{2} \sigma(z) \int_{0}^{1}\left[\tilde{A}_{1}, \tilde{A}_{1}^{*} J_{v-1}(u r) J_{0}^{*}\left(u_{1} r\right)+\tilde{B}_{v} \tilde{B}_{1}^{*} J_{v+1}(u r) J_{2}^{*}\left(u_{1} r\right)\right] r d r
$$

For $f_{v-11}$, only those cladding modes which have $v=1$ have the non-zero coupling constant with the $\mathrm{HE}_{11}$ core mode, and they are the same as $\kappa_{11-11}$ for core to core mode coupling and $\kappa_{1 m-11}$ for cladding modes to core mode couplings of Eqs (4.17-4.19). Let

$$
\begin{aligned}
g_{v-11} & =\pi \omega \varepsilon_{0} n_{1}^{2} \sigma(z) M / 2 \\
& \cdot \int_{0}^{\tau_{1}}\left[\tilde{A}_{v} \tilde{A}_{1}^{*} J_{v-1}(u r) J_{0}^{*}\left(u u_{1} r\right)+\tilde{B}_{v} \tilde{B}_{1}^{*} J_{v+1}(u r) J_{2}^{*}\left(u u_{1} r\right)\right] J_{v-1}\left(2 K_{z} r \sin \theta\right) r d r
\end{aligned}
$$

For an $\mathrm{x}$-tilted grating, the coupling coefficient

$$
K_{v-11}(z)=K_{1 m-11}+ \begin{cases}2 g_{v-11} \cos \left(2 K_{z} z \cos \theta\right) \exp (\mathrm{jk} \pi) & \text { for } v=1,3, \cdots, 2 \mathrm{k}+1 \\ 2 g_{v-11} \sin \left(2 K_{g} z \cos \theta\right) & \text { for } v=2,4, \cdots, 2 \mathrm{k}+2\end{cases}
$$

For a y-tilted grating, the coupling coefficient

$$
K_{v-11}(z)=\kappa_{1 m-11}+ \begin{cases}2 g_{v-11} \cos \left(2 K_{g} z \cos \theta\right) & \text { for } v=1,3, \cdots, 2 \mathrm{k}+1 \\ 2 g_{v-11} \sin \left(2 K_{g} z \cos \theta\right) \exp (-\mathrm{j} \pi / 2) & \text { for } v=2,4, \cdots, 2 \mathrm{k}+2\end{cases}
$$

Where $k=0,1,2 \cdots$, from Eqs. (4.32) and (4.33), we can sce that the $\mathrm{x}$-tilted grating and the $y$-tilted grating have the same DC coupling constant $\kappa_{1 m-11}$ and same amplitude for AC coupling constant $2 g_{v-11}$ but a phase difference of $\pi$ and $\pi / 2$ for the odd and even azimuthal number $v$, respectively. 


\subsection{Numerical Results}

\subsubsection{Coupling Constants in SMF with FBG}

\section{a) Case 1}

Fig. 4.3 shows the coupling constant $\kappa_{1 m-11}$ divided by $\sigma(z)$ for the SMF fibre of reference [21] using Eq. (4.23) for $v=1$, where $\sigma(z)$ is a slowly varying envelupe. The fibre is described by the following parameters: $n_{1}=1.458, n_{2}=1.45$, $n_{3}=1.0, r_{1}=2.625 \mu \mathrm{m}, r_{2}=62.5 \mu \mathrm{m}$ and $\lambda=1.55 \mu \mathrm{m}[21]$. There are in total 168 guided modes for $v=1$ including $65 \mathrm{HE}_{\mathrm{lm}}$ modes and $63 \mathrm{EH}_{1 \mathrm{~m}}$ modes. From Fig. 4.3, it can be seen that the cor lings between the lowest-order $\mathrm{EH}_{\mathrm{Im}}$ cladding modes and the core mode are very weak compared to the couplings between the lowest-order $\mathrm{HE}_{1 \mathrm{~m}}$ cladding modes and the corc mode. This can be explained by the mode classifications (field distributions) for $\mathrm{HE}$ and $\mathrm{EH}$ modes, obviously the $\mathrm{HE}_{1 \mathrm{~m}}$ mode will have larger coupling with $\mathrm{HE}_{11}$ mode than the $\mathrm{EH}_{1 \mathrm{~m}}$ mode with $\mathrm{HE}_{11}$ mode. Huwever, for cladding modes of order $m \geq 20$, the $\mathrm{EH}_{1 \mathrm{~m}}$ and $\mathrm{HE}_{1 \mathrm{~m}}$ modes have coupling strengths comparable to one another, this means that $\mathrm{HE}_{1 \mathrm{~m}}$ and $\mathrm{EH}_{1 \mathrm{~m}}$ modes with higher $m$ showing more similar ficld distributions in the fibre core region, it can be seen from Fig. 4.1. "The slowly varying oscillation in the coupling strength versus cladding mode number is analogous to the oscillation with respect to wavelength observed in coupling from the core mode to the continuum of radiation modes of the fibre. Qualitatively, this oscillation arises because as the cladding-mode order increases the mode fields exhibit more and more oscillations along the radial direction. As the number of oscillations increases within the fixed radial 
extent of the fibre, the cladding-mode fields begın to exhibit nulls in the fibre core. Each time a new null moves into the core, it is possible for the overlap integral in Eq. (4.23) to become zero, thus causing the zcros in the slowly varying envelope in Fig. 4.3 [21]". Comparing our result with the Fig. 6 of reference [21], the mode coupling has similar envelopes and nulls, but our coupling constant is about $38 \%$ higher than theirs. The difference is caused by using the $\mathrm{HE}_{11}$ core mode vs $L \mathrm{P}_{01}$ core mode. In LP $\mathrm{P}_{11}$ core mode approximation, also the normalized power is equal to $\mathrm{JW}$, but transverse electric fields for $E_{r}$ and $E_{\phi}$ only have one Besscl function term $J_{0}[4]$, and transverse electric fields for $\mathrm{HE}_{1 \mathrm{~m}} / \mathrm{EH}_{1 \mathrm{~m}}$ cladding modes have two terms $J_{0}$ and $J_{2}$. This might lead to the integral in Eq. (4.12) to generate the differences.

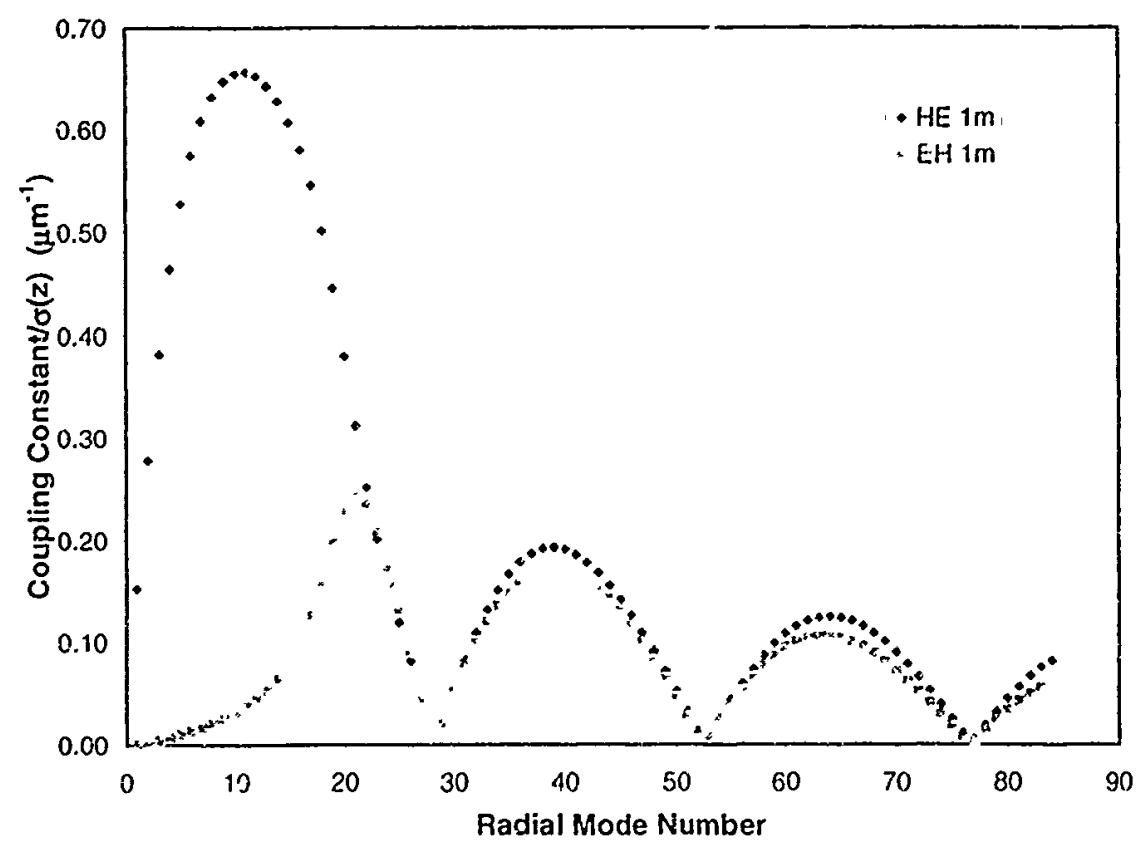

Figure 4.3: Coupling constant $\kappa_{1 m \| 1}$ divided by $\sigma(z)$ for the $168 v=1$ cladding modes in a SMF fibres. 


\section{b) Case 2}

Fig. 4.4 shows the coupling constant $\kappa_{1 m-11}$ divided by $\sigma(z)$ for the fibre that we used experimentally (SMF 28) using Eu! (4.23) for $v=1$. The fibre parameters: $n_{1}=1.4504$, $n_{2}=1.444024, n_{3}=1.0, r_{1}=4.15 \mu \mathrm{m}, r_{2}=62.5 \mu \mathrm{m}$ and $\lambda=1.55 \mu \mathrm{m}$. Comparing with Fig. 4.3, there are similar oscillations of envelope, but the first null of the envelope is $\mathrm{m}=17$ for $\mathrm{EH}_{1 \mathrm{~m}}$ and $\mathrm{m}=18$ for $\mathrm{HE}_{\mathrm{Im}}$ mode instcad of in the Fig. $4.3, \mathrm{~m}=28$ for $\mathrm{EH}_{\mathrm{Im}}$ and $m=29$ for $\mathrm{HE}_{\mathrm{im}}$ mode, this is due to the fibre core radius difference of two fibres. The SMF-28 has core radius of $4.15 \mu \mathrm{m}$ and the SMF fibre core radius is $2.625 \mu \mathrm{m}$. The larger core makes the cladding-mode fields of SMF 28 fibre exhibit the rull in the fibre core with small $m$.

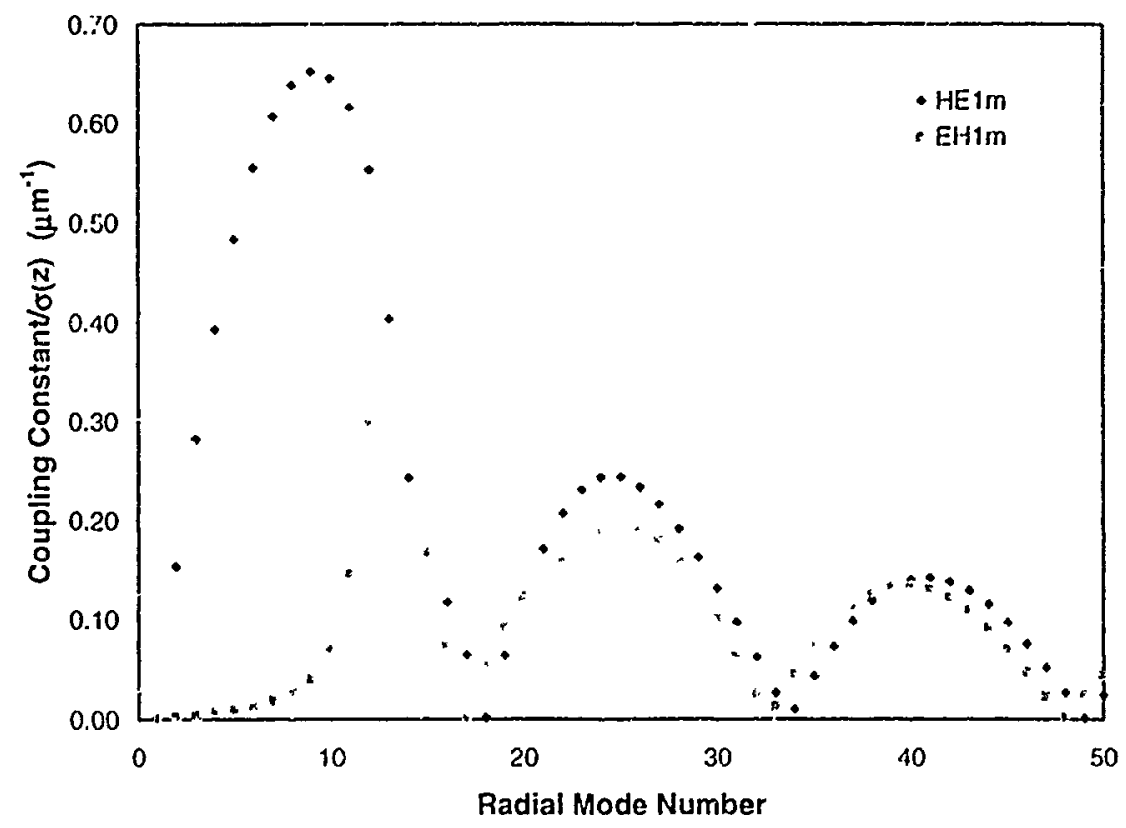

Figure 4.4: Coupling constant $\kappa_{|m-1|}$ divided by $\sigma(z)$ for $v=1$ cladding modes in the SMF28 fibre. 


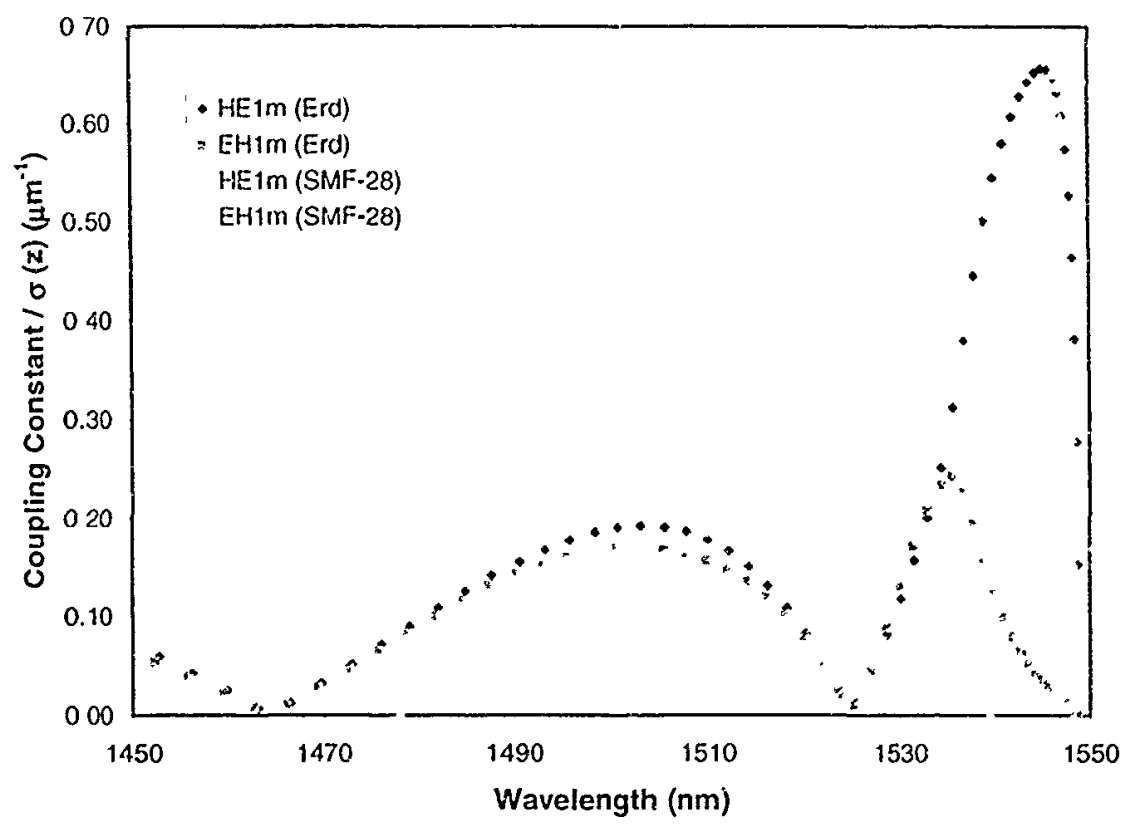

Figure 4.5: Comparing the coupling constant $\kappa_{\mathrm{l} m-1}$ divided by $\sigma(z)$ for $v=1$ cladding modes in a small core SMF fibre and SMF-28 fibre.

To directly compare mode couplings in these two fibres, we draw the coupling constants for thu fibres respect to the wavelength instead of mode number; the core mode (Bragg) resonance wavelength is $1550 \mathrm{~nm}$. From Fig. 4.5, we can see that the SMF-28 reaches the maximum coupling constant faster than the SMF with small core radius, and the maximum coupling constant values for these two fibres are very close even through they have totally different fibre parameters. The SMF 28 fibre has the first null of envelope around $1539.52 \mathrm{~nm}$ and SMF with small core radius has the first null around $1525.34 \mathrm{~nm}$. The maximum and null coupling constant values in different wavelength with different core radius could be useful for sensing applications: by changing the core radius we can have the strong or weak resonances at the wavelength we are interested for specific optical fibre sensors. 
Fig. 4.6 shows the transmission spectrum of one FBG written in SMF-28 fibre and the coupling constant $\kappa_{1 m-11}$ divided by $\sigma(z)$ for a SMF 28 fibre. From Fig. 4.6, we can see that simulation result is a good match to the resonance wavelength and the peak value of the cladding modes, even though we used the fibre parameter (core layer refractive index) of a TFBG instead of a FBG and the coupling constants simulated at $1550 \mathrm{~nm}$ are now used at $1560.32 \mathrm{~nm}$. That means we can directly simulate the coupling constants to predict the FBG resonances envelope (resonance strength) and without having to do a full simulation for the transmission spectrum of the FBG.

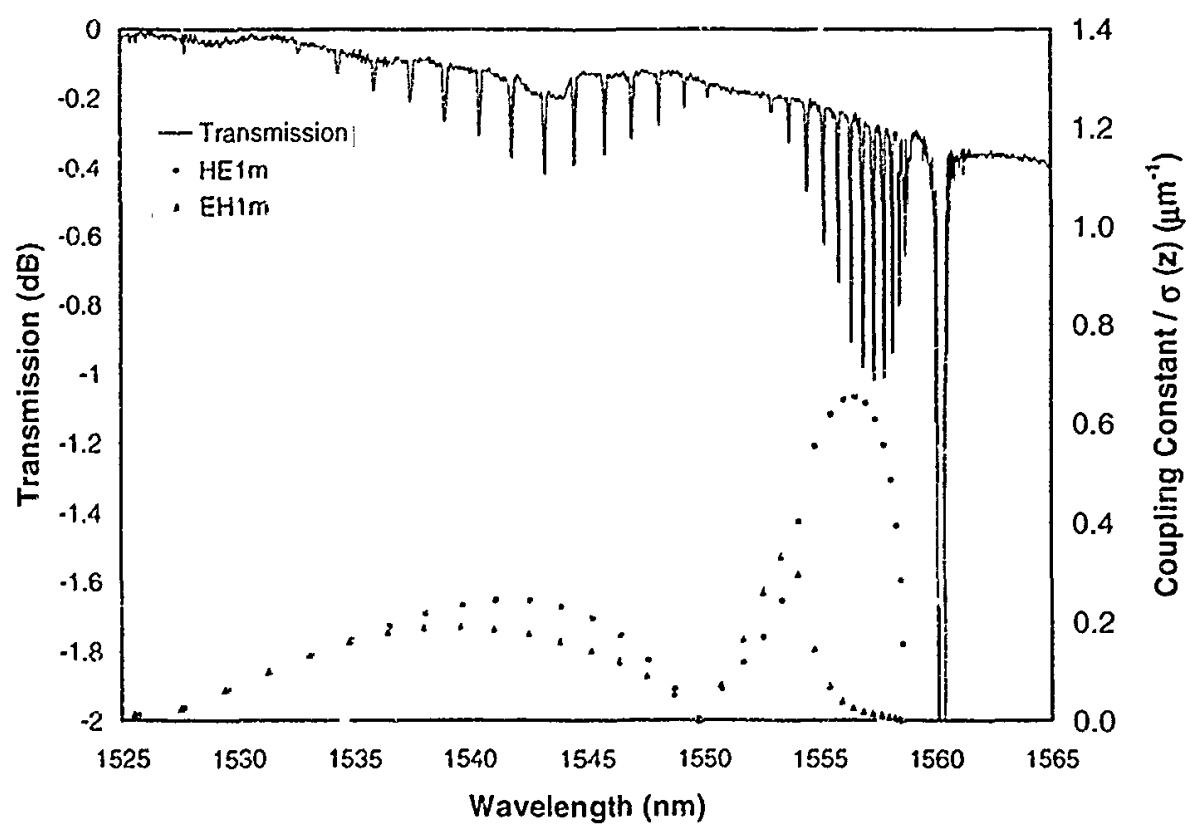

Figure 4.6: The transmission spectrum of a FBG in the SMF-28 fibre and simulated coupling constant $\kappa_{|m-1|}$ divided by $\sigma(z)$ for $v=1$ cladding modes in the SMF-28 fibre. 
c) Case 3

Fig. 4.7 shows the coupling constant $\kappa_{1 m-11}$ divided by $\sigma(z)$ for a SMF 28 libre with a $20 \mathrm{~nm}$ gold coating. The fibre parameters are: $n_{1}=1.4504, n_{2}=1.444024$, $n_{3}=0.55-j 11.5, n_{+}=1.0, r_{1}=4.15 \mu \mathrm{m}, \quad r_{2}=62.5 \mu \mathrm{m}, r_{3}=62.52 \mu \mathrm{m}(d=20 \mathrm{~nm})$, and $\lambda=1.55 \mu \mathrm{m}$. The cladding mudes in fibres with a thin . etal co:ting can sho: some plasmon mode characteristics: an extraurdinary sensitivity to surruanding refractive iridex ch. Iges as we discussed in Chapter 3, which attracts lot of interest recently for biosensor applications. Comparing with the SMF-28 without a gold coating (Fig. 4.4), now we can see the maxitum coupling constant is reduced by $27 \%$, and the coupling constant of $H E_{1 m}$ and $E H_{1: r}$ are close to each other when $m \geq 12$.

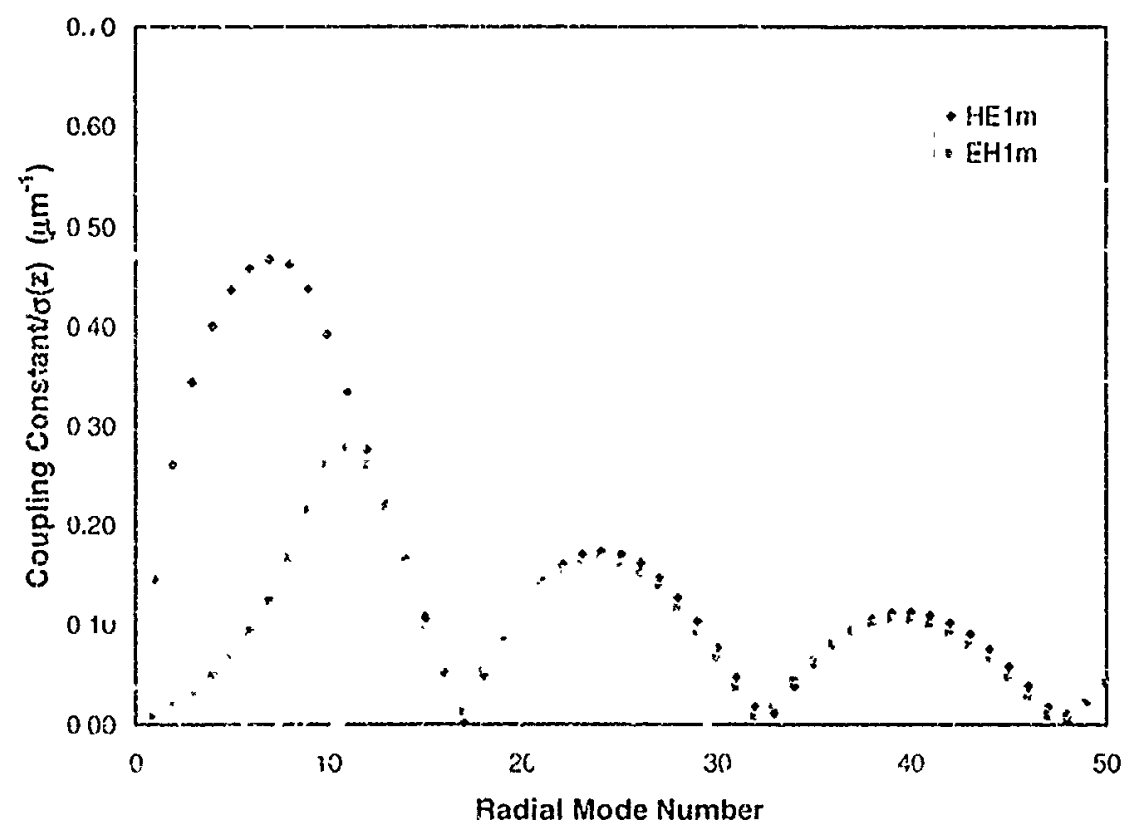

Figure 4.7: Compling constant $\kappa_{\mathrm{l} m \text { il }}$ di ided by $\sigma(z)$ for $\imath^{\prime}=1$ cladding modes in a SMF-28 fihre wit! $20 \mathrm{~mm}$ gold corting. 


\subsubsection{Coupling Constants in SMF with TFBG}

For the libre with TFBG, there is no analytical solution for coupling constant of Eq. (4.31). Therefore, numcrical integration for Eq. (4.31) is needed.

\section{a) Case 1}

Fig. 4.8 shows the AC coupling constant $2 g_{1^{\prime} m-1 /}$ divided by $\sigma(z)$ for a SMF fibre using $\mathrm{Eq} .(4.31)$ and assuming $\mathrm{M}=1$ with different azimuthal number $v$ and tilt angle $\theta$. The fibre is described by the following parameters: $n_{1}=1.458, n_{2}=1.45$, $n_{3}=1.0, r_{1}=2.5 \mu \mathrm{m}, r_{2}=62.5 \mu \mathrm{m}$ and $\lambda=1.55 \mu \mathrm{m}[22]$. There are in total 168 guided modes for $v=1$ including $65 \mathrm{HE}_{1 \mathrm{~m}}$ modes and $6.3 \mathrm{EH}_{1 \mathrm{~m}}$ modes as shown in Fig. 4.8 (a). The coupling constants are similar with Fig. 4.4, because the only difference for these two fibres is the $0.125 \mu \mathrm{m}$ core radius difference. The maximum coupling constant is at radial mode number $m=9$ of $\mathrm{HE}$ mode and the first null is the $\mathrm{EH}$ mode for $\mathrm{m}=30$. Fig. 4.8(b) shows tis $\Lambda \mathrm{C}$ coupling constant of th. libre with $v=2$ and $\theta=5^{\circ}$. From Fig. 4.8(b), it can be secn that the maximum coupling constant is smaller than the un-tilted one and shift to the higher radial mode number $m=19$ of $\mathrm{HE}_{2 m}$ mode, the lirst null in the envelope is shifted to the high radial mode number as well and at $m=45$ for both ${ }_{11 E_{2 m}}$ and $\mathrm{EH}_{2 \mathrm{~m}}$ modes. Fig. 4.8(c) shows the $\mathrm{AC}$ coupling constant of the fibre with $v^{\prime}=3$ and $\theta=7.5^{\circ}$. As the tilt angle increases, the maximum value is teduced and further shifted to the higher radial mode number $m=28$ of $\mathrm{HE}_{3}$ mode ad the firs null in the envelope is, shifted to $m=55$ for of $\mathrm{HE}_{3 \mathrm{~m}}$ mode. 


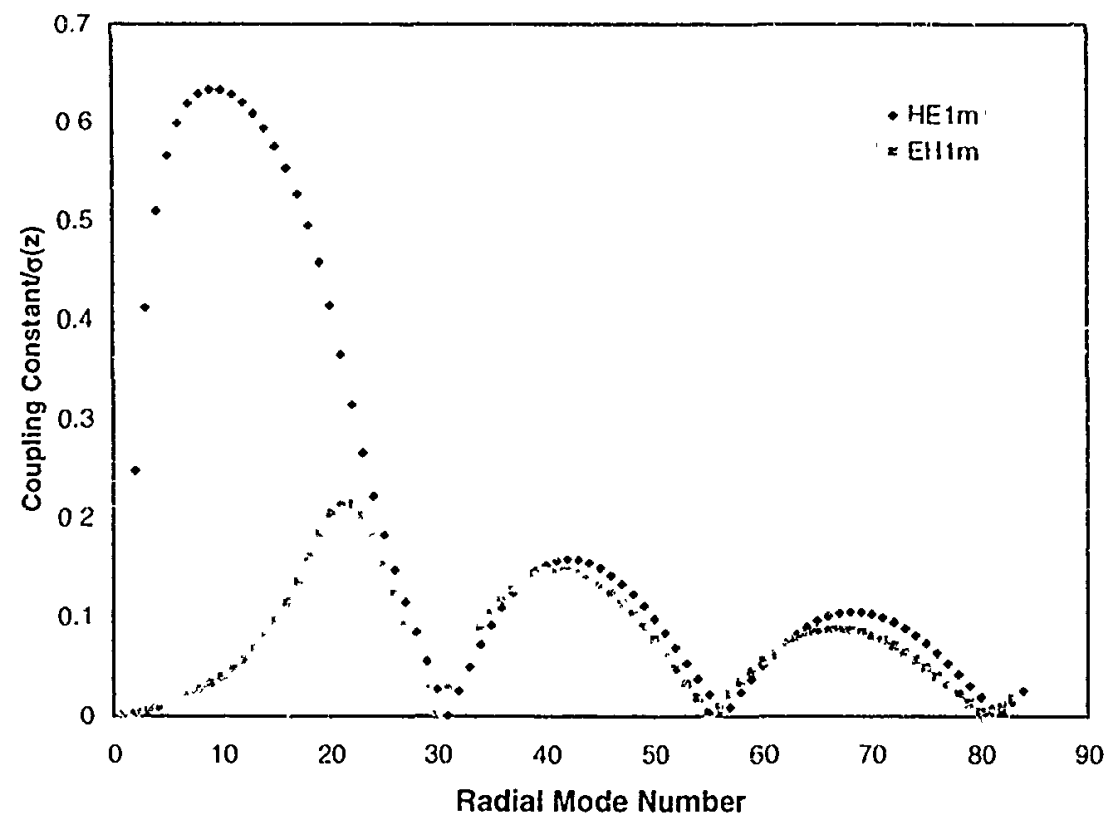

(a)

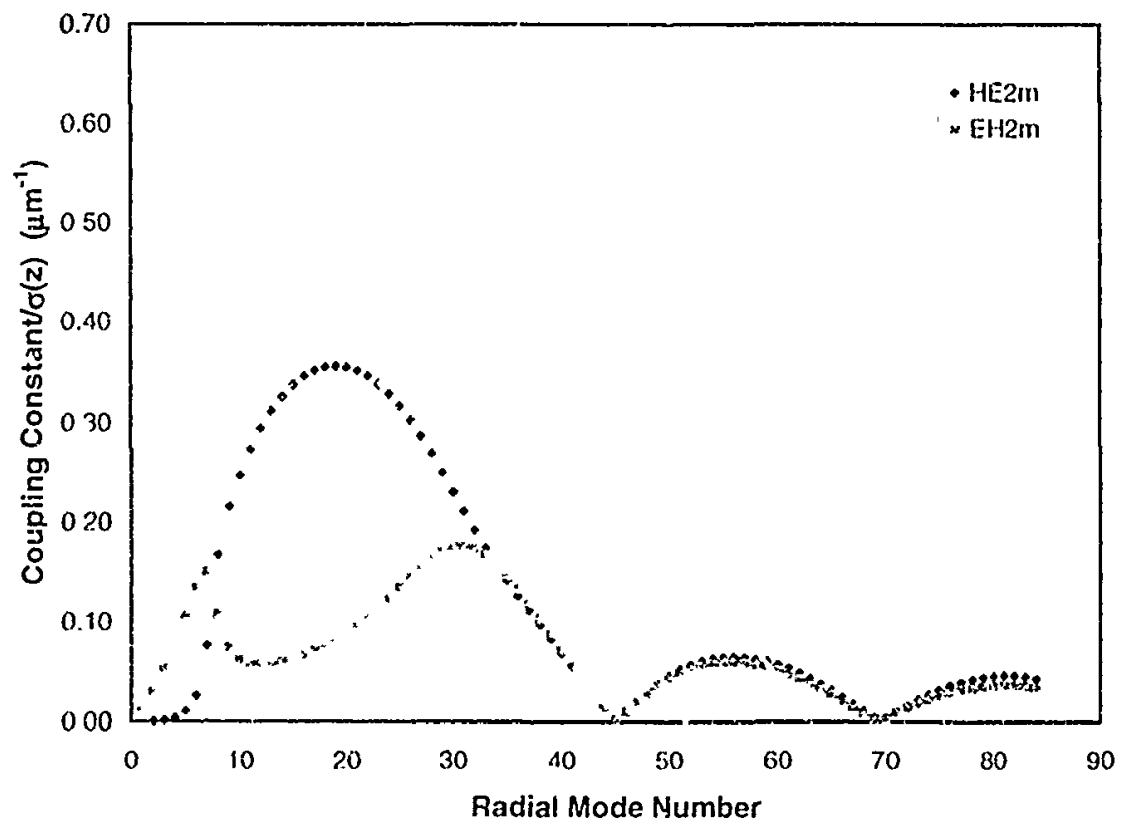

(b) 


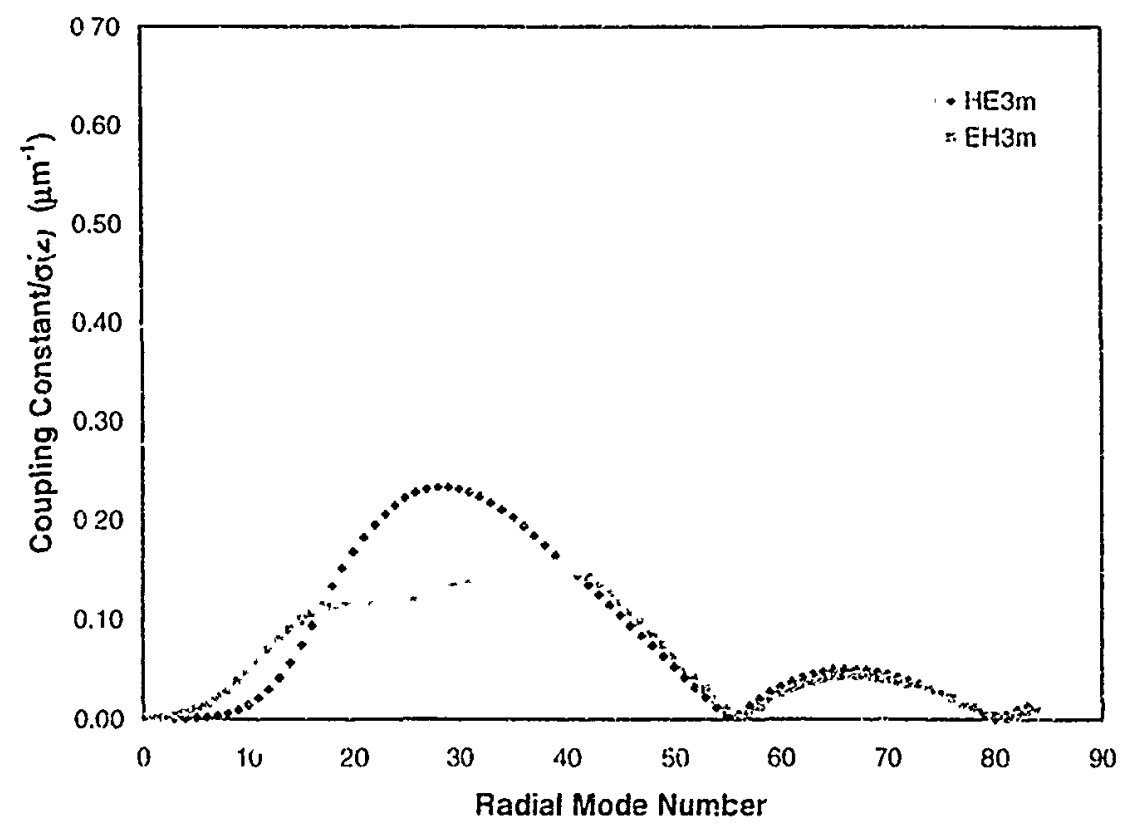

(c)

Figure 4.8: AC coupling constant $2 g_{v^{\prime} m-1} / \sigma(z)$ for cladding modes with (a) $y=1, \theta=0^{\circ}$, (b) $v=2, \theta=5^{\circ}$, (c) $v=3, \theta=7.5^{\circ}$.

\section{b) Case 2}

Fig. 4.9 shows the $\mathrm{AC}$ coupling constant $2 g_{: m-11}$ divided by $\sigma(z)$ for a SMF-28 fibre using Eq. (4.3I) and assuming $M=1$ with different acimuthal numbert and tili angle $\theta$. The fibre has the same parameters as the FBG in SMF-28 fibre discussed before. Fig. 4.9(a) an. (b) shows $I \mathrm{E}_{1 \mathrm{~m}}$ nodes and E $\|_{1 \mathrm{~m}}$ modes with different tit angles. From Fig. 4.9(a) and (b), it can be clealy seen that with the same acimuthal number'. the larger the tilt angle, the smalle: the maximum coupling constunt viue at higher rudbal mode number which corresponds to small iesonance wavelengths. Fig. $4.9(\mathrm{c})$ shows that di low radial mode number for $\mathrm{IE}_{2 m}$ modes w ith tiit digle equals $104^{\prime \prime}$, the coupling constant. are mach larger than for higher mode numier $\mathrm{HE}_{2 m}$ and $\mathrm{SLl}_{2 m \mathrm{i}}$ modes. These modes form 
the su called "ghost mode" that is seen in experimental spectra and as the tilt angle increase the "ghost mode" effect is reduced. Except for the ghos mode region, the coupling constant still follows the rule that the higher azimuthal number $v$ is, the smaller is the maximum coupling constant value.

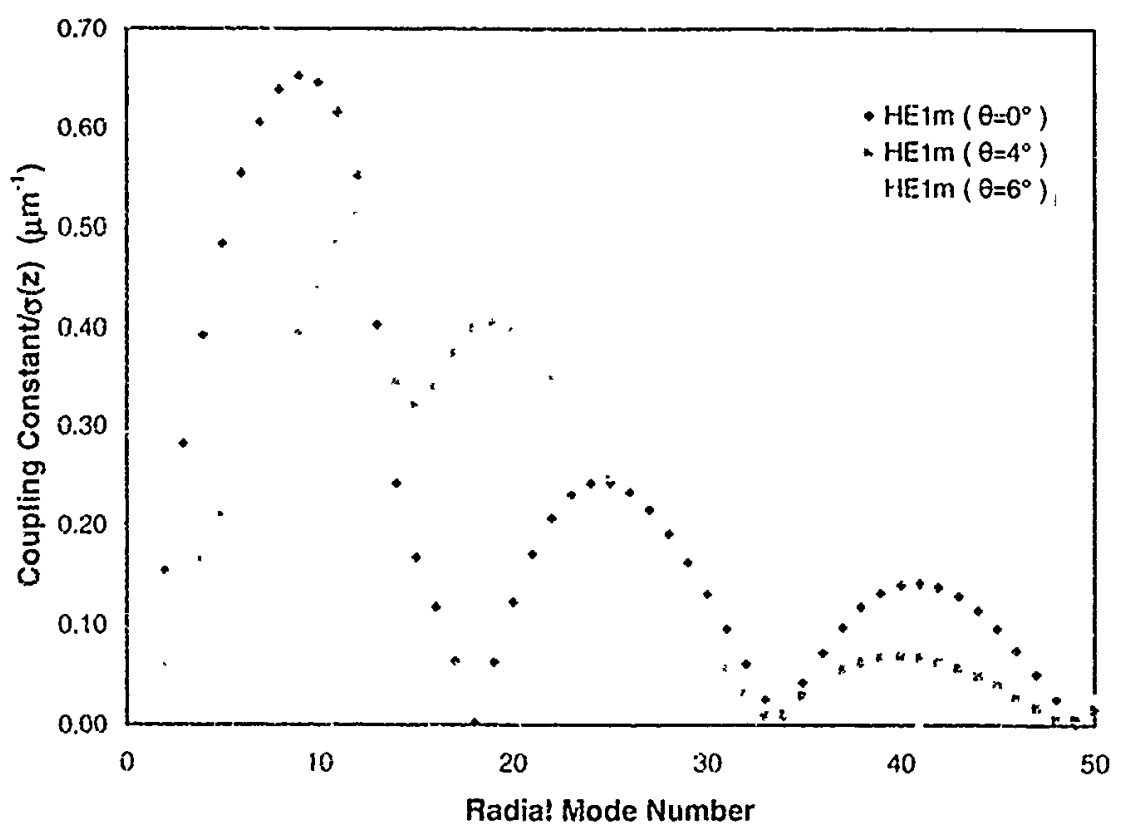

(a)

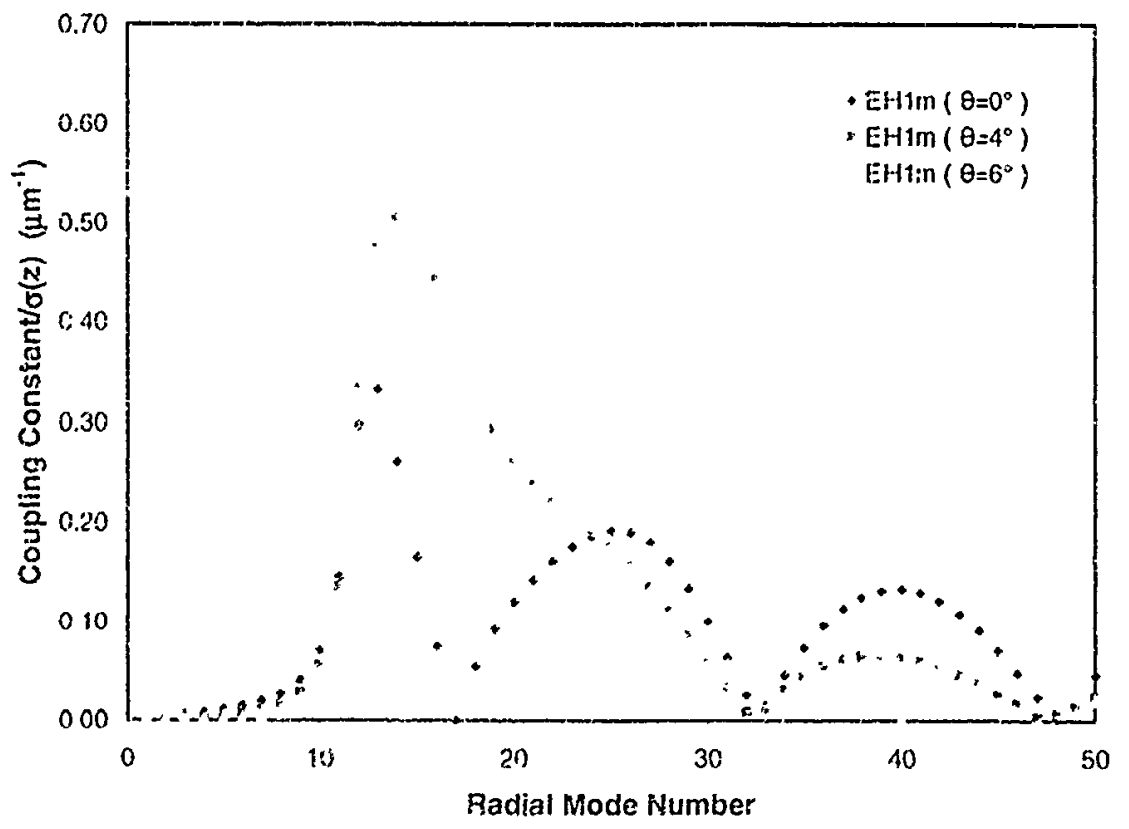

(b) 


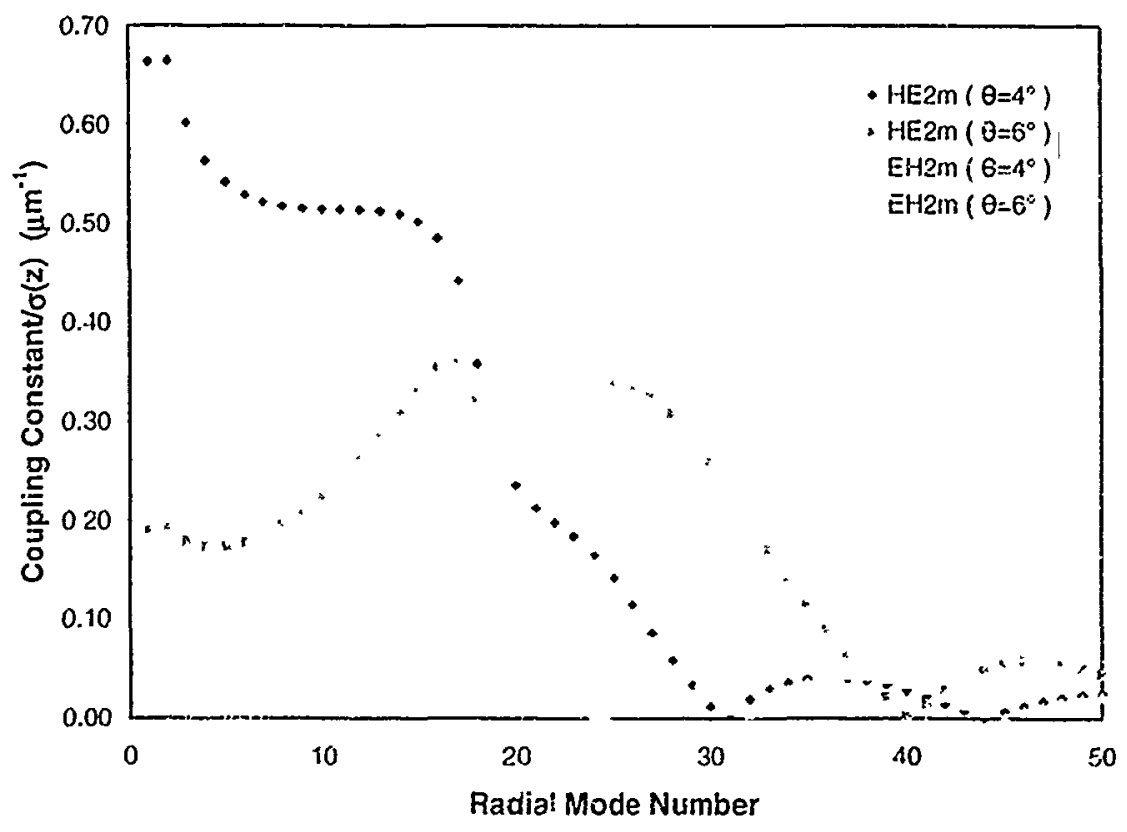

(c)

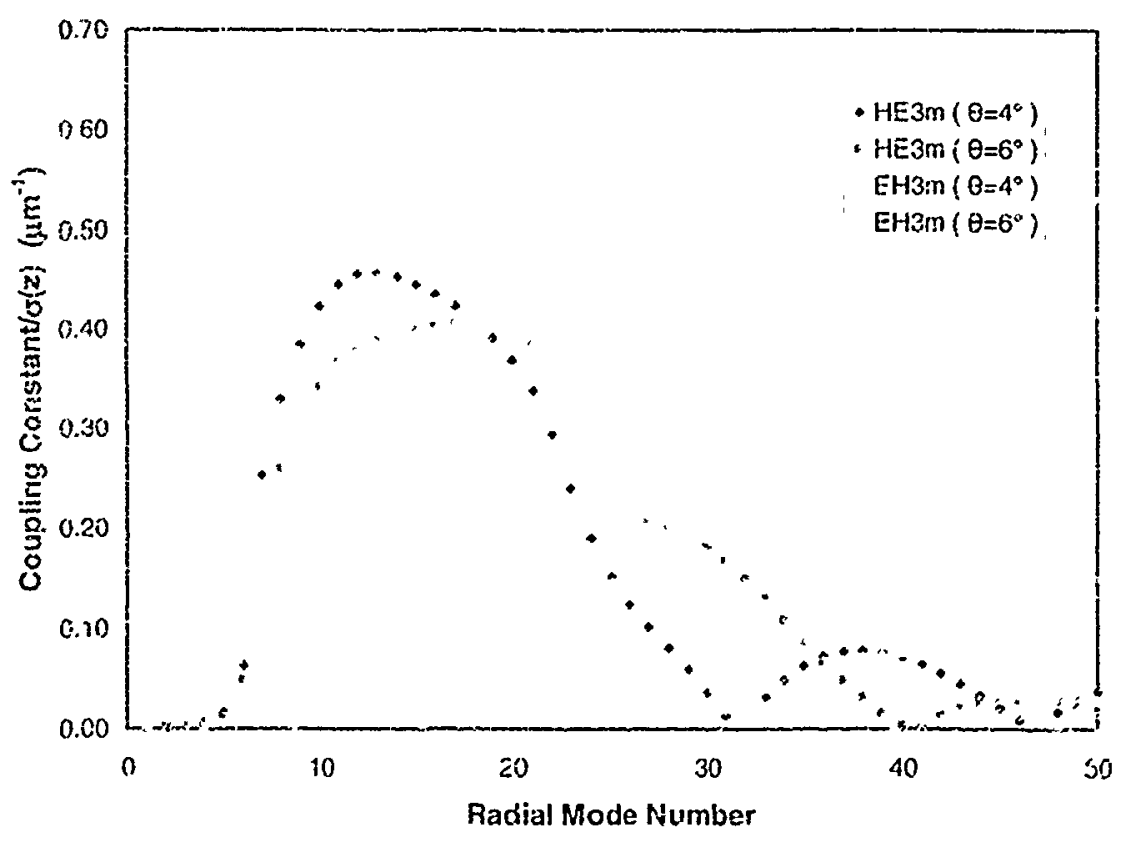

(d) 


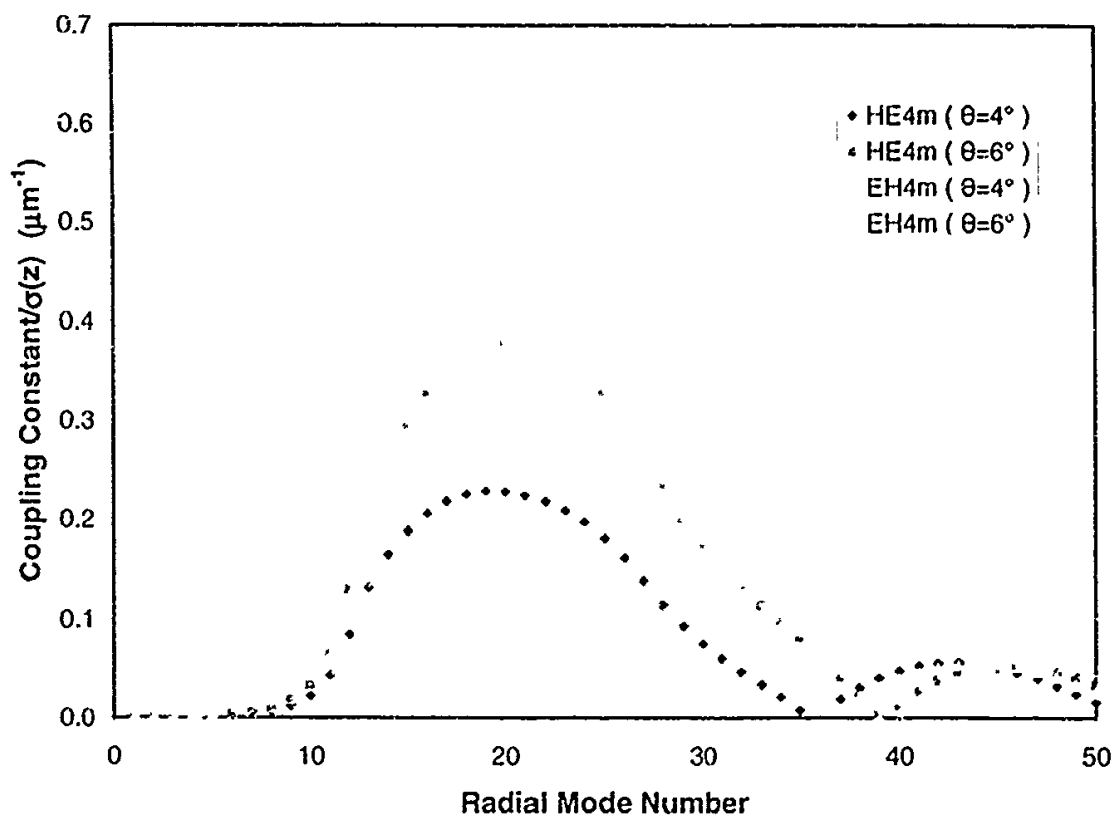

(e)

Figure 4.9: AC coupling constant $2 g_{r^{\prime} m-11} / \sigma(z)$ for cladding modes with (a) $\nu=1$, $\theta=0^{\circ}, 4^{*}$ ano $6^{\prime}$ for $\theta=4^{\circ}$ and $6^{\circ}$, (d) $v \quad 3, \theta=4^{\circ}$ and $6^{\circ}$ (e) $v=4, \theta=4^{\circ}$ and $6^{\circ}$ in SMF-28 fibre.

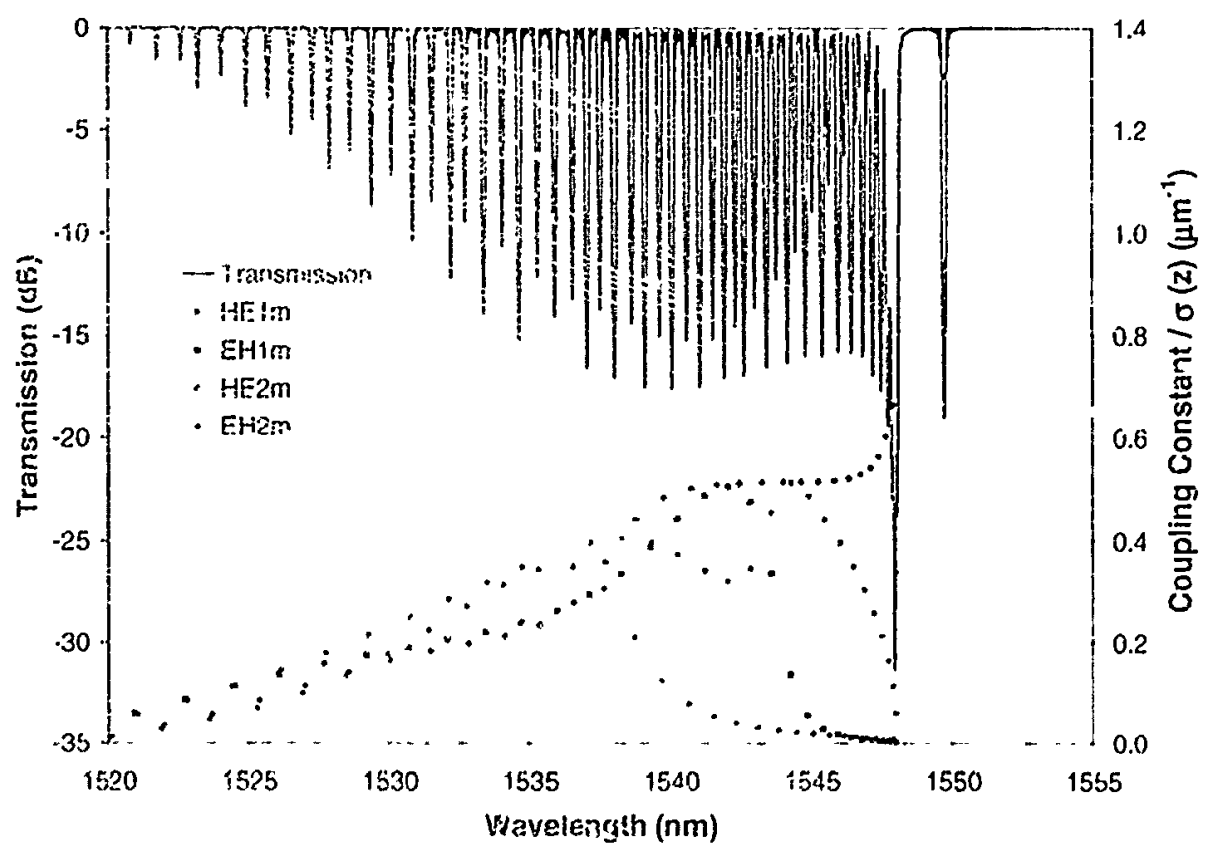

(a) 


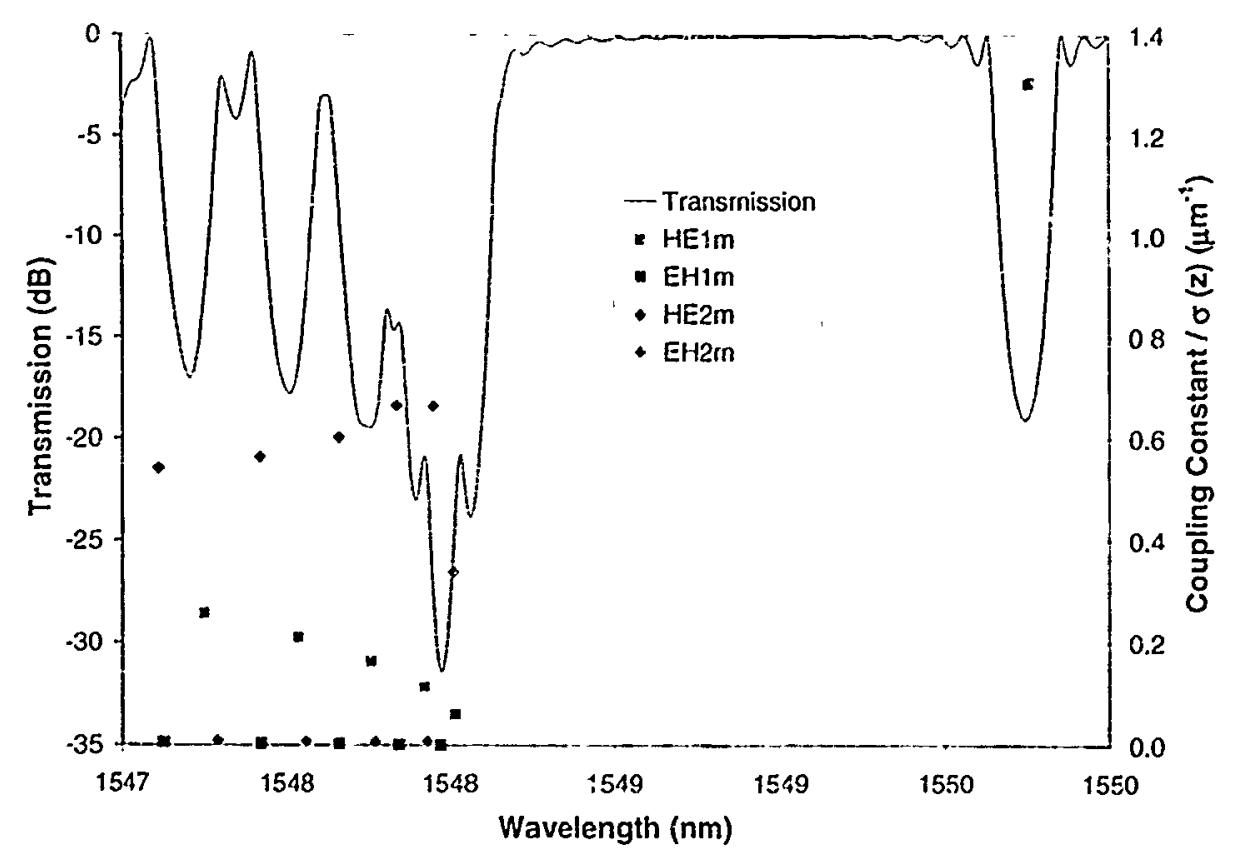

(b)

Figure 4.10: The transmission spectrum of a TFBG in the SMF-28 fibre with a tilted angle $\theta=4^{\circ}$ and simulated $\mathrm{AC}$ coupling constant $2 g_{v m-11} / \sigma(z)$ for core and cladding modes with $v=1$ and 2 in the SMF-28 fibre.

Fig. 4.10 shows the transmission spectrum of one typical TFBG written in SMF-28 fibre with $4^{\circ}$ tilted angle and the simulated coupling constant for a SMF 28 fibre. From Fig. 4.10(a), we can see that simulation result is a good match to the resonance wavelength and the peak value of the cladding modes. The ghost mode region is shown in detail in the of Fig. 4.10(b), the ghost mode resonance has a much broader bandwidth than other cladding mude resonances as the ghost mode resonance is formed by several cladding modes $\mathrm{HE}_{\mathrm{vm}}$ and $\mathrm{EH}_{\mathrm{vm}}$ for buth odd and even azimuthal number $v$ while other cladding mode resonances are formed by cladding modes with either odd azimuthal 
number or even azimuthal number. As shown in Fig. 4.10(b), the ghost mode is mainly formed by $\mathrm{HE}_{21}, \mathrm{HE}_{22}$ and $\mathrm{EH}_{21}$ cladding modes.

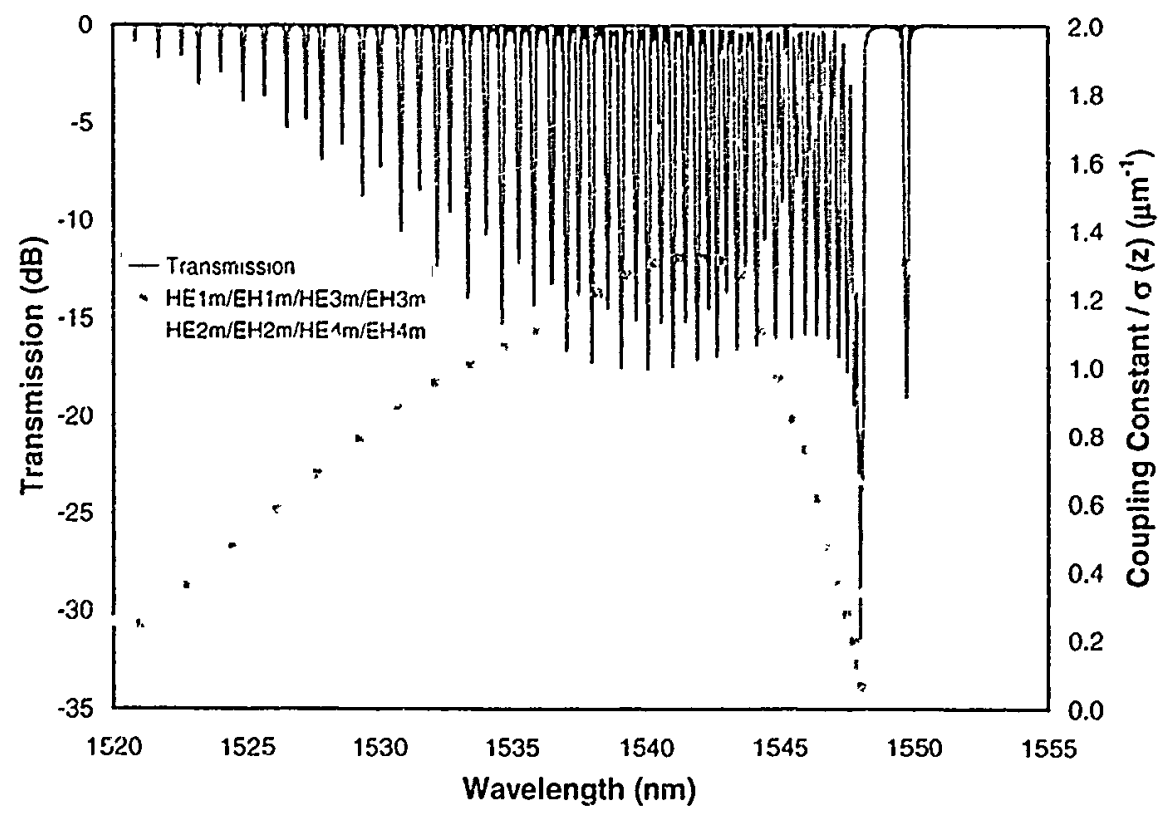

Figure 4.11: The transmission spectrum of a TFBG in the SMF-28 fibre with a tilted angle $\theta=4^{\circ}$ and simulated $\mathrm{AC}$ coupling constant $2 g_{v m-11} i \sigma(z)$ for core and cladding modes with $v=1$ and 2 in the SMF-28 fibre.

Away from the ghost mode or in case where there is no ghost mode, the cladding mode resonances form by set of cladding modes with odd arimuthal number $v$ have larger resonance strength than those are formed by even azimuthal number $v$. This shows in the ransmission spectrum: the nearby uladding resonarices are one trong, one weak alternativcly. The strong one is formed by $\mathrm{HE}_{1 \mathrm{~m}} / \mathrm{EH}_{1 \ldots}, \mathrm{HE}_{3 \mathrm{~m}} / \mathrm{HE}_{2_{2 m}} \cdots$, and the weak one is formed by $\mathrm{HE}_{2 m} / \mathrm{EH}_{2: m}, \mathrm{HE}_{4 \mathrm{~m}} / \mathrm{HL}_{4}, \cdots, \cdots$. If there is a shost mocic in the transinission spectrum, then in the low oricr cladding mode region, the cludding mode ressnances with even azimuthal number $v$ have larger resonance verngth than those d'e formed by 
odd azimuthal number $v$ as shown in Fig. 4.11. Each coupling constant of the cladding modes shown in Fig. 4.11 is a superposition of four cladding modes: (i) for odd azimuthal number $v$, it is superposition of $\mathrm{HE}_{1 \mathrm{~m}} / \mathrm{EH}_{1 \mathrm{~m}} / \mathrm{HE}_{3 \mathrm{~m}} / \mathrm{EH}_{3 \mathrm{~m}}$ modes at the resonance wavelength of $\mathrm{HE}_{1 \mathrm{~m}}$, and (ii) for even azimuthal number $v$, it is a superposition of $H E_{2 m} / \mathrm{EH}_{2 \mathrm{~m}} / \mathrm{HE}_{2 \mathrm{~m}} / \mathrm{EH}_{2 \mathrm{~m}}$ modes at the resonance wavelength of $\mathrm{HE}_{2 \mathrm{~m}}$. The coupling constant of the core mode shown in Fig. 4.10 is much larger than for the other cladding modes, but the cladding mode resonances are formed by a superposition of several cladding modes, therefore the total cladding mode resonance peak can be inuch larger than the core resonance such as ghost mode or comparable with the core mode resonance as other cladding mode resonances seen in the TFBG spectrum of Fig. 4.1\}.

\section{c) Case 3}

Fig. 4.12 shows the coupling constant with different azimuthal number $v$ and tilted angle $\theta$ for a SMF 28 fibre with a $20 \mathrm{~nm}$ gold coating and immersed in water. The fibre parameters are: $n_{1}=1.4504, n_{2}=1.444024, n_{3}=0.55-j 11.3, n_{4}=1.33, r_{1}=4.15 \mu \mathrm{m}$, $r_{2}=62.5 \mu \mathrm{m}, r_{3}=62.52 \mu \mathrm{m}(d=20 \mathrm{~nm})$, and $\lambda=1.55 \mu \mathrm{m}$. The cladding modes in a fibre with a thin metal codting can show some plasmon mode characterisits. When the cladding modes show plasmon mode characteristics, they hate strong $f_{1}$, wh surrounding the metal surfaces which means that the plasmon inode will show ldreg biss compared with other cladding mudes. Fiom Fig. 4.12 we can sec the maximum low nappen ne. $1497 \mathrm{~nm}$ which is $53 \mathrm{~nm}$ away from the Bragg wavelength $1550 \mathrm{~nm}$. As we cai $2 \mathrm{x}$. Th coupling constants at $1497 \mathrm{~nm}$ are very small with the tilt angle of 4 and 6 . Se a lat:- 
tilted angle such as $10^{n}$ is needed for TFBG, to have a larger coupling constant in the plasmon mode region. From that, we can see that for index sensing purposes we can design the TFBG with specific angles for different index sensing regions. The larger the surrounding refractive index is, the smaller:, the tilt angle. Similarly as in the case for FBG, comparing with the SMF-28 without a gold coating (Fig. 4.9), the maximum coupling constant is reduced by more than $20 \%$.

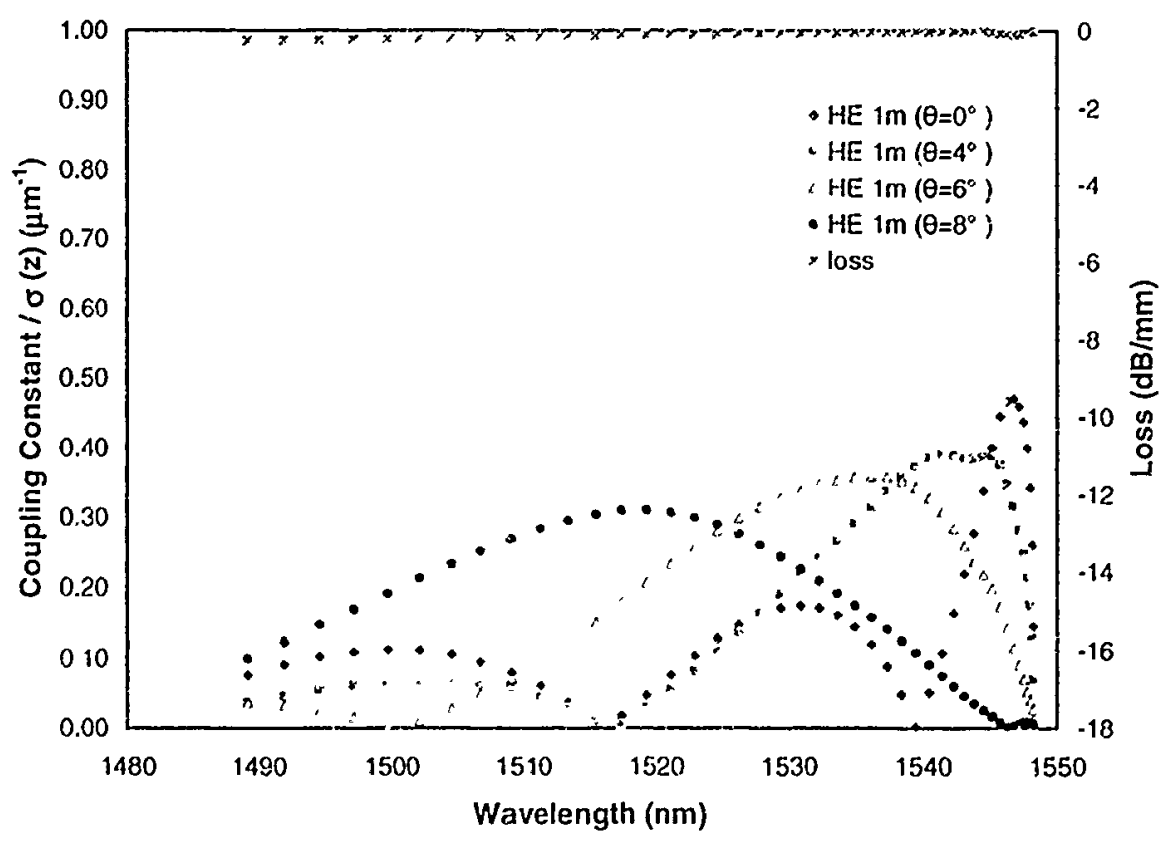

(a) 


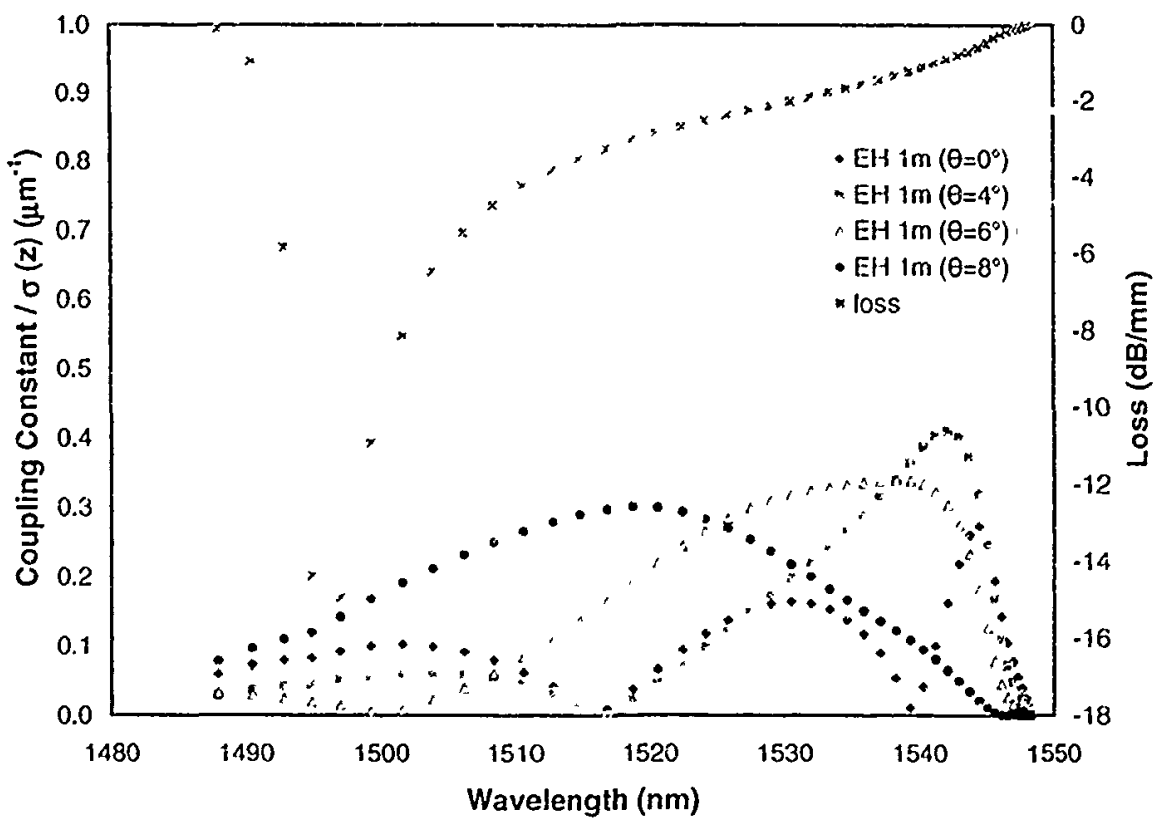

(b)

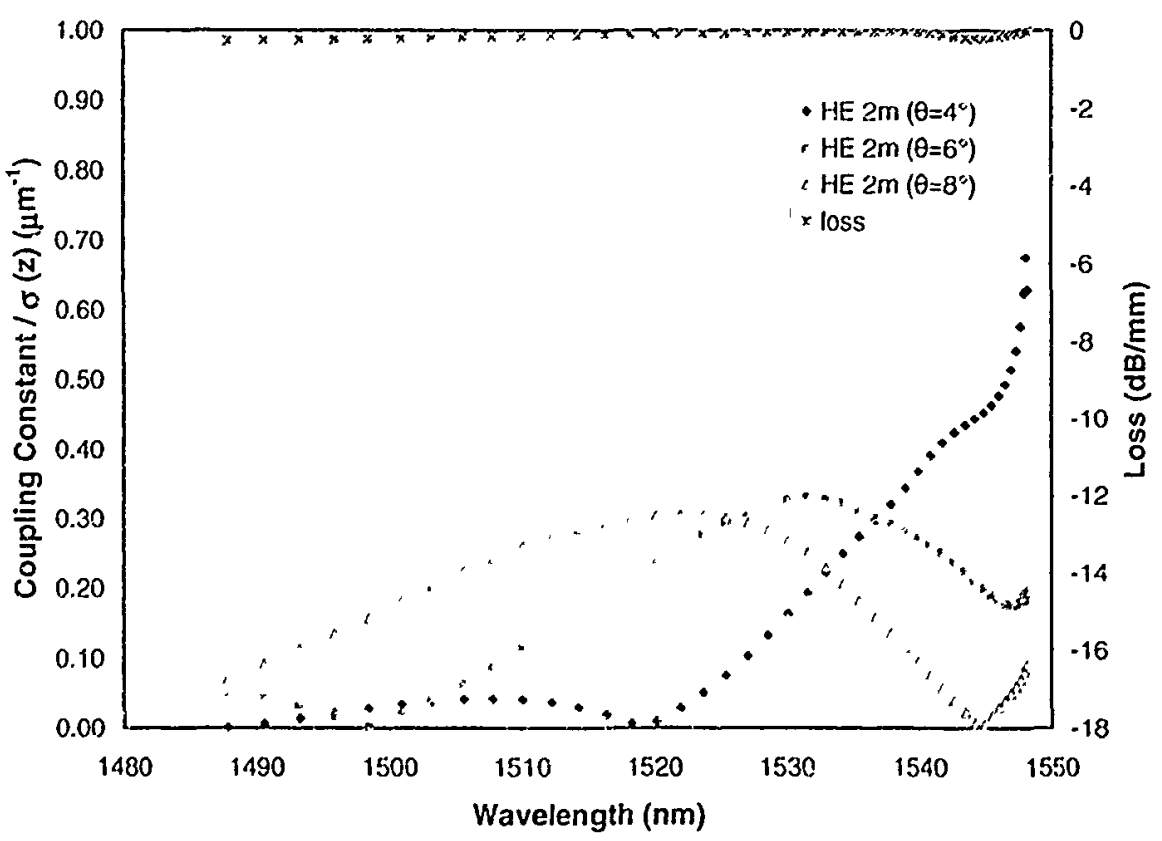

(c) 


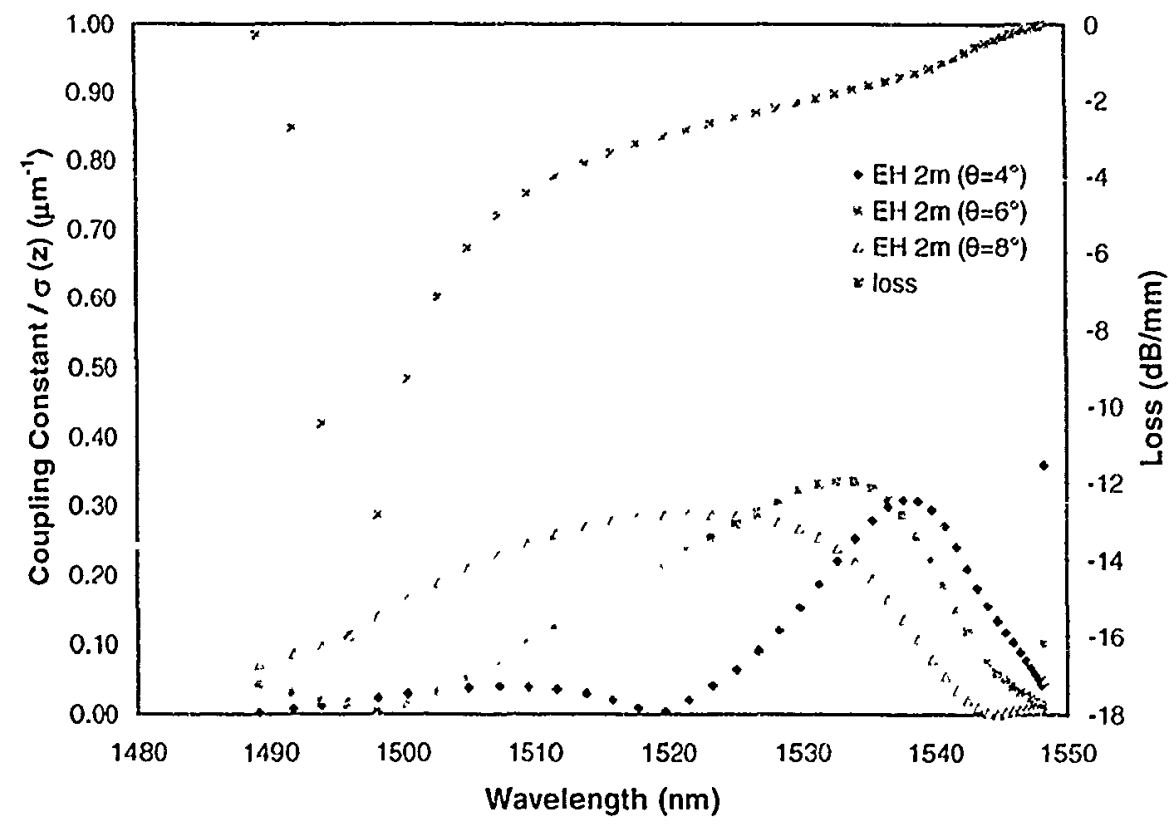

(d)

Figure 4.12: AC coupling constant $2 g_{v m-11} / \sigma(z)$ for cladding modes (a) $\mathrm{HE}_{1 \mathrm{~m}}$ modes: $\theta=0^{\circ}, 4^{\circ}$ and $6^{\circ}$, (b) $\mathbf{E H}_{\mathrm{Im}}$ modes: $\theta=0^{\circ}, 4^{\circ}$ and $6^{\circ}$, (c) HE $2 \mathrm{~m}$ modes: $\theta=4^{\circ}$ and $6^{\circ}$, (d) $\mathrm{EH}_{2 \mathrm{~m}}$ modes: $\theta=4^{\circ}$ and $6^{\circ}$, in SMF-28 fibre with $20 \mathrm{~nm}$ gold coating and $\mathbf{n}_{4}=1.33$.

\section{d) Case 4}

Fig. 4.13 (a) shows the experiment result of a $6^{\circ}$ TFBG with a $15 \mathrm{~nm}$ gold coating immersed in a sucrose solution with $\mathrm{n}_{1}=1.4378$ [45]. It is clear that strong resonances in the $\lambda_{\mathrm{P}}$ region are low loss cladding mode resonances formed surrounded by the leaky mode resonances on the low wavelength side and plasmon mode resonances on the high wavelength side. Both leaky modes and plasmon modes show large loss. The plasmon resonances are very weak and their resonance wavelength is hard to track accurately. Fig. 4.13 (b) shows the simulation results for a $6^{n}$ TFBG with $20 \mathrm{~nm}$ gold coating with $n_{s}=1.415$. As we can sec from Fig. 4.13 (b), the coupling constant of plasmon modes do not show much difference with those of other cladding modes even with the low loss 
cladding mode resonances in $\lambda_{P}$ region. Therefore the small resonance amplitude of the plasmon modes shown in the transmission spectrum is mainly due to the heavy loss of the plasmon modes. It is very clear that the low loss cladding mode resonances in $\lambda_{\mathrm{P}}$ are formed by those cladding modes before the cutoff. As the mode cutoff is directly related with the effective index of the mode and SRI, as the SRI increases, the cladding mode resonances in the higher walelength will show decreasing loss characteristics and the wavelengths of the high amplitude resonances linearly increase with SRI. Fig. 4.13 (c) shows $\mathrm{HE}_{\mathrm{Im}} / \mathrm{EH}_{\mathrm{Im}}$ cladding mode loss el.velope with the different SRIs. As we can see the wavelength of the plasmon mode loss shift towards the red as the SRI increases. The plasmon mode resonance wavelength shift is close to linear.

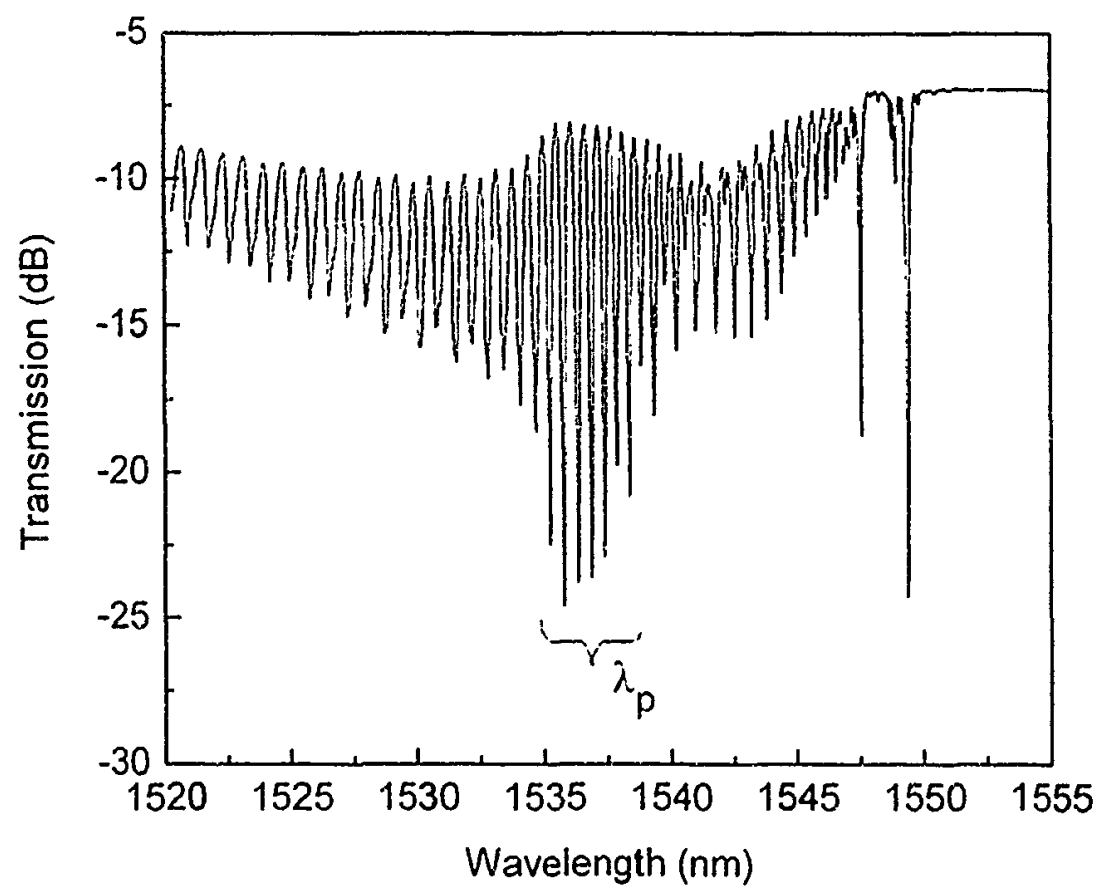

(a) 


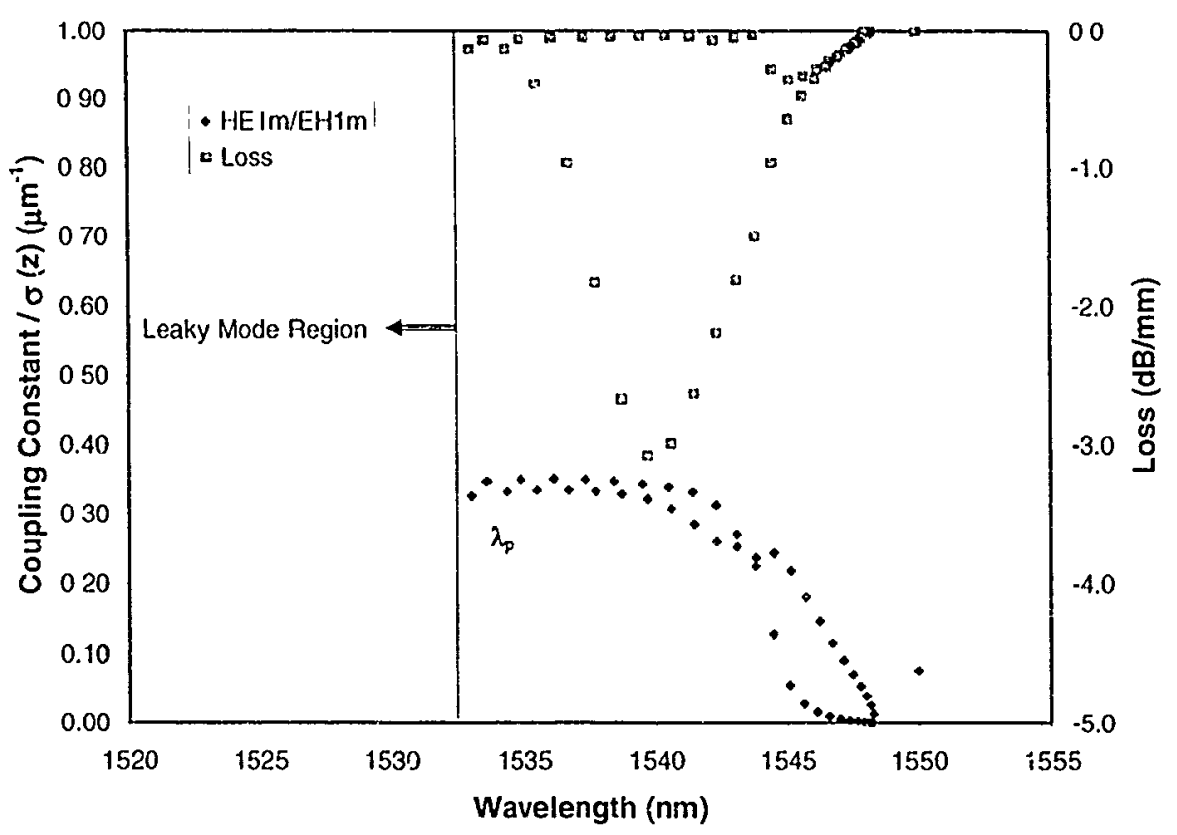

(b)

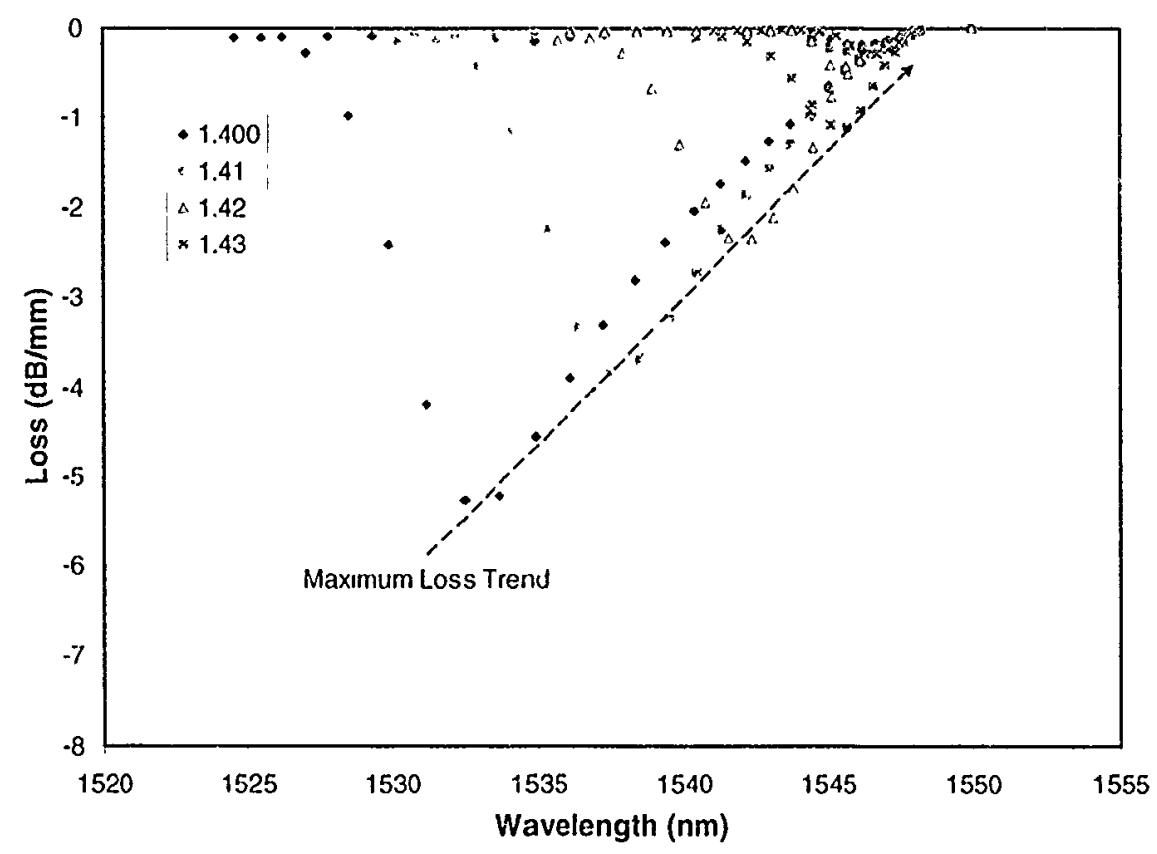

(c)

Figure 4.13: (a) The transmission spectrum of TFBG with a $15 \mathrm{~nm}$ gold coating immersed in a sucrose solution with $n_{p}=1.4378$ [45], (b) AC coepling constant $2 g_{v m-11} / \sigma(z)$ and mode propagation loss for cladding $\mathrm{HE}_{\mathrm{lm}}$ with $0=6^{\circ}$ and $\mathrm{n}_{\mathrm{S}}=1.415$, (c) Mode loss envelope of HE1m modes with different SRI. 
Fig. 4.14 (a) shows the simulated plasmon mode resonance peak shifts with the SRI. Comparing with the experiment result of low loss cladding mode resonances of $\lambda_{p}$ shown in Fig. 4.14 (b) [45], the slope is about $10 \%$ difference. As we can see that $\lambda_{p}$ is about $10 \%$ higher index sensitivity than plasmon mode resonance, and their resonances are relatively casier to track than the plasmon mode resonance. Therefore the low loss cladding mode resonances shown in the transmission spectrum: of TFBG with a thin gold coating not only take the advantage of plasmon mode for its high index sensitivity, but also shows the easy way to tracking. It is a realiy good candidate for high index sensitivity biomedíal sensor applications.

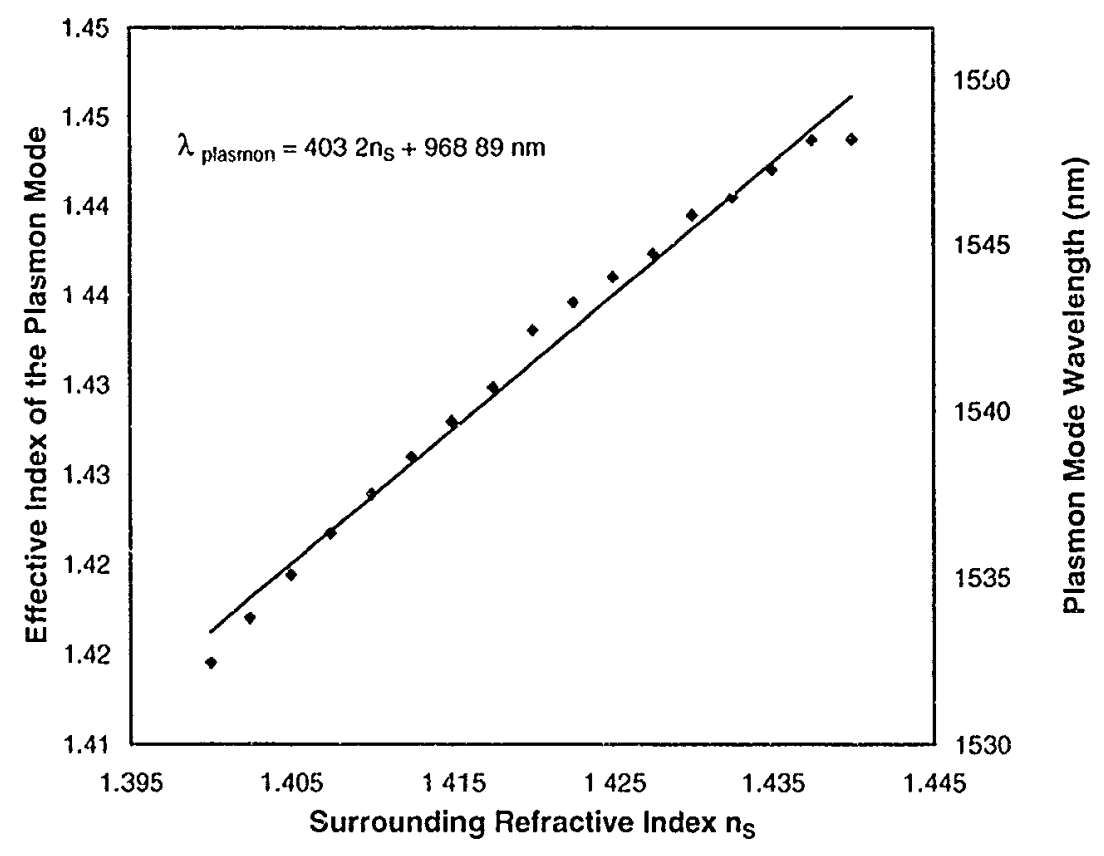

(a) 


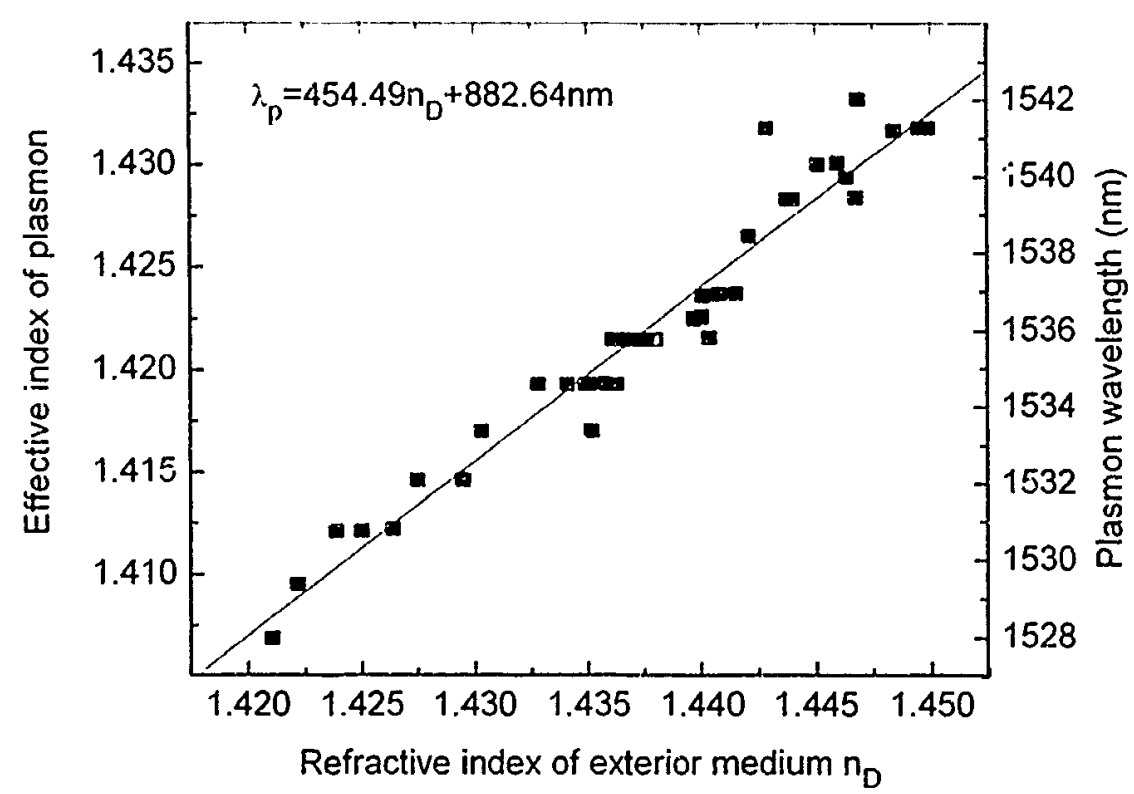

(b)

Figure 4.14: (a) Simulated dependence of the plasmon peak wavelength $\left(\lambda_{\text {plasmon }}\right)$ and corresponding cladding mode effective index on the SRI, (b) measured dependence of the resonance peak wavelength $\left(\lambda_{\mathrm{p}}\right)$ and corresponding cladding mode effective index on the refractive index of th: external medium at $589 \mathrm{~nm}\left(\mathrm{n}_{\mathrm{f}}\right)$ [45].

\subsection{Discussions}

The transmission spectrum envelope and each core and cladding mode resonance wavelength of FBG and TFBG can be predicted hy calculating the core and cladding mode coupling constants, but exact resonance strength and transmission spectrum need to solved by coupled mode equations. For a weak FBG, due to the narrow bandwidth $(\sim 0.1$ $\mathrm{nm}$ ) of cladding mode resonances and large cladding mode resonance wavelength separations from $0.3 \mathrm{~nm}$ to $1.6 \mathrm{~nm}$, then we can keep only the closest resonances in simulations and unly two mode coup!ings: the core mije and one cladding mode closest to the resonance wavelength. A closed form solution is available [20] of Eq. (4.8) for the transmission spectrum of uniform gratings. If the grating is not uniform or not weakly 
modulated, then Eq. (4.8) needs to be solved by numerical methods. One widely used is Runge-Kutta method [40].

For the TFBG, because each cladding mode resonance is formed by several cladding modes they have the azimuthal number $v$ either odd $(v=1,3,5 \cdots)$ or even $(v=2,4,6 \cdots)$, so the two mode coupling equation and its solution can not be used for TFBG spectrum simulations and the Runge-Kutta method is needed.

Leaky mode resonances in TFBG with a thin layer gold coating show a big difference with those in TFBG without a thin layer gold coating. Leaky mode resonances in fibres without metal coating normally show heavy loss and very large resonance bandwidth and the high order leaky modes decay fast. However when the fibre has a metal coating, leaky mode resonances have a similar bandwidth as other guided cladding mode resonances. Further exploring those leaky mode characteristics may bring a new set of sensors, but more analytical work needs to be done to better understand the mechanism inside.

As we see that the cladding mode resonances can be distributed over more than 30 nm wavelength span in the transmission spectrum of TFBG, the wavelength dependent material dispersions of $\mathrm{SiO}_{2}$ and $\mathrm{Ge}-\mathrm{SiO}_{2}$ cannot be ignored in the coupling constant calculations and transmission spectrum simulations for high order cladding modcs. We will discuss it in detail in the next Chapter.

\subsection{Summary}

In this Chapter, the mode coupling between core and cladding modes with or without a small tilt angle are analyzed. New formulations for calculating the coupling constant are 
derived. The numerical results are compared with the literature and good agreement is obtained. The coupling constants of SMF 28 fibre are well matched with the experimental results. By simply calculating the coupling constants we can predict the FBG spectrum characteristics. The effect of the fibre core radius in FBG and TFBG is discussed. For TFBG, the tilt angle effect for the transmission envelope is discussed as well. The SMF-28 fibre with a thin gold coating is analyzed and calculated in detail. Some cladding modes in SMF-28 fibre with a thin gold coating show plasmon mode characteristics and they are very interesting for biosensor applications. This work provides a very useful tool to analyze and understand what and why the specific spectrum character happens in such kind of structure. Therefore it can help designing new types of TFBG and FBG for high sensitivity index sensor and use them for biomedical sensor purposes. 


\section{Chapter 5}

\section{Sensing Characteristics of Core and Cladding Modes in}

\section{Single Mode Fibres}

\subsection{Introduction}

Fibre Bragg grating (FBG) sensors have a wide range of applications such as pressurestrain sensors, temperature sensors, micro-bending sensors and external refractive index sensors [8-13]. As these optical sensors are inherently immune from electromagnetic interference and chemically inert, they are very attractive in bio-chemical applications and hazardous surroundings.

The sensing mechanism most often used in FBGs arises from the fact that the reflection wavelength for the forward propagating core mode varies linearly with temperature and strain. Since the wavelength can be measured. with an accuracy of 10 pm relatively easily near $1550 \mathrm{~nm}$, this represents a relative resolution of about $6 \mathrm{ppm}$. A variant of the same concept uses so-called Long Period Gratings (LPG) where coupling occurs between the forward propagating core mode and forward propagating cladding modes. In this case, the sensitivity of the resonance wavelength to perturbations can be greatly enhanced for some of the cladding modes. Furthermore, since LPGs involve cladding modes there has been great interest in using thesc for refractive index sensing by 
immersing the fibres in the medium to be measured. Special absorbing coatings can also be used to detect chemicals or liquids through the refractive index changes (or volume changes) induced in the coatings. However, the spectral response of LPG resonances is rather brodd (width greater than $10 \mathrm{~nm}$ ) making high accuracy measurements of small wavelength changes more difficult than with FBGs. Ideally, such refractive index sensors should be able to distinguish different kinds of perturbations, and insersitivity to temperature is often particularly desirable. A problem with both FBG and LPG sensors is that they are intrinsically quite sensitive to temperature, with resonance wavelengths drifting by about $10 \mathrm{pm} /{ }^{\circ} \mathrm{C}$, unless special bulky packaging is used to athermalize the device. In order to circumvent this problem in refractive index sensors, devices proposed so far have involved combination of gratings in one sensor such as two different types of fibre Bragg gratings [9], two fibre Bragg gratings with different cladding diameters [49] and a long period grating (LPG) with a Bragg grating [50]. In such cases, the differential sensitivity of the two gratings to temperature and the desired measurand is used to discriminate between the two perturbations.

In this Chapter, we propose and demonstrate a single weakly tilted fibre Bragg grating (TFBG) sensor which performs the same function as a double FBG sensor or as a LPG-FBG combination. In the TFBG, both a core mode resonance and several cladding mode resonances appear simultaneously as shown in Fig. 5.1. This has several advantages. The cladding mode resonances are sensitive to the external environment (refractive index, deposited layer thicknesses, etc.) and to physical changes in the whole fibre cross-section (shear strains arising from bending for instance), while the core mode 
(Bragg) resonance is only sensitive to axial strain and temperature. We will show that the temperature dependence of cladding modes is similar to that of the core modes, sc that the effect of temperature can be removed from the cladding mode resonance by monitoring the wavelength difference between the cors mode resonance and selected cladding mode resonances. Using this technique, a temperature-independent strain and surrounding refractive index (SRI) sensor can be made.

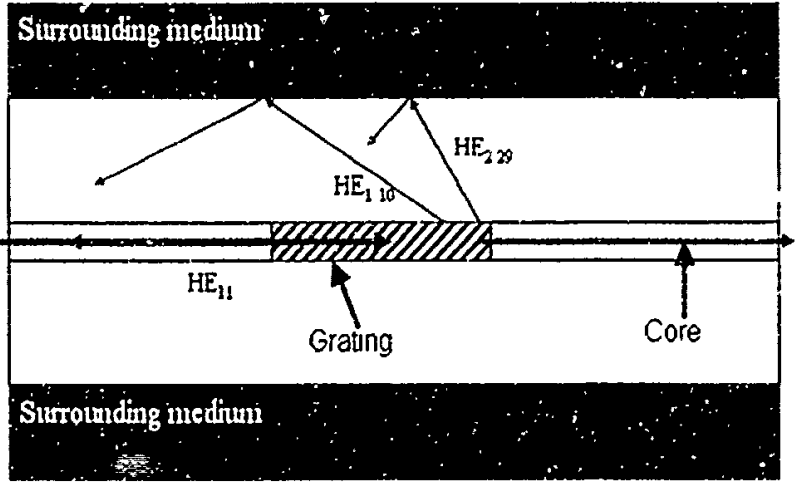

(a)

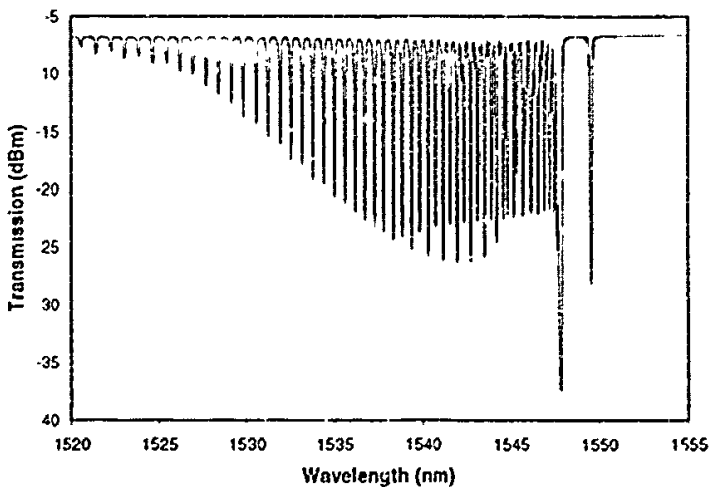

(b)

Figure 5.1: (a) TFBG schematic; (b) the transmission spectrum of a TFBG.

Another feature which may constitute an advantage of the TFBG over the LPG to couple to cladding modes is that the resonances are as narrow as those of a FBG, i.e. of the order of a few hundred pm, instead of several tens of nm for LPGs. Therefore, wavelength interrogation for TFBG occurs over narrow wavelength ranges and makes it possible to use commonly available light sources, detectors, couplers and multiplexers. In particular, a typical entire TFBG spectrum fits easily within the standard telecommunication bands $(1530-1560 \mathrm{~nm})$. The concept of using claciding mode resonances from a TFBG was originally proposed by Laffont and Ferdinand [23] but their 
scheme was limited to monitor the envelope of the resonances as the SRI changes and did not use the core mode resonance for self-referencing with regards to temperature. Our approach is the first in the world to make use of the resonance positions and/or strengths individually, which allows us to extract multiple sensing parameters from a single sensor. To be precise, a multi-functional sensor can be developed by monitoring several cladding mode resonances (or groups of resonances) which have different sensitivities to the external environment changes. For instance, bending affects mainly low order cladding modes [10], while SRI increases affect higher order cladding modes preferentially [23], and neither perturbation affects the core mode resonance.

\subsection{Experimental Conditions}

\subsubsection{Fabrications of FBGs and TFBGs}

The experimental FBGs and TFBGs are written in nydrogen-loaded CORNING SMF-28 fibres using $193 \mathrm{~nm}$ ArF excimer laser light or $248 \mathrm{~nm} \mathrm{KrF}$ excimer laser light with a phase mask to generate the grating pattern. The setup for writing TFB Fs is shown in Fig. 5.2. In this configuration, the internal tilt angle 0 of the grating planes can be calculated from the mask tilt angle $\alpha$ by applying Snell's law of refraction at the air-glass interface of the fibre. The resonance wavelengths of the Bragg and cladding modes are determined by the projection of the grating period of the mask. used on the filme axis and on the effective indices of each mode. 


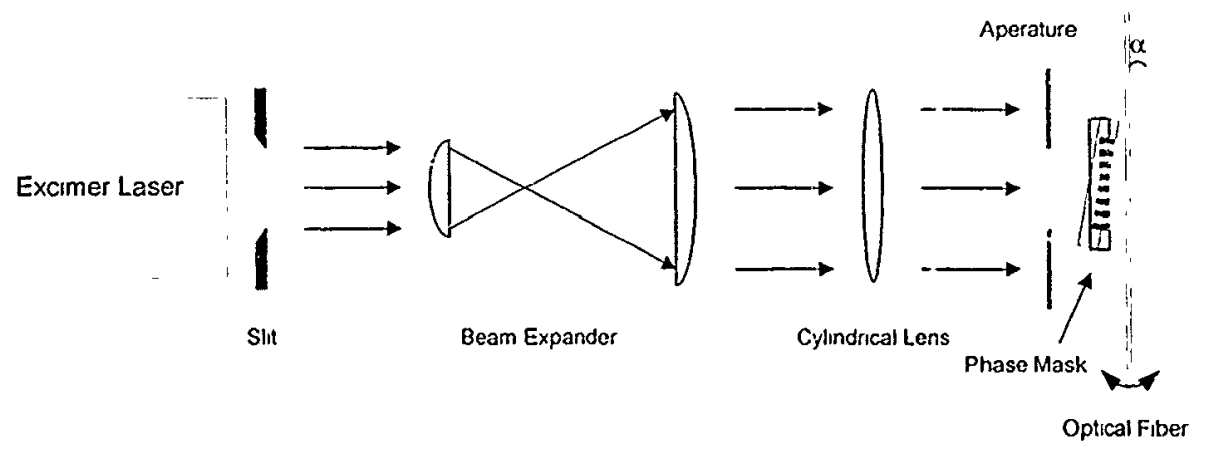

Figure 5.2: The top view of setup for TFBG written

\subsubsection{The Transmission Spectrum Characteristics of FBG and TFBG in Single Mode Fibres}

Fig. 5.3 shows transmission and reflection spectra of a FBG and a TFBG written in a SMF-28 fibre without/with a tilt angle $\theta=4^{\circ}$ using $193 \mathrm{~nm}$ ArF excimer laser light. From Fig. 5.3 (a) we can see that the cladding mode resonances are very weak comparing with the core mode (Bragg) resonance, as we analyzed in Chapter 4. In most of telecommunication applications, suppressing the cladding mode resonances are desired for a clear FBG transmission spectrum; in sensor application, the core mode resonance in transmission and reflection spectra are widely used for temperature and strain sensor, and an array of FBGs is also widely used for structure health monitoring, it is possible to track the cladding resonances in a FBG for sensor applications, but it is unpopular because the cladding mode resonances are too weak. 


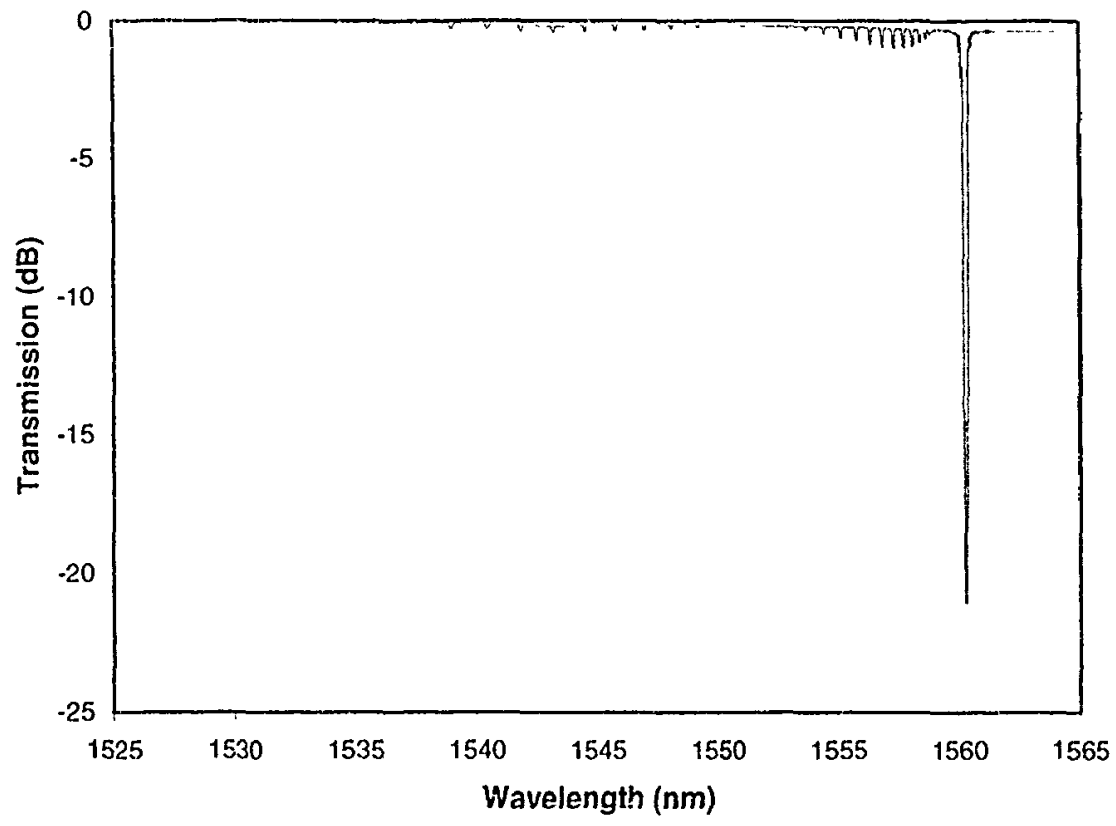

(a)

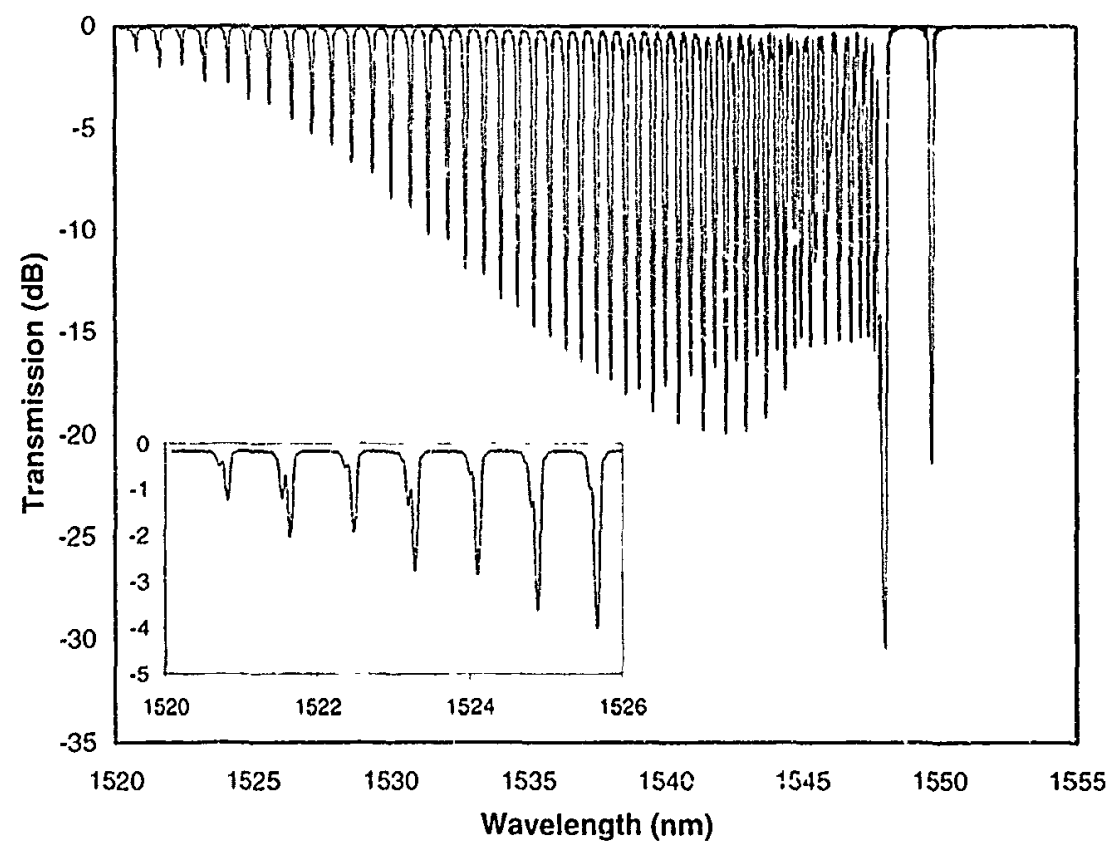

(b) 


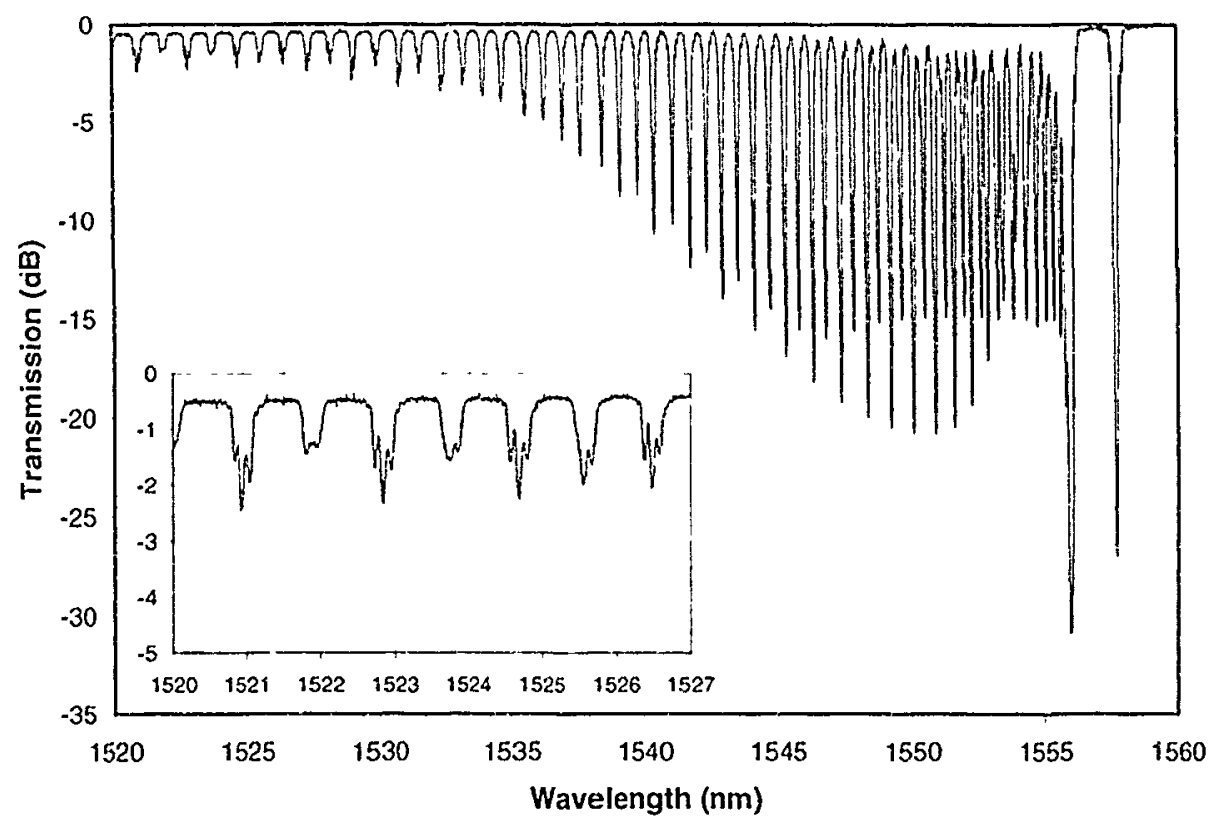

(c)

Figure 5.3: The transmission spectra of EBG and $4^{\circ}$ tilted FBGs written in SMF-28 single mode fibres: (a) FBG, (b) TFBG written by $248 \mathrm{~nm}$ KrF excimer laser light; (b) TFBG written by $193 \mathrm{~nm}$ ArF excimer laser light.

Fig. 5.3 (b) shows the transmission spectrum of the TFBG written in a SMF-28 fibre with tilt angle $\theta=4^{\circ}$ by $248 \mathrm{~nm} \mathrm{KrF}$ excimer laser light. The $4^{\circ}$ tilt angle was chosen to make the Bragg resonance and the maximum cliding mode resonance have similar resonance amplitude. When the tilt angle increases, the Bragg resonance amplitude reduces and the maximum cladding mode resonance increases and shift to low wavelength region. Now the cladding resonance strength is compardble with the core mode resonance strength. An important feature of the small tilt angle is the presence of a strong "ghost niode" resonance, immediately to the left of the Bragg resonance. This ghost mode is made up of several low order cladding modes as we discussed in previous Chapter. The ghost mode is known to be very sensitive to bending [51], but very little to outside refractive 
index [52]. The spectrum of the shorter wavelength region, which is about $23 \mathrm{~nm}$ away from the core resonance shows that in this region the cladding resonances become double peaked. This is due to the fact that for higher order cladding modes, the fields are less confined in cladding layer and therefore the degeneracies of the LP mode approximations are split up into individual full vectorial modes of the fibre. The irset of Fig. 5.3(b) shows the transmission spectrum of the short wavelength region in detail. Fig5.3(c) shows the transmission spectrum of the TFBG written in a SMF-28 fibre with tilt angle $\theta=4^{\circ}$ by $193 \mathrm{~nm}$ ArF excimer laser light, which shows more high order cladding mode resonances. It can be clearly seen that for cladding mode resonances $30 \mathrm{~nm}$ away from Bragg resonance, triple pcaks appear

The TFBGs can be simulated by a commercially available optical fibre and waveguide grating simulation software: The OptiGrating [43] program, which is based on coupled-mode theory [20]. For a 3 layer step-index single mode fibre such as SMF-28, this software solves the LP modes analytically. The material dispersion is available for tilted gratings in this CAD tool, but it requires a very long simulation time. The TFBG simulation without including material dispersion shows large differences from the experimental result in the high order cladding mode (low wavelength) region. Therefore, for the TFBG the material dispersion must be included in the simulation for accurately analyzing the high order cladding mode resonance peaks. The simulation and experiment results are shown in Fig. 5.4. From Fig. 5.4, we can sec that if the material dispersion is not including in the simulation, the high order cladding mode resonances show a large "walk off" with the experimental result. By including the material dispersicn, the 
simulation result shows perfect matches for most of the cladding mode resonance peaks except the double peaked ones. As the LP modes can not simulate the double and triple peaked cladding mode resonances, the hybrid modes must be used.

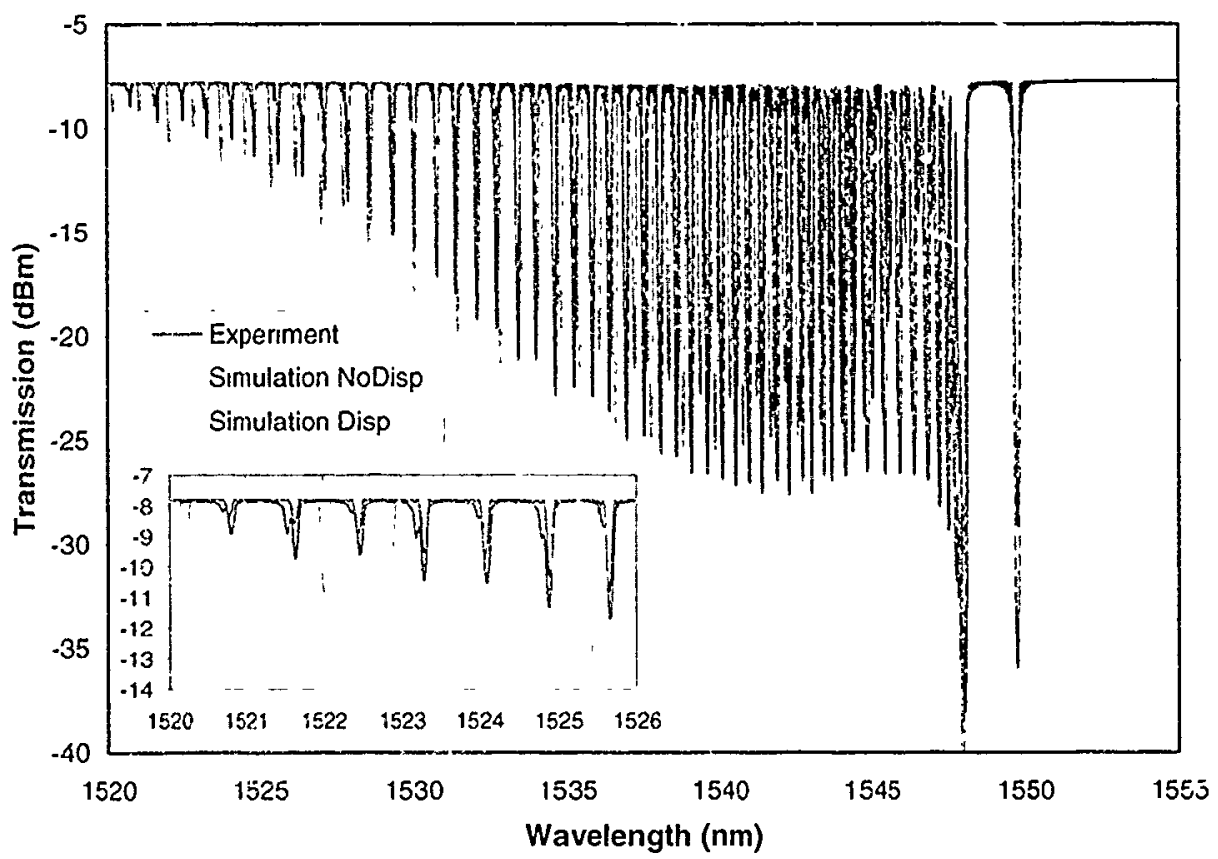

Figure 5.4: The experimental and simulated transmission spectrum of $4^{\circ}$ tilted FBG

Fig. 5.5 shows the simulation result obtained by using hybrid modes and the comparison with the experiment result. From Fig. 5.5, it can be seen that hybrid cladding mode resonances do show the same double peaked structure as the experimental ones. However, the double peaked resonance amplitudes for individual peaks are not exactly matched with experimental ones. This could be caused by the following facts: (i) the corc and cladding may not be perfect circular as the simulation assumed, (ii) having cladding modes with different azimuthal mode number $v(v=1,2,3 \cdots)$, (iii) the effect of ignoring 
the longitudinal coupling coefficients [22]. The experiment also shows that the dual peaked resonance amplitudes for individual peaks are very sensitive to small perturbations (temperature, strain and surrounding refractive index).

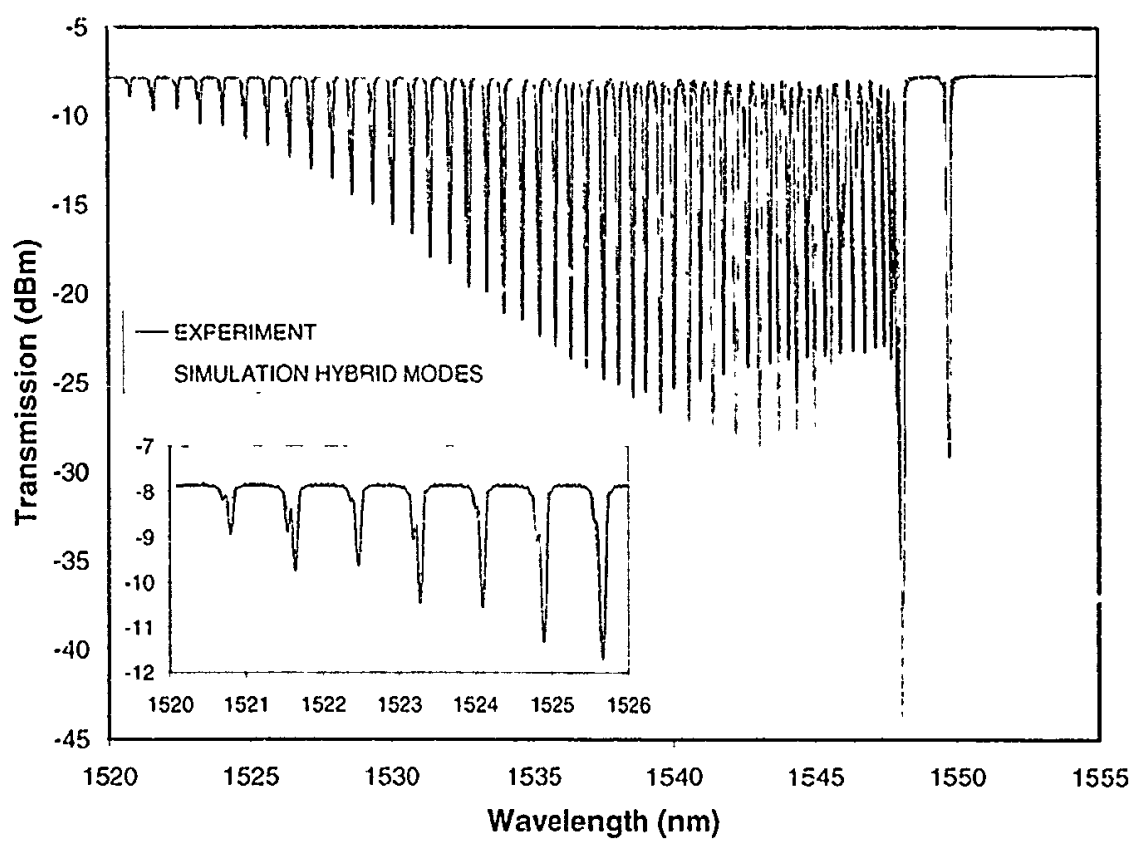

Figure 5.5: The simulated transmission spectrum by using hybrid modes and the experimental transmission spectrum of $4^{\circ}$ tilted FBG

Therefore, the LP modes can be used to simulate the coupling between the core mode and cladding modes accurately within a $20 \mathrm{~nm}$ span from the Bragg resonance. More than $20 \mathrm{~nm}$ away from the Bragg resonance the hybrid modes need to be used to an.lyze the cladding mode resonances of TFBGis. Double peaked cladding mode resonances can be simulated with hybrid modes and results are well matched with the experiment except for the individual peak amplitudes. When analyzins the high order cladding mode resonances of TFBG, the material dispersion effect can not be ignored and must be included in simulations. By accurately analyzing the higher order cladding mode 
resonances with double or triple peaks, the tracking of higher order cladding mode resonances is facilitated, and these modes have the largest differential strain and index sensitivities relative to the core modes. A better understanding of these higher order mode resonances will extend the range and sensitivity of strain and refractive index measurements by helping to accurately track those cladding mode resonance peaks.

\subsubsection{Polarization Dependence of the SMF-28 Mode Resonances}

The tilted angle in fibre core of TFBG makes it inevitably strongly polarization dependent. The doped core exposure to the excimer laser light when TFBGs are written also increase the non-uniformity in the cross-section of the core area. Fig. 5.6 shows the

L of the core and cladding mode resonances. It can be seen from Fig. 5.6 that the PDL of each mode is dependent not only on the resonance peak amplitude, but the position as well, for example the ghost mode has the largest resonance amplitude but not the largest PDL. The TFBG written by the $193 \mathrm{~nm}$ ArF excimer laser light has much larger PDL than the one written by $248 \mathrm{~nm} \mathrm{KrF}$ excimer laser light. The PDL difference could be caused by an unknown difference in the photosensitive mechanisms for the two types of irradiation. Fig. 5.7 further demonstrates the core and cladding modes polarization dependence characteristics but measure the transmission spectra of TFBG in SMF-28 fibres with different polarization states, where $\mathrm{S} 1$ is circular polarization, $\mathrm{S} 2$ is $0^{\circ}$ linear polarization, $\mathrm{S} 3$ is $90^{\circ}$ linear polarization and the $\mathrm{S} 4$ is $-45^{\circ}$ linear polarization. From the insets of Fig. 5.7, the core mode, the ghost mode and low order cladding mode do not show any strong polarization state dependence, because their fields are more confined in the fibre core region: most of the light is confined in a small area. For high order cladding 
modes, most of the light is confined in an area with a large radius, and much more in the vicinity of the outer cladding layer and surrounding media comparing with the low order cladding modes. Therefore the high order cladding modes are more sensitive to the cladding diameter uniformity as well as to any perturbations whe the measurement is going on such as temperature and vibration perturbations. The JDSU SWS-OMNI-2 swept wavelength system that is used for measuring transmission spectra has a resolution of $\pm 3 \mathrm{pm}$. It takes several minutes to do a full pelarization state measurement, and any perturbations in this period will show in transmission spectra.

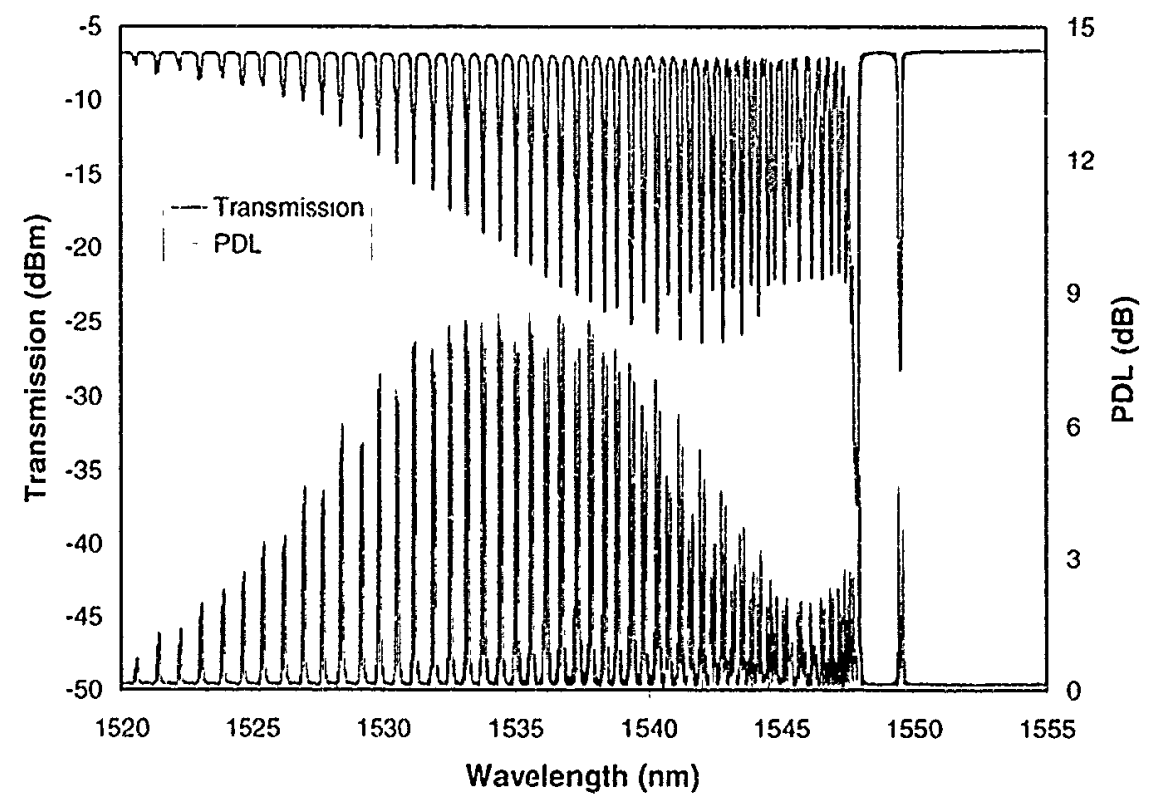

(a) 


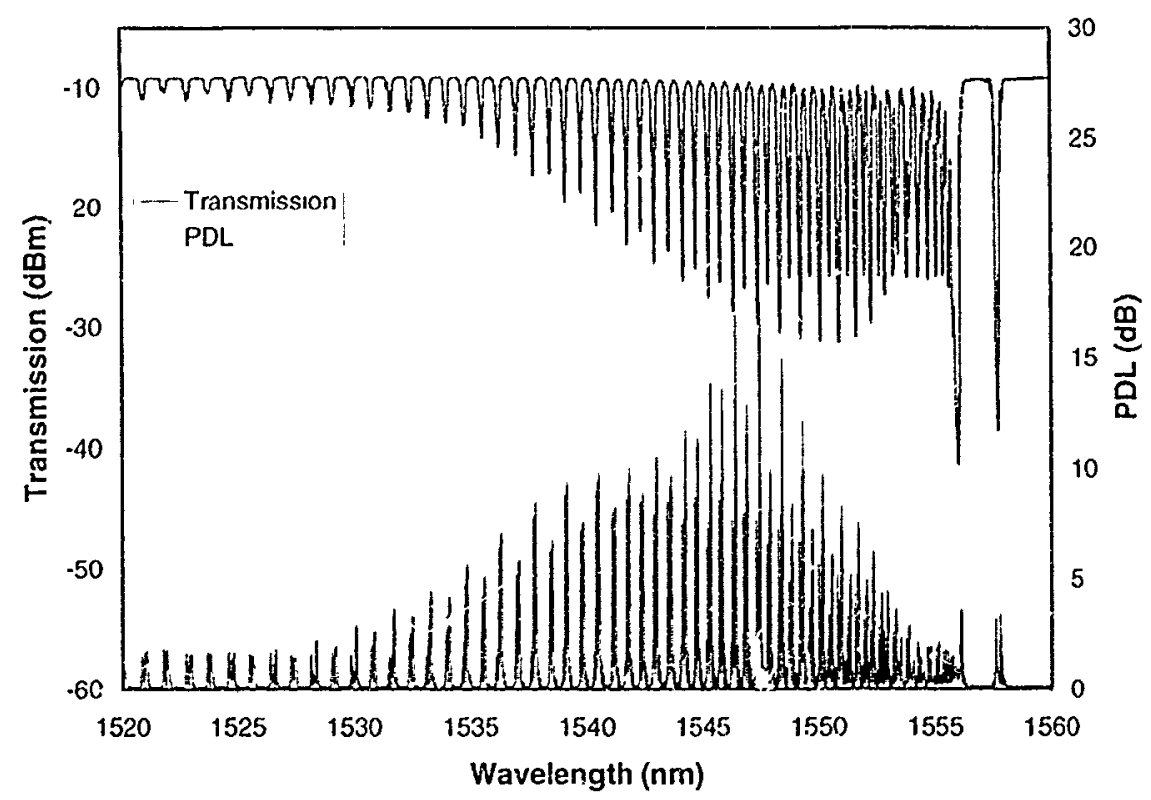

(b)

Figure 5.6: The PDLS of core and cladding modes: (a) TFBG written by $248 \mathrm{~nm} \mathrm{KrF}$ excimer laser light; (b) TFBG written by 193nm ArF excimer laser light.

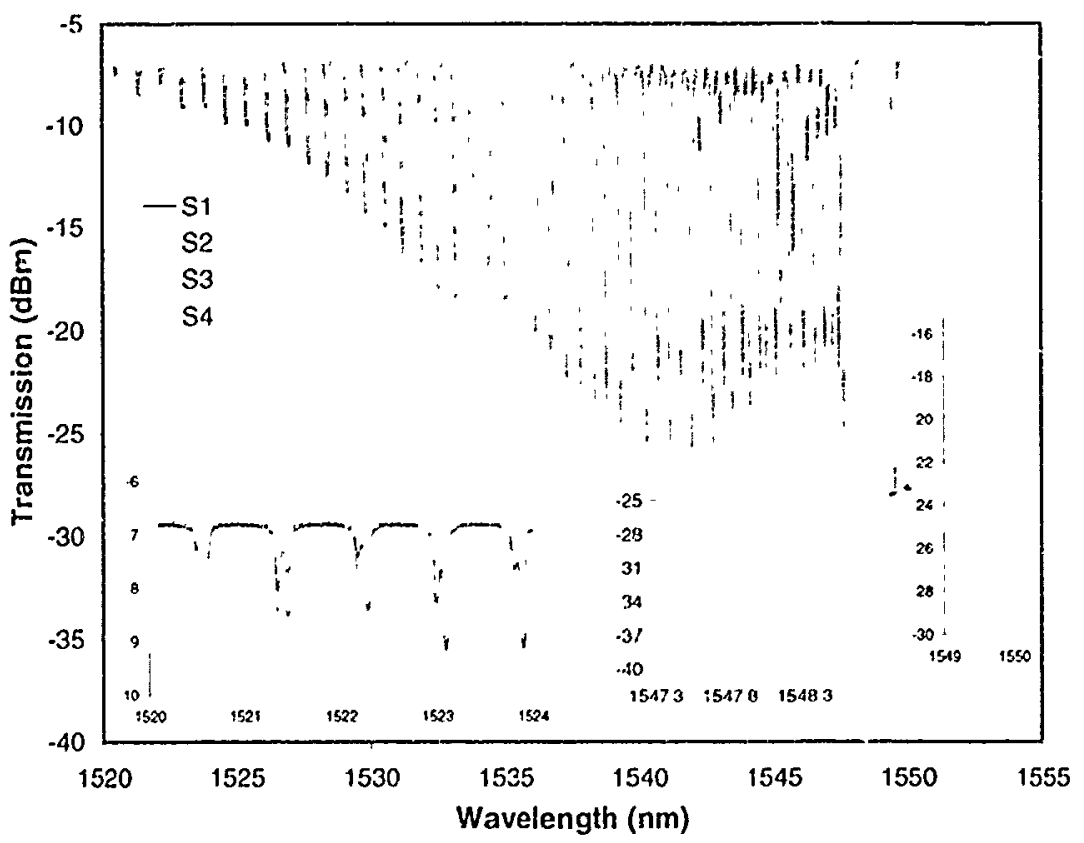

(a) 


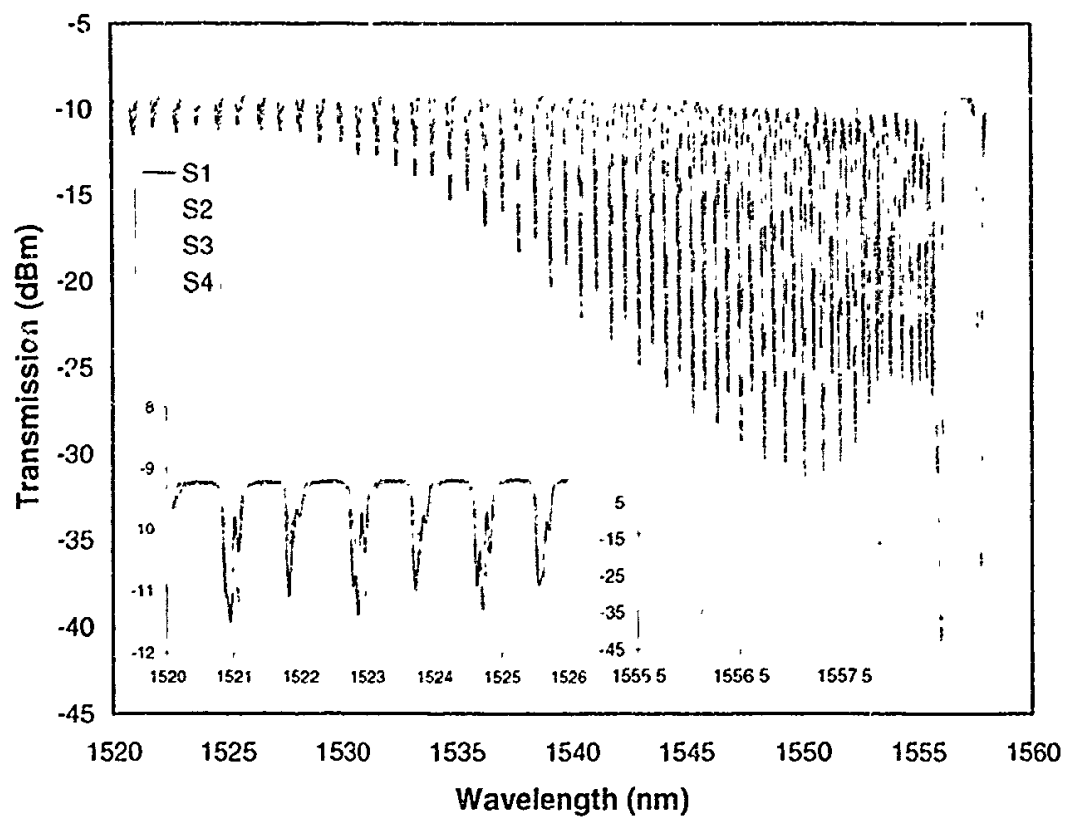

(b)

Figure 5.7: The Transmission Spectra of FEGs in PCF with Different Polarization States: (a) TFBG written by $248 \mathrm{~nm} \mathrm{KrF}$ excimer laser light; (b) TFBG written by $193 \mathrm{~nm}$ ArF excimer laser light.

\subsection{Analysis of Core and Cladding Mode Sensitivities of SMF-28 Fibres in TFBG with External Perturbations}

As is well known, the Bragg reflection and cladding mode resonance wavelengths $\lambda_{B}$ and $\lambda_{\text {ltal }}^{\prime}$ of TFBG are determined by a phase-matching condition and can be expressed as follows [51]:

$$
\begin{aligned}
& \lambda_{B}=2 n_{c f f}^{-} \Lambda / \cos \theta \\
& \lambda_{\text {clud }}^{i}=\left(n_{c f f}^{i}+n_{\text {clad }}^{i}\right) \Lambda / \cos \theta
\end{aligned}
$$


where $n_{e f f}, n_{e f f}^{i}$ and $n_{c t a d}^{i}$ are the effective indices of the core mode at $\lambda_{B}$ and the core mode and the ith cladding mode at $\lambda_{\text {clid }}^{\prime}$ respectively, and $\Lambda$ and $\theta$ are the period and the internal tilt angle of the TFBG.

\subsubsection{Strain Perturbations of TFBG}

For weakly TFBG, If we consider the wavelength shifts $\Delta \lambda_{B}$ and $\Delta \lambda_{\text {, lad }}^{i}$ caused by axial strain changes $(\Delta \varepsilon)$ only, the equations (5.1) and (5.2) can be written as follows [53]:

$$
\begin{aligned}
& \Delta \lambda_{B}=\left(\frac{2 n_{e f f}}{\cos \theta} \frac{\partial \Lambda}{\partial \varepsilon}+\frac{2 \Lambda}{\cos \theta} \frac{\partial n_{e f f}}{\partial \varepsilon}\right) \Delta \varepsilon \\
& \Delta \lambda_{\text {clud }}^{i}=\left(\frac{\left(n_{e f f, \text { core }}^{i}+n_{\text {eff }, \text { cladl }}^{i}\right)}{\cos \theta} \frac{\partial \Lambda}{\partial \varepsilon}+\frac{\Lambda}{\cos \theta} \frac{\partial\left(n_{\text {eff .core }}^{i}+n_{\text {eff .claul }}^{i}\right)}{\partial \varepsilon}\right) \Delta \varepsilon
\end{aligned}
$$

Eqs (5.3) and (5.4) can be written as follows [53]:

$$
\begin{aligned}
& \Delta \lambda_{B}=\lambda_{B}\left(1-p_{B}\right) \Delta \varepsilon \\
& \Delta \lambda_{\text {llad }}^{\prime}=\lambda_{\text {clad }}^{\prime}\left(1-p_{\text {cld }}^{\prime}\right) \Delta \varepsilon
\end{aligned}
$$

Where $p_{B}=-\frac{1}{n_{c f f}} \frac{\partial n_{e f f}}{\partial \varepsilon}$ and $p_{c l d}^{\prime}=-\frac{1}{n_{c f f}^{t}+n_{c l u d}^{\prime}} \frac{\partial\left(n_{e f f}^{i}+n_{c l a d}^{\prime}\right)}{\partial \varepsilon}$ are photoelastic coefficients for core (Bragg) mode and the ith cladding modes respectively, which may be calculated through core and cladding refractive index changes by

$$
\delta n=0.5 * n^{3}\left\lfloor p_{12}-v\left(p_{11}-p_{12}\right)\right] \delta \varepsilon
$$

where $p_{11}$ and $p_{12}$ represent the component of strain-optic tensor, $v$ is Poisson's ratio and $n$ is the core or cladding refractive index. The high order cladding modes have 
smaller effective indices and hence will have larger photoelastic coefficients than the core mode if the partial derivatives of all the effective indices with respect to a global strain change on the fibre are similar. From Eqs. (5.5) and (5.6) and noting that $\lambda_{B}>\lambda_{\text {ctad }}^{\prime}$ and $p_{B}<p_{c l u d}^{t}$ for high order cladding modes, we obtain:

$$
\Delta \lambda_{B}=\lambda_{B}\left(1-p_{B}\right) \Delta \varepsilon>\lambda_{\text {llau }}^{i}\left(1-p_{B}\right) \Delta \varepsilon>\lambda_{\text {clad }}^{\prime}\left(1-p_{\text {clud }}^{i}\right) \Delta \varepsilon=\Delta \lambda_{\text {laul }}^{i}
$$

So the Bragg peak should have a larger wavelength shift than the high order cladding mode resonances.

\subsubsection{Temperature Perturbations of TFBG}

If we consider the Bragg and cladding mode wavelength shifts $\Delta \hat{\lambda}_{B}$ and $\Delta \lambda_{\text {clud }}^{\prime}$ caused by temperature changes ( $\Delta T$ ) only, from Eqs. (5.1) and (5.2) the wavelength shifts $\Delta \lambda_{B}$ and $\Delta \lambda_{\text {clud }}^{\prime}$ can be written as follows:

$$
\begin{aligned}
& \Delta \lambda_{B}=\left(2 \frac{\Lambda}{\cos \theta} \frac{\partial n_{c f f}}{\partial T}+2 \frac{n_{e f f}}{\cos \theta} \frac{\partial \Lambda}{\partial T}\right) \Delta T \\
& \Delta \lambda_{\text {clad }}^{i}=\left(\frac{\Lambda}{\cos \theta} \frac{\partial\left(n_{c f f . c o r e}^{i}+n_{\text {eff .clad }}^{i}\right)}{\partial T}+\frac{n_{\text {eff .core }}^{i}+n_{c f f . c t a d}^{i}}{\cos \theta} \frac{\partial \Lambda}{\partial T}\right) \Delta T
\end{aligned}
$$

\subsubsection{Surrounding Refractive Index Perturbations of TFBG}

Consider the Bragg and cladding mode wavelength shifts $\left(\Delta \lambda_{B}\right.$ and $\left.\Delta \lambda_{\text {clad }}^{i}\right)$ caused by surrounding refractive index $\left(\Delta n_{s}\right)$ changes only, from Eqs. (5.1) and (5.2) the wavelength shifts $\Delta \lambda_{\beta}$ and $\Delta \lambda_{\text {llud }}^{i}$ can be written as follows: 


$$
\begin{aligned}
& \Delta \lambda_{B}=\left(2 \frac{\Lambda}{\cos \theta} \frac{\partial n_{e f f}}{\partial n_{s}}+2 \frac{n_{e f f}}{\cos \theta} \frac{\partial \Lambda}{\partial n_{s}}\right) \Delta n_{s} \\
& \Delta \lambda_{\text {slud }}^{i}=\left(\frac{\Lambda}{\cos \theta} \frac{\partial\left(n_{\text {eff.core }}^{i}+n_{\text {eff.clad }}^{i}\right)}{\partial n_{\mathrm{s}}}+\frac{n_{\text {c.f.core }}^{i}+n_{\text {eff.clud }}^{i}}{\cos \theta} \frac{\partial \Lambda}{\partial n_{s}}\right) \Delta n_{s}
\end{aligned}
$$

Due to $\partial \Lambda / \partial n_{s}=0$, and noting that $\partial n_{e f f} / \partial n_{s}=0$ and $\partial n_{e f f}^{2} / \partial n_{s}=0$ for the standard single mode telecommunication fibres such as CORNING SMF-28 fibres, we obtain:

$$
\begin{aligned}
& \Delta \lambda_{b}=0 \\
& \Delta \lambda_{\text {clad }}^{i}=\frac{\Lambda}{\cos \theta} \frac{\partial n_{\text {eff.clad }}^{i}}{\partial n_{s}} \Delta n_{s}
\end{aligned}
$$

This gives out the obvious result that only the cladding mode resonance wavelength shifts depend on the surrounding refractive index perturbations and that this dependence is scaled by the dispersion of the cladding mode effective index with the outside index.

Therefore the Bragg and cladding mode wavelength shifts $\left(\Delta \lambda_{B}\right.$ and $\left.\Delta \lambda_{\text {clat }}^{\prime}\right)$ caused by strain, temperature and SRI perturbations can be written as follows:

$$
\begin{aligned}
\Delta \lambda_{B}= & \left(\frac{2 n_{c f f}}{\cos \theta} \frac{\partial \Lambda}{\partial \varepsilon}+\frac{2 \Lambda}{\cos \theta} \frac{\partial n_{c f f}}{\partial \varepsilon}\right) \Delta \varepsilon+\left(2 \frac{\Lambda}{\cos \theta} \frac{\partial n_{c f f}}{\partial T}+2 \frac{n_{e f f}}{\cos \theta} \frac{\partial \Lambda}{\partial T}\right) \Delta T \\
\Delta \lambda_{\text {clad }}^{i} & =\left(\frac{\left(n_{c f f}^{i}+n_{c l a d}^{i}\right)}{\cos \theta} \frac{\partial \Lambda}{\partial \varepsilon}+\frac{\Lambda}{\cos \theta} \frac{\partial\left(n_{c f f}^{i}+n_{c l u d}^{i}\right)}{\partial \varepsilon}\right) \Delta \varepsilon \\
& +\left(\frac{\Lambda}{\cos \theta} \frac{\partial\left(n_{c f f}^{i}+n_{\text {clad }}^{i}\right)}{\partial T}+\frac{n_{c f f}^{i}+n_{\text {clud }}^{i}}{\cos \theta} \frac{\partial \Lambda}{\partial T}\right) \Delta T+\frac{\Lambda}{\cos \theta} \frac{\partial n_{c f f . l u d}^{i}}{\partial n_{s}} \Delta n_{s}
\end{aligned}
$$




\subsection{The Sensitivity of the Core and Cladding Modes of SMF-28 Fibres}

\subsubsection{Differential Strain Sensitivity of TFBG}

The characterization of the strain sensitivity of individual resonances was carriec! out by streiching a grating rigidly bonded to two micrometric translation stages. The distance between the stages is $65-78 \mathrm{~cm}$. The resolution of the fiber length stretch is $2.45 \times 10^{-3} \mathrm{~cm}$ which corresponds to the 29-38 $\mu \varepsilon$. Fig. 5.8 shows the relative wavelength shifts of cladding resonances with respect to the Bragg resonance when longitudinal strain is applite to a TFBG in standard SMF-28 fibre with cladding diarneter $\mathrm{d}=125 \mu \mathrm{m}$ and $\theta=4^{\circ}$ (a) written by $193 \mathrm{~nm}$ excimer laser; and (b) written by $248 \mathrm{~nm}$ excimer laser light. It can be seen for the strain perturbations that there

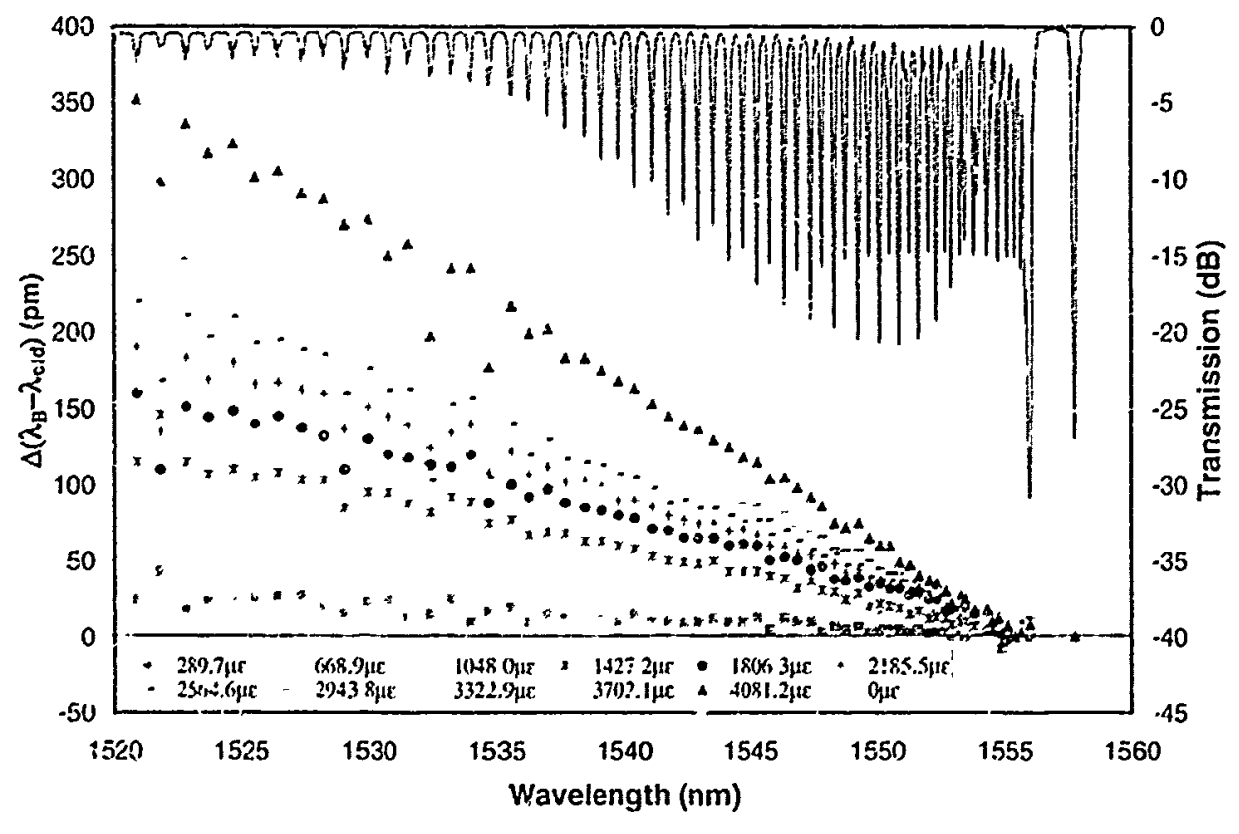

(a) 


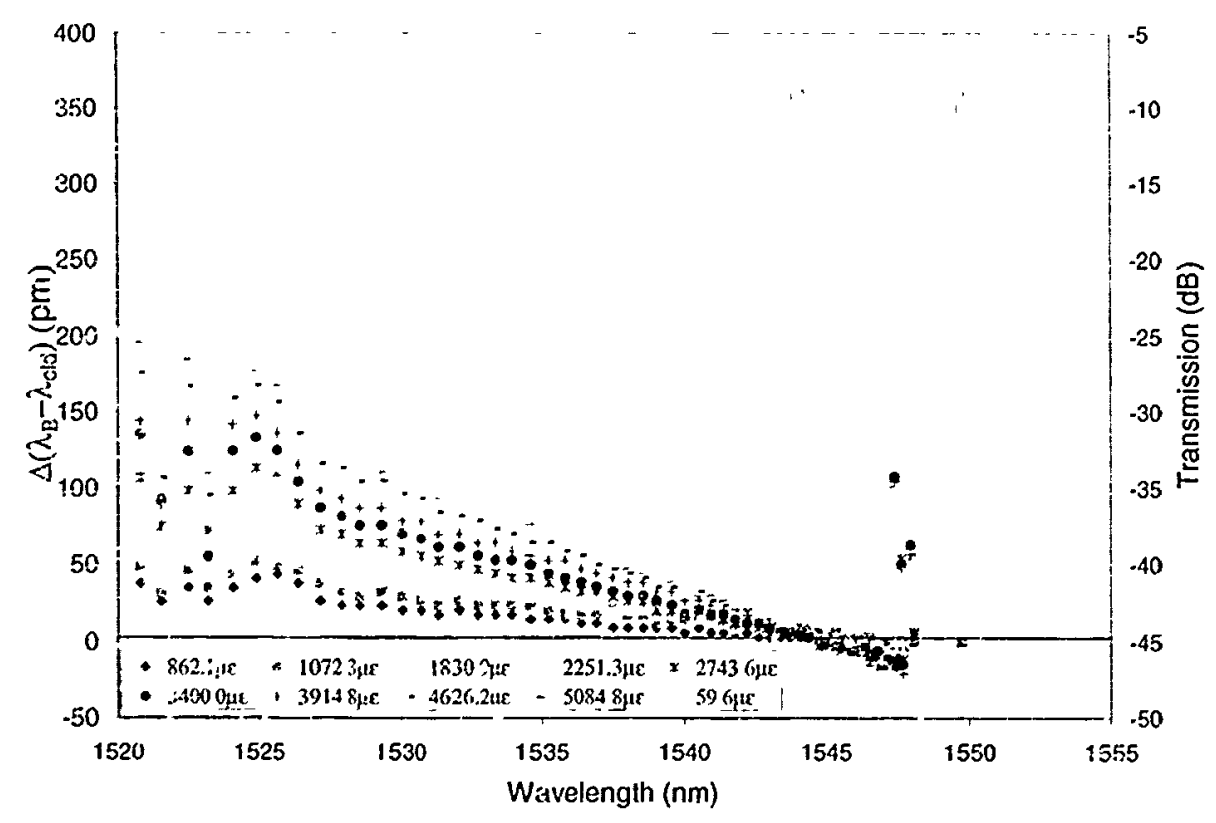

(b)

Figure 5.8: The transmission spectrum of $4^{\circ}$ tilted FBG and differential wavelength shifts due to strain changes: (a) written by $193 \mathrm{~nm}$ ArF excimer laser light; (b) written by $248 \mathrm{~nm}$ KrF excimer laser light.

are 3 differential wavelength shift regions. First, the ghost mode region appears to be sensitive to external strain perturbations with some low order cladding modes having negative relative wavelength shifts (i.e. cladding modes shift more than th.s core mode). For higher order modes, further than $20 \mathrm{~nm}$ from the Bragg resonance, the results become irregular because the cladding mode resonances have double- and triple-peaked resonances which are difficult to follow reliably over large shifting ranges. If the same individual peak of a multi-peak resonance could be followed unambiguously, then it is likely that the differential wavelength shift should continue to be linear in that region of the spectrum. Finally, between $5 \mathrm{~nm}$ and $20 \mathrm{~nm}$ from the Bragg resonance there is a wavelength region where the differential wavelength shift grows very linearly with mode 
order and with strain. This region of the spectrum will be the most useful for temperature independent strain sensors.

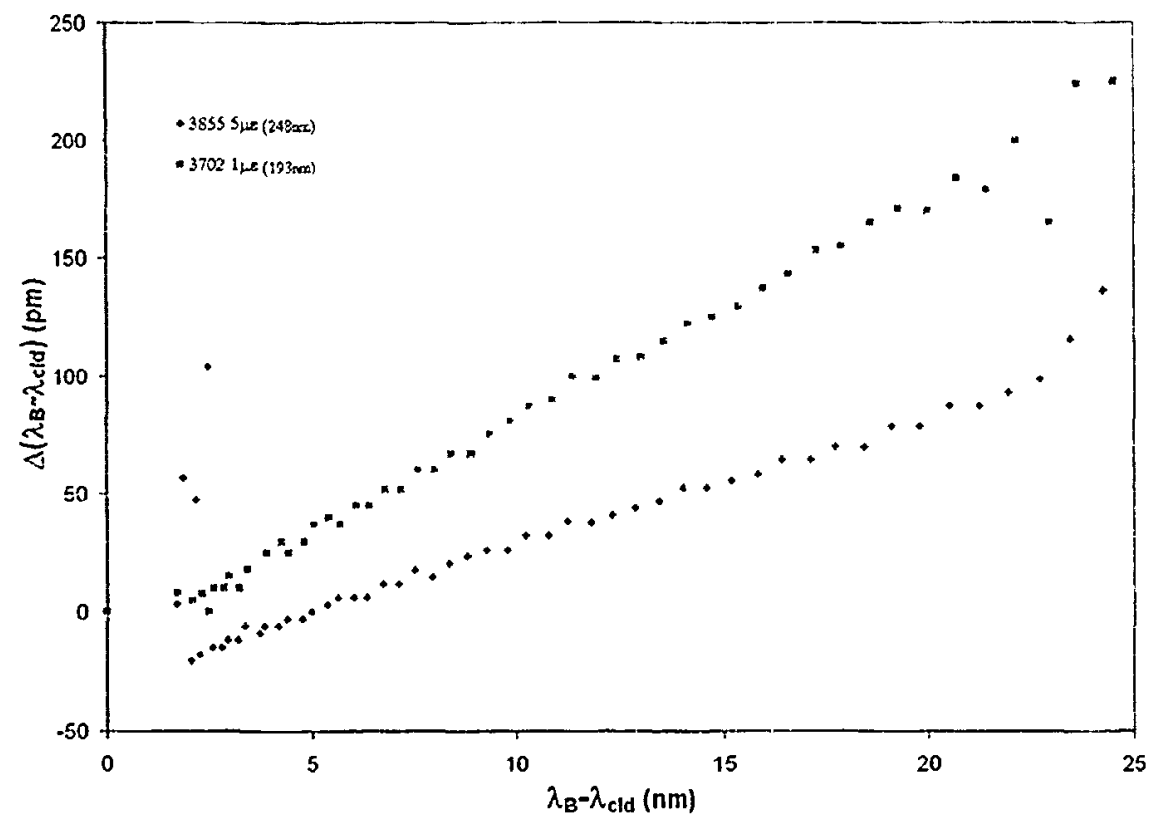

Figure 5.9: Differential wavelength shift of individual resonances for $4^{\circ}$ tilted FBCis written by ArF and KrF excimer laser light

Fig. 5.9 gives the comparison of the differential strain sensitivity of two $4^{\circ}$ tilted FBG sensors written in SMF-28 single mode fibre by $193 \mathrm{~nm}$ ArF excimer and by $248 \mathrm{~nm} \mathrm{KrF}$ excimer laser light respectively for a high strain value in each case (3702 $\mu \varepsilon$ and 3855 $\mu \varepsilon)$. Apart from irregular regions at both ends of the mode spectra in each case, the TFBG written at $193 \mathrm{~nm}$ shows a much larger differential strain. This result points to a yet unknown difference in the photosensitive mechanisms for the two types of irradiation. One hypothesis is that it has been shown previously that $193 \mathrm{~nm}$ irradiation of similar fibres to those used in this study causes significant compaction of the glass (more so than 
using $248 \mathrm{~nm}$ irradiation). This change in the physical structure of the glass could be sufficient to modify the photo-elastic coefficients $p_{11}$ and $p_{12}$ and hence the differential strain sensitivity of the TFBGs [54].

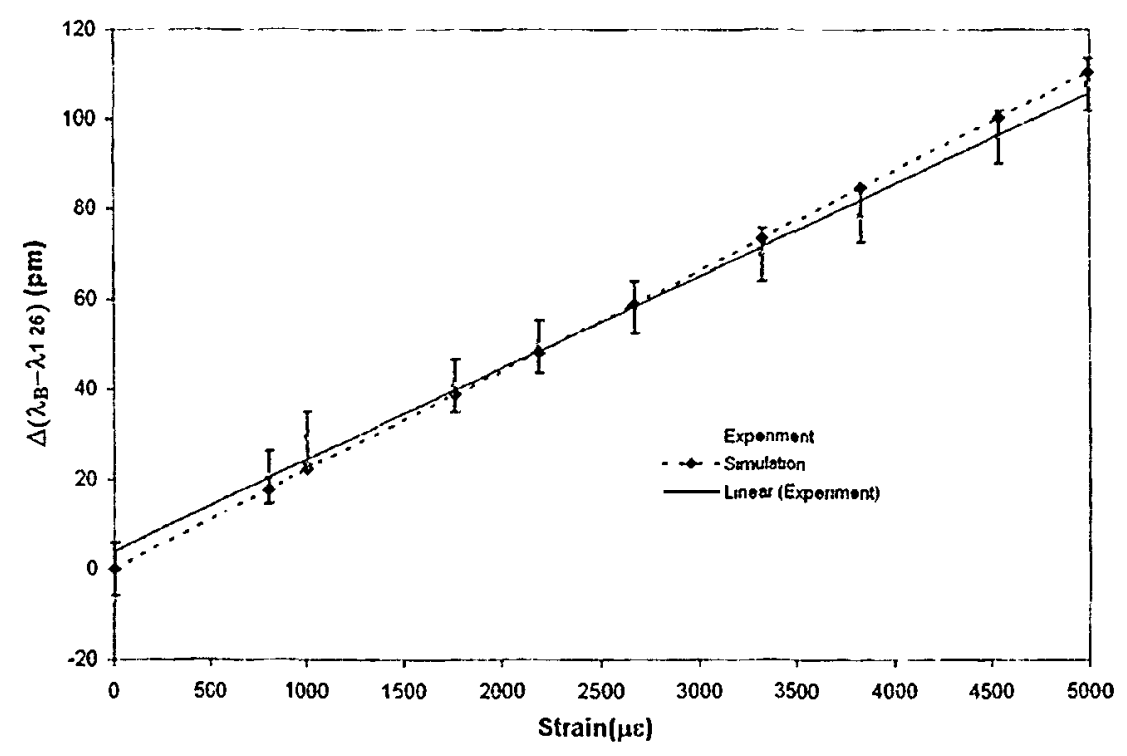

Figure 5.10: The relative wavelength shift of LP1 26 mode to LPO 1 mode for different strains with maximum temperature error

Fig. 5.10 shows the experimental and analytical results for the differential wavelength shift of mode $L P_{126}$ which has a resonance wavelength $\sim 1530 \mathrm{~nm}$ relative to the $L P_{01}$ resonance as a function of strain corresponding to Fig. 5.8(b). The error bars on the experimental result reflect the maximum temperature error $\sim \pm 6 \mathrm{pm}$ for $\sim 80^{\circ} \mathrm{C}$ temperature change that is shserved in the wavelength shift region that shows regular behaviour. The analytical result is obtained by Eqs. (5.5) and (5.6), where the partial derivatives of all the effective indices with respect to strain have been replaced by the value for bulk silica, obtained from Eq. (5.7) with $p_{11}=0.121$ and $p_{12}=0.27$, and Poisson's ratic $v=0.1720$. It 
can be seen that even with this approximation, the analytical and experimental results are in a good agreement (the small remaining difference reflecting the effect of the doping materials and waveguide dispersion on the partial derivatives making up Eqs. (5.5) and (5.6)).

\subsubsection{Differential Temperature Sensitivity of TFBG}

We now demonstrats: experimentally that the temperature sensitivity can be factored out of the measurements by using the differential wavelength shift (with the Bragg resonance as reference). The temperature sensitivity of the differential wavelength shift of cladding modes relative to the core mode wavelength shift is shown in Fig. 5.11 for the same TFBGs shown in Fig. 5.8. The temperature measurement was in a temperature-controlled o\%en which has a resolution of $0.1{ }^{\circ} \mathrm{C}$. To reduce the oven fan effect in gratings and increase the temperature uniformity, the fiber with TFBG was within a rectangular aluminum tube. The experimental results show that within the temperature range from $10^{\circ} \mathrm{C}$ to $70^{\circ} \mathrm{C}$, the relative cladding mode shifts are less than $\pm 12 \mathrm{pm}$ or equivalent to an apparent $\pm 1.2{ }^{\circ} \mathrm{C}$ drift in over $\sim 80{ }^{\circ} \mathrm{C}$ of temperature variation (except for the short wavelength region) for both TFBGs. Therefore, within this uncertainty and this temperature range at least, a temperature insensitive sensor can be made by monitoring the relative core and cladding mode wavelength shifts in the transmission spectrum. 


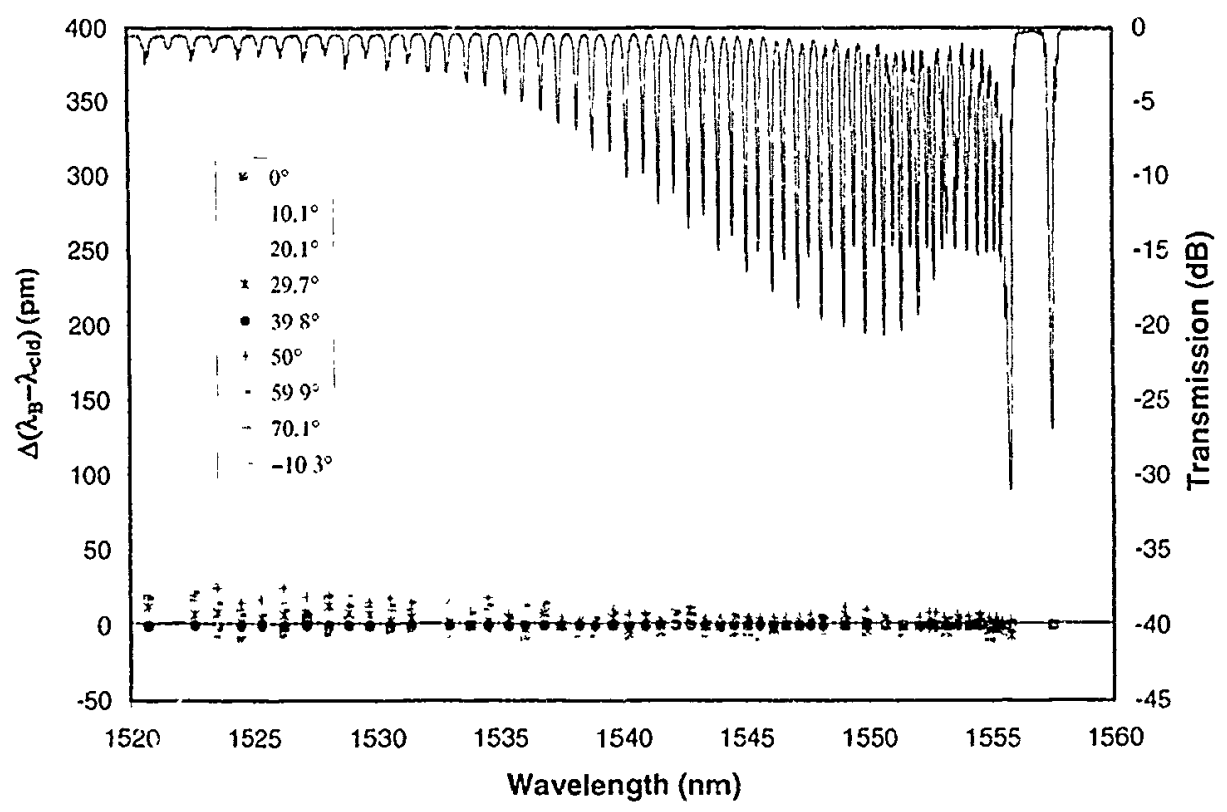

(a)

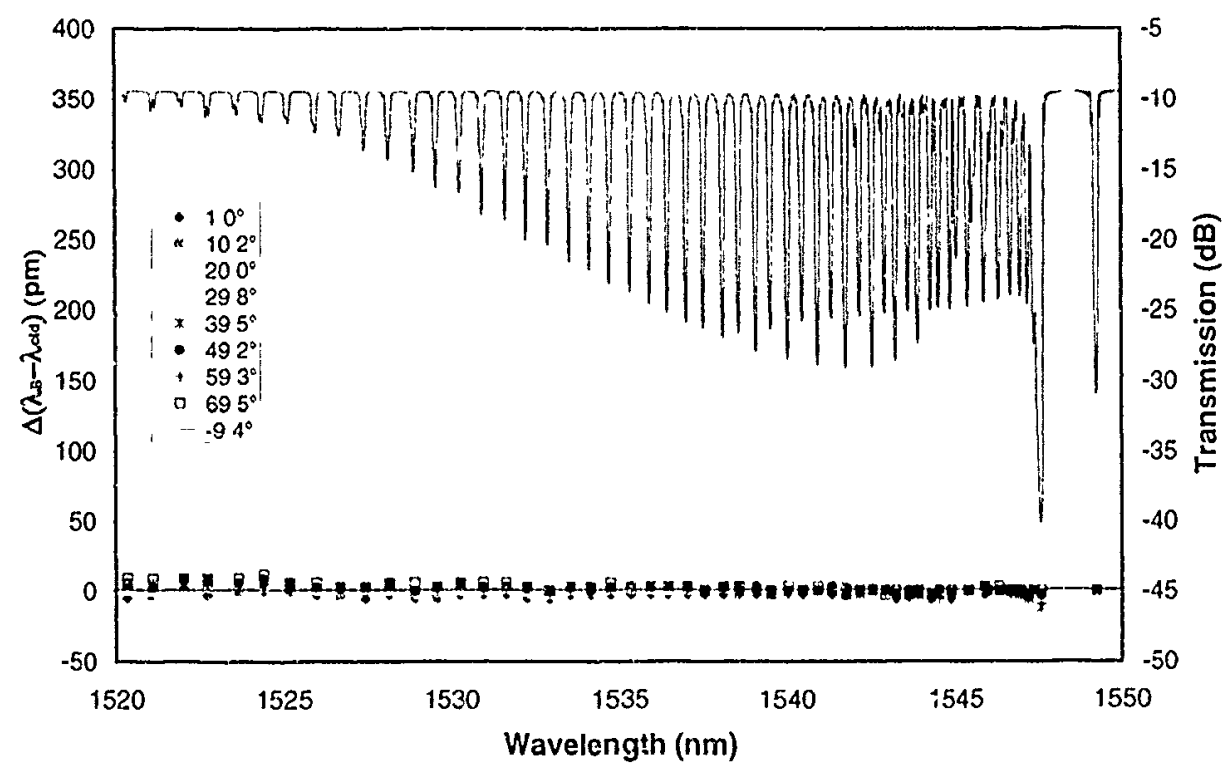

(b)

Figure 5.11: The transmission spectrum of $4^{n}$ tilted FBG and difierential wavelength shift due to the temperature changes: (a) written by 193nm ArF excimer laser light11; (b) written by $248 \mathrm{~nm} \mathrm{KrF}$ excimer laser light.

Fig. 5.12 shows how a single cladding resonance wavelength (near $1536 \mathrm{~nm}$ ) shifts with respect to strain and temperature. From the results, we can see that the differential 
wavelength shift increases almost linearly as the strain increases, while the differential wavelength shift due to the temperature changes is within $\pm 12 \mathrm{pm}$ in a range of $80^{\circ} \mathrm{C}$. The strain sensitivity of the differential wavelength shift is $49 \mathrm{~nm} /$ unit strain $(0.049$ $\mathrm{pm} / \mu \varepsilon$ ), while the temperature sensitivity of the same resonance is approximately 0.3 $\mathrm{pm} /{ }^{\circ} \mathrm{C}$ with some degree of randomness. If we interrogate this resonance without independent knowledge of temperature, the error due to temperature fluctuations corresponds to an uncertainty of $\pm 250 \mu \varepsilon$.

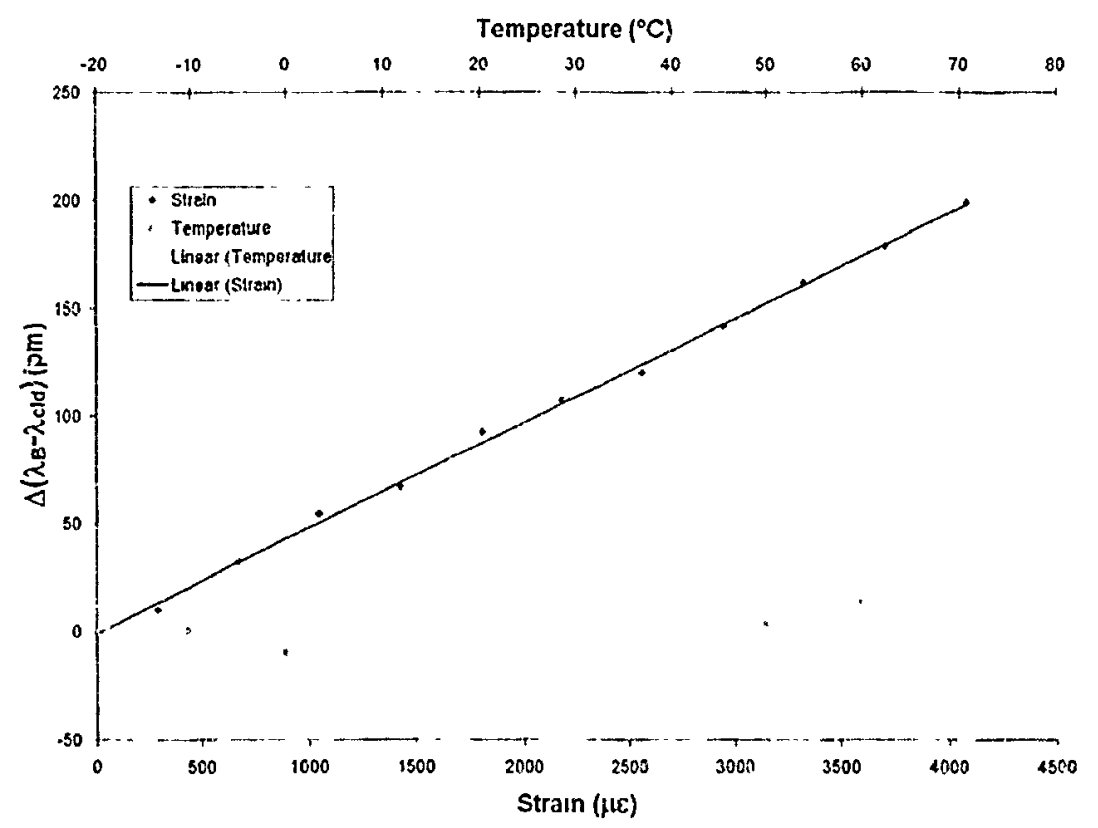

Figure 5.12: Comparison of the diffcrential wavelength shifts of a single cladding mode resonance for a $4^{\circ}$ tilted FBG at wavalength $\sim 1536 \mathrm{~nm}$ due to strain and temperature perturbations

\subsubsection{Surrounding Refractive Index Sensitivity}

The experimental TFBG used was written by $\mathrm{KrF}$ excimes laser light with $6^{\circ}$ tilt angle, its transmission spectrum measured in dir is shown in Fig. 5.13. The first set of results pertains to the measurement of sugar water solutions. The Fig. 5.14 (a) and (b) present 
the TFBG overlapped spectra for several valucs of $n_{1}$ (refractive index measured at 589 $\mathrm{nm}$ with an Abbe refractometer) ranging from 1.377 to 1.43 in sugar water solutions. There is a notable shift of the high order cladding medes while lower order modes and the Bragg resonance are not affected. The resonance shown in Fig. 5.14 (b) is located about $15 \mathrm{~nm}$ away from the Bragg resonance. It is clear that this cladding mode resonance shifts by amounts that are easily measurable (with respect to the width of the resonance, $\sim 100 \mathrm{pm}$ FWHM). This single resonance can be used to measure media with $n_{\text {D }}$ values between 1.0 and ahove 1.43 without ambiguity from neighbouring resonances since the spacing between resonance is larger than $600 \mathrm{pm}$ while the resonance shifts by $500 \mathrm{pm}$ over this range of $\mathrm{n}_{\mathrm{D}}$. If we now plot the relative resonance position (with respec, to $\lambda_{B}$ ) as a function of $n_{D}$ shown in Fig. 5.15, we see that there is a one-to-one relationship between SRI and resonance shift (another resonance located near $17 \mathrm{~nm}$ away from the Bragg resonance was used herc). A seventh-order polynomial fit of the experimental results can be used as a calibration to correlate the measured wavelength shift with $\mathrm{n}_{\mathrm{D}}$. However, the sensitivity is quite poor for low values of SRI $(20 \mathrm{pm} / \mathrm{RIU}$ near $n_{1}=1.38$ ) and increases dramatically as the SRI approaches the value at which this particular cladding mode becomes cut-off $\left(3900 \mathrm{pm} / \mathrm{RIU}\right.$ near $\left.\mathrm{n}_{\mathrm{D}}=1.43\right)$. This sensitivity 


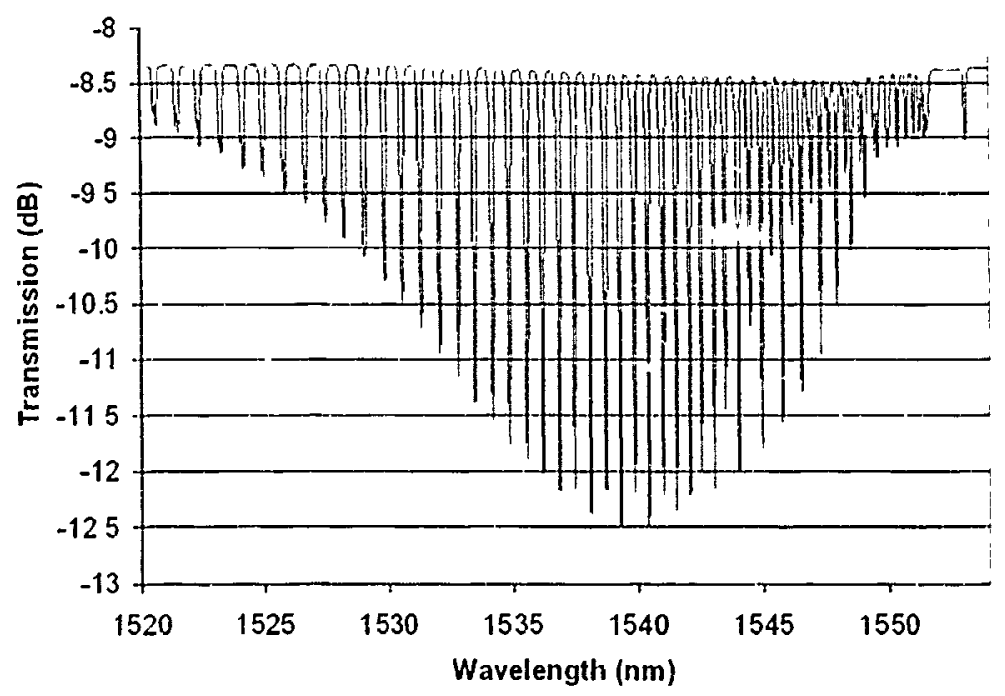

Figure 5.13: Typical experimental TFBG transmission spectrum (CORNING SMF28 fibre, $\left.\theta=6^{\circ}\right)$ measured in air.

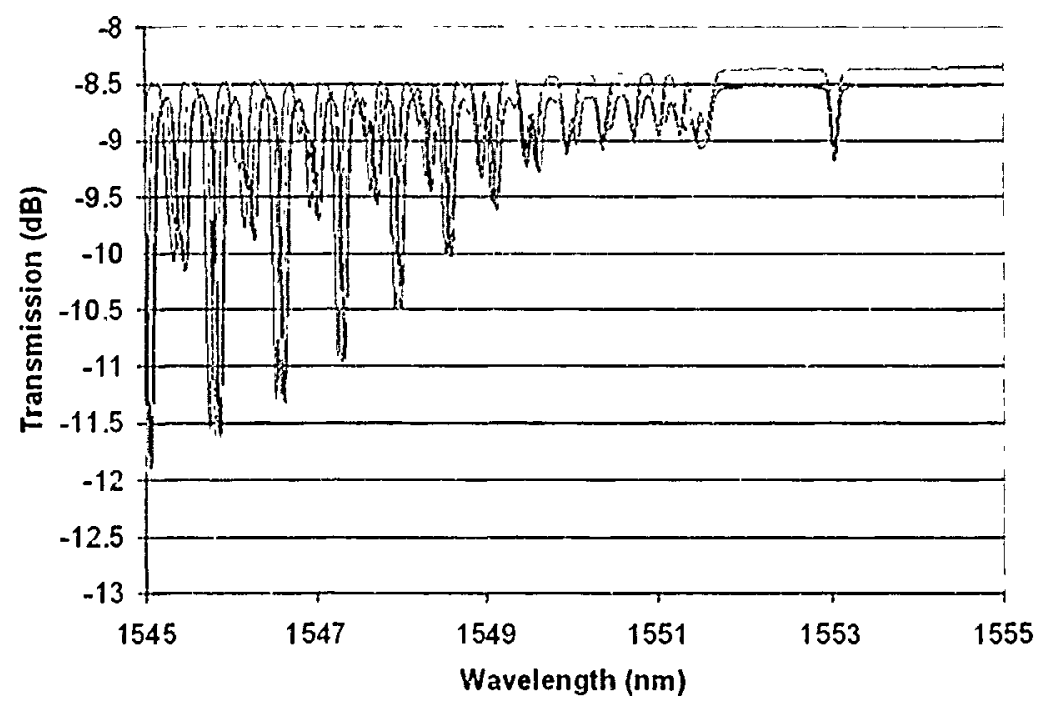

(a) 


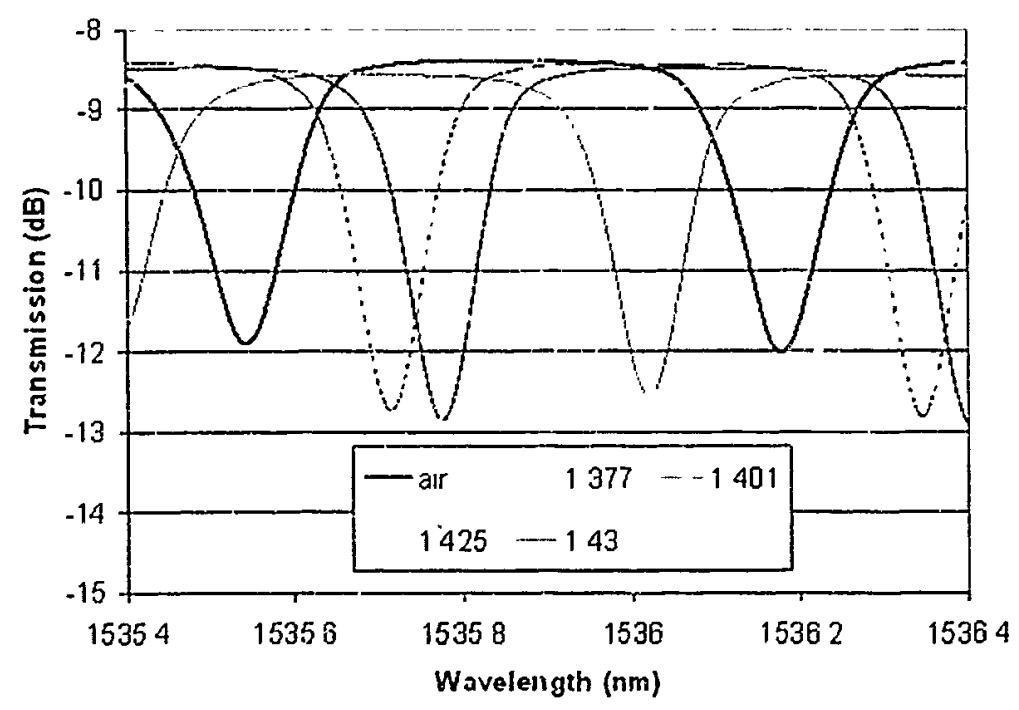

(b)

Figure 5.14: (a) Several measurements with various refractive indices of the outer medium, near the Bragg resonance. (b) Same spectra as (a) but zooming in on a particular resonance near $1535.5 \mathrm{~nm}$

increase near cut-off is typical of all sensiıg mechanisms that rely on the penetration of the evanescent field of the cladding modes into the outer medium [55]. However, this is where the TFBG configuration becomes advantageous since one can select different resonances of the same sensor, depending on the refractive index range of interest, to achieve the highest sensitivity. If the refractive index range of a particular situation is not known beforehand, then a simple pattern analysis of the transmission spectrum (compared to a stored transmission spectrum in air or in pure water) will reveal immediately which modes approach cut-off and where the measuring wavelength window should be located relative to the Bragg wavelength (for maximum sensitivity). By contrast, with an LPG-based sensor only a few (and generally only one or two) cladding modes are visible in a measurement window covering several hundred $\mathrm{nm}$. In 
such cases a new sensor design (grating period) is required to optimize the sensitivity over different refractive index regions.

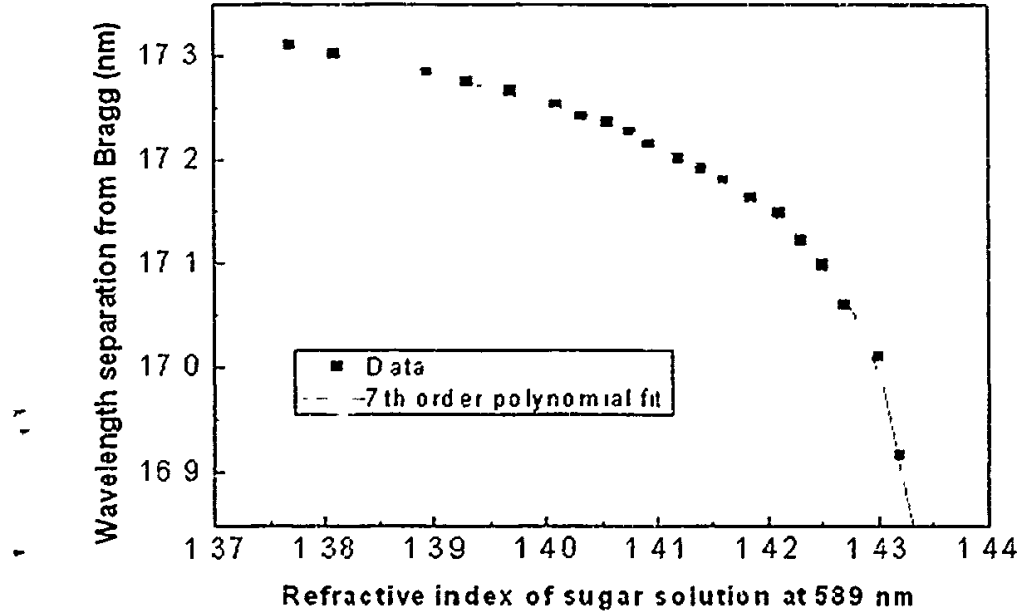

Figure 5.15: Experimental shift in the distance of a cladding mode resonance from the Bragg wavelength as a function of the refractive index of a sugar solution at $589 \mathbf{n m}$.

Further tests were carried out with calibrated liquids to demonstate how quantitativelỹ we can predict theoretically the refractive index sensitivity of several mode resonances and also to find out the temperature cross-sensitivity of our sensors. Fig. 5.16 shows the comparison between predicted and measured differential wavelength shifts as a function of surrounding refractive index for various cladding mode orders. The cladding modes are icientified here by the value of $\left(\lambda_{B}-\lambda_{C}\right)$ measured when the grating is in air (i.e. the original distance of the resonance from the Bragg wavelength irrespective of the mode identity and symmetry). The predicted shifts (solid lines) were obtained with d commercially available software package [43] using the refractive index profile and geometry of the standard fibre used. The fibre parameter used are: core radius $=4.15$ $\mu \mathrm{m}$, cladding radius $=62.5 \mu \mathrm{m}, \mathrm{n}_{\mathrm{corc}}=1.450699, \mathrm{n}_{\mathrm{clad}}=1.444024$, both at $1566 \mathrm{~nm}$ and 
corrected for dispersion for other wavelengths, using the dispersion of pure silica as a guide and offsetting the dispersion clirves to the refractive indices given ab $\lrcorner$ ve for $\lambda=$ $1566 \mathrm{~nm}$. When experimental error is taken into account all the measurements agree with values predicted from the model. For single measurements, we. use an error estimate of $+/-12 \mathrm{pm}$ for the exact value of the wavelength distance between a resonance and the Bragg wavelength relative to its value in air, to reflect an absolute uncertainty of at least $3 \mathrm{pm}$ on each wavelength measured. For some of the measurements shown in Fig. 1.16, five independent measurements were made with the same liquid, and the average value of the wavelength distance was calculated. In such cases the error estimate is provided by the standard deviation of the measurements. The standard deviation for these measurements never exceeded $3 \mathrm{pm}$. The uncertainty in the value of the refractive index is given as $+/-0.0002$ (at $589 \mathrm{~nm}$ ) by Cargille. For longer wavelengths the supplier provides Cauchy coefficients to calculate the dispersion of the refractive index but with an added uncertainty of the order of 0.005 . Our results seem to indicate that the actual uncertainty of the refractive index of the liquids is much smaller. 


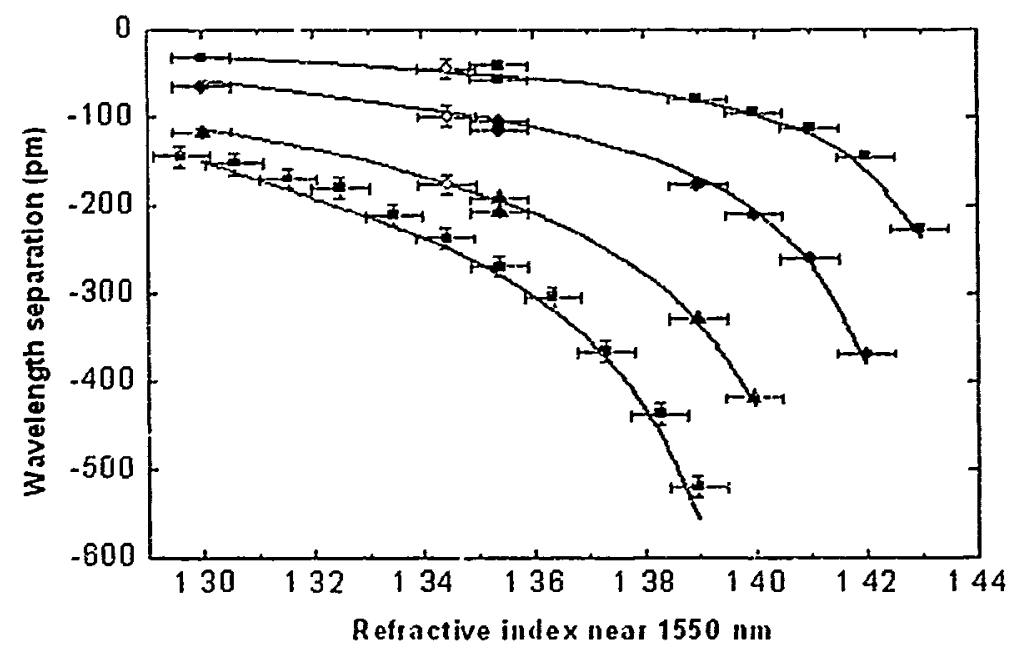

Figure 5.16: Change in wavelength separation from the Bragg wavelength for four different cladding modes as a function of the refractive index of calibrated liquids Filled symbols = experimental data at room temperature $\left(23^{\circ} \mathrm{C}\right)$; Open symbols = Experimental data at 50 ${ }^{\circ} \mathrm{C}$; Lines = simulations.

In order to validate our claims of relative temperature independence, an additional measurement was carried out at a temperature of $50{ }^{\circ} \mathrm{C}$ (open circles on Fig. 5.16). When the thermo-optic coefficient of the liquid $\left(-3.41 \times 10^{-3} /{ }^{\circ} \mathrm{C}\right)$ is taken into account, the refractive index values obtained from the grating also fall on the expected curve. This indicates that the sensor was able to measure the true refractive index of the liquids (within error), even though all its wavelength resonances were shifted by about $265 \mathrm{pm}$ due to the increase in temperature $\left(+27^{\wedge} \mathrm{C}\right)$. The sensitivity of the SRI measurements (and simulations) reaches over $10000 \mathrm{pm} /$ RIU for each of the resonances shown in Fig. 5.16 , corresponding to better than $10^{-4}$ refractive index sensitivity (RIU/pm) between 1.38 and 1.43 . The sensitivity calculated here for single resonance measurcments $\left(10^{-4} / \mathrm{pm}\right)$ obviously needs to be adjusted by the wavelength $\mathrm{m}^{\mathrm{r}}$ rement accuracy (about $10 \mathrm{pm}$ for the relative shift) to yield an actual sensitivity closer to $10^{-3}$ in SRI in our 
experiments. On the other hand, modern dedicated fibre grating interrogation equipment claims a wavelength measurement accuracy better than $1 \mathrm{pm}$ [56], making $10^{-4}$ sensitivity a definite possibility. With such an instrument, we have achieved a repeatability of the measurement of the wavelength distance (standard deviation of successive measurements of a fixed experimental situation) of $0.6 \mathrm{pm}$.

If there is a need for higher sensitivity at lower values of $n_{\mathrm{ext}}$, there are two design modifications that can be used to do so. First, it is possible to generate even higher order mode resonances with the TFBG, so that accurate resonance measurements can be made further away from the Bragg resonance. This can be achieved with a larger tilt angle, as shown in Fig. 5.17 where cladding mode resonances as far away as $100 \mathrm{~nm}$ from the Bragg wavelength are observed for tilt angles near 10 degrees. These resonances have effective indices near 1.25 and hence allow high sensitivity measurements in this range of values of the SRI. With the grating shown in Fig. 5.17, we have achieved $11200 \mathrm{pm} / \mathrm{RIU}$ for a SRI near 1.31 by using the resonance at $1500 \mathrm{~nm}$ (see Fig. 5.19). The additional benefit of using resonances further away from the Bragg wavelength is that their spacing increases (to over $1200 \mathrm{pm}$ in this case), thereby augmenting the range of unambiguous readings for the position of the resonance relative to its position in air (a similar effect occurs with reduced diameter cladding fibres [57] but require custom fabrication of the fibres or thinning methods lead to reduced mechanical strength). A second way to increase sensitivity in the lower index range is to use a thin layer of high index material on the cladding to increase the extent of the evanescent field of certain cladding modes into the outside medium. This may lead to an increased overlap and sensitivity to SRI 
changes, as was shown in the case of LPGs [58,59]. A major contrasting point between our TFBGs and devices based on LPGs, a large number of cladding modes is always available with a single grating device design, and that the exact wavelength of the Bragg resonance of the device is quite irrelevant (as well as the exact strength of the grating!). These relaxed tolerances ensure that the mass production of such devices at very low cost is definitely possible.

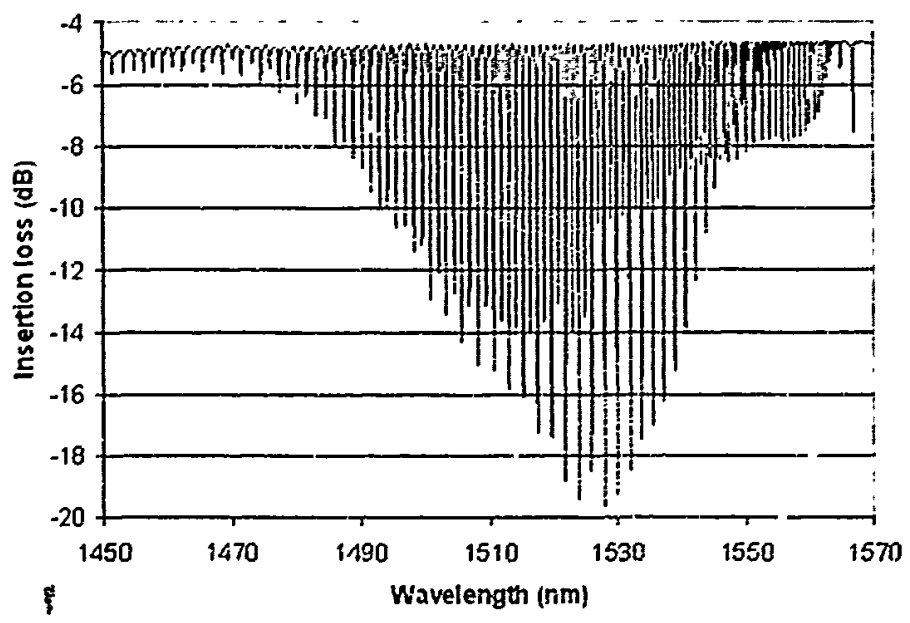

Figure 5.17: Experimental transmission spectrum of a TFBG with a tilt angle of 10 degrees, $\left(\lambda_{\mathrm{Bragg}}=1566.810 \mathrm{~nm}\right)$.

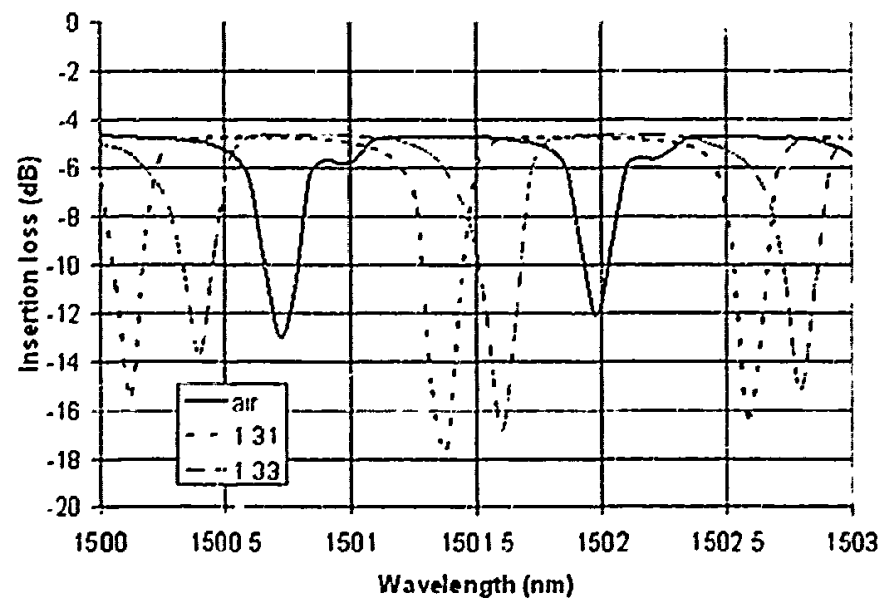

Figure 5.18: Shift of a resonance near $1500 \mathrm{~nm}$ for various external media (solid line: $S R I=1.0$, dotted line: $S R I=1.3058$, dashed line: $S R I=1.3250$, all values for $1500 \mathrm{~nm}$ )) 
Finally, the availability of several resonances within a single sensor measurement provides not only the possibility of multimodal sensing but also a way to increase the measurement accuracy of a given sensing mechanism by averaging the result obtained with the shifts of many cladding modes simultaneously. Since any systematic wavelength error is already eliminated by the fact that we are measuring relative wavelength shifts, the wavelength errors associated with each resonance should be statistically independent. Taking the results of Fig. 5.17 as an example, we have four independent wavelength shift measurements for each value of SRI. We can calculate a predicted SRI from the simulation for each of these measurements, take the average and plot the results against the actual SRI as calculated from the supplier data and taking into account dispersion. The result is shown in Fig. 5.19. In spite of the fact that the supplier only guarantees $+/-0.0052$ accuracy on the refractive index of the liquids for wavelengths away from $589 \mathrm{~nm}$, we obtain an excellent agreement between measured and expected values (as indicated by the slope of 0.999 and intercept of $5 \times 10^{-4}$ ) as well as a very good correlation $(\mathrm{R} 2=0.997)$. In fact, while the refractive index predictions from single measurements have errors ranging from -0.012 to +0.006 , the errors obtained by averaging the predictions from 4 resonances range only from -0.0009 to 0.001 . Even better results would be expected from a more sophisticated algorithm able to average the predictions of all the available resonances. 


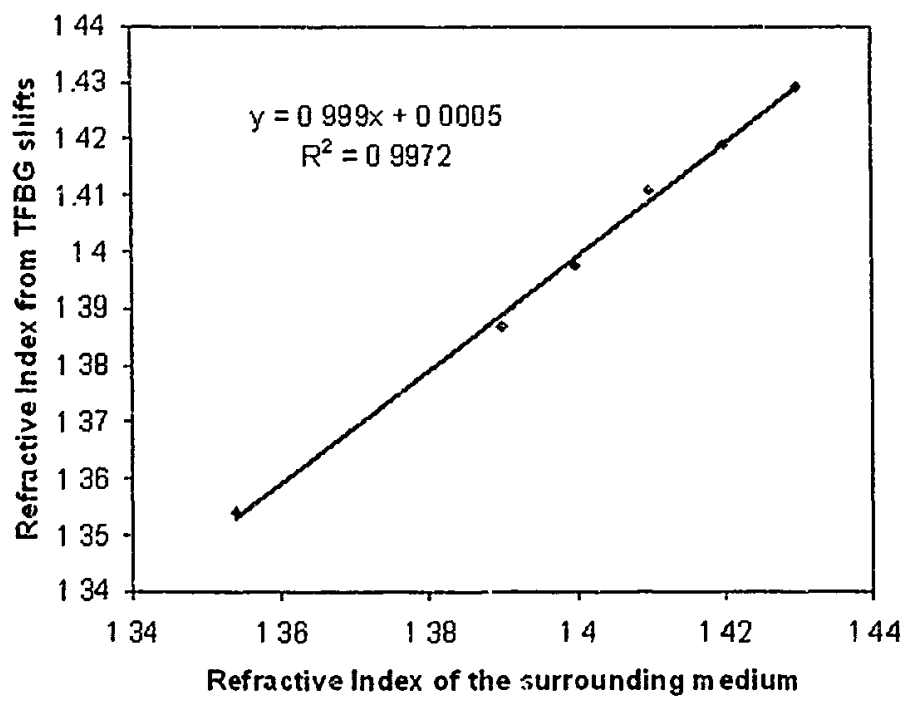

Figure 5.19: Measured refractive index from the average of the predictions based on the shifts of 4 resonances $(-8 \mathrm{~nm},-14 \mathrm{~nm},-23 \mathrm{~nm}$, and $-28 \mathrm{~nm})$ versus actual refractive index.

For comparisons, refractometers based on LPGs will have sensitivities of the order of $100-1000 \mathrm{~nm} / \mathrm{RIU}$ with cross sensitivity to temperature of the order of $1 \mathrm{~nm} /{ }^{\circ} \mathrm{C}$ and above for the absolute measurement of the wavelengths of resonances with bandwidths ranging from $10-100 \mathrm{~nm}$ [58-60]. In the best cases, the sensitivity divided by the resonance bandwidth is 100 (RIU) $^{-1}$ for LPGs, exactly equivalent to our results since it is just as easy to monitor pm shifts of $100 \mathrm{pm}$ resonances as it is to monitor $\mathrm{nm}$ shifts of $100 \mathrm{~nm}$ resonances (easier in a way because the required spectral range of the interrogation systems is much smaller) and the same sensitivity enhancement techniques [58-60] can be applied to TFBGs as tc LPGs. The only differences are that TFBGs have an inherent tempcrature monitoring channel (the Bragg resonance), and that they provide several measurements simultaneously to either achieve the highest sensitivity over several ranges of SRI, or to improve accuracy by averaging several measurements from a single sensor. 


\subsection{The Sensitivity of the Core and Cladding Modes of SMFs with Thinned Cladding Layer Thickness}

In order to enhance the external refractive index sensitivity of both FBG and LPG, thinned cladding layer thicknesses are preferred $[20,53]$. In the case of TFBGs, the effect of reducing the cladding layer thickness for an external refractive index sensor is shown to increase the sensitivity substantially $[10,11]$. In the work presented here, we report both experimental and analytical results on the differential strain and temperature sensitivitics of cladding and core mode resonances in fibres with reduccd cladding diameter. The fibres studied are a standard CORNING SMF-28 fibre etched to reduce its outer diameter to $50 \mu \mathrm{m}$, and a specially fabricated $50 \mu \mathrm{m}$ cladding layer diameter exr imental fibre (also from CORNING, and otherwise similar to SMF-28).

\subsubsection{Differential Strain and Temperature Sensitivities of TFBG}

The experimental TFBG sensors are all written in hydrogen-loaded CORNING SMF-28 fibres and CORNING thin cladding layer experimental fibre by a $248 \mathrm{~nm} \mathrm{KrF}$ excimer laser light and a phase mask to generate the grating pattern. Fig. 5.20 shows transmission spectra of three TFBG sensors: (a) the TFBG is written in a SMF-28 fibre with tilt angle $\theta=4^{\circ}$ (the same TFBG as shown in Fig. 5.8 (b)), (b) the TFBG is written in a CORNING experimental $50 \mu \mathrm{m}$ cladding layer diameter fibre with tilt angle $\theta=6^{\circ}$, and (c) the TFBG is first written in a SMF-28 fibre with tilt angle $\theta=4^{\circ}$, then etched in $50 \% \mathrm{HF}$ (water 


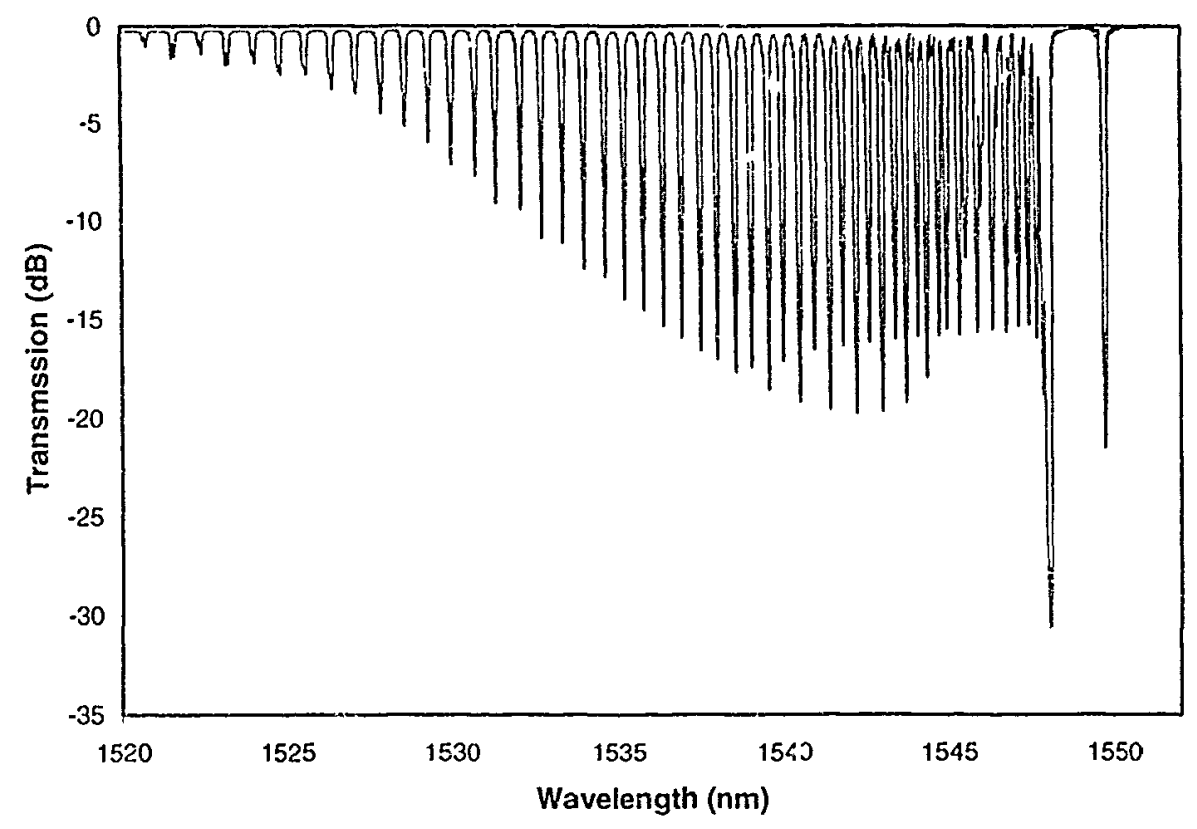

(a)

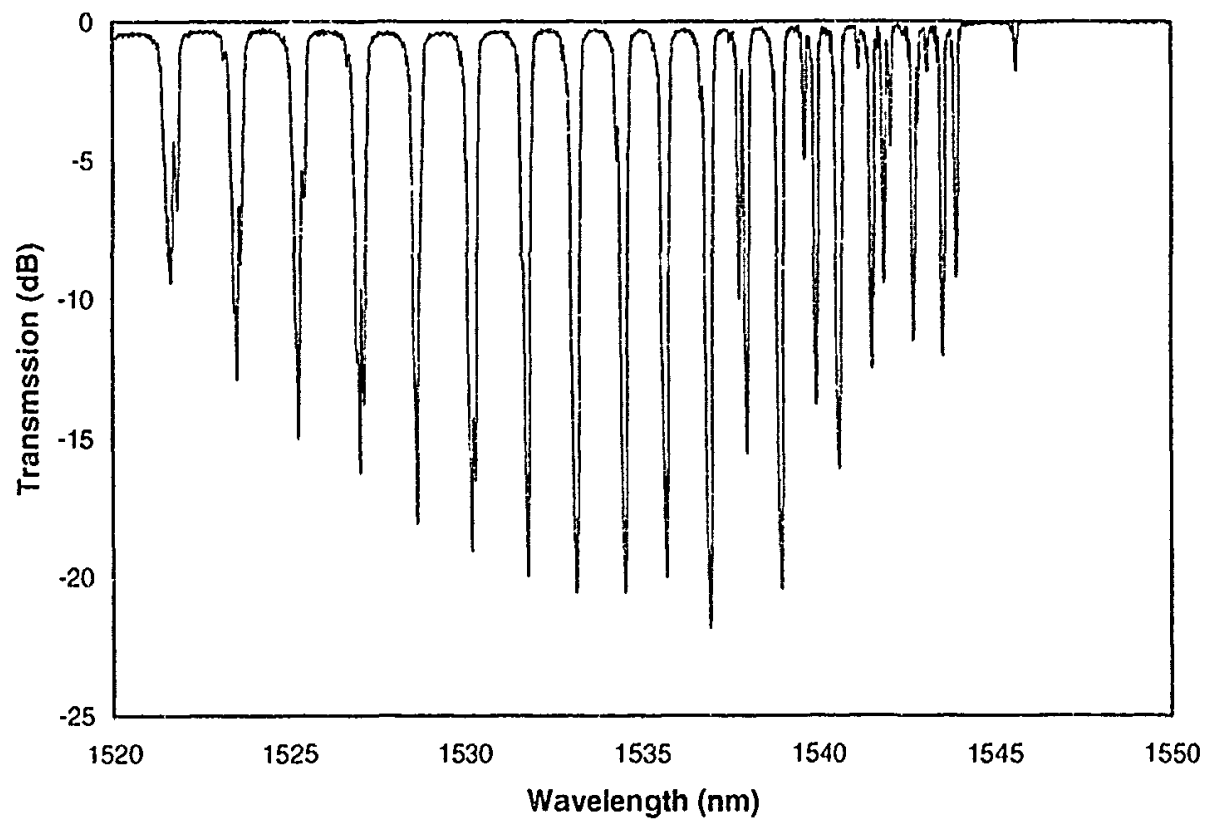

(b) 


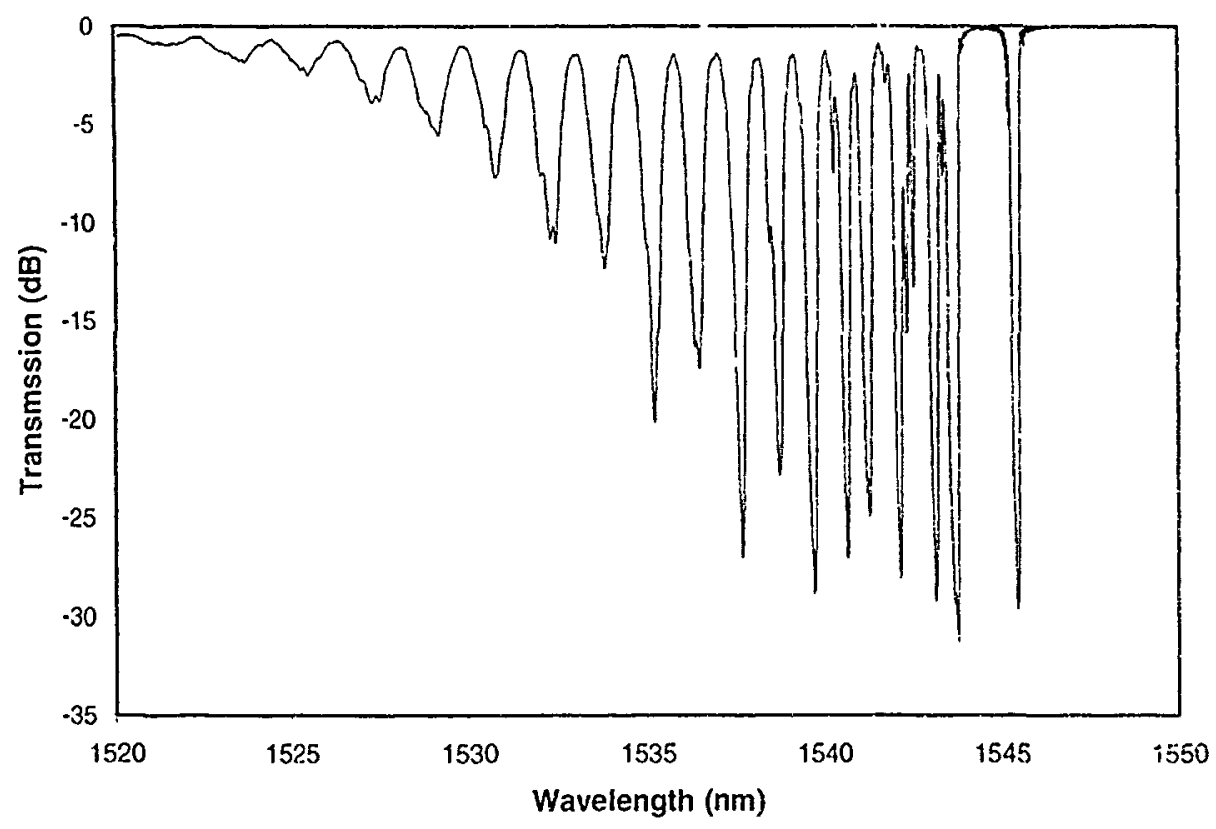

(c)

Figure 5.20: The transmission spectra of weakly TFBG: (a) SMF-28 fibre $d=125 \mu m$ and $\theta=4^{\circ}$; (b) Corning experimental thin cladding layer fibre $d=50 \mu \mathrm{m}$ and $\theta=6^{\circ}$; (c) Thin cladding layer by HF etching from SMF-28 fibre $d=50 \mu \mathrm{m}$ and $\theta=4^{\circ}$.

solution) to $50 \mu \mathrm{m}$ cladding layer diameter. It can be seen from Fig. 5.20 that the thinne1 cladding layer thickness results in fewer cladding modes that are more widely separaled in wavelength. This provides the added benefit that it becomes much easier to track individual resonances. One remarkable feature is that while cases b) and c) are nominally equivalent (apart from the tilt angle), the spectral quality of grating c) is much poorer (broader, poorly defined resonances, especially at shorter wavelengths). It is suspected that the poor spectral quality is caused by surface roughness remaining after the etching process, while fibre b) has the pristine surface of pulled fibre.

Fig. 5.21 (a) shows the relative wavelength shifts of cladding resonances with respect to the Bragg resonance when longitudinal strain and temperature perturbations are 
anplied to a TFBG in standard SMF-28 fibre with $d=125 \mu \mathrm{m}$ and $\theta=4^{\circ}$. It con be seen for the strain perturbation that there are 3 differential wavelength shift regions. First, within $5 \mathrm{~nm}$ from the Bragg resontance, the ghost mode appears to be very sensitive to external strain perturbations while other low order cladding modes have negative relative wavelength shifts. For higher order modes, further than $20 \mathrm{~nm}$ from the Bragg resonance, the results are quite irregular and there are several cladding modes with double peaked resonances which appear to have very large relative wavelength shifts. These irregular shifts may be due to improper peak tracking within the double peaks than to actual strain sensitivity. Finally, between $5 \mathrm{~nm}$ and $20 \mathrm{~mm}$ from the Bragg resonance there is at wavelength region where the difierential wavelength shift grows very linearly with mode order and with strain. The lower part of the graph shows that the relative wavilength shifts of cladding resonances to the Bragg resonance with temperature from $9.7^{\circ}$ to $59.5^{\circ}$ is very small, it is arounc $\pm 12 \mathrm{pm}$ corresponding to $0.35 \mathrm{pm} /{ }^{\circ} \mathrm{C}$ if it was linear. 


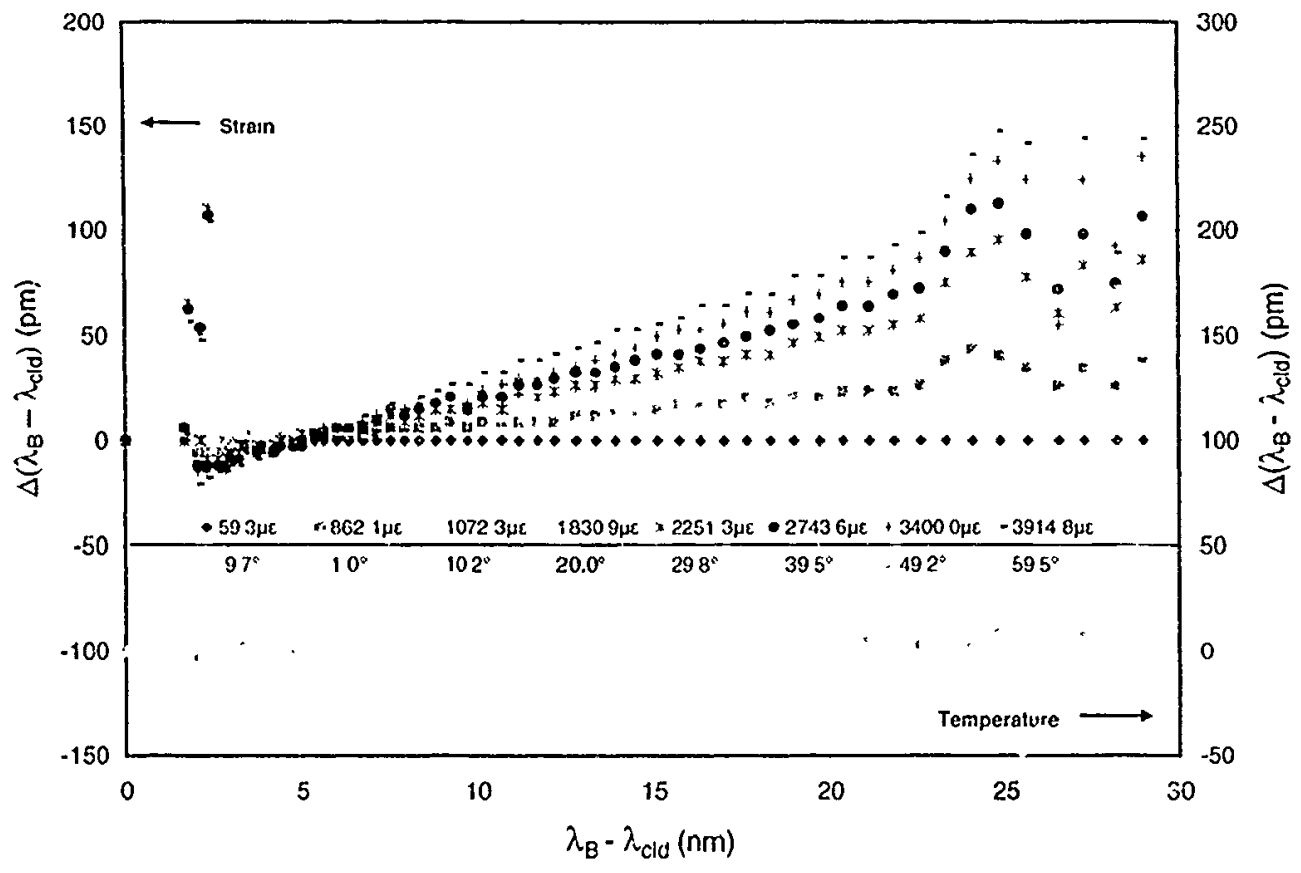

(a)

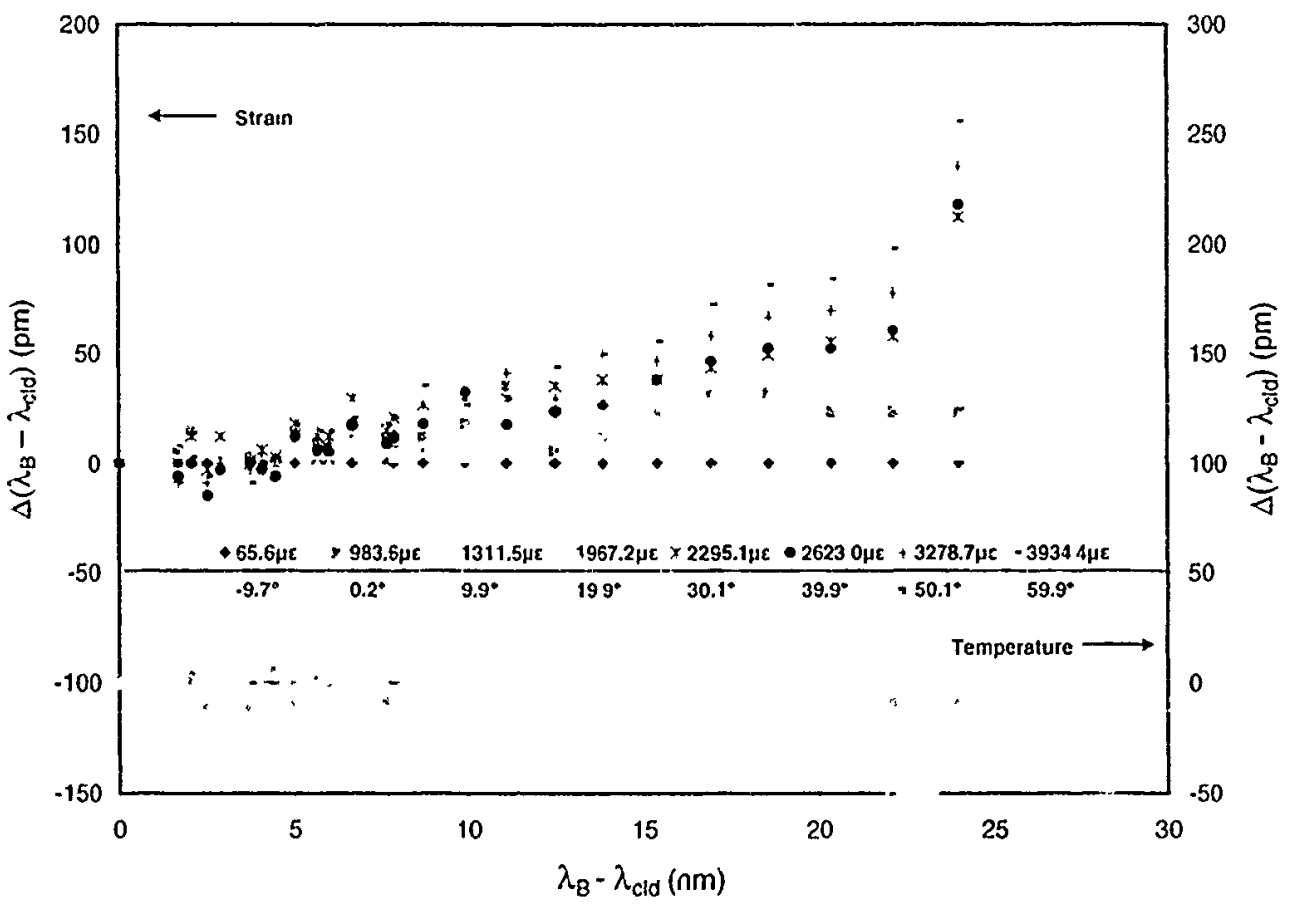

(b)

Figure 5.21: The relative wavelength shift of cladding mode resonances to the Bragg resonance of TFBGs (a) with standard SMF-28 fibre $d=125 \mu \mathrm{m}$ and $\theta=4^{\circ}$ due to longitudinal strain and temperature Perturtat:ions, (b) with thin cladding layer $\mathrm{d}=50 \mathrm{sm}$ and $0=6^{\circ}$ due to longitudinal strain and temperab a enturbations. 
Fig. 5.21(b) shows exactly the same measurements for the experimental fibre with $50 \mu \mathrm{m}$ cladding diameter and $\theta=6^{n}$. While the appearance of the graph is similar to Fig. 5.21(a), there are notable differences. Most importantly, the resonance shift behaviour with increasing strain is much less regular, especially for low strain values and the maximum variations due to temperature changes between $-9.7^{\circ}$ to $59.5^{\circ}$ are about two times larger (between -41 to $+17 \mathrm{pm}$ or $0.8 \mathrm{pm} /{ }^{\circ} \mathrm{C}$ ). It appears as if the linear regular region of "well behaved" modes observed between -5 and $-20 \mathrm{~nm}$ from the Bragg resonance in standard fibre has been squeezed away in the reduced cladding fibre, leaving only complex resonances made up of multiple vector modes [12] in the low mode order region and for modes nearing cut-off.

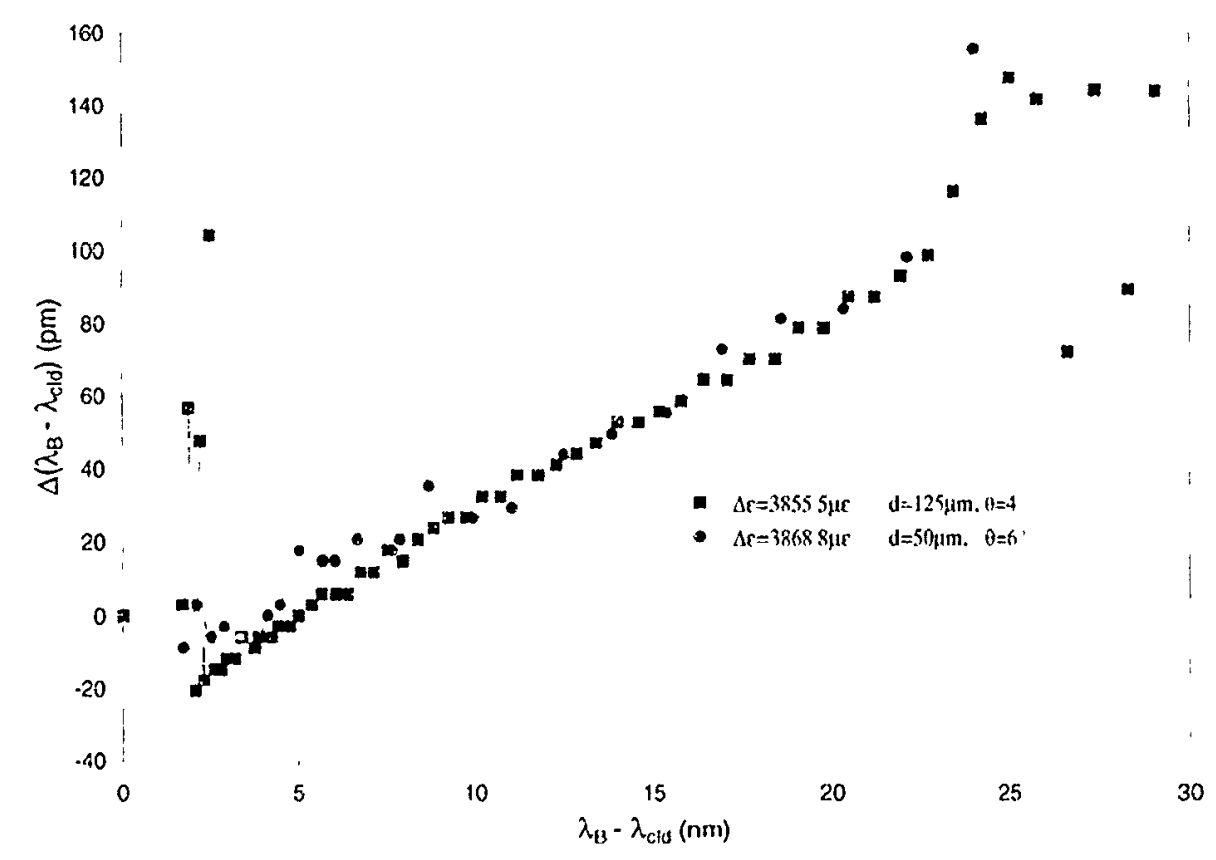

Figure 5.22: Differential wavelength shift of individual resonances for thin and regular fibres near $3860 \mu$ s 
A direct comparison of the two types of fibres for a high strain value in each case (3855 $\mu \mathrm{m}$ and $3868 \mu \mathrm{m}$ ) is provided in Fig. 5.22. Apart from irregular regions at both ends of the mode spectra in each case, the differential strain is equal for the two fibres when considering resonances that are located at the same distance from the Bragg peak. It is clear from this graph that the dominant feature of the differential strain sensitivity is the wavelength spacing between the resonances (or equivalently the difference in the effective index of the cladding mode in question to that of the core mode), even though the mode order (and hence its cross sectional shape across the cladding) is quite different for fibres that are so different in size. It is worth mentioning that the tilt angle was not even the same for these two results, further highlighting the insensitivity of the differential wavelength shift technique to the exact value of tilt angle. Finally, much of the irregular behaviour observed for low and for high order modes could be resolved by a more accurate individual mode resonance position determination for these quasidegenerate resonances made up of several vector modes. 


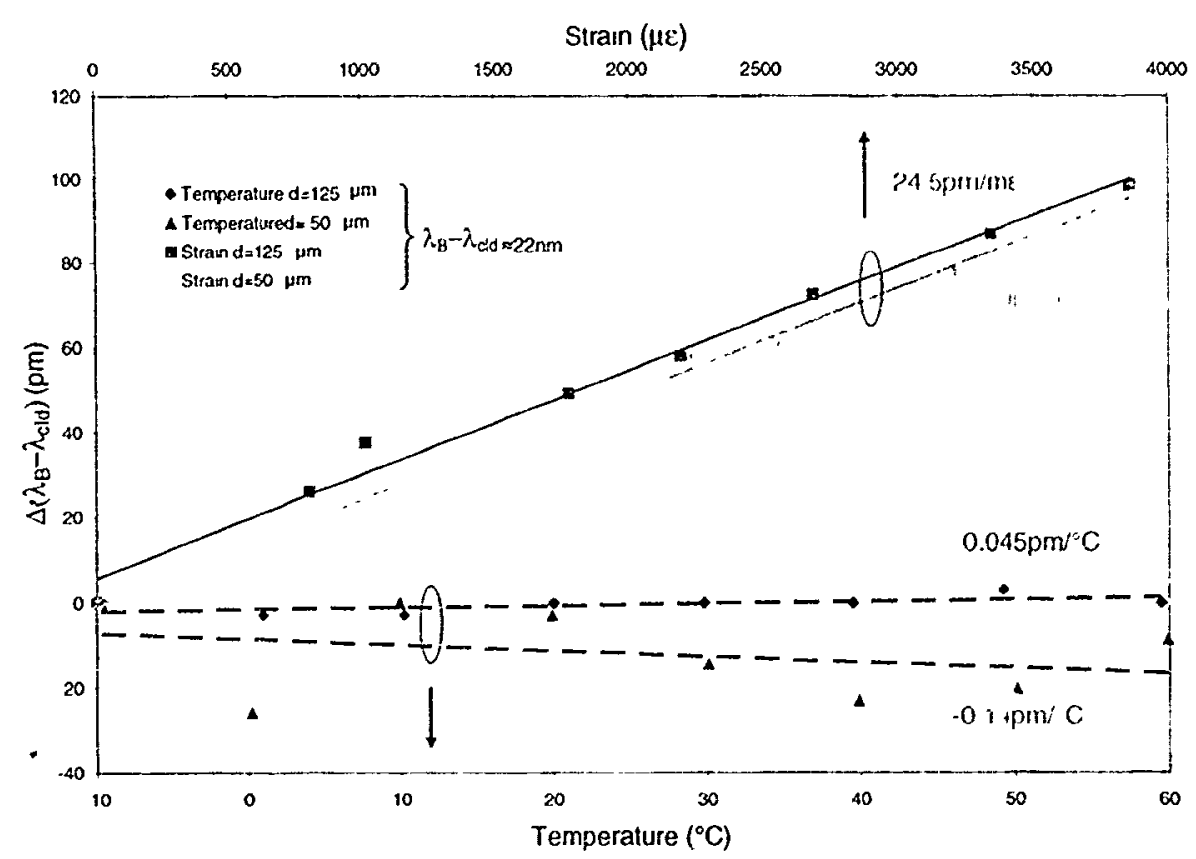

Figure 5.23: Comparison of differential wavelength shifts of cladding mode resonances for a $4^{\circ}$ tilted FBG in SMF-28 fibre and a $6^{\circ}$ tilted FBG in Corning experimental 50 $\mu \mathrm{m}$ diameter thin cladding fibre with $\sim 22 \mathrm{~nm}$ away from their Bragg resonances due to strain and temperature perturbations

Fig. 5.23 shows two single cladding resonance wavelength shift due to strain and temperature perturbations at resonance peaks about $22 \mathrm{~nm}$ away from their Bragg resonances in two different diameter fibres: one is $125 \mu \mathrm{m}$ cladding layer diameter SMF28 fibre and the other is $50 \mu \mathrm{m}$ cladding layer diameter Corning experimental fibre. From the results, we can see that the differential wavelength shifts of two TFBGs increase almost linearly as the strain increases. The strain sensitivity of the differential wavelength shifts are $24.5 \mathrm{pm} / \mathrm{m} \varepsilon$ for TFBG in SMF-28 fibre and $24.9 \mathrm{pm} / \mathrm{m} \varepsilon$ for TFBG in thin cladding layer fibre and the differential wavelength shift. due to the temperature changes are $0.045 \mathrm{pm} /{ }^{\circ} \mathrm{C}$ and $-0.14 \mathrm{pm} /{ }^{\circ} \mathrm{C}$ respectively. If we interrogate these two resonances without independent knowledge of temperature, the error due to temperature fluctuations 
corresponds to an uncertainty of $\pm 129 \mu \varepsilon$ for cladding resonance of TFBG in SMF-28 fibre and $\pm 394 \mu \varepsilon$ for cladding resonance of TFBG in thin cladding layer fibre.

By comparing the results in these two TFBGs, we can see that a thin cladding layer thickness results in fewer cladding modes that are more widely separated in wavelength, therefore it increases the range of external perturbation measurements achievable with a single resonance without facing an uncertainty about which resonance is observed. Unlike grating-based refractive index sensors, in which a thin cladding layer thickness is preferred for high sensitivity of the cladding mode resonance to external refractive changes, a thin cladding layer thickness does not change the strain sensitivity of cladding mode resonances, but it slightly increases the relative temperature dependence of cladding mode wavelength shift to Bragg wavelength shift from $0.35 \mathrm{pm} /{ }^{\circ} \mathrm{C}$ to $0.8 \mathrm{pm} /{ }^{\circ} \mathrm{C}$ (between $125 \mu \mathrm{m}$ and $50 \mu \mathrm{m}$ diameter tibres). We have clearly shown that the strain sensitivity of individual cladding resonances is uniquely determined by the $v$ avelength separation between the resonance and the Bragg resonance, irrespective of cladding diameter. In addition, we showed that achieving thin cladding thicknesses by HF etching of standard fibres reduces the spectial quality of individual transmission resonances especially for high order modes with larger amplitudes at the etched surface (however, for envelope-based interrogation schemes this does not pose a problem [61], and thinned fibres are much easier to splice to conventional fibres).

\subsubsection{Surrounding Refractive Index Sensitivity}

As we know that the thin cladding layer in optical fibre can significantly increase the surrounding refractive index sensitivity for LPG [62] and TFBG [14], here we show our 


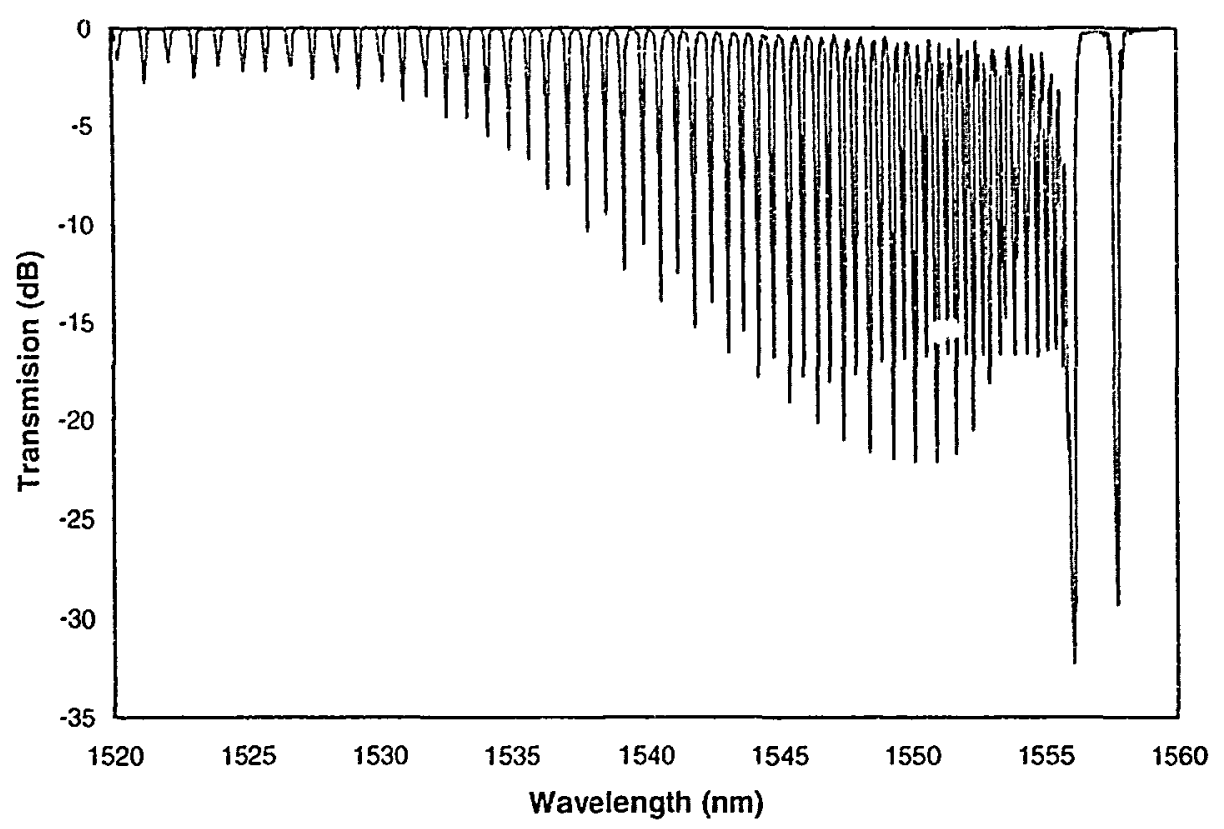

(a)

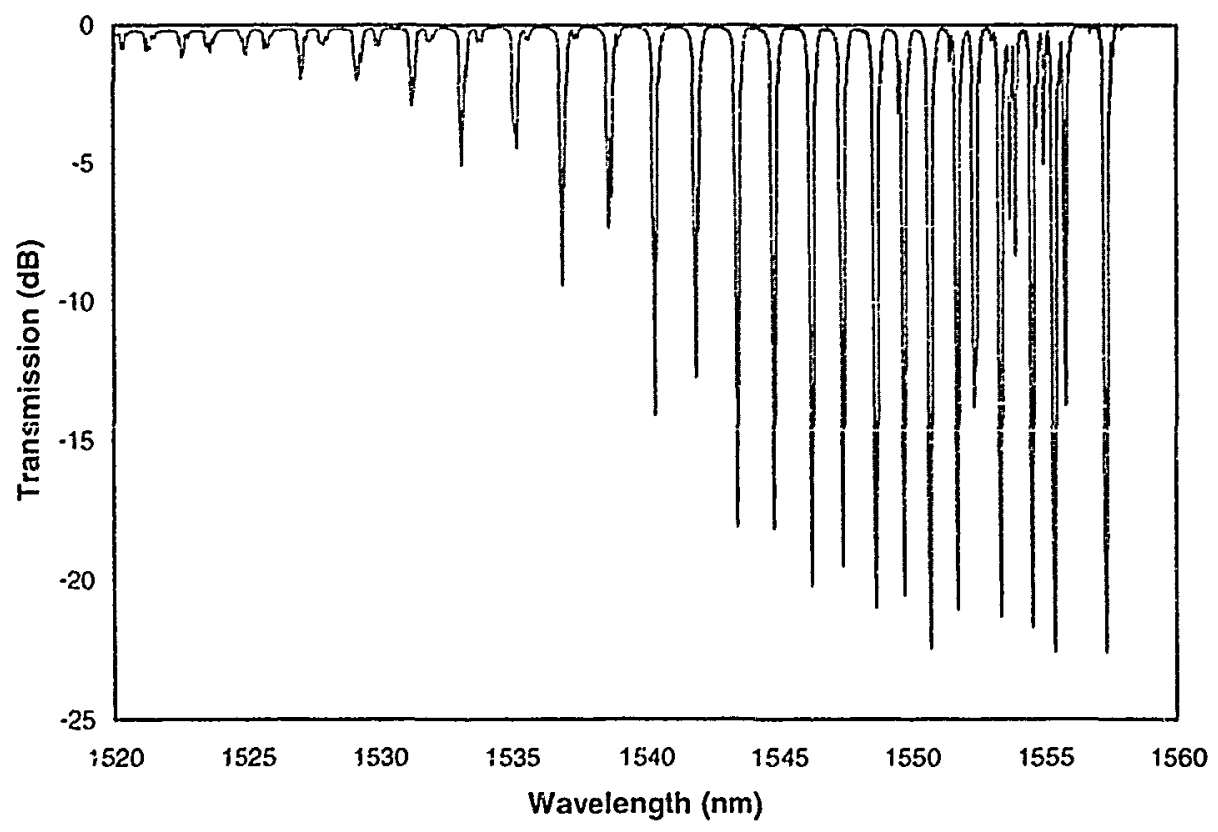

(b)

Figure 5.24: The transmissiorx spectra of weakly TFBG: (a) SMF-28 fibre $d=125 \mu \mathrm{m}$ and $0=4^{\circ}$; (b) Corning experimental thin cladding layer fibre $d=50 \mu \mathrm{m}$ and $0=4^{\circ}$. 
experimental results for TFBG with different fibre cladding layer thickness. The experimental TFBG sensors are all written in hydrogen-loaded CORNING SMF-28 fibres and CORNING thin cladding layer experimental fibre by a $193 \mathrm{~nm}$ ArF excimer laser light and using the same phase mask to generate the grating pattern. Fig. 5.24 shows transmission spectra of two TFBG sensors: (a) the TFBG is written in a SMF-28 fibre with tilt angle $0=4^{\circ}$, (b) the TFBG is written in a CORNING experimental $50 \mu \mathrm{m}$ cladding layer diameter fibre with tilt angle $\theta=4^{\circ}$.

Fig. 5.25 shows experimental results of TFBGs immersed in calibrated refractive index liquid oils from Cargille Corporation: the refractive indices listed $\left(n_{\mathrm{I}}\right)$ are the exact values at a wavelength of $589 \mathrm{~nm}$ (the supplier provides an equation to estimate the values of the refractive indices at the wavelengths we used but this equation has a listed uncertainty of 0.001 ). Fig. 5.25 (a) shows the cladding resonance wavelength shifts due to surrounding refractive index changes of TFBG in SMF-28 fibre. These differential wavelength shifts (relative to the Bragg resonance are equivalent to absolute shifts) since the Bragg resonance is totally immune to SRI. Fig. 5.25 (b) shows the cladding resonance wavelength shifts due to surrounding refractive index changes of TFBG in a thin cladding SMF. It can be seen from Fig. 5.25, when the mode close to cutoff, it has the maximum differential wavelength shift or maximum index sensitivity, because when the mode close to cutoff, the power is less confined in the fibre core and cladding layers, any SRI change can cause the large power perturbations in fibre and surrounding medid. Comparing Fig. 5.25 (b) with Fig. 5.25 (a), we can see cladding mode resonances of TFBG in the thin cladding SMF has much higher inde: wavclength shifts than the 
respective one of TFBG in SMF-28 fibre has the same distance from Bragg resonance. To direc comparing the index sensitivity of those two TFBGs, we chose the cladding mode resonance which is about $22 \mathrm{~nm}$ away from the core mode resonance wavelength, the relative wavelength shifts of cladding mode resonances to the Bragg resonance of TFBG with surrounding refractive index are shown in Fig. 5.26. As we can see those two modes will cutoff when $n_{D}>1.42$, and they have the largest index sensitivity before cut off. For the SRI change from 1.41 to 1.42 , the relative cladding mode resonance wavelength of SMF-28 shifts about $185 \mathrm{pm}$ when the resonance in thin cladding layer SMF shifts $531 \mathrm{pm}$, which is 2.9 time larger than the resonance in SMF-28. So in index sensing applications, the thin cladding layer SMF is preferred than the standard SMF-28 fibres.

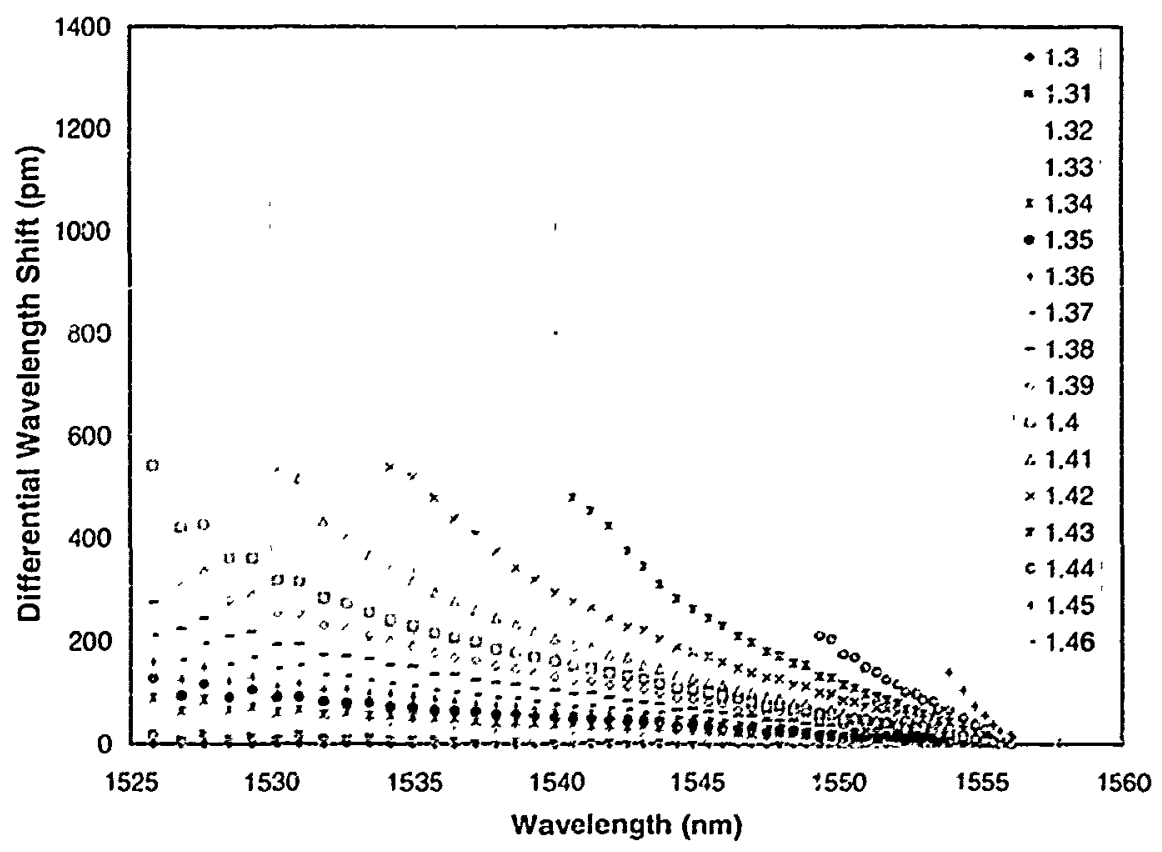

(a) 


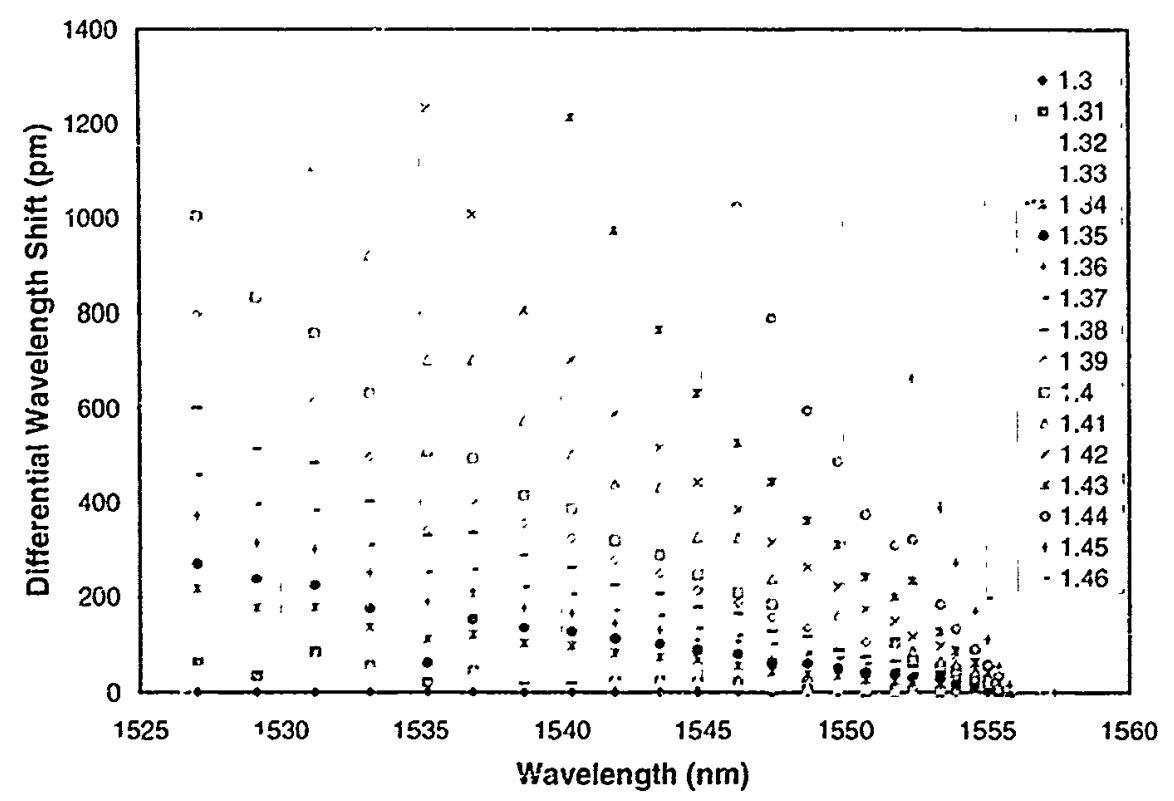

(b)

Figure 5.25: Experimental relative wavelength shifts of cladding mode resonances to the Bragg resonance of FBG with surrounding refractive index $\left(n_{1}\right)$ changes (a) in SMF-28, (b) in $50 \mu \mathrm{m}$ diameter SMF.

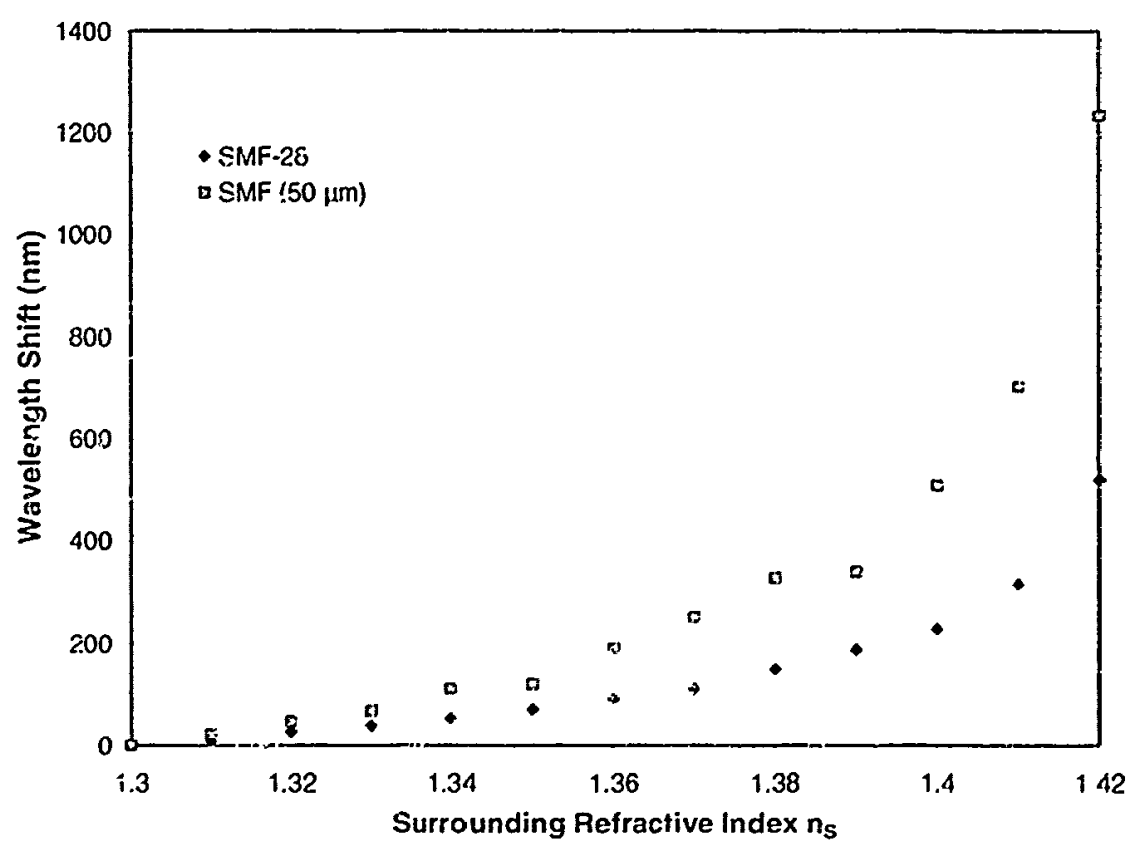

Figure 5.25: The relative wavelength shifts of cladding mode resonances to the Bragg resonance of TFBG with surrounding refractive index $\left(\mathrm{n}_{\mathrm{D}}\right)$ changes (a) in SMF-28, (b) in 50 $\mu \mathrm{m}$ diameter SMF. 


\subsection{Discussion}

In the above, we present analytical and experimental results for sensitivities of TFBGs with temperature, strain and surrounding refractive index perturbations. All the sensitivity we deal with up to now is for tracking the individual core and cladding mode wavelength shifts and the differential wavelength shifts between the core and cladding mode resonances. Other sensor applications of the TFBG are possible such as (i) tracking the core mode and ghost mode reflection powers to perform as a vibration sensor [63]; (ii) tracking the total transmission power with SRI perturbations to perform an index sensor locally by a photodetector [64] or remotely by OTDR [65]; (iii) tracking the radiation mode resonance peak to peak amplitude change in transmission spectrum to perform as an index sensor in which the SRI are higher than fibre cladding and core refractive indices [66] and, (iv) tracking the set of cladding mode resonances, which have the maximum resonance strength in the fibre with a thin gold coating, shifts with SRI changes to perform a very high index sensitivity (comparable with the plasmon mode index sensitivity) index sensor [67]. All those show the big flexibility of TFBG for sensor applications in different areas.

Fig. 5.27 (a) shows schematic diagram of a TFBG with a misaligned fusion for vibration measurement. Because of misalignment in the fibre core, now the ghost mode can re-couple to the core mode and it is shown in Fig, 5.28 (b). The ghost mode is very sensitive to the fibre bending [14], when we glue the TFBG to a metal sheet and push the end of the metal sheet, we can determine the displacement of metal sheet or vibration amplitude and the vibration frequency by measuring the re-coupled ghost mode power 
changes as shown in Fig. 5.27 (c). By tracking the core mode wavelength shift, it is also possible to remove the temperature effect.
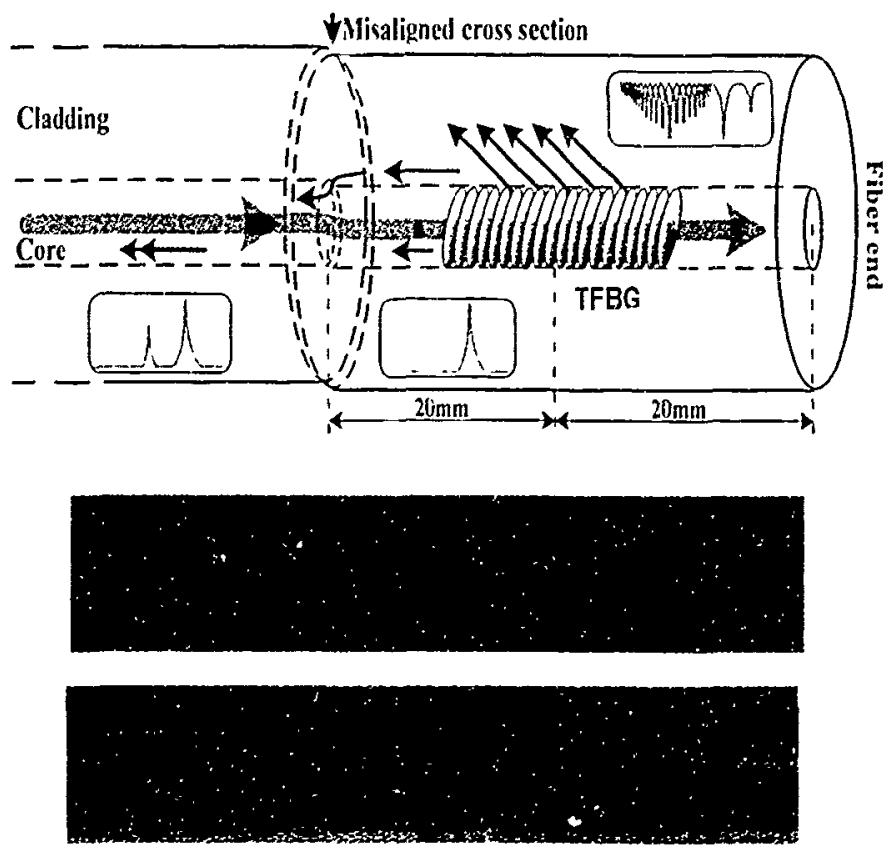

(a)

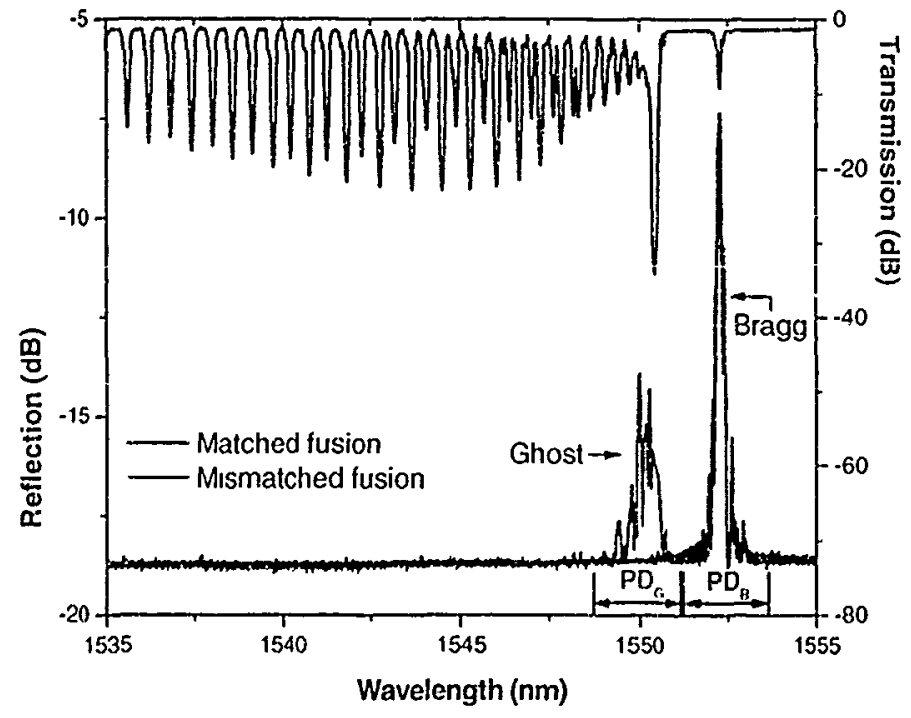

(b) 


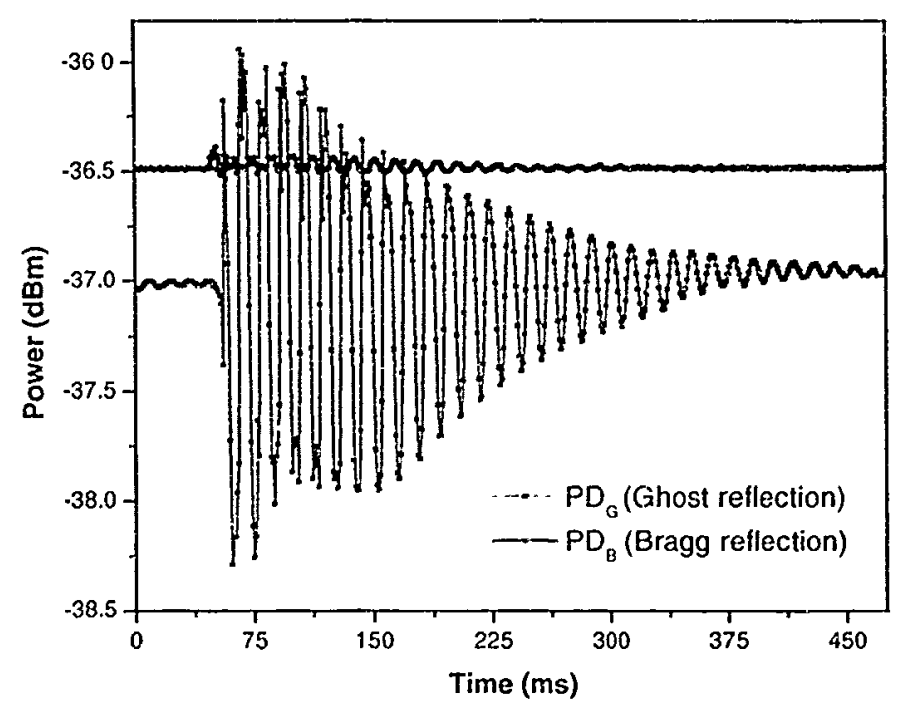

(c)

Figure 5.27: (a) Schematic diagram of a TFBG with a misaligned fusion for vibration measurement and before and after photographs of the fusion splice. (b) TFBG Spectra before and after misaligned junction, (c) Harmonic oscillating response of the sensor system following an impulse excitation [63].

Fig. 5.28 (a) shows the transmission spectra of a $6^{\circ}$ TFBG with different SRIs. It can be seen that as the SRI increases, low order cladding modes are cutoff and become leaky modes. This means less power coupling from the core mode to those leaky modes as the SRI increasing, therefore more transmission power can be detected in transmission as shown in Fig. 5.28 (b). It makes the power detection relatively easy and can make a very low cost SRI sensor. This sensor has high index sensitivity in the SRI close to the fibre cladding refractive index, but relatively low index sensitivity in the SRI from 1.33-1.41. 


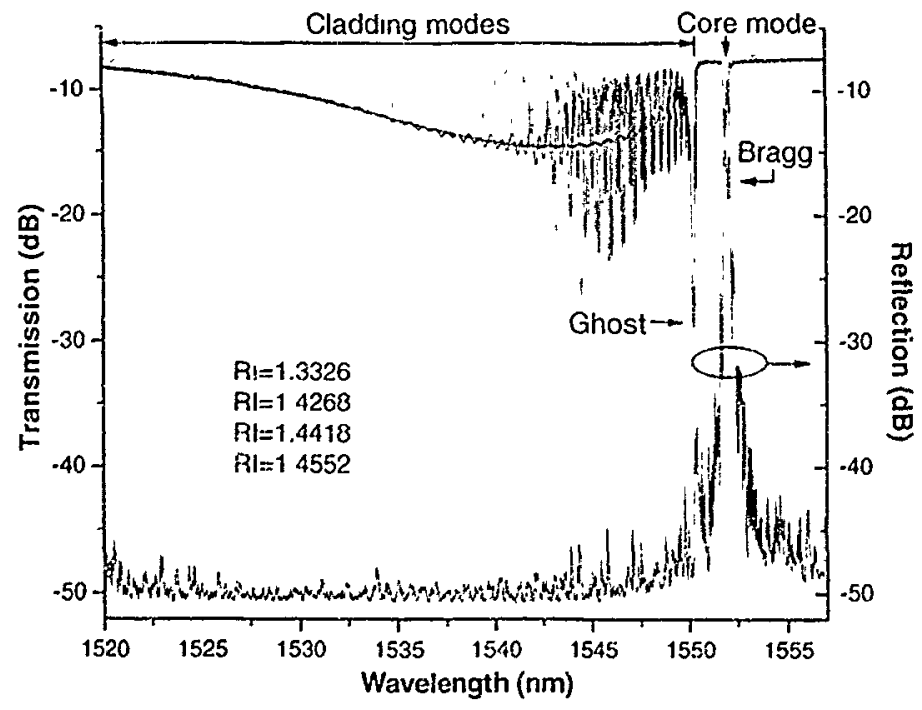

(a)

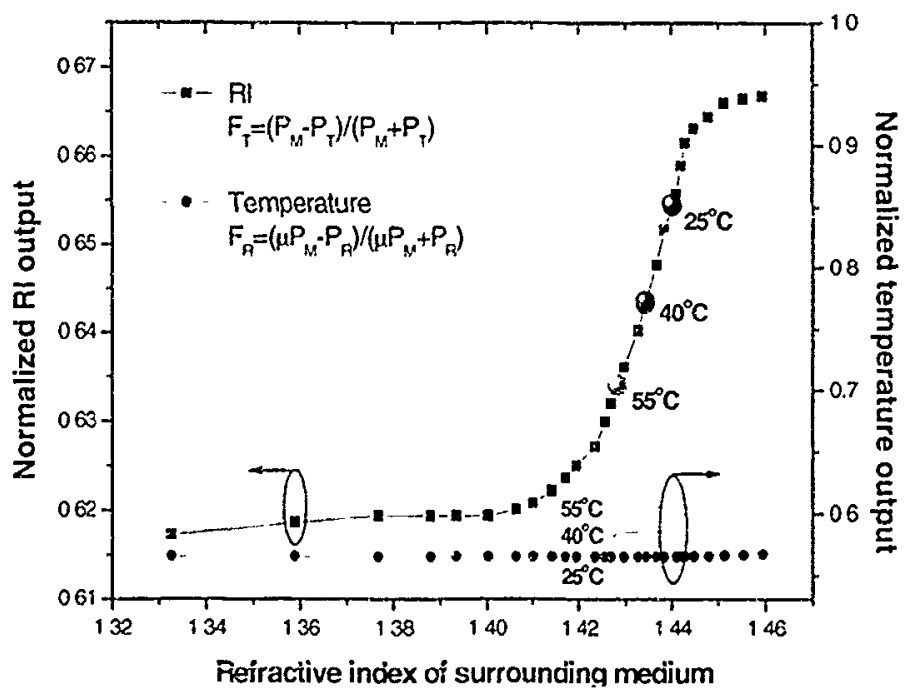

(b)

Figure 5.28: (a) Spectra of $6^{\circ}$ TFBG versus RI at a fixed temperature $\left(25^{\circ} \mathrm{C}\right),(\mathrm{b})$

Normalized power response of $6^{\circ}$ TFBG versus $\mathrm{RI}$ at a fixed temperature $\left(25^{\circ} \mathrm{C}\right.$ : closed symbols), and other temperatures ( 40 and $55^{\circ} \mathrm{C}$ : open symbols) [64].

The TFBG also can be used as an index sensor to sense SRI values higher than the fibre core and cladding refractive indices. Fig. 5.29 (a) shows the transmission spectra of a TFBG immersed in refractive index oil $\mathrm{n}_{1}=1.46-1.54$. Now all cladding modes become radiation modes, and we can see that the radiation mode resonances have a large 
bandwidth comparing with the guided cladding mode resonances. The radiation mode resonance ampitude increases with the SRI increasing as shown in Fig. 5.29 (b). It means I. e can track the radiation mode peak to peak amplitude changes to obtain the SRI value. From Fig. 5.29 (c), we can sie high index sensitivity in the SRI close to the fibre cladding refractive index and low index sensitivity in the SRI from 1.54-1.64. For a power nieasurement resolution of $0.1 \mathrm{~dB}$, the maximum inde $x$ sensitivity could reach $10^{-4}$.

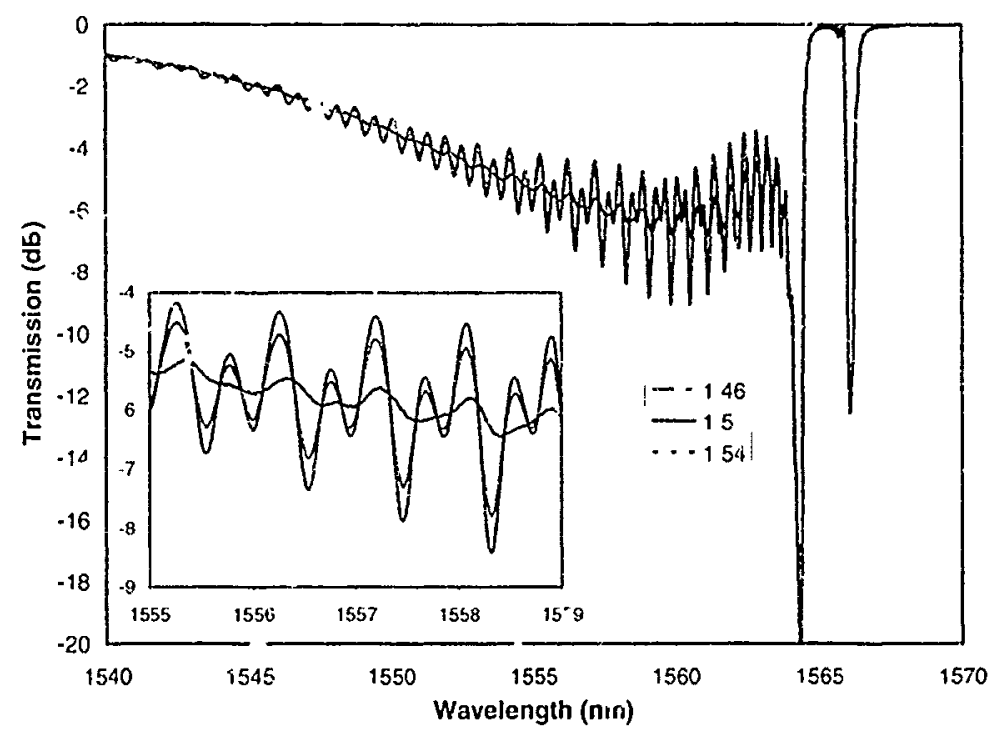

(a)

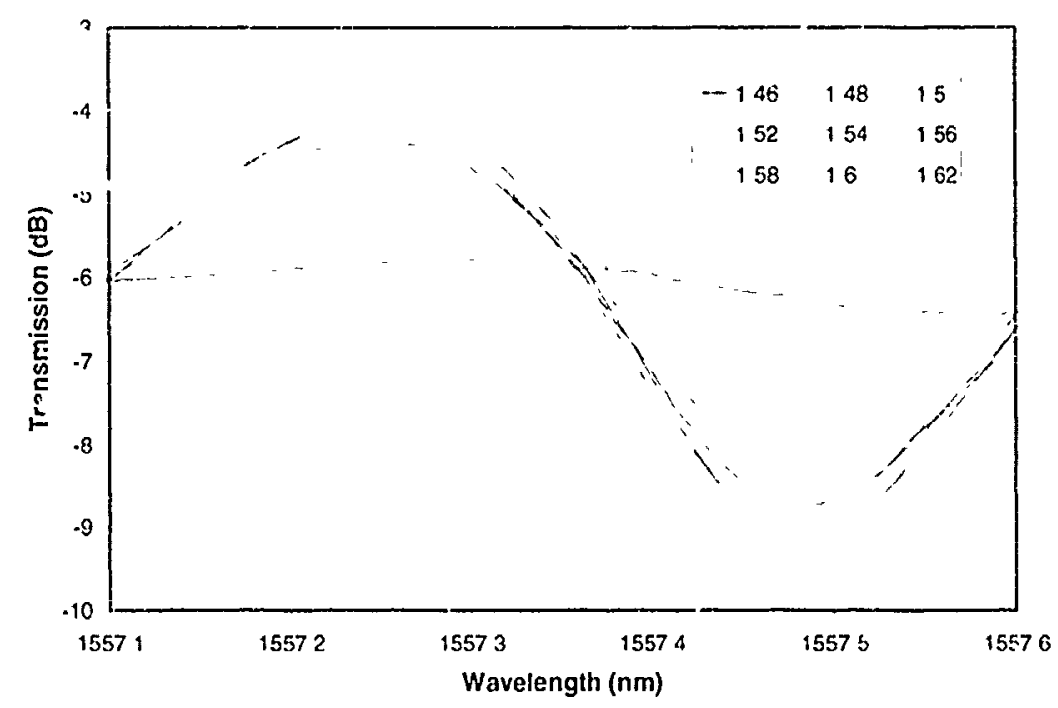

(b) 


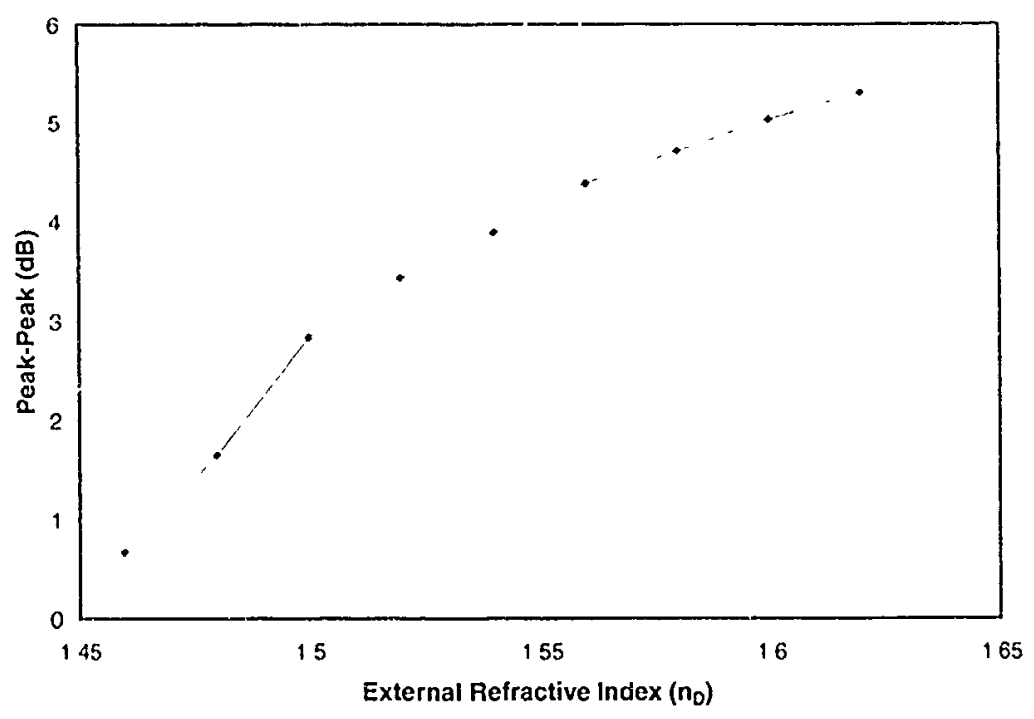

(c)

Figure 5.29: (a) The transmission spectrum of a TFBG with tilt angle $\theta=4^{\circ}$ with SRI higher than fibre cladding refractive index; (b) The transmission spectrum of one selected radiation mode resonance for SRI higher than the fibre cladding refractive index; (c) Peakto-peak value response of the selected radiation mode resonance in different SRI

One big advar. 'Ige for TFBG is that it can excite plasmon modes when the fibre has a metal coating [45]. As we analyzed in the previous Chapter, the resonances between plasmon mode resonances and leaky mode resonances show the strongest resonance peaks as shown in Fig. 5.30 (a) (region labelled $\lambda_{p}$ ). Fig. 5.30 (b) shows the transmission spectrum of the same TFBG without gold coating. Comparing with the Fig. 5.30 (a) and Fig. 5.30 (b), we can see that the fir:t leaky mode resonance is in the same wavelength around $1535 \mathrm{~nm}$, because the cladding mode cutoff is directly related with SRI and the effective index of the cladding mode itself. So the first leaky mode resonance in these two TFBGs will have the same index sensitivity, a d they are equal to the index 
sensitivity of low less cladding mode resonances because those low loss cladding mode resonances are formed by the modes just before cutoff. The low loss cladding mode resonance peak shift is shown in Fig. 5.30 (c). We can directly track the first leaky mode resonance and last cladding mode (just before it is cutoff) in TFBG without gold coating for high index sensing, because it is much more easy to make and reliable for multiple measurement. The recent published paper [67] implied this idea as they have tracked the cladding mode transmission envelope and made a Fourier transform to obtain the response frequency for the index sensing. The difference between the two TFBGs with and without gold coating for biomedical sensor applications could be from the different sensing response time and resolntions, as it is well known that the gold surface bonding effects for DNA, protein and other biomedical materials, therefore the TFBG with the gold coating will take the advantage for high resolution and response time when the TFBG without gold coating for low cost and easy fabrications. It is necessary to further explore the leaky mode characteristics in both TFBGs with or without a thin gold coating to develop new types of biomedical sensors. 


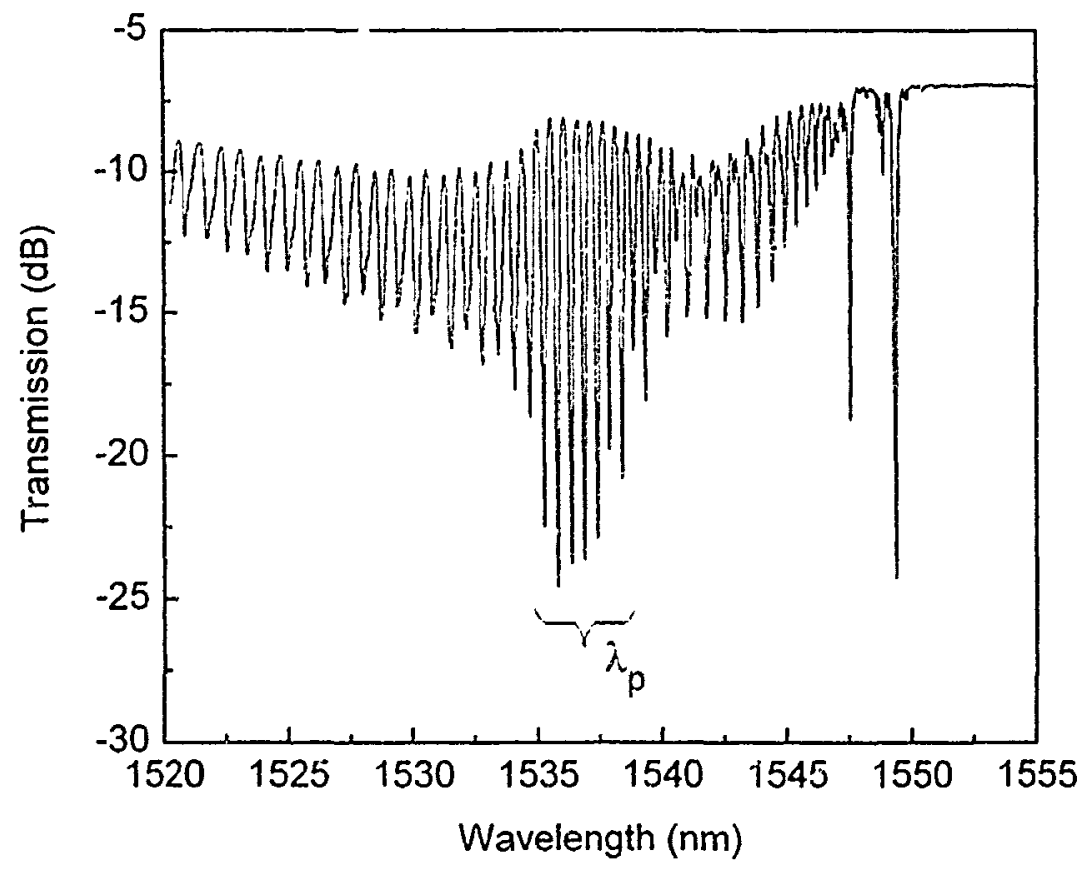

(a)

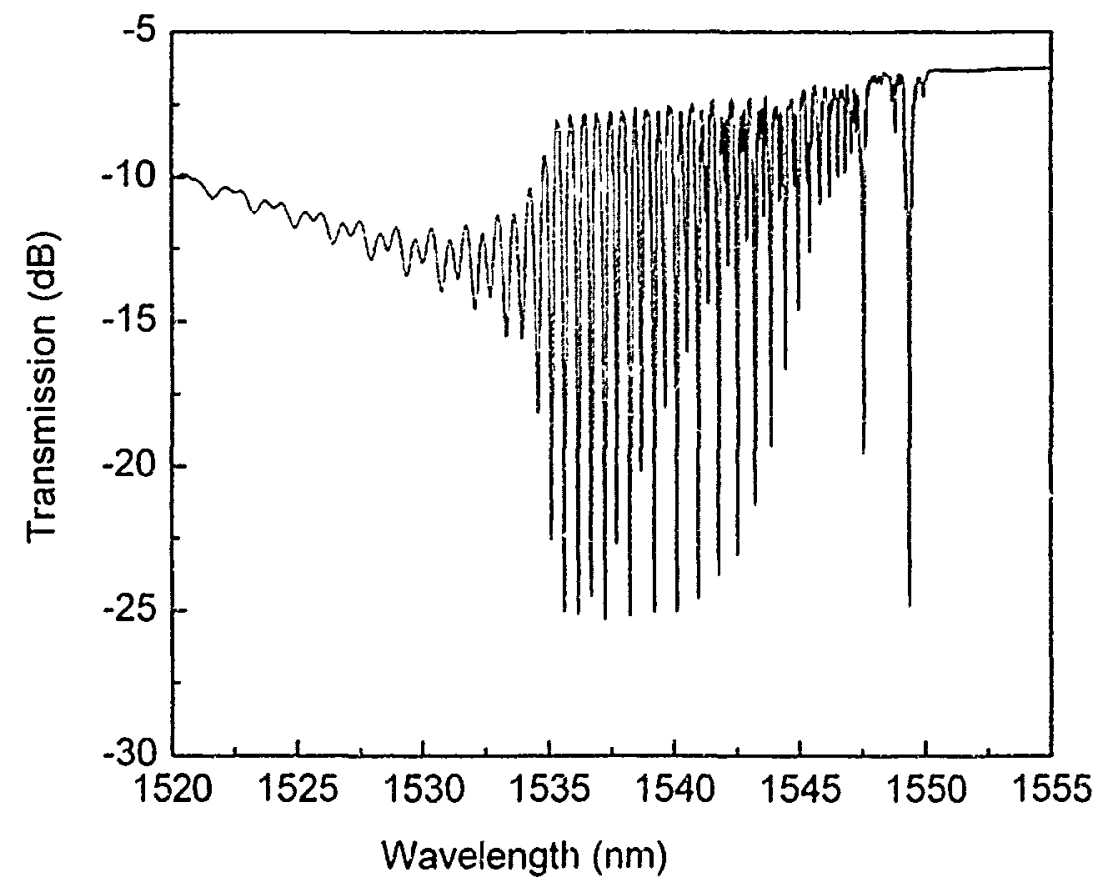

(b) 


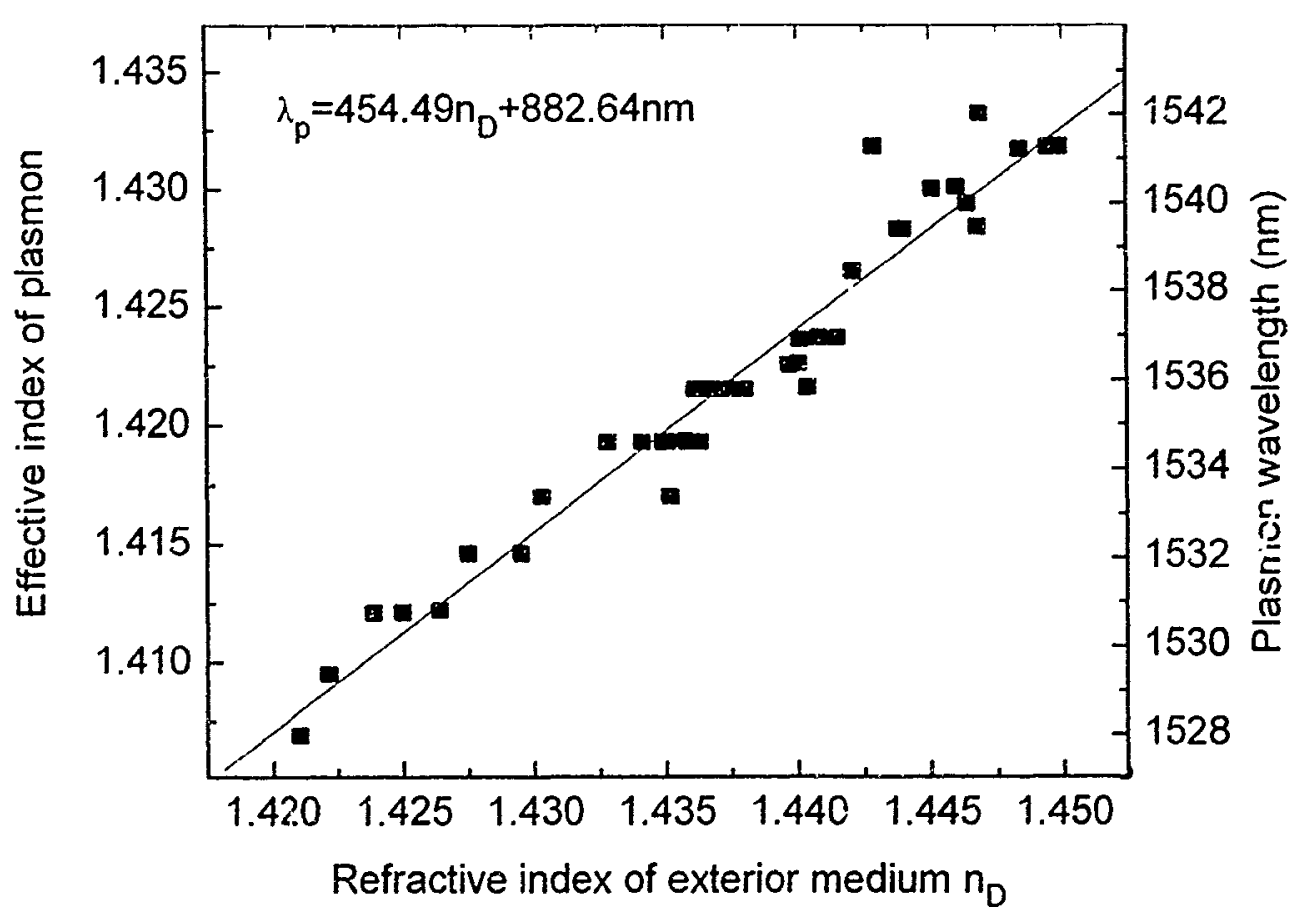

(c)

Figure 5.30: The transmission spectrum of TFBGs in a sucrose solution with $n_{b}=1.4378$ : (a) with a $15 \mathrm{~nm}$ gold coating. The bracket indicates the peak position of the anomalous resonance; (b) without gold coating; (c) dependence of the resonance peak wavelength $\left(\lambda_{\mathrm{P}}\right)$ and corresponding cladding mode effective index on the refractive index of the external medium at $589 \mathrm{~nm}\left(\mathrm{n}_{\mathrm{D}}\right)[45]$.

\subsection{Summary}

In this Chapter, novel sensing mechanisms using TFBGs are analyzed theoretically and confirmed experimentally. The TFBGs can perform temperature-independent strain and refractive index sensing. The TFBG in thin cladding layer SMF will dramatically increase the SRI sensitivity but not provide any advantages for strain and temperature sensing comparing with TFBGs in the standard SMF-28 fibres. By tracking the core mode and cladding mode resonance wavelength shifts, temperature-insensitive strain and 
index sensors can be developed. The TFBG in fibre with a thin gold coating can excite plasmon modes. Those modes are extra sensitive to the SRI changes, but due to the large loss of plasmon modes, their resonance peaks in transmission spectrum is hard to track. However the cladding mode resonances between the plasmon modes and leaky mode resonances show strongest resonances and are very easy to track. The index sensitivity of those low loss modes are comparable with the plasmon modes, combining with the high bonding rate of biomaterial such as DNA and proteins to the gold surface, therefore TFBG with a thin gold coating will be a very good candidate for bio-sensing applications. We have also shown that in addition to the temperature, strain and index sensing applications, the TFBG also can be used for vibration and displacement sensing. 


\section{Chapter 6}

\section{Sensing Characteristics of Core and Cladding Modes in}

\section{Photonic Crystal Fibres}

\subsection{Introduction}

Photonic crystal fibre (PCF) was first demonstrated in 1996 [25] and has been extensively explored for telecommunication and sensor applications [26]. In particular, solid core PCFis have rings of low refractive index inclusions, as air holes for example, along the radial direction, forming an equivalent inner cladding layer which has lower refractive index than the core and the pure silica outer cladding. In such structures, in addition to ą endlessly single modę core mode and conventional cladding modes, there are cladding modes whose fields are confined only within the core and air-hole region $[27,28]$. Those unique characieristics of PCF cladding modes make them very attractive for sensor applications if the modes can be excited and measured in a controlled fashion. While all the modes will be sensitive to the temperature and strain perturbations, only some of the cladding modes will be sensitive to the surrounding media changes. We use photo-written fibre Bragg gratings (FBG) to selectively excite cladding modes in special PCFs that have a small fraction of the core co-doped with germanium $[28,29]$ to increase 
their photosensitivity relative to undoped PCFs [30] and fluorine to keep the refractive index of the doped region close to the index of silica and prevent guidance in that layer.

In the work presented here, strong FBGs are w ritten using a conventional phase mask technique and $193 \mathrm{~nm}$ ArF excimer laser irradiation. Even though the FBG fringes are not tilted with respect to the fibre axis, several strong cladding mode resonances are observed (in the transmission spectrum only) in addition to a $99.9 \%$ reflectivity core mode reflection. The strain, temperature and Surrounding Refractive Index (SRI) sensitivities of all these mode resonances will be presented here and compared with those of the core and cladding modes from tilted FBGs written in standard telecommunication fibres (Corning SMF-28 fibre) with the same laser irradiation set-up [52,53]. In standard fibres, the grating fringes must be tilted relative to the fibre axis in order to excite strong cladding mode resonances. Our experimental results for the relative sensitivity of the various modes will be supported by theoretical considerations and by simulations of the mode profiles of our fibre. Finally, we will discuss potential multiparameter optical fibre sensing applications based on the observed differential sensitivities of the resonances of these gratings. Of particular interest is the excitation of narrowband cladding mode resonances wit.i the low temperature sensitivity of short period Bragg gratings and strong differential sensitivities to SRI and strain. While it is outside the scope of the present paper to compare this potential application to the wide variety of competing approaches for multiparameter sensing with fibre gratings [68], our PCF-FBGs present a certain number of unique features that may prove uscful in certain applications. 


\subsection{Experimental Conditions}

The cross section of the experimental PCF fibres used in our work is shown in Fig. 6.1. The period between the air holes is $3.85 \mu \mathrm{m}$ and the air hole diameter is approximately $1.75 \mu \mathrm{m}$. The core is roughly $6 \mu \mathrm{m}$ in diameter with a $1.9 \mu \mathrm{m}$ diameter photosensitive region ( $3 \mathrm{wt} \%$ germanium and $0.9 \mathrm{w} t \%$ fluorine to reduce the index). The net index increase in the doped region is roughly $6 \times 10^{-4}$ above the background pure silica which is about 8 times less than the index step of conventional telecommunication fibre. We used fluorine instead of boron to decrease the index of the germanium-doped region because it would have required too much boron to achieve the same reduction and resulted in higher losses. Note that the diameter and refractive index of the doped region of the core were designed to be small enough to prevent light from being guided by this region (as confirmed by the results below). Guidance is thus totally caused by the holcy cladding that contains 108 air holes. The PCF fibres are spliced with short pieces of SMF-28 to plug both ends before being hydrogen loaded at a pressure of 170 atm for 12 days and roomotemperature to enhance the photosensitivity. The SMF-28 fibres on both ends of the ${ }^{n} \mathrm{CF}$ fibre prevent the rapid out-diffusion of the hydrogen from the fibre due to the airholes. 

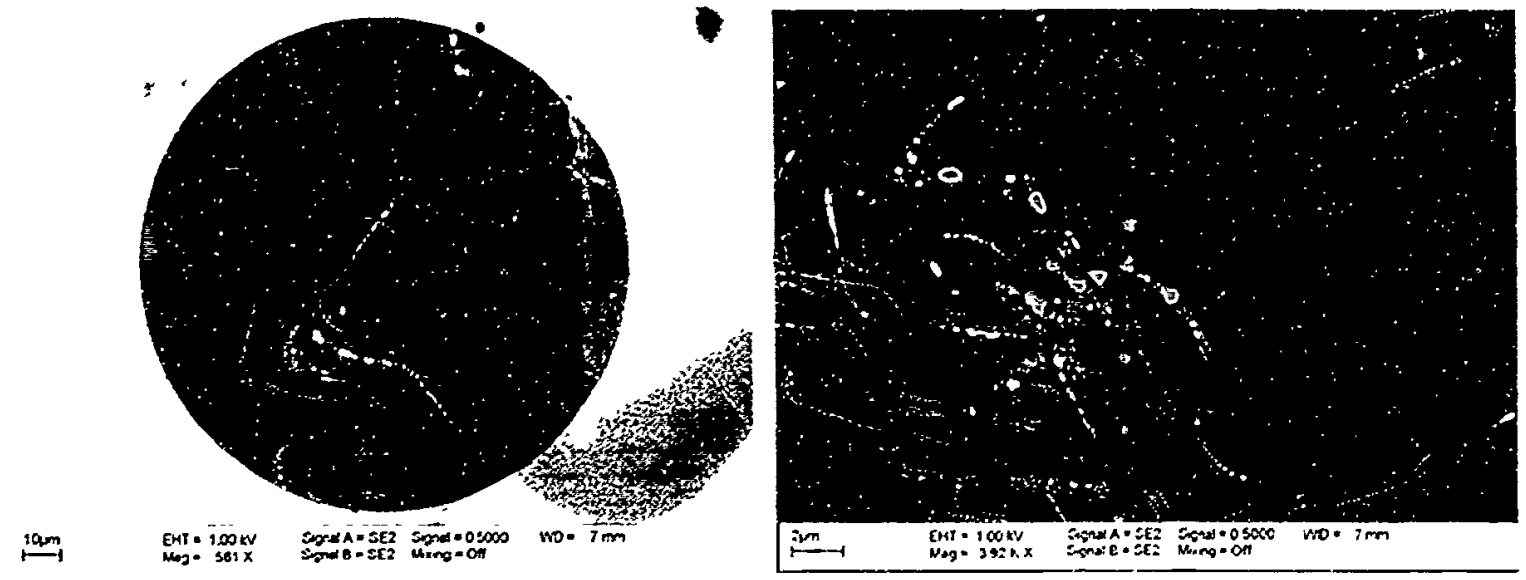

Figure 6.1: The cross section of the photonic crystal fibre used in the experiment (outside fibre diameter $=125 \mu \mathrm{m})$. The doped core is not visible in these photggraphs.

The set-up for writing FBGs in PCF fibres is the same as for SMF-28 fibre, except that when writing in PCF fibres, during the alignment of the fibre at a low laser repetition rate $\left(\mathrm{few}^{\mathrm{H}} \mathrm{Hz}\right.$ ), the fibre is rotated around its axis until a maximum amount of light is transmitted acros: the air hole pattern to reach the fibre core [68]. Once this alignment is achieved, the fibre is fixed in place, about 50 um from the phase mask and the writing as such is carried out (typica!ly for 1-2 minutes at $100 \mathrm{~Hz}$ ). We use $193 \mathrm{~nm}$-wavelength ArF excimer laser light, with a fluence per pulse of approximately $100 \mathrm{~mJ} / \mathrm{cm}^{2}$. The phase mask has a period of $1106.5 \mathrm{~nm}$ and the length of the gratings written in the PCF fibre is $1 \mathrm{~cm}$. The FBG fabrication method used is completely standard (apart from the fibre rotation mentioned above), and yields usable gratings with great reliability. Furthermore, since we do not rely on absolute wavelength or power measurements in the proposed sensor implementations mentioned below (only relative values), minor variants in grating strength or wavelength have no impact on sensor performance. The measurement error 
for the individual resonance peaks is $\pm 3 \mathrm{pm}$, the resolution of the IDSU SWS-OMNI-2 swept wavelength system that are used for measuring transmission spectra. For wavelength differences, the error becomes $\pm 6 \mathrm{pm}$.

\subsection{Core and Cladding Mlode Resonances in PCF Fibres}

\subsubsection{The Spectrum Characteristics of FBG in PCF Fibre}

Fig. 6.2 shows the measured FBG transmission spectrum of a typical grating in our PCF fibre: in addition to the strong fundamental core mode (Bragg) resonance (that also appears in the reflection specirum), there are several other narrowband non-reffective resonances with relatively large amplitude. These extra resonances can be attributed to a coupling of the forward propagating core mode to backward propagating cladding modes that do not propagate back all the way back to the measurement set-up.

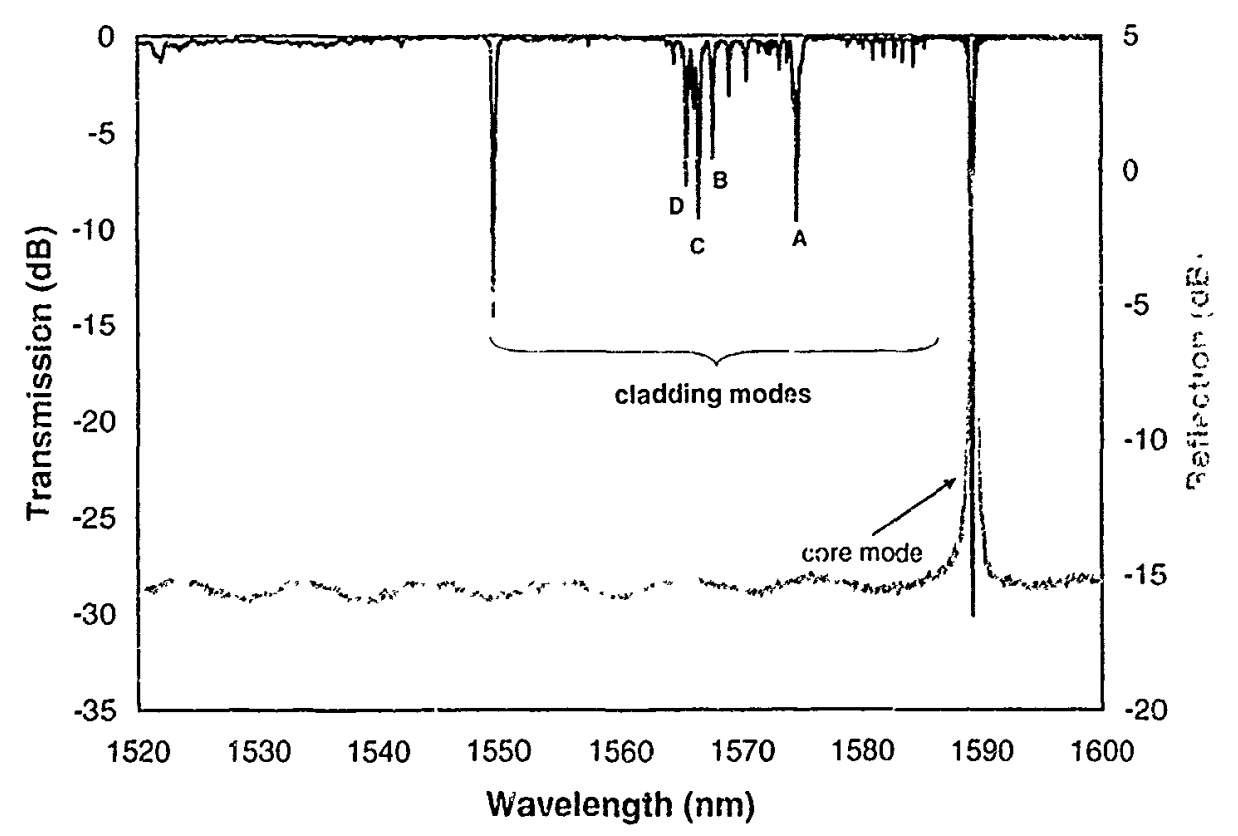

Figure 6.2: The transmission and reflection spectra of a $1 \mathrm{~cm}$-long FBG in PCF fibre. 
Table 6.1 gives quantitative details about those modes that have attenuations larger than $5 \mathrm{~dB}$ : their wavelength, amplitude, and relative distance from the Bragg resonance $\left(\lambda_{\mathrm{B}}\right.$ $\lambda_{\text {cladding }}$. The effective indices are obtained from the following standard formulas [53]: $n_{e f f \text {.core }}=\lambda_{B} / 2 \Lambda$ and $n_{e f f, \text { cludtldng }}^{i}=\lambda_{\text {cluldung }}^{i} / \Lambda-n_{\text {eff.cure }}^{t}$, where $\Lambda$ is half the mask period. Taking into account the precision of our measurement system $(3 \mathrm{pm})$, the line width of the resonances $(100 \mathrm{pm})$ and the uncertainty associated with the dispersion of the core effective index at wavelengths where the cladding resonances occur, we estimate that the effective indices of Table 6.1 are accuratc to one part in $10^{4}$.

The Bragg resonance transmission is lower than $-30 \mathrm{~dB}$ (reflectivity>99.9\%) and its effective index (1.4363) is lower than that of silica at that wavelength (1.4436) thereby confirming that the core mode is not guided by the small doped section of the core. The strong cladding mode resonances seen in Fig. 6.2 are normally not happening in a well written FBG in conventional fibre unless a tilt angle is added [52]. We verified that the fringes generated by our writing set-up were not tilted by writing control gratings in SMF-28 fibres. Such strong cladding mode resonances are mainly caused by the fact that these cladding modes have strong field overlap in the core region [28] and that the grating is formed in a rather small portion of the core (the $1.9 \mu \mathrm{m}$ diameter doped center), thereby enhancing the core to cladding modes couplings [69]. For practical purposes, only those modes which resonance amplitudes larger than $5 \mathrm{~dB}$ are investigated in the following for strain, temperature and surrounding refractive index (SRI) sensitivity. 
Table 6.1: Summary of transmission resonance properties.

\begin{tabular}{ccccccc}
\hline Mode & $\begin{array}{c}\text { Resonance amplitude } \\
(\mathrm{dB})\end{array}$ & $\begin{array}{c}\lambda \\
(\mathrm{nm})\end{array}$ & $\begin{array}{c}\mathrm{n}_{\mathbf{e}^{\prime}} \\
(\exp )\end{array}$ & $\begin{array}{c}\mathrm{n}_{\text {eff }} \\
(\mathrm{sim})\end{array}$ & $\begin{array}{c}\Gamma \\
(\mathrm{sim})\end{array}$ & $\begin{array}{c}\lambda_{\mathrm{B}}-\lambda_{\text {clad }} \\
(\mathrm{nm})\end{array}$ \\
\hline Core & -30.13 & 1589.207 & 1.4363 & 1.4358 & 0.104 & \\
A & -9.67 & 1574.583 & 1.4096 & 1.4091 & 0.014 & 14.624 \\
B & -6.37 & 1567.611 & 1.3969 & 1.3963 & 0.003 & 21.596 \\
C & -9.49 & 1566.475 & 1.3949 & 1.3941 & 0.011 & 22.732 \\
D & -7.78 & 1565.471 & 1.3931 & 1.3927 & 0.005 & 23.736 \\
I & -13.23 & 1549.675 & 1.3643 & 1.3635 & 0.054 & 39.532 \\
\hline
\end{tabular}

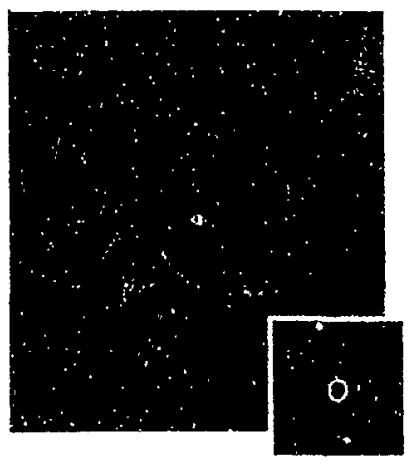

(a) Core Mode

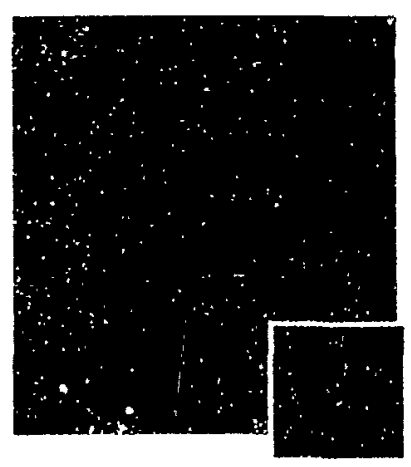

(d) Mode C

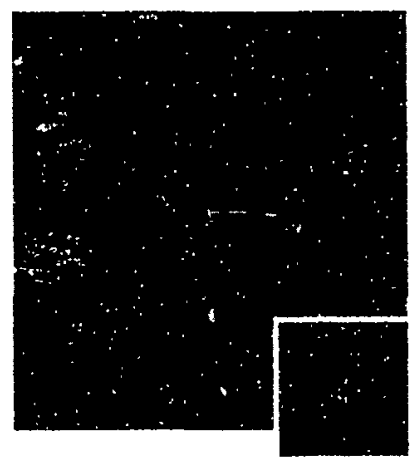

(b) Mode A

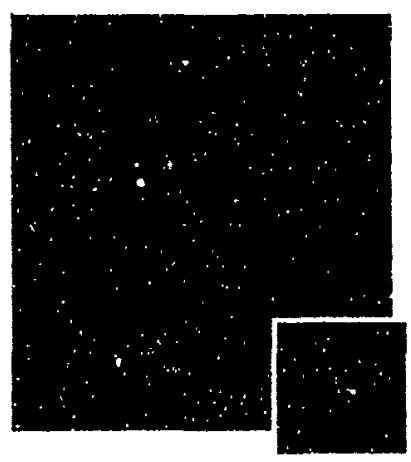

(e) Mode D

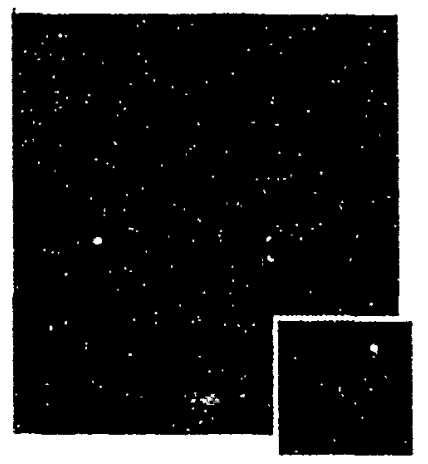

(c) Mode B

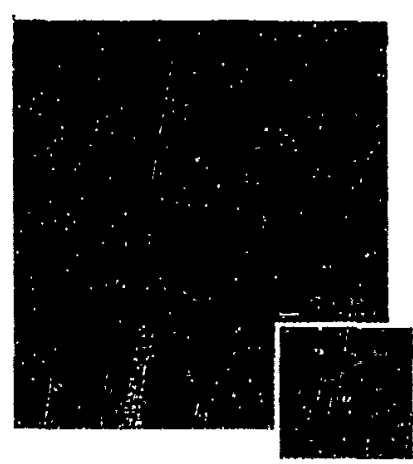

(f) Mode I

Figure 6.3: The electric field intensities of the core mode and cladding modes, the insets show an enlarged view of the core area. 


\subsubsection{Simulations of the PCF Modes}

Before reporting on further experimental results, and in order to better understand the various modal sensitivities reported here, the modes of our PCF fibre have been analyzed by using a mode solver based on the finite element method: Lumerical MODE Solutions from Lumerical Solutions Inc. The simulation window is $140 \mu \mathrm{m}$ by $140 \mu \mathrm{m}$ and perfectly matched layers are used as borndary conditions. The best match between the experimental effective index values and the simulation were obtained with a refractive index of 1.4514 in the doped region (the refractive index of the remainder of the glass is calculated from the Sellmeier equations for fused silica and is equal to 1.44383). The index of the doped region is significantly higher here $\left(7.6 \times 10^{-3}\right)$ than in the as-drawn fibre $\left(6 \times 10^{-4}\right)$ because $o_{1}$ the relatively large index incredse resulting from irradiating the hydrogen loaded doped silica. The refractive indices are 1.4514 in the doped region and 1.44383 for silica elsewhere. The simulated effective indices are given in Table 1 and the corresponding mode field distributions are shown in Fig. 6.3. It can be seen that fields of modes A to D extend all the way to the outer boundary while mode I is confined by the air hole structure that surrounds the core region. For each mode, the inset shows the detail of the mode structure in the vicinity of the core: all of these modes have strong field amplitude in the center of the fibre, where the doped region and hence the grating modulations are located. Not all mode field distributions shown the six-fold symmetry, which could be caused by the mesh size in simulations. The all field distributions show the two-fold symmetry, and six-fold symmetry also obeys the two-fold symmetry. The confinement factor of each mode in the doped section ( $\Gamma$ in the Table 6.1), explains the 
relatively strong coupling between the core and cladding modes in the absence of tilt in the grating planes. For comparison, the confinement factors for the core mode and a typical cladding mode in standard fibre are 0.8 and 0.03 respectively, leading to poor overlap and weak coupling when no grating tilt is presented.

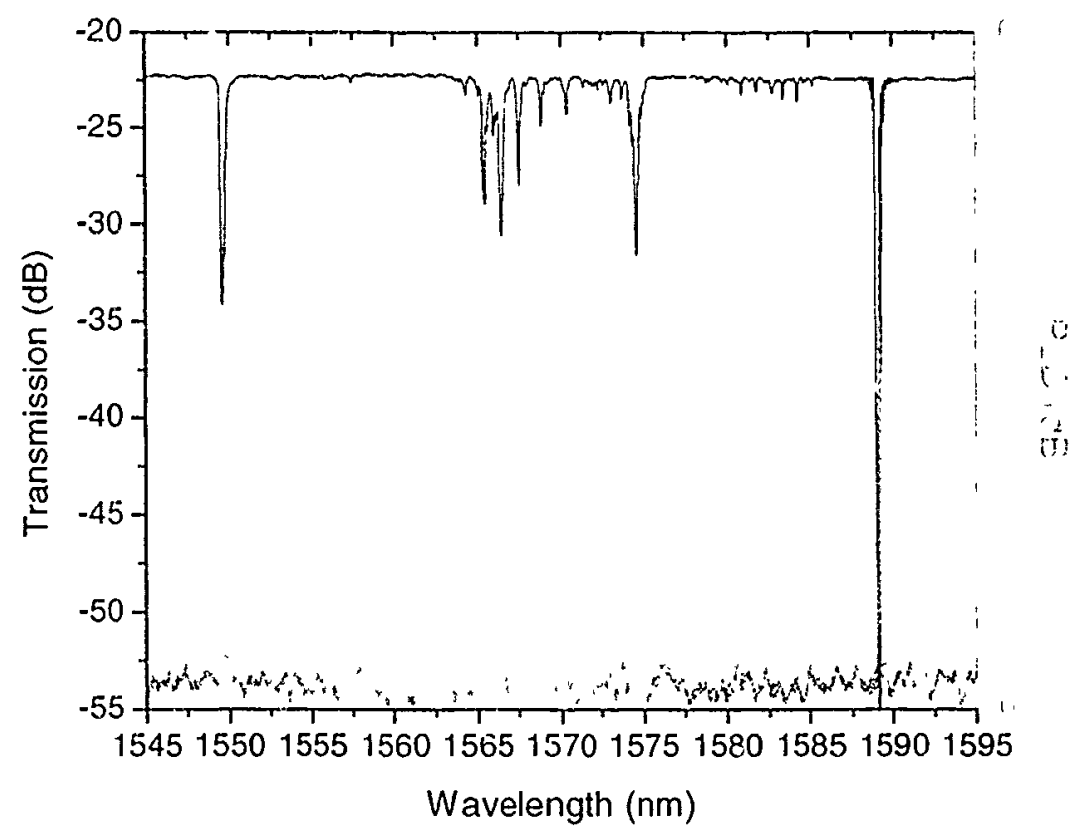

Figure 6.4: The polarization dependent loss of core and cladding modes.

\subsubsection{Polarization Dependence of the PCF Mode Resonances}

The six-fold symmetrical characteristics of PCFs make them inevitably strongly polarization dependent. The doped core exposure to the excimer laser light when FBGs are written also increases the nonuniformity in the cross-section of the core area. Fig. 6.4 shows the polarization dependent loss (PDL) of the core and cladding mode resonances. It can be seen from Fig. 6.4 that the PDL of each mods is dependent on the resonance peak amplitude, for example the core mode has the largest resonance amplitude and the largest PDL. Fig. 6.5 further demonstrates the core and cladding modes polarization 
dependence characteristics through at measure of the transmission spectra with different polarization states. The states are $\mathrm{S} 1$ : circular polarization, $\mathrm{S} 2: 0^{\circ}$ linear polarization, $\mathrm{S} 3$ : $90^{\wedge}$ linear polarization and the S4: $-45^{\wedge}$ linear polarization. From the insets of Fig. 6.5 , the cladding mode resonance shape and amplitude both change with different polarication states of the laser source. Because each cladding mode resonance is formed by a set of individual cladding modes (similar to the HE/EH or LP modes in SMF), each with certain vectorial states, some cladding mode resonances appear to change with polarization because coupling constants of the individual vectorial modes involved depened on the polarization. Due to the six fold air-hole symmetrical structure of the PCF, the core and cladding mode resonances are strongly polarization dependent, the polarization states used for Fig. 6.5 might not be the best choice to maximize the differences between the polarization states. Further polarization dependence weasurements are needed to further explore the characteristics of the FBGs in PCFs.

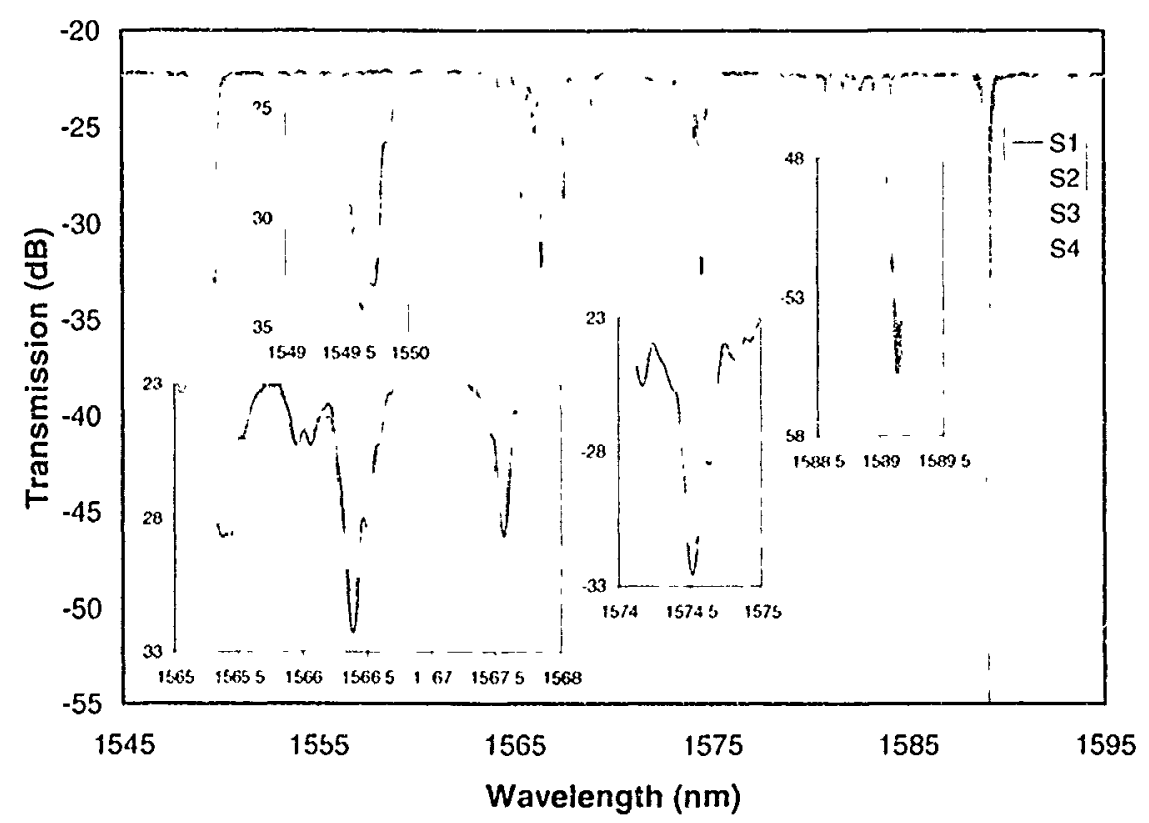

Figure 6.5: The Transmission Spectra of FBGs in PCF with Different Polarization States 


\subsection{The Sensitivity of the Core and Cladding Modes of PCFs}

\subsubsection{Strain and Temperature Sensitivity}

The characterization of the strain sensitivity of individual resonances was carried out by stretching a grating rigilly bonded to two micrometric translation stages. The Fig. 6.6 shows the core mode resonance shift with the axial strain perturbations and Fig. 6.7 shows the cure mode strain sensitivity. Table 6.2 summarizes the core and cladding mode strain sensitivity in these measurements, indicating small differences between the modes. These small differences can be monitored very precisely by using the Bragg mode resonance as a power and wavelength reference [52,53] and Fig.6.8 shows the result of such ralative measurements. The differential strain sensitivity of the different modes is seen to increase with the distance of the resonance from the Bragg peak, a behaviour that was observed previously for the cladding mode resonances of TFBGs in conventional fibres (Corning SMF-28) [70].

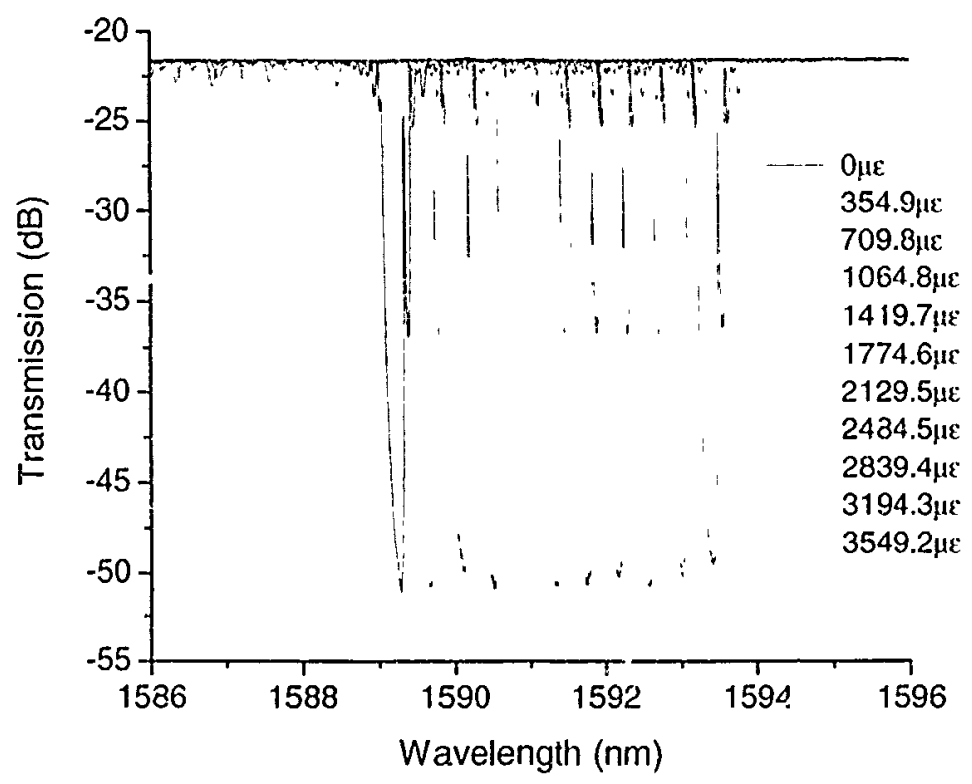

Figure 6.6: The core mode resonance shift under axial strain perturbations 


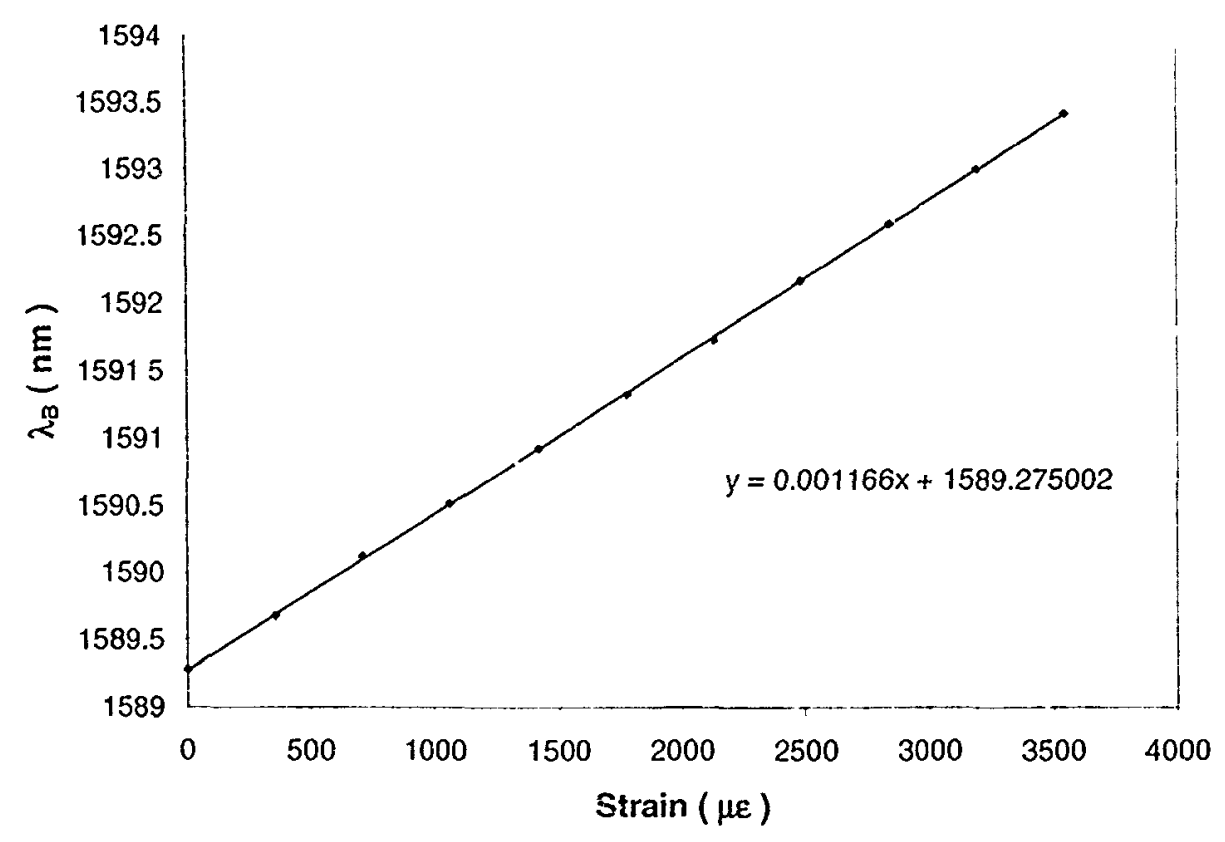

Figure 6.7: The strain sensitivity of the core mode

Table 6.2: Core and Cladding Mode Temperature and Strain Sensitivities

\begin{tabular}{llllllll}
\hline Mode & Core & A & B & C & D & I \\
\hline $\mathrm{d} \lambda / \mathrm{dT}(\mathrm{pm} / \mathrm{C})$ & 10.46 & 10.55 & 10.61 & 10.68 & 10.68 & 10.69 \\
$\mathrm{~d} \lambda / \mathrm{d} \varepsilon(\mathrm{pm} / \mu \varepsilon)$ & 1.166 & 1.150 & 1.141 & 1.140 & 1.137 & 1.119 \\
\hline
\end{tabular}




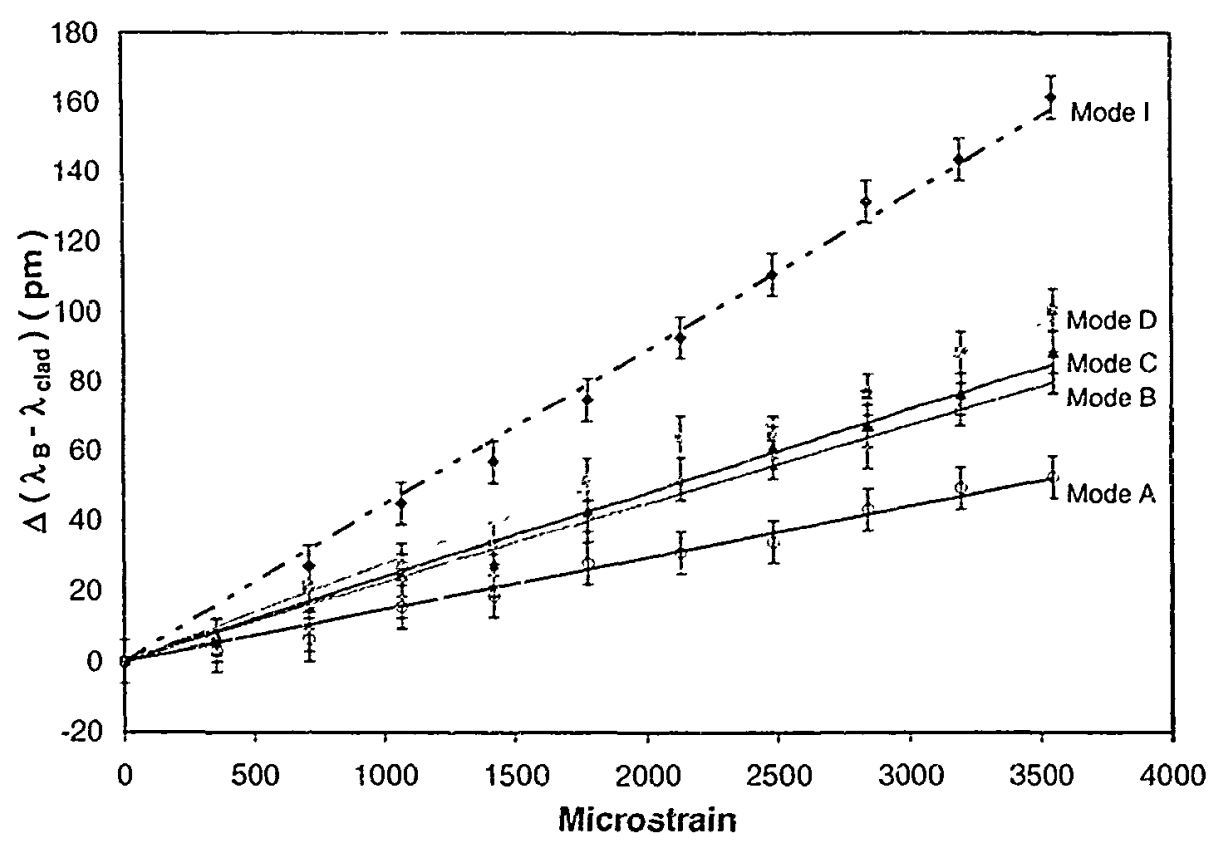

Figure 6.8: The relative wavelength shifts of cladding mode resonances relative to the Bragg resonance under axial strain perturbations.

For SMF-28 fibre, the strain sensitivity of the Bragg rescinance is $10 \%$ smaller than that obtained here, i.e. $1.082 \mathrm{pm} / \mu \varepsilon$ instead of $1.166 \mathrm{pm} / \mu \varepsilon$ (for the core mode). However, when we plot the differential strain sensitivity of the cladding mode resonances relative to the Bragg resonance (i.c. the slopes of Fig. 6.8) as a function of the wavelength distance from Bragg resonance (Fig. 6.9), we get the same linear dependence as was observed in SMF-28 fibre, but with values consistently lower by a factor of about 2. This difference arises because of the higher refractive index contrast between the core and air-hole region in the PCF fibre which confines more of the fields of the cladding modes in the libre core area, especially in the doped region. This reduces the differential strain sensitivity between core and cladding modes compared with SMF-28 fibre where 
the core mode propagates in the germanium-doped region while the cladding modes have most of their energy localized in the silica cladding.

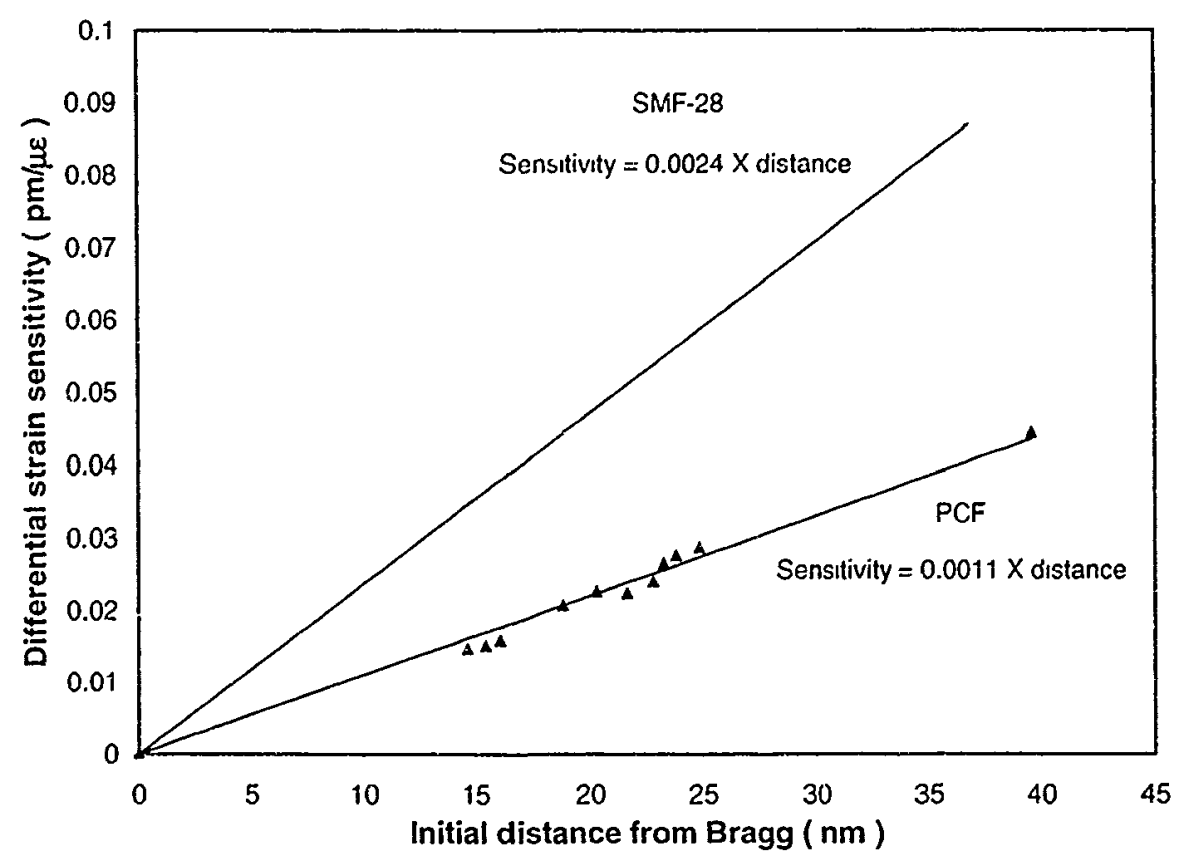

Figure 6.9: The strain sensitivity differences of core and cladding modes in the PCF fibre and SMF-28 fibre

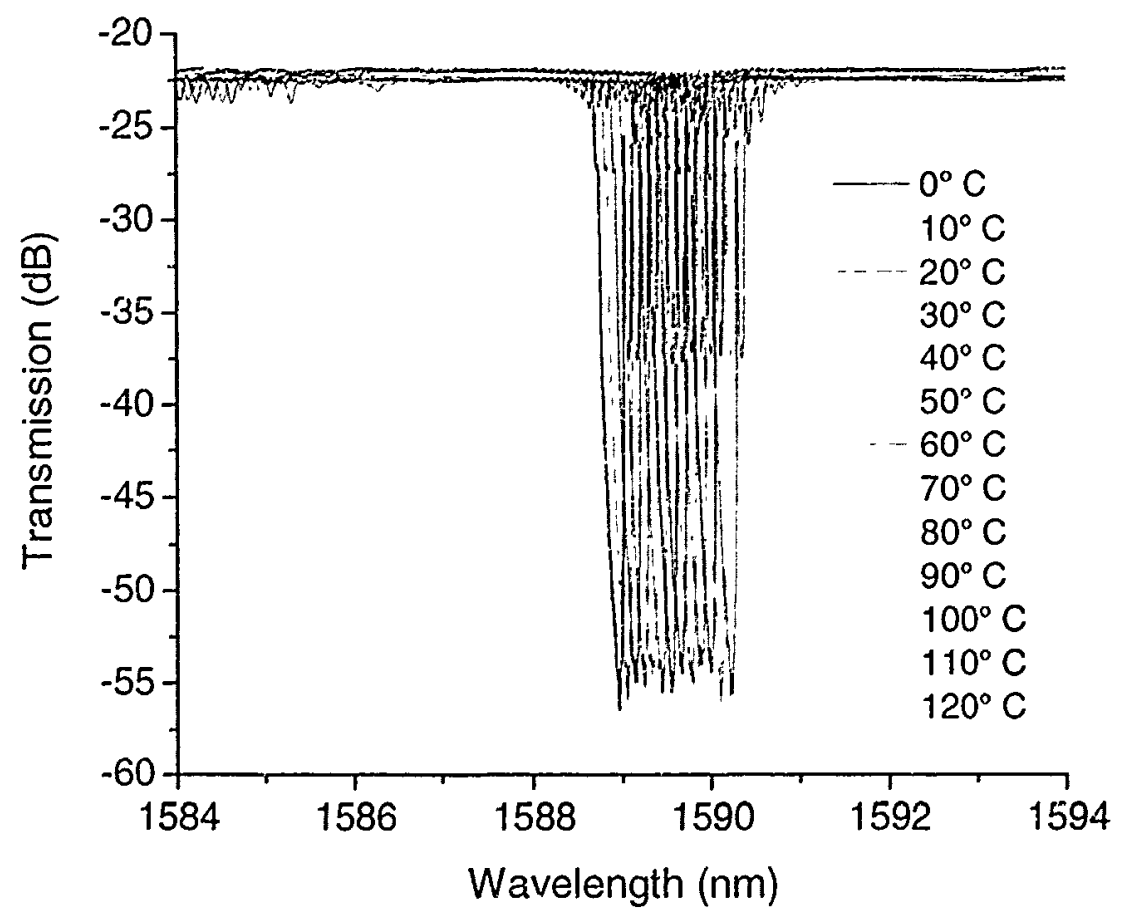

Figure 6.10: The core mode resonance shift under temperature perturbations 


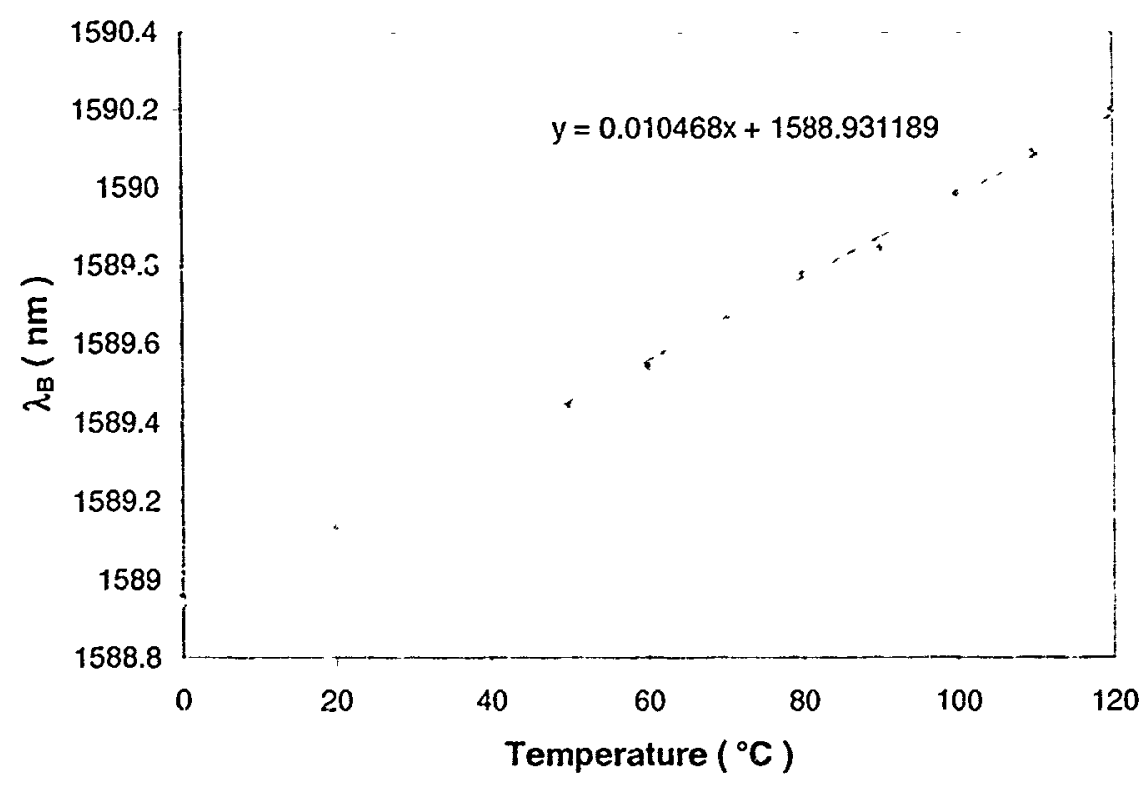

Figure 6.11: The temperature sensitivity of the core mode

Similarly to strain, the effect of temperature on our FBG-PCF resonances was measured from $0{ }^{\circ} \mathrm{C}$ to $120^{\circ} \mathrm{C}$ in a temperature-controlled oven by tracking the individual resonance peak shifts as well as the differential temperature sensitivity (still relative to the Bragg resonance). Fig. 6.10 and Fig. 6.11 show the core mode resonance shift with the temperature perturbations and the core mode temperature sensitivity respectively. The results are given in Table 6.2. For comparison, the temperature sensitivity of the Bragg resonance in SMF-28 fibres $\left(9.579 \mathrm{pm} /{ }^{\circ} \mathrm{C}\right)$ is less than that obtained here $\left(10.47 \mathrm{pm} /{ }^{\circ} \mathrm{C}\right)$. In terms of differential sensitivity, Fig. 6.12 shows the wavelength shifts of the cladding mode resonances relative to the Bragg resonance from $0{ }^{\wedge} \mathrm{C}$ to $120^{\circ} \mathrm{C}$. The data shows a general downward trend (with slope of the order of $0.2 \mathrm{pm} /{ }^{\wedge} \mathrm{C}$, and superimposed random deviations not much greater than our estimated 
measurement accuracy), a similar behavior to what was observed in SMF-28 fibres ( \pm 10 pm between -9 and $+70^{\circ} \mathrm{C}[53]$ ). Between 0 and $80^{\circ} \mathrm{C}$, the relative cladding mode shifts vary between -12 and $+8 \mathrm{pm}$ which is equivalent to an unccrtainty of $\dot{ \pm} 500 \mu \varepsilon$ for a strain measurement with mode I if the temperature is not known (i.e. for a temperature "blind" measurement in an environment where the temperature lies in that range).

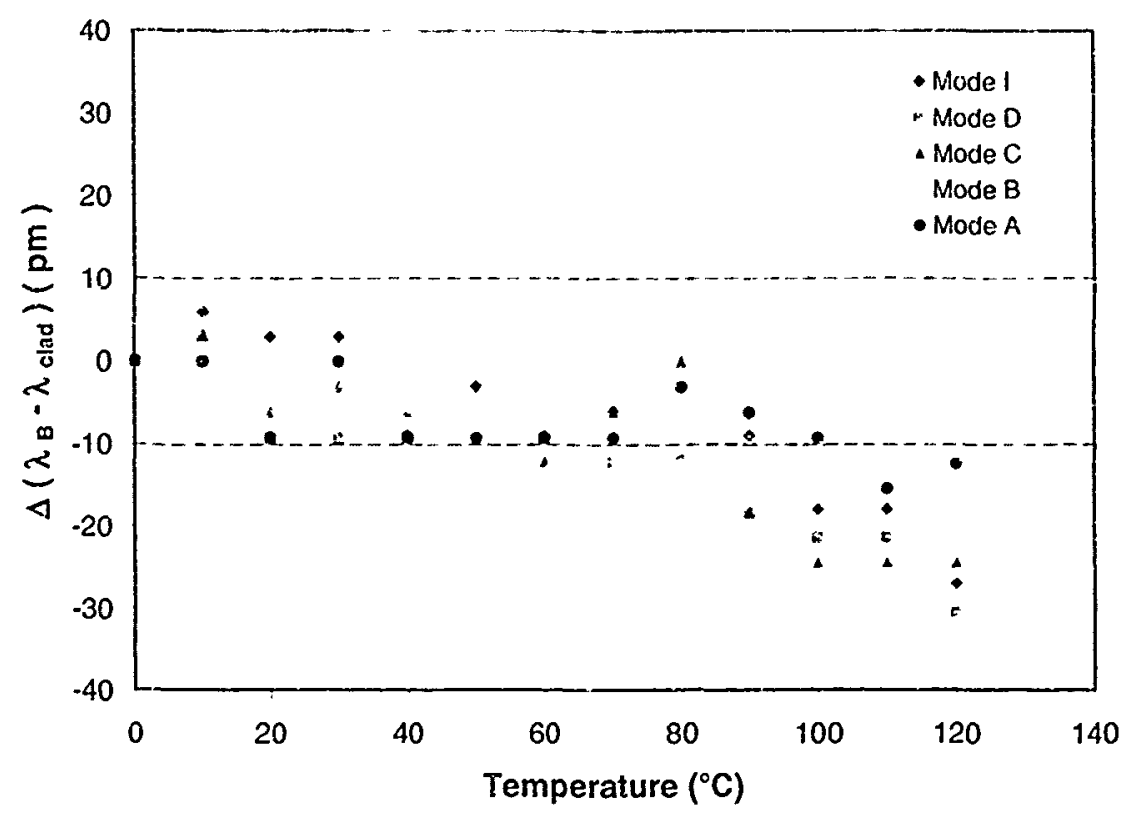

Figure 6.12: The relative wavelength shifts of cladding mode resonances relative to the Bragg resonance of $\mathrm{FBG}$ under temperature perturbations. 


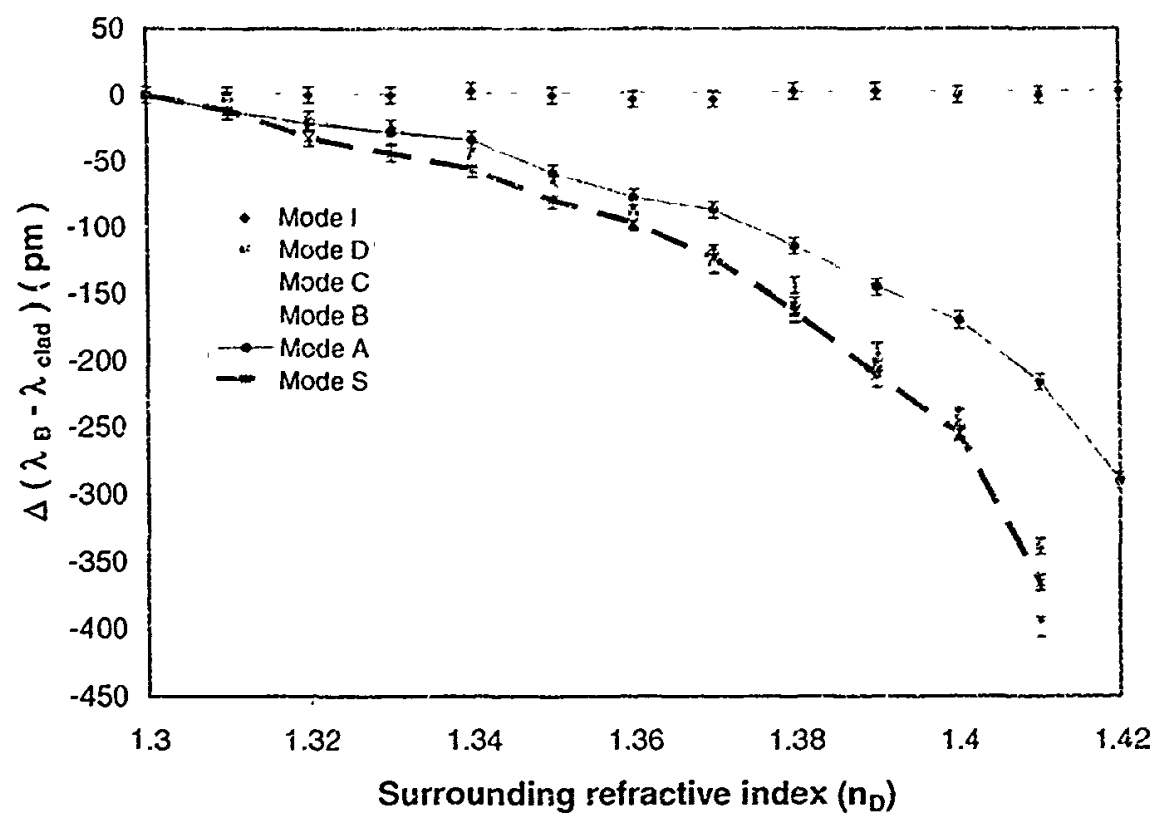

Figure 6.13: The relative wavelength shifts of cladding mode resonances relative to the Bragg resonance, with respect to surrounding refractive index $\left(n_{1}\right)$ changes.

\subsubsection{Surrounding Refractive Index Sensitivity}

Finally, another very widespread application of cladding mode-based fibre sensors is refractometry, since by definition the cladding mode resonances are sensitive to SRI. Out of the large body of literature on this topic, some representative exampies include sensors bascd on tilted FBGis $[23,52]$, long period gratings (LPG) [71], and cladding-reduced FBGs [12]. An additional possibility only available in PCF fibres consists of filling the ai--holes with a gas or a liquid, thereby increasing the overlap between the perturbation and the cladding modes (and even between the corc mode evanescent field in the air holes) [72]. The sensitivity of those sensors can be very high because of the strong field penctration in the medium filling the air-hole region, but it is somewhat difficult to get the material to be sensed to actually penetrate the holes [73]. In general, LPGs and 
TFBGs have different advantages and drawbacks but an important difference lies in the fact that while LPGs can be at least two orders of magnitude more sensilive than TFBGs, they are not necessarily more accurate because of a lower Q-factor (the wavelength of the resonance divided by width of the resonance) [74], and strong cross-sensitivities to multiple kinds of perturbations (strain, temperature, bending, etc..) [75]. Here we concentrate our efforts in measuring the SRI sensitivity of the cladding mode resonances to media located outside the fibre diameter. In the experiment, the grating was inmersed in calibrated refractive index liquid oils from Cargille Corporation: the refraciive indices listed $\left(\mathrm{n}_{\mathrm{D}}\right)$ are the exact values at a wavelength of $589 \mathrm{~nm}$ (the supplier provides an equation to estimate the values of the refractive indices at the varelengths we used but this equation has a listed uncertainty of 0.001 ).

Fig. 6.13 shows the cladding resonance wavelength shifts due to surrounding refractive index changes. These absolute shifts are equivalent to differential wavelength shifts (relative to the Bragg resonance) since the Bragg resonance is totally immune to SRI. Therefore, apart from the $\pm 20 \mathrm{pm}$ uncertainty noted above, using waveiength differences allows to perform refractometric measurements that are independent of temperature. An interesting feature of Fig. 6.13 is the fact that mode $I$ is also insensitive to SRI changes, as expected from the simulaied mode profile of Fig. 6.3 (SRI independent modes were previously identified in [28]). Othei cladding mode resonances behave as usual and their sensitivity increases when the cladding mode approaches its cutoff (i.e. effective index $=$ SRI). For comparison, Fig. 6.13 also shows a cladding mode (mode $S$ ) response for a SMF-28 fibre. The resonance wavelength of mode $S$ is located 
$23.8 \mathrm{~nm}$ from the Bragg wavelength, the same distance as mode $D$ in our PCF fibre. It can be seen that the index sensitivity of mode $D$ and mode $S$ are almost the same, which means that $\partial \mathrm{n}_{\mathrm{eII}} / \partial \mathrm{n}_{\mathrm{SR} I}$ of the cladding mode involved in both fibres is almost the same even though the effective index and absolute wavelengths are different. The measurement range for SRI is similar for both types of fibre, from 1.3 to 1.42 but the great advantage of the PCF is that a single cladding mode can be followed over many nanometers without ambiguity from neighbouring modes. In $\mathrm{SMF}-28$ fibres the cladding modes are separated by $400-1000$ pm, while here, as can be seen from Fig. 6.2 if a $-5 \mathrm{~dB}$ threshold is used for mode finding, mode $B$ can be unambiguously followed over a $5000 \mathrm{pm}$ window and mode $D$ for over $15000 \mathrm{pm}$. The best sensitivity reaches $\sim 12 \mathrm{~nm} / \mathrm{RIU}$ (RIU=refractive index units) in SRI ranges where each mode approaches cut-off. Given a detection system with a resolution of $6 \mathrm{pm}$ (for the wavelength difference between the Bragg and the cladding mode used) this corresponds to a detection limit of $5 \times 10^{-4}$ RIU.

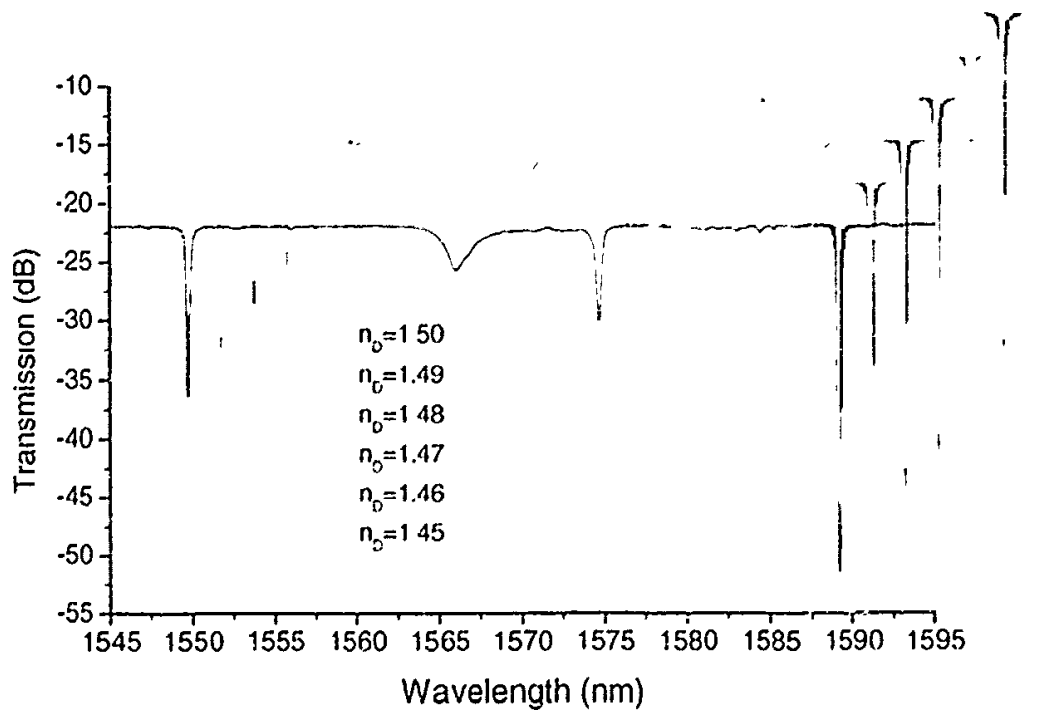

Figure 6.14: The transmission spectra of FBG-PCF immersed in higher index oil. 
Fig. 6.14 shows the FBGs in PCF immersed in oils that have refractive indices higher than the cladding index $\left(n_{I}=1.45 \sim 1.50\right)$. While the cladding modes $B, C$ and $D$ show the typical radiation mode characteristics as discussed in Chapter 5, the cladding mode A still has the narrow resonance and the resunance peak doesn't change with SRI changes. This cladding mode A behaves similarly with the ghost mode of a TFBG in conventional SMF-28 fibre in higher SRl, but the mode A resonance peak shifts with the low SRI as shown in Fig. 6.13, in contrast to the ghost mode in SMF-28 fibre. So if we recoat the PCF using e coating matcrials with a refractive index higher than the refractive index of silica, unlike the cladding mode resonances (except ghost mode resonance) of TFBG in SMF-28 fibre that will disappear or dramatically increase their resonance bandwidth, there are three resonances in PCF which have narrow resonances: the core mode resonance, as well as the Mode A and Mode I resonances SRI insensitive in the transmision spectrum. By tiacking those three resonance wavelength shifts, it is possible to remove the system measurement errors, a more accurate ismperature and SRI insensitive strain sensor can be made.

\subsubsection{Bending Sensitivity}

Bending a fibre can significantly increase the fibre loss due to the core mode coupling to radiation modes and even the core mode itself can be radiate the light, the bending increases the polarization dependent loss as well, because it increases the asymmetry of th: gratings written in fibre core and also changes refrative indices of core and cladding layers in the inner and outer of the bended regien as a push-pull effect as the fibre has the different stain and stress in the cross section. The transmission characteristics FBGs in 
PCF with different bending radius is measured and shown in Fig. 6.15. From Fig. 6.15 it can be seen that the core and cladding mode resonances change with the different bending radius. Mode $\mathrm{B}$ and Mode $\mathrm{D}$ show large amplitude changes and the Mode $\mathrm{C}$ shows a large wavelength shift at the short wavelength end.

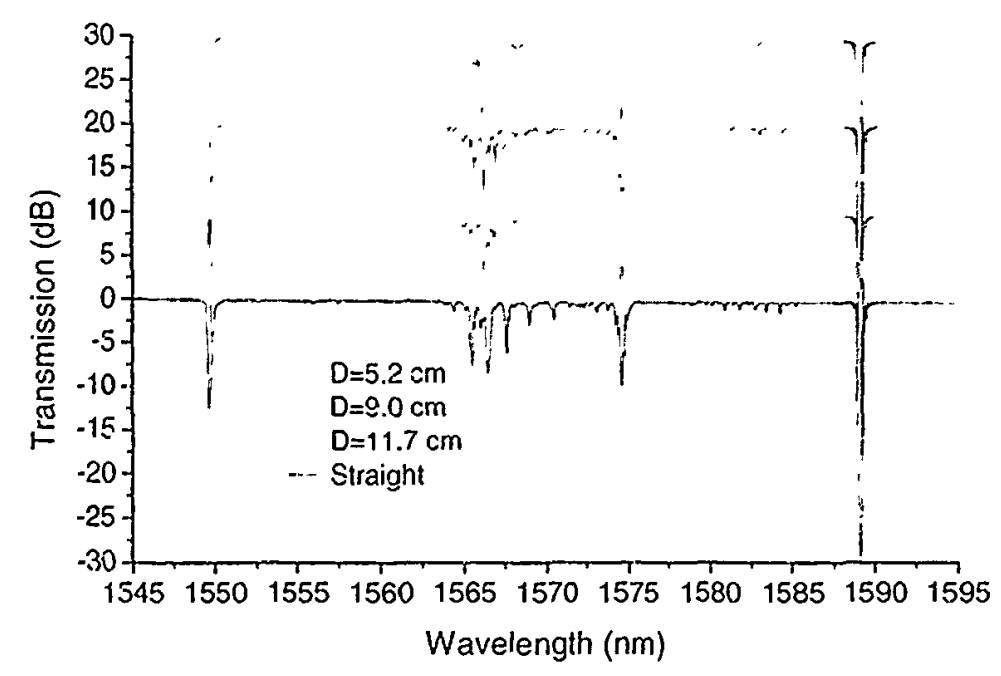

Figure 6.15: Thr transmission spectra of the FBGs in PCF with different bending diameters

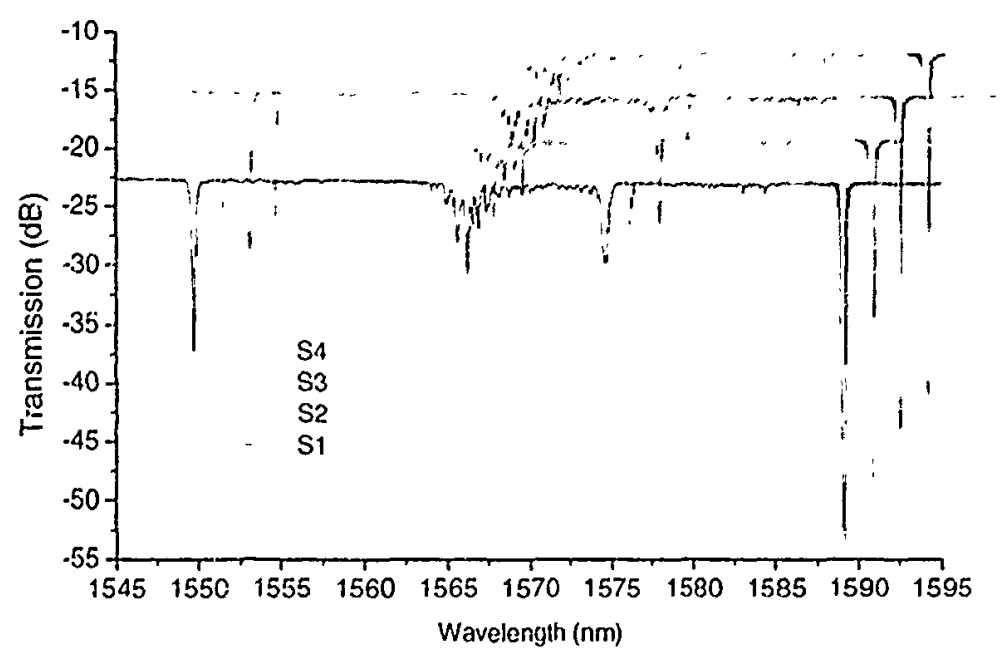

Figure 6.16: The bended $(\mathrm{D}=9 \mathrm{~cm}) \mathrm{FBGs}$ in $\mathrm{PCF}$ with different polarization states 
Fig. 6.16 shows the different polarization states of the FBGs in PCF with the bending diameter of $9.0 \mathrm{~cm}$. The 4 polarization states of Mode A resonance show large different resonance shapes and amplitudes. As discussed before, the polarization states shown in Fig. 6.16 might not be the best representation of the maximum differences between the polarization states. Further polarization dependence measurement needed to further explore the characteristics of the FBGs in PCFs with different bending radius.

\subsection{Discussions}

The PCF modes in this work show some unique characteristics which has not reported before. First there are several cladding modes such as Mode A to Mode D, that show a similar SRI sensitivity as the cladding modes in SMF-28 fibre; secondly Mode I is not sensitive to the SRI changes but it is about $40 \mathrm{~nm}$ aw ay from the core mode resonance, in contrast with the work done by Dr. Eggleton's group in 1999 [28]. The Fig. 6.17 shows the cross section of the PCF and the transmission spectra of FBGs in PCF in the air and higher SRI 1.457. Here we can see from their work that all the cladding mode resonances are very close to core mode resonances. In their PCF, the air-hole djameter $\mathrm{d} \sim 2 \mu \mathrm{m}$, spacing period $\Lambda \sim 10 \mu \mathrm{m}$ and doped core $\mathrm{d}_{\llcorner} \sim 2 \mu \mathrm{m}$. The major difference of our PCF with their PCF is the spacing period. The spacing period of our PCF is $\sim 3.85 \mu \mathrm{m}$, which is less than half of their spacing period. So we can control the mode resonance characteristics by designing the PCF with the different air-hole and doped core diameters and the spacing period for specific sensor applications. 


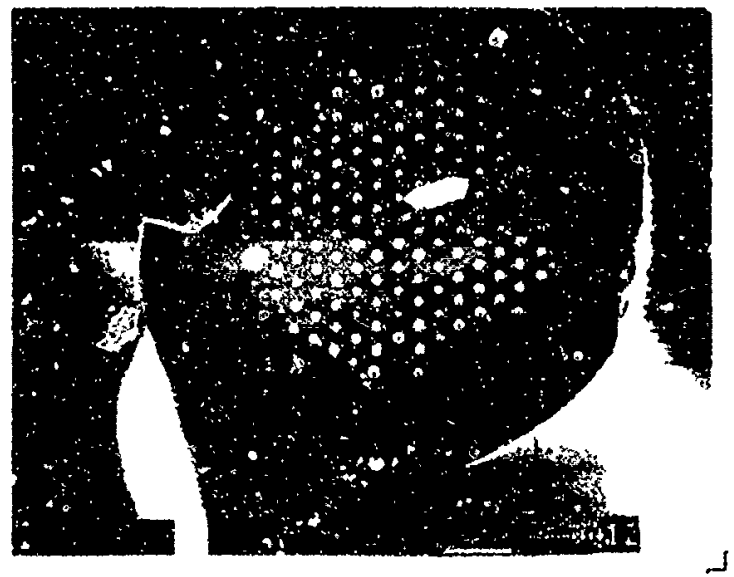

(a)

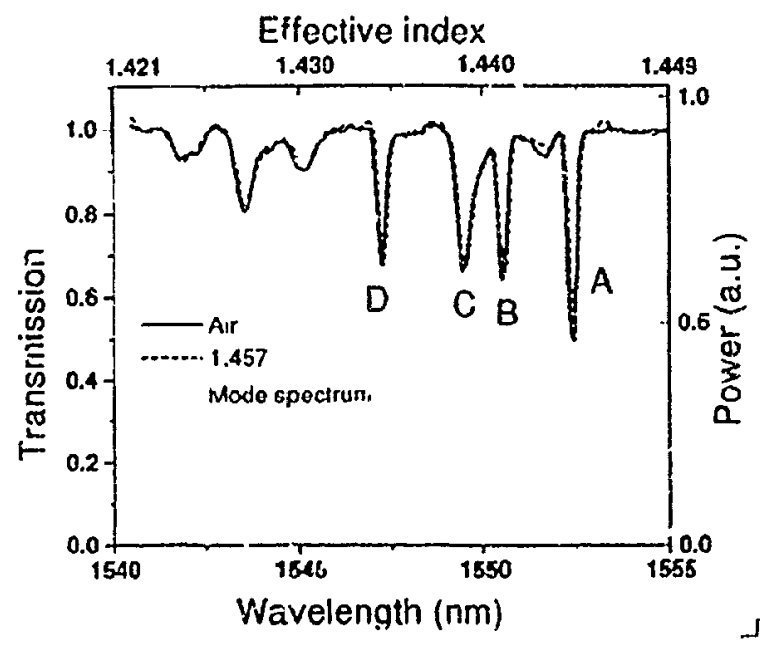

(b)

Figure 6.17: (a) The PCF fibre cross section [28]; (b) The spectra of FBGs in PCF [28].

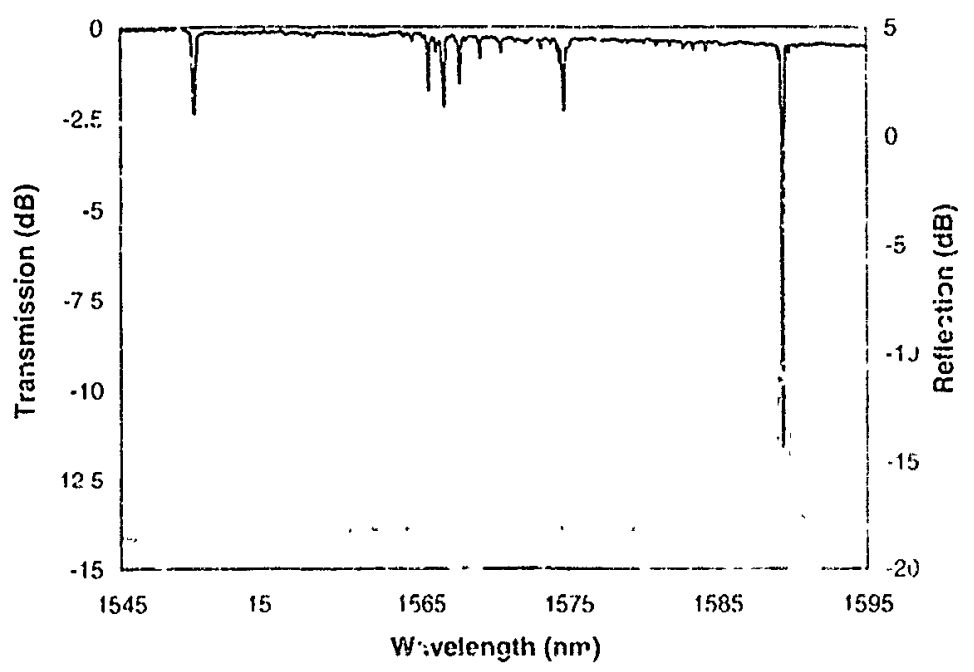

Figare 6.1: ; he transmission and reflection spectra of a $1 \mathrm{~cm}$-leng TWBG in PCF fiore. 
Fig. 6.18 shows the transmission and reflection spectra of a TFBG with $2^{\circ}$ tilted angle in PCF. Comparing with the Fig. 6.2, it is clearly shown that a small tilt angle would not help the core to cladding modes couplings in PCF. This is totally different from TFBGs in SMF-28 fibre we discussed in Chapter 5. Except the large cross section difference between the PCF and SMF-28 fibre, the main reason why a small tilted angle would not increase the core and cladding mode couplings is that the PCF has a much smaller doped core $(1.9 \mu \mathrm{m}$ instead of $8.3 \mu \mathrm{m})$ doped core. The small doped fibre core makes the core mode no longer a conventional core mode with an effective index between the cladding refractive index and the core refractive index. Now the effective index of the core mode is less than the refractive index of the silical outer cladding. If this was in SMF, then the core mode would decay to the low order cladding modes and the fields could reach to the cladding/air boundary. But in the PCF, the effective index of the core mode is less than refractive :adex of the silica, but there is the air-hole layer where the average refrative index is also much less than the silica. So the core mode now is guided by the sm." doped core and solid silica core. This reduces the energy confinement in doped core region of PCF, the confinement factor is $0.1 \mathrm{i}$ 1 doped core region of PCF instead of 0.8 in doped core region in SMF-28 fibre, bul the small doped core of PCF increases the core mode to cladding iodes couplings, because core and cladding modes now have more similar field distributions in the small doped core res. on especiall, for high order cladding modes (compared with conventional large doped core). Finally when the core and cladding modes, hatre similar field distrbutions in the small doped core, a tilt angle of the gratting plane won't heip increasing the core and cladding mode couplings. It can be 
seen from the core to core mode coupling of the TFBG in SMF 28 fibre, when a tilted angle is added. the core mode resonance amplitade reduces when the same index modulation is applied.

\subsection{Summary}

In this chapter. strong FBGs are written in a germanium/lluorine co doped core, hydrogen-loaded PCF fibre with $193 \mathrm{~nm}$ ArF excimer laser light and a phase mask. A limited number of strong cladding mode resonances are obtained with an un-tilted FBG, in contrast to conventional fibres where a large number of strong cladding mede resonances can be obtained, but only whe "he grating planes are tilted. The strain. temperature and refractive index sensitivities of core and cladding mode resonatices in this PCF fibre have been determined experimentally. In addition to the uswal core and cladding niodes, this PCF fibre has a cladding mode whose optical ficld is confined by the air-hole region, making this mode insensitive to the surrounding reftiktive iridex changes, just like the cure-guided mode. Like in conventional fibres, the cladding mode resonances in PCF fibre show very small differential temperature sensitivity relative to the Bragg resonance, less than $0.2 \mathrm{pm} /{ }^{\circ \mathrm{C}}$. Given this characteristic, making differential measurements of the wavelength of Individual resonances with respect to the Bragg warelength will be relatively independent of temperature. On the whe: hand, the differentiai strain sensitivity of the cladding mode resonances relttive to :he Bragg resonance increatses linearly with the initial distance of each teronance from the Bragg resonance. This differential strain sensitivity of the cladding mode resonances in our PCF fibre turns sut to be a factor of 2 smaller than in SMF-28 fibre. We explain this difference 
by the larger confinement of the cladding modes studied in the core region of tine PCF fibre (due to the air-holes), which reduces the difference in the photo-elastic effect seen by the cladding modes, relative to the core modes. Finally, apart from the air hole confined mode (mode I) the refractive index differential sensitivities of the cladding modes behave exactly like the modes of conventional libre: the wavelength shift per RIU converge to values around $10 \mathrm{~nm} / \mathrm{RIU}$ as the modes approach cut-off in buth types of fibres. The main advantage of the PCF fibr short period gratings in sensing applications resides in the presence of the SRI-insensitive mode I, whose differential resonance shift zan be used to sense axial strain independently of temperature and regardless of the material in which the sensor is placed, including liquids, epoxies or structural materials steh as concrete. While the individual sensitivitics of our sensor do not match the best values obtained with optimized single-parameter sensors, we feel that the multiparameter sensing capability, very low cross-sensitivity to temperature, and simple sensor construction is a distinct advaniage. 


\section{Chapter 7}

\section{Conclusions and Future Work}

\subsection{Original Contributions}

In this thesis work, novel sensors based on TFBGs in conventional SMFs have been developed. By tracking the differential core and cladding mode resonance wavelength shifts, temperature-insensitive strain, refractive index and vibration sensor can be made. We were the first in the world to introduce the algorithm of tracking the differential sensitivity of temperature, strain and index in weakly TFBG and realize the temperature insensitive characteristics in SMFs. The effect of the fibre cladding diameter thickness for strain, temperature and index sensitivities has been explored experimentally.

A fast and accurate numerical method has been developed to analyze the fibre modes in multilayer step-index fibres. New analytical inverse transfer matrices are derived which could dramatically improve the calculation accuracy and efficiency, and the analytical derivatives for the dispersion equations were derived as well. Based on those, the Cauchy's Integration Method is used to solve the fibre modes in multilayer step-index fibres with lossy and gain media. This numerical method is successfully used to solve fibre modes in fibre structures for our sensor applications.

Now formulations for calculating the liybrid mode coupling in mulatilayer step-index fibres with lossy and gain media have been derived for FBGs with and without a tilted 
angle. The specific transmission spectrum characteristics of TFBG in SMF-28 fibre with a thin gold coating are well explained by our simulation results using the new formulations we developed.

Finally, strong FBGs have been written in Photonic Crystal Fibre. New cladding modes in PCF that are sensitive to the surrounding refractive index changes have been found, and they were not reported before. By tracking the differential core and cladding mode resonance wavelength shifts, temperature-insensitive strain, refractive index and vibration sensor can be made. As cladding modes in PCF are a combination of index sensitive and insensitive modes, therefore FBG in PCF can be used as a multi-parameter temperature insensitive strain and index sensor. By embedded FBGs in construction materials such as concrete, real time monitoring the strain and index changes can be realized.

\subsection{Significance of Work}

Our research work for the TFBG brought us a lot of attention recently in the world as the TFBG takes the advantage of temperature insensitive characteristics. More re,earch groups now involve in TFBGs especiaily for the TFBG in the fibre with a thin metal coating.

Our work for FBG in PCF brings a different view for PCF in sensor applications. By designing the PCF with different hole sizes and periods, specific cladding modes which are interesting for sensor applications will exist in PCF and can be excited by FBGs.

the numerical method developed for analyzing vectorial modes in multilayer stepindex fibre can not only be used to analyze fibre modes in our SMFs, but also it is a 
general numerical methods can be used analyze other fibres such as gain fibre for fibre laser application., Bragg fibres and hollow fibres as well. Based on our numerical method developed for an lyzing vectorial modes, a fast and accurate fibre mode solver can be developed for a commercial CAD purpose.

The mode coupling analysis by using full vectorial modes also provides a good tool to analyze the FBG and TFBG in fibres with lossless, lossy and gain media. It is for the fibre coupling in core region, but ${ }^{\circ} t$ can easy extend to mode couplings in cladding layers for more general applications.

The significant of this work also can be seen from our publications: it generated one international patent application, more than 20 refereed journal and conference papers and one book chapter as shown in Appendix B.

\subsection{Future Work}

The future work can be focused on following areas: (i) for the mode in multilayer optical fibres, more work to analyze leaky modes and radiation modes is needed. This can bring a better understanding the mode characteristics when a TFBG is immersed in liquids. Comparing with the guided modes, leaky modes and radiation modes are more difficult to solve as now their fields are no longer converging in the surrounding medium, so it is impossible to normalize the mode power to $\mathrm{I} \mathrm{W}$, and that wuald bring a problem for the mode coupling calculations.

(ii) For the core mode and cladding mode couplings in TFBG, coupling constants for the corc mode to leaky modes and radiation modes need to be calculated. Here the core mode and leaky mode couplings are very important for the fibre with a thin gold coating, 
in which the leaky mode resonances show very specific transmission spectrum features. Further work also can be done for simulating the transmission spectrum of TFBG using the Runge-Kutta method.

(iii) For TFBG sensors, firstly we can look how to make real applications for the sensors we developed such as using the vibration sensor in airplane wings, the vibration sensor in war vehicles and index sensor for biumaterial testing etc. Other that that, novel sensors based on tracking leaky mode resonances in the TFBG of fibre with a thin gold coating can be explored much more as well as how to track the plasmon mode resonance directly, because the plasmon mode resonances show the biggest sensitivity for index sensing.

(iv) Finally, for PCF fibres we see that the different hole size and period in PCF structures can affect cladding mode sensitivity for SRI, therefore more work can be done by designing or using the different PCFs to further explore the index sensing characteristics in PCFs. We can also directly use the FBG in PCF for a real multiparameter sensor to monitor the structure health for the temperature insensitive strain and index perturbations. 


\section{Bibliography}

[1] J. Senior, Optical fiber communications, McGraw Hill, 2000

[2] V. Vali and R.W. Shorthill, "Fiber Ring Inteferometer", Appl. Opt., vol. 15, pp. 1099-1100, 1976.

[3] K. O. Hill, Y. Fujii, D. C. Johnson, and B. S. Kawasaki, "Photosensitivity is optical fiber waveguides: Application to reflection filter fabrication," Appl. Phys. Let!., vol. 32. pp. 647$649,1978$.

[4] G. Meltz, W. W. Morey, and W. H. Glenn, "Formation of Bragg gratings in uptical fibers by a transverse holographic method," Opt. Lett. vol. 14, pp. 823-825, 1989.

[5] K. O. Hill, B. Malo, F. Bilodeau. D. C. Johnson, and J. Albert. "Bragg gratings fabricated in monomocie photosensitive optical fiber by UV exposure through a phase mask," Appl. Phys. Lett. vol. 62, pp. 1035-1037, 1993.

[6] A. Othonos and K. Kalli, Fiber Bragg Gratings: Findamentuls and Applations in Telecommunications and Sensing, Boston: Artech House, 1999.

[7] R. Kashyap, Fiber Bragg Gratings, San Diego: Academic Press, 1999.

[8] H. Sheng, M. Fu, T. Chen, W. Liu, and S. Bor, "A lateral pressure sensor using a fiber Bragg grating," IEEE Photon. Technot. L_ett., vol. 16, pp. 1 ;46-1148, 2604.

[9] X. Shu, Y. Lir, D. Zhao, B. Gwandu, F. Floreani, L. Zhang, and I. Bernicn, "Dependence of temperature and strain coefficients on fiber grating type and its application to simultaneous temperature and strain measurement," Opt. Lett., vol. 2'T, pp. 701-703, 2002.

[10] S. Baek, Y. Jeong, and B. Iees, "Characteristics of short-period blazed fiber Bragg gratings for use as macro-bendling sensors," 1 ppl. Opt. vol. 41, pp. 631-636, 2002.

[11] A. Martines, Y. Lai, M. Dubov, L. Y. Khrushchev, and I. Bemion, "Y'se!or bendirg sensors based on fiber Bragg Gratings inscribed by infrared feintosecond laser," Elcctren. Lett., wi 41 , pp. 472-474, 2005.

[12] A. Iadicicco, A. Cusano. A. Cutolo, R. Berini. and M. Giordano, "Thimed fiber Brags" gratings as high sensitivity refractive index .ensor," iElit: Photon. T'c hnol. Lett., vol. 16, pp. 1149-1151, 2004. 
[13j A. Indicicco, S. Campopiano, A. Cutolo, M. Giordano, and A. Cusano "Microstructure fiber Bragg gsalings: analyss and fabrication," Eiectron. Letl., vol. 41. pp. 466-468, 2005.

[14] C. Chen, L. Xieng, A. Jafari, and J. Albent, "Differential sensitivity characteristics of tilted fiber Bragg grating sensors". Proc. SPIE 6004, 60040B1-10, 2005.

[15] C. Tsao. Optical Fiber Waveguide Analysis, Oxford: New York, 1992.

[16] C Yeh and G. Lindgien, "Computing the propagation characteristics of radially stratified fibers: an efficient method", Appl. Opt. vol. 16(2) pp. 483-493, 1977.

[17] J. Chilwell and I. Hodgkinson, "Thin-films field-tiansfer matrix theory of planar multilayer waveguides and reflection from prism-luaded waveguide," J. Opt. Soc. Amer. A, vol. 1, pp. $742-753,1984$.

[18] S. Dods, "Technical Backgound: Faher Mode Solvers", Optiwave Corporation, 2006.

[19] A. Yariv, "Coupled-mode theory for guided-wave optics," IEEE J. of Quantum Electron., vol. QE-9, pp. 919-933, 1973.

[20] T. Erdogan and J. E. Sipe. "Tilted fiber phas gratings," J. Opt. Soc. Am. A vol. I3, pp. 296$313,1997$.

[2i] T. Erdogan, "Cladding-mode resonances in short- and long period fiber grating filters," $J$. Opt. Soc. Am. A vol. 14, pp. 1760-1773, 1997.

[22] K. S. Lec, and T. Erdogan, "Fibes mode coupling in transmissive and reflective tilted fiber gratings," Appl. Opt.. vol. 39, pp. 1394-1404, 2000.

[23] G. Laffont, and P. Ferdinand, "Tilted short-period fiber-Bragg-grating-induced coupling to clacifing modes for accurate refractometer,' Meas. Sci. and Technol., 12, pp. 765-770), 2001.

[24] G. Laffont, and P. Ferdinand, "Sensitivity of fiber Bragg grating to external refractive index higher than that of silica," Electron. Let1. vol. 37, pp. 289-290, 2001.

[25] J. C. Knight, T. A. Birks, P. St. J. Russcll, and D. M. Atkin. "All-silica single-mode optical fiber with photonic crysial cladding," Opt. Lett. vol. 21, pp. 1547-1549, 1996.

[26] P. Russell. "Photonic crystal fihers," Science, vol. 299, pp. 358-362. 2003.

[27] J. C. Hanagan, R. Amezcua-Corrca, F. Polctti, J.R. Hayes, N.G.R. Broderick, and D. I. Ric'sardson. "Parasitic modes in large mode area microstructuned libers." in Optic al Fibe" Communication Conference. Technical Digest (OSA), paper (OML4. 2007.

[28] B. J. Eggleton, P. S. Westbrook, R. S. Windeler. S. Spalter, and T. A. Strasser, "Grating resonances in airsilica microstructured optical fibers," Opt. I., It. Wol. 24, pp. 1460-1462. 1999. 
[29] V. Beugin, L. Bigot, P. May, M. Lancry, Y. Quiquempois, M. Douay, G. Melin, A. Fleureau, S. Lempereur; and L. Gasca, "Efficient Bragg gratings in phosphosilicate and germanosilicate photonic crystal fiber," Appl. Opt. vol. 45, pp. 8186-8193, 2006.

[30] N. Groothoff, J. Canning, E. Buckley, K. L.yttikainen, and J. Zagari, "Bragg gratings in airsilica structured fibers," $O_{p t}$. Lett. vol. 28, pp. 233-235, 2003.

[31] S. J. Mihailov, D. Grobnic, H. M. Ding, C. W. Smelser, and J. Broeng, "Femtosecord IR laser fabrication of Bragg gratings in photonic crystal fibers and tapers," IEEE , hoton. Technol. Lett. vol. 18, pp. 1837-1839, 2006.

[32] L. B. Fu, G. D. Marshall, J. A. Bolger, P. Steinvurzel, E. C. Magi, M. J. Withford, and B. J. Eggleton, "Femtosecond laser writing Bragg gratings in pure silica photonic crystal fibers," Electron. Lett. vol. 41, pp. 638-640, 2005.

[33] G. D. Marshall, D. J. Kan, A. A. Asatryan, L. C. Botten, and M. J. Withford, "Transverse coupling to the core of a photonic crystal fiber: the photo-inscription of gratings," Opt. Express, vol. 15, pp. 7876-7887, 2007.

[34] H. R. Sorensen, J. Canning, J. Laegsgaard, K. Hansen, and P. Varming, "Liquid filling of photonic crystal fibers for grating writing," Opt. Commun., vol. 270, pp. 207-210, 2007.

[35] A. Yariv, Optical electronics in modem communications, Oxford: New York, 1997.

[36] E. Anemogiannis, and E. N. Glytsis, "Multilayer waveguides: efficient numerical analysis of general structures", J. Lightwave Technol., vol. 10, pp. 1344-1351, 1992.

[37] C. Chen, P. Berini, D. Feng, S. Tanev. and V. Tzolov, "Efficient and accurate numerical analysis of multilayer planar optical waveguides in lossy anisotropic media," Opt. Express, vol. 7, pp. 260-272, 2000.

[38] N W. McLachlan, Bessel Functions for Lingineers, Oxford: Clarendon, 1955.

[39] G. E. Keiser. Optical Fiber Corrmunications, McGraw-Hill, 1999.

[40] W. H. Press, S. A. T'ukolsky, W. T. Vetterling and B. P. Flannery, Numerical Recipes in C, Second Edition, Cambridge, 1994.

[41] L. M. Delves and J. N. Lyness, "A numerical method for locating the zeros of an analytic function," Math. Comp., vol. 21, pp. 543-560, 1967.

[42] J. W. Brown and R. V. Churchill, Complex L'ariables and Applications, Sixth Edition, New York: McGraw-Hill, 1996.

[43] OptiGrating 4.3, Optiwave Systems Corporation.

[44] Y. J. He, Y.-L. Lo, J. F. Huang, “Optical-fiber surface-plasmon-resonance sensor employing long-period fiber gratings in multiplexing”, J. Opt. Sor. Am. B, vol. 23, pp. 801-811, 2006. 
[45] צ. Shevchenko and $J$. Albert, "Plasmon resonances in gold-coated tilted fiber Bragg gratings", Opt. Lett, vol. 32, pp. 211-213, 2007.

[46] T. Allsop, R. Neal, S. Rehman, D. J. Webb, D. Mapps, and I. Bennion. "Characterization of infrared surface plasmon resonances generated from a fiber-optical sensor utilizing tilted Bragg gratings," J. Opt. Soc. Am. B, vol. 25, pp. 481-490, 2008.

[47] D. K. W. Lam and B. K. Garside, "Characterization of single-mode optical fiber filters," Appl. Optics, vol. 20, pp. 440-445, 1981.

[48] C. L. Chen, Foundations for Guided-Wav' Optics, John Wiley \& Sons: New Jersey, 2007.

[49] K. Schroeder, W. Ecke, R. Mueller, R. Willsch, and A. Andreev, "A fiber Bragg grating refractometer," Meas. Sci. and Technol., vol. 12, pp. 757-764, 2001.

[50] X. Chen, K. Zhou, L. Zhang, and I. Bennion, "Simultaneous measurement of temperature and external refractive index by use of a hybrid grating in $D$ fiber with enhanced sensitivity by HF etching:" Appl. Opt., vol. 44, pp. 178-182, 2005.

[51] C. Chen, L. Xiong, C. Caucheteur, P. Mégret and J. Albert, "Differential strain sensitivity of higher order cladding modes in weakly tilted fiber Bragg gratings," Proc. SPIE 6379, $63700 \mathrm{E}-\%, 2006$.

[52] C. F. Chan, C. Chen, A. Jafari, A. Laronche, D. J. Thomson and J. Albert, "Optical fiber refractometer using narrowband cladding mode resonance shifts," Appl. Opt., vol. 46, pp.1142-1149, 2007.

153] C. Chen and J. Albert, "Strain-optic coefficients of the individual clacdding modes of a single mode fiber: theory and experiment," Electron. Lett. vol. 48, pp. 1027-1028, 2006.

[54] J. Albert, K. O. Hill, D. C. Johnson, F. Bilodeau, S. J. Mihailov, N. F. Borrelli, and J. Amin, "Bragg gratings in defect-free germanium-doped optical fibers," Opt. Lett. vol. 24, pp. 1266$1208,1999$.

[55] A. Bertholds, and R. Dandliker, "High-resolution photoclastic pressure sensor using lowbirefringence fiber," Appl. Opt. vol. 25, pp. 340-343, 1986.

[56] High resolution swept laser interrogator Model Si720 from Micron Optics Inc. Atlanta, GA, USA.

[57] C. Chen, C. Caucheteur, P. Megret. and J. Albert, "Sensitivity of Tilted Fiber Bragg Grating Sensors with Different Cladding Thicknesses," I8th Conference on Optical Fiber Sensors, paper TuE31, 2006.

[58] A. Cusano, A. Iadicicco, P. Pilla, L. Ccntessa, S. Campopiano, and A. Cutolo, "Cladding mode reorganization in high-refractive index-coated leng-period gratings. effects on the refiactive-index sensitivity." Opt. Lett. vol. 30, pp. 2536-2538, 2005. 
[59] J. Yang, L. Yang, C. Xu, C. Xu, W. Huang, and Y. Li, "Long-period grating refractive index sensor with a modified cladding structure for large operational range and high sensitivity," App. Opt. vol. 45, pp. 6142-6147, 2006.

[60] T. Allsop, F. Floreani, K. P. Jedrzejewski, P. V. S. Marques, R. Romero, D. J. Webb, and I. Bennion, "Spectral Characteristics of tapered LPG device as a sensing element for refractive index and temperature," IEEE/OSA J. Lightwave Technol., vol. 24, pp. 870-878, 2006.

[61] K. W. Chung and S. Yin, "Analysis of a widely tunable long-period grating by use of an ultrathin cladding layer and higher-order cladding mode coupling," Optics Lett., vol. 29, pp. 812-814, 2004.

[62] C. Caucheteur, K. Chah, F. Lhommé, M. Debliquy, D. Lahem, M. Blondel and P. Mégret, "Enhancement of Cladding Modes Couplings in Tilted Bragg Gratings Owing to Cladding Etching," Workshop on Fibers and Optical Passive Components (WFOPC), pp. 234-239, 2005.

[63] T. Guo, A. Ivanov, C. Chen, and J. Albert, "Temperature-independent tilted fiber grating vibration sensor based on cladding-core recoupling." Opt. Lett., vol. 33, pp.1004-1006, 2008.

[64] T. Guo, C. Chen, A. Laronche, and J. Albert, "Power-referenced and temperature-calibrated optical fiber refractometer," IEEE Photon. Technol. Lett. vol. 20, pp.635-637, 2008.

[65] C. Caucheteur, M. Wuilpart, C. Chen, P. Mégret, and J. Albert, "Quasi-distributed refractometer lising tilted Bragg gratings and time domain reflectometry," Opt. Express, vol. 16, pp. 17882-17890, 2008

[66] C. Chen, T. Guo, A. Laronche, and J. Albert, "Radiation mode resonances of tilted fiber Bragg gratings for high index media measurement," 19th Conference on Optical Fiber Sensors ,Proc. SPIE 7004, 700418 pp. 1-4, 2008

[67] S. Maguis, G. Laffont, P. Ferdinand, B. Carbonnier, K. Kham, T. Mekhalif, and M. Millot, "Biofunctionalized tilted Fiber Bragg Gratings for label-free immunosensing," Opt. Express, vol. 16, pp. 19049-19062, 2008.

[68] O. Fra_ão, L. A. Ferreira, F. M. Araújo, and J. L. Santos, "Applications of fiber optic grating technology to multi-parameter measurement," Fiber Integrated Opt. vol. 24, pp. $227-244$, 2005.

[69] B. J. Eggleton, P. S. Westbrook, C. A. White, C. Kerbage, R. S. Windeler, and G. L. Burdge, "Cladding-mode-resonances in air-silica microstructure optical fibers," J. Lightwav'e Technol. vol. 18, pp. 1084-1100, 2000.

[70] C. Chen, L. Xiong, C. Caucheteur, P. Mégret and J. Albert, "Differential strain sensitivity of higher order cladding modes in weakly tilted fiber Bragg gratings," Proc. SPIE 6379, 63790til-7, 2006. 
[71] H. J. Patrick, "Analysis of the resp snse of lung period fiber gratings to external index of refraction," J. Lightwave Technol., vol. 16, pp. 1606-16I2, 1998.

[72] M. C. P. Huy, G. Laffont, V. Dewynter, P. Ferdinand, L. Labonté, D. Pagnoux, P. Roy, W. Blanc, and B. Dussardier, "Tilted fiber Bragg grating photuw ritten in microstructured optical fibre for improved refractive index measurement," Opt. Express, vol. 14, pp. 10359-10370, 2006.

[73] F. M. Cox, R. Lwin, M. C. J. Large, and C. M. B. Corcieiro, "Opening up optical fibres," Opt. Express. vol. 15, pp. 11843-11848, 2007.

[74] I. M. White, and X. Fan, "On the performance quantification of resonant refractive index sensors," Opt. Express, vol. 16, pp. 1020-1028, 2008.

[75] V. Bhatia, "Applications of long-period gratings to single and multi-parameter sensing," Opt. Express, vol. 4. pp. 457-466, 1999. 


\section{Appendix A: The Power in Multilayer Step-Index Fibre}

\section{with Lossy and Gain Media}

The total power carried by each mode can be calculated as following:

$$
P=\frac{1}{2} \operatorname{Re}\left[\int_{0}^{2 \pi} d \phi \int_{0}^{\infty}\left(e_{r} h_{\phi}^{*}-e_{\phi} h_{r}^{*}\right) r d r\right]
$$

Transverse electric and magnetic fields can be obtained from Eqs. (3.44) to (3.46). For convenience in doing integral of (A.1) in each fibre layer, we rcorganize Eqs. (3.44) and (3.45), they can be rewritten as following:

If $n_{\text {edf }}<n_{1}$

$$
\begin{aligned}
& e_{r}=\left[\tilde{A}_{t} J_{v-1}\left(u_{t} r\right)+\tilde{B}_{t} J_{v+1}\left(u_{t} r\right)+\tilde{C}_{i} Y_{v-1}\left(u_{t} r\right)+\tilde{D}_{\imath} Y_{v+1}\left(u_{t} r\right)\right] \exp (j v \phi) \\
& e_{\phi}=j\left[\tilde{A}_{t} J_{v-1}\left(u_{t} r\right)-\tilde{B}_{t} J_{v+1}\left(u_{t} r\right)+\tilde{C}_{t} Y_{v-1}\left(u_{t} r\right)-\tilde{D}_{t} Y_{v+1}\left(u_{i} r\right)\right] \exp (j v \phi) \\
& h_{l}=j\left[\tilde{E}_{t}^{r}\left(u_{t} r\right)+\tilde{F}_{\imath} J_{v+1}\left(u_{i} r\right)+\tilde{G}_{i} Y_{v-1}\left(u_{t} r\right)+\tilde{H}_{i} Y_{v+1}\left(u_{t} r\right)\right] \exp (j v \phi) \\
& h_{\phi}=\left[-\tilde{E}_{t} J_{v-1}\left(u_{i} r\right)+\tilde{F}_{\imath} J_{v+1}\left(u_{i} r\right)-\tilde{G}_{\imath} Y_{v-1}\left(u_{t} r\right)+\hat{H}_{t} Y_{v+1}\left(u_{t} r\right)\right] \exp (j v \phi)
\end{aligned}
$$

Where

$$
\begin{aligned}
& \tilde{A}_{t}=-\frac{\beta u_{t}}{2 \omega \varepsilon_{i}} A_{t}+\frac{u_{t}}{2} C_{t} \\
& \tilde{B}_{t}=\frac{\beta u_{t}}{2 \omega \varepsilon_{t}} A_{t}+\frac{u_{t}}{2} C_{t}
\end{aligned}
$$




$$
\begin{aligned}
& \tilde{C}_{t}=-\frac{\beta u_{t}}{2 \omega \varepsilon_{t}} B_{t}+\frac{u_{t}}{2} D_{t} \\
& \tilde{D}_{t}=\frac{\beta u_{t}}{2 \omega \varepsilon_{t}} B_{t}+\frac{u_{t}}{2} D_{t} \\
& \tilde{E}_{t}=\frac{u_{t}}{2} A_{i}-\frac{\beta u_{t}}{2 \omega \mu} C_{t} \\
& \tilde{F}_{t}=\frac{u_{t}}{2} A_{t}+\frac{\beta u_{t}}{2 \omega \mu} C_{t} \\
& \tilde{G}_{t}=\frac{u_{t}}{2} B_{t}-\frac{\beta u_{t}}{2 \omega \mu} D_{t} \\
& \tilde{H}_{t}=\frac{u_{t}}{2} B_{i}+\frac{\beta u_{i}}{2 \omega \mu} D_{t}
\end{aligned}
$$

If $n_{e f f}>n_{i}$

$$
\begin{aligned}
& e_{r}=\left[\tilde{A}_{\imath} I_{v-1}\left(w_{\imath} r\right)-\tilde{B}_{\imath} I_{v+1}\left(w_{\imath} r\right)-\tilde{C}_{\imath} K_{v-1}\left(w_{t} r\right)-\tilde{D}_{\imath} K_{v+1}\left(w_{\imath} r\right)\right] \exp (j v \phi) \\
& e_{\phi}=j\left[\tilde{A}_{t} I_{v-1}\left(w_{\imath} r\right)+\tilde{B}_{\imath} I_{v+1}\left(w_{i} r\right)-\tilde{C}_{\imath} K_{v-1}\left(w_{\imath} r\right)+\tilde{D}_{t} K_{v+1}\left(w_{i} r\right)\right] \exp (j v \phi) \\
& h_{r}=j\left[\tilde{E}_{\imath} I_{v-1}\left(w_{t} r\right)-\tilde{F}_{\imath} I_{v+1}\left(w_{t} r\right)-\tilde{G}_{\imath} K_{v-1}\left(w_{\imath} r\right)-\tilde{H}_{\imath} K_{v+1}\left(w_{t} r\right)\right] \exp (j v \phi) \\
& h_{\phi}=\left[-\tilde{E}_{1} I_{v-1}\left(w_{t} r\right)-\tilde{F}_{\imath} I_{v+1}\left(w_{t} r\right)-\tilde{G}_{t} K_{v-1}\left(w_{t} r\right)-\tilde{H}_{t} K_{v+1}\left(w_{i} r\right)\right] \exp (j v \phi)
\end{aligned}
$$

Where

$$
\begin{aligned}
& \tilde{A}_{t}=-\frac{\beta w_{i}}{2 \omega \varepsilon_{i}} A_{t}+\frac{w_{t}}{2} C_{i} \\
& \tilde{B}_{t}=\frac{\beta w_{t}}{2 \omega \varepsilon_{t}} A_{t}+\frac{w_{t}}{2} C_{t}
\end{aligned}
$$




$$
\begin{aligned}
& \tilde{C}_{t}=-\frac{\beta w_{t}}{2 \omega \varepsilon_{t}} B_{t}+\frac{w_{t}}{2} D_{t} \\
& \tilde{D}_{i}=\frac{\beta w_{1}}{2 \omega \varepsilon_{t}} B_{t}+\frac{w_{t}}{2} D_{t} \\
& \tilde{E}_{t}=\frac{w_{t}}{2} A_{i}-\frac{\beta w_{t}}{2 \omega \mu} C_{t} \\
& \tilde{F}_{i}=\frac{w_{t}}{2} \Lambda_{i}+\frac{\beta w_{t}}{2 \omega \mu} C_{i} \\
& \tilde{G}_{t}=\frac{w_{t}}{2} B_{t}-\frac{\beta w_{t}}{2 \omega \mu} D_{t} \\
& \tilde{H}_{t}=\frac{w_{i}}{2} B_{t}+\frac{\beta w_{i}}{2 \omega \mu} D_{i}
\end{aligned}
$$

For the first layer $i=l$, we have $B_{l}=D_{l}=0$

$$
P_{z 1}=\operatorname{Re}\left[\frac{2 \pi}{u_{i}^{2}-\left(u_{t}^{*}\right)^{2}}\left(Q_{11}+Q_{12}\right)\right]
$$

Where

$$
\begin{aligned}
& Q_{11}=\left[u_{1}^{*} J_{0}\left(u_{1} a_{1}\right) J_{2}^{*}\left(u_{1} a_{1}\right)-u_{1} J_{0}^{*}\left(u_{1} a_{1}\right) J_{2}\left(u_{1} a_{1}\right)\right] \tilde{\Lambda}_{1} \tilde{E}_{1}^{*} \\
& Q_{12}=\left[u_{1} J_{1}\left(u_{1} a_{1}\right) J_{2}^{*}\left(u_{1} a_{1}\right)-u_{1}^{*} J_{1}^{*}\left(u_{1} a_{1}\right) J_{2}\left(u_{1} a_{1}\right)\right] \tilde{B}_{1} \tilde{F}_{1}^{*}
\end{aligned}
$$

For the layer $\mathrm{i}$ :

If $n_{\text {sff }}<n_{i}$

$$
P_{z i}=\operatorname{Re}\left[\frac{2 \pi}{u_{1}^{2}-\left(u_{1}^{*}\right)^{2}}\left(Q_{11}+Q_{12}+Q_{13}+Q_{14}+Q_{15}+Q_{i 6}+Q_{11}+Q_{i 8}\right)\right]
$$

Where 


$$
\begin{aligned}
& Q_{t 1}=\left[u_{i} a_{t} J_{v-2}\left(u_{t} a_{i}\right) J_{v-1}^{*}\left(u_{i} a_{t}\right)-u_{i}^{*} a_{t} J_{v-1}\left(u_{t} a_{i}\right) J_{v-2}^{*}\left(u_{i} a_{t}\right)\right. \\
& \left.-u_{t} a_{t-1} J_{v-2}\left(u_{t} a_{t-1}\right) J_{v-1}^{*}\left(u_{t} a_{t-1}\right)+u_{t}^{*} a_{t-1} J_{v-1}\left(u_{t} a_{t-1}\right) J_{v-2}^{*}\left(u_{t} a_{t-1}\right)\right] \tilde{A}_{i} \tilde{E}_{i}^{*} \\
& Q_{t 2}=\left[u_{t}^{*} a_{t} J_{v+1}\left(u_{t} a_{t}\right) J_{v}^{*}\left(u_{t} a_{t}\right)-u_{t} a_{t} J_{v}\left(u_{t} a_{t}\right) J_{v+1}^{*}\left(u_{t} a_{t}\right)\right. \\
& \left.-u_{t}^{*} a_{t-1} J_{v+1}\left(u_{t} a_{i-1}\right) J_{v}^{*}\left(u_{t} a_{i-1}\right)+u_{t} a_{i-1} J_{v}\left(u_{i} a_{i-1}\right) J_{v+1}^{*}\left(u_{i} a_{i-1}\right)\right] \tilde{B}_{t} \tilde{F}_{i}^{*} \\
& Q_{13}=\left[u_{t} a_{t} Y_{v-2}\left(u_{t} a_{t}\right) Y_{v-1}^{*}\left(u_{t} a_{t}\right)-u_{t}^{*} a_{i} Y_{v-1}\left(u_{t} a_{t}\right) Y_{v-2}^{*}\left(u_{i} a_{t}\right)\right. \\
& \left.-u_{t} a_{t-1} Y_{v-2}\left(u_{t} a_{t}\right) Y_{v-1}^{*}\left(u_{t} a_{t-1}\right)+u_{t}^{*} a_{t-1} Y_{v-1}\left(u_{t} a_{t-1}\right) Y_{v-2}^{*}\left(u_{i} a_{t-1}\right)\right] \tilde{C}_{t} \tilde{G}_{t}^{*} \\
& Q_{t 4}=\left[u_{t}^{*} a_{t} Y_{v+1}\left(u_{1} a_{1}\right) Y_{v}^{*}\left(u_{1} a_{1}\right)-u_{1} a_{1} Y_{v}\left(u_{1} a_{t}\right) Y_{v+1}^{*}\left(u_{t} a_{t}\right)\right. \\
& \left.-u_{t}^{*} a_{t-1} Y_{v+1}\left(u_{t} a_{t-1}\right) Y_{v}^{*}\left(u_{t} a_{t-1}\right)+u_{t} a_{t-1} Y_{v}\left(u_{t} a_{t-1}\right) Y_{v+1}^{*}\left(u_{i} a_{t-1}\right)\right] \tilde{D}_{i} \tilde{H}_{t}^{*} \\
& Q_{t 5}=\left[u_{1} a_{1} J_{v-2}\left(u_{t} a_{i}\right) Y_{v-1}^{*}\left(u_{i} a_{t}\right)-u_{t}^{*} a_{i} J_{v-1}\left(u_{i} a_{t}\right) Y_{v-2}^{*}\left(u_{t} a_{t}\right)\right. \\
& \left.-u_{t} a_{i-1} J_{v-2}\left(u_{t} a_{i-1}\right) Y_{v-1}^{*}\left(u_{i} a_{t-1}\right)+u_{i}^{*} a_{t-1} J_{v-1}\left(u_{t} a_{i-1}\right) Y_{v-2}^{*}\left(u_{i} a_{t-1}\right)\right] \tilde{A}_{t} \tilde{G} \\
& Q_{t 6}=\left[u_{t}^{*} a_{i} J_{v^{\prime}+1}\left(u_{t} a_{i}\right) Y_{v}^{*}\left(u_{t} a_{i}\right)-u_{t} a_{i} J_{v}\left(u_{t} a_{t}\right) Y_{t^{\prime}+1}^{*}\left(u_{i} a_{i}\right)\right. \\
& \left.-u_{t}^{*} a_{t-1} J_{v+1}\left(u_{t} a_{t-1}\right) Y_{v}^{*}\left(u_{t} a_{t-1}\right)+u_{t} a_{t-1} J_{v}\left(u_{t} a_{t-1}\right) Y_{v+1}^{*}\left(u_{i} a_{t-1}\right)\right] \tilde{B}_{t} \tilde{H}_{t}^{*} \\
& Q_{t 7}=\left[u_{t} a_{t} Y_{v-2}\left(u_{t} a_{i}\right) J_{v-1}^{*}\left(u_{i} a_{t}\right)-u_{t}^{*} a_{t} Y_{v-1}\left(u_{t} a_{t}\right) J_{v-2}^{*}\left(u, a_{t}\right)\right. \\
& \left.-u_{t} a_{t-1} Y_{v-2}\left(u_{t} a_{t}\right) J_{v-1}^{*}\left(u_{t} a_{t-1}\right)+u_{t}^{*} a_{t-1} Y_{v-1}\left(u_{t} a_{t-1}\right) J_{v-2}^{*}\left(u_{t} a_{t-1}\right)\right] \tilde{C}_{i} \tilde{E}_{t}^{*} \\
& Q_{18}=\left[u_{i}^{*} a_{t} Y_{v+1}\left(u_{i} a_{i}\right) J_{v}^{*}\left(u_{t} a_{i}\right)-u_{i} a_{i} Y_{v}\left(u, a_{t}\right) J_{v+1}^{*}\left(u_{i} a_{t}\right)\right. \\
& \left.-u_{t}^{*} a_{t-1} Y_{v+1}\left(u_{t} a_{t-1}\right) J_{v}^{*}\left(u_{t} a_{t-1}\right)+u_{t} a_{t-1} Y_{v}\left(u_{t} a_{t-1}\right) J_{v+1}^{*}\left(u_{t} a_{t-1}\right)\right] \tilde{D}_{i} \tilde{F}_{t}^{*}
\end{aligned}
$$

If $n_{c f f}>n_{t}$

$$
P_{i i}=\operatorname{Rc}\left[\frac{2 \pi}{w_{i}^{2}-\left(w_{1}\right)^{3}}\left(Q_{11}+Q_{12}+Q_{i 3}+Q_{i 4}+Q_{i 5}+Q_{16}+Q_{17}+Q_{i 8}\right)\right]
$$

Where 


$$
\begin{aligned}
Q_{l 1} & =\left[w_{\imath}^{*} a_{i} I_{v-1}\left(w_{i} a_{i}\right) I_{v-2}^{*}\left(w_{i} a_{\imath}\right)-w_{\imath} a_{\imath} I_{v-2}\left(w_{i} a_{t}\right) I_{v-1}^{*}\left(w_{i} a_{i}\right)\right. \\
& \left.-w_{t}^{*} a_{t-1} I_{v-1}\left(w_{t} a_{t-1}\right) I_{v-2}^{*}\left(w_{\imath} a_{t-1}\right)+w_{\imath} a_{t-1} I_{v-2}\left(w_{t-1} a_{\imath}\right) I_{v-1}^{*}\left(w_{\imath} a_{t-1}\right)\right] \tilde{A}_{\imath} \tilde{E}_{i}^{*}
\end{aligned}
$$

$$
\begin{aligned}
Q_{t 2}= & {\left[w_{t} a_{\imath} I_{v}\left(w_{i} a_{t}\right) I_{v+1}^{*}\left(w_{t} a_{t}\right)-w_{i}^{*} a_{t} I_{v+1}\left(w_{t} a_{i}\right) I_{v}^{*}\left(w_{i} a_{i}\right)\right.} \\
& \left.-w_{i} a_{i-1} I_{v}\left(w_{i} a_{i-1}\right) I_{v+1}^{*}\left(w_{\imath} a_{t-1}\right)+w_{t}^{*} a_{i-1} I_{v+1}\left(w_{i} a_{t-1}\right) I_{v}^{*}\left(w_{\imath} a_{i-1}\right)\right] \tilde{B}_{i} \tilde{F}_{t}^{*}
\end{aligned}
$$

$$
\begin{aligned}
Q_{t 3} & =\left[w_{\imath} a_{\imath} K_{v-2}\left(w_{t} a_{\imath}\right) K_{v-1}^{*}\left(w_{t} a_{t}\right)-w_{t}^{*} a_{\imath} K_{v-1}\left(w_{t} a_{t}\right) K_{v-2}^{*}\left(w_{t} a_{t}\right)\right. \\
& \left.-w_{\imath} a_{t-1} K_{v-2}\left(w_{i} a_{i-1}\right) K_{v-1}^{*}\left(w_{i} a_{i-1}\right)+w_{t}^{*} a_{i-1} K_{v-1}\left(w_{\imath} a_{i-1}\right) K_{v-2}^{*}\left(w_{\imath} a_{i-1}\right)\right] \tilde{C}_{\imath} \tilde{G}_{t}^{*}
\end{aligned}
$$

$$
\begin{aligned}
Q_{i+} & =\left[w_{i}^{*} a_{i} K_{v+1}\left(w_{i} a_{i}\right) K_{v}^{*}\left(w_{i} a_{i}\right)-w_{i} a_{i} K_{v}\left(w_{\imath} a_{t}\right) K_{v+1}^{*}\left(w_{t} a_{t}\right)\right. \\
& \left.-w_{t}^{*} a_{t-1} K_{v+1}\left(w_{t} a_{t-1}\right) K_{v}^{*}\left(w_{t} a_{i-1}\right)+w_{\imath} a_{t-1} K_{v}\left(w_{t} a_{t-1}\right) K_{v+1}^{*}\left(w_{\imath} a_{t-1}\right)\right] \tilde{D}_{\imath} \tilde{H}_{t}^{*}
\end{aligned}
$$

$$
\begin{aligned}
Q_{t 5}= & {\left[w_{t}^{*} a_{t} I_{v-1}\left(w_{t} a_{t}\right) K_{v-2}^{*}\left(w_{t} a_{t}\right)+w_{i} a_{t} I_{v-2}\left(w_{t} a_{t}\right) K_{v-1}^{*}\left(w_{t} a_{i}\right)\right.} \\
& \left.-w_{t}^{*} a_{t-1} I_{v-1}\left(w_{i} a_{t-1}\right) K_{v-2}^{*}\left(w_{t} a_{t-1}\right)-w_{t} a_{t-1} I_{v-2}\left(w_{t} a_{i-1}\right) K_{i-1}^{*}\left(w_{i} a_{i-1}\right)\right] \tilde{A}_{i} \tilde{G}_{t}^{*}
\end{aligned}
$$$$
Q_{10}=\left[w_{t}^{*} a_{i} I_{v+1}\left(w_{t} a_{t}\right) K_{v}^{*}\left(w_{t} a_{t}\right)+w_{t} a_{t} I_{v}\left(w_{i} a_{i}\right) K_{v+1}^{*}\left(w_{i} a_{t}\right)\right.
$$$$
\left.-w_{i}^{*} a_{t-1} I_{v+1}\left(w_{t} a_{t-1}\right) K_{v}^{*}\left(w_{i} a_{t-1}\right)-w_{t} a_{t-1} I_{v}\left(w_{t} a_{t-1}\right) K_{v+1}^{*}\left(w_{t} a_{t-1}\right)\right] \tilde{B}_{i} \tilde{H}_{i}^{*}
$$

$$
\begin{aligned}
Q_{\imath 7} & =\left[w_{t} a_{i} K_{v-2}\left(w_{i} a_{i}\right) I_{v-1}^{*}\left(w_{t} a_{t}\right)+w_{\imath}^{*} a_{i} K_{v-1}\left(w_{i} a_{i}\right) I_{v-2}^{*}\left(w_{i} a_{t}\right)\right. \\
& \left.-w_{\imath} a_{i-1} K_{v-2}\left(a_{t}\right) I_{v-1}^{*}\left(w_{\imath} a_{t-1}\right)-w_{t}^{*} a_{t-1} K_{v-1}\left(w_{t} a_{i-1}\right) I_{v-2}^{*}\left(w_{\imath} a_{i-1}\right)\right] \tilde{C}_{i} \tilde{E}_{t}^{*}
\end{aligned}
$$

$$
\begin{aligned}
Q_{18} & =\left[w_{t}^{*} a_{i} K_{v+1}\left(w_{t} a_{t}\right) I_{v}^{*}\left(w_{t} a_{t}\right)+w_{t} a_{t} K_{v}\left(w_{t} a_{t}\right) I_{v+1}^{*}\left(w_{i} a_{t}\right)\right. \\
& \left.-w_{i}^{*} a_{i-1} K_{v+1}\left(w_{t} a_{t-1}\right) I_{v}^{*}\left(w_{i} a_{t-1}\right)-w_{i} a_{i-1} K_{v}\left(w_{t} a_{t-1}\right) I_{v+1}^{*}\left(w_{i} a_{i-1}\right)\right] \tilde{D}_{t} \tilde{F}_{t}^{*}
\end{aligned}
$$

For the last layer $i=N$, we have $A_{N}=C_{N}=0$,

$$
P_{z N}=\operatorname{Re}\left[\frac{2 \pi a_{N-1}}{w_{N}^{2}-\left(w_{N}^{*}\right)^{2}}\left(Q_{N 3}+Q_{N 4}\right)\right]
$$

Where

$$
Q_{N 3}=\left[w_{N}^{*} K_{v-1}\left(w_{N} a_{N-1}\right) K_{v-2}^{*}\left(w_{N} a_{N-1}\right)-w_{N} K_{v-2}^{*}\left(w_{N} a_{N-1}\right) K_{v-1}^{*}\left(w_{N} \iota_{N-1}\right)\right] \tilde{C}_{N} \tilde{G}_{N}^{*}
$$




$$
Q_{N+}=\left[w_{N} K_{v}\left(w_{N} a_{N-1}\right) K_{v+1}^{*}\left(w_{N} a_{N-1}\right)-w_{N}^{*} K_{v+1}\left(w_{N} a_{N-1}\right) K_{v}^{*}\left(w_{N} a_{N-1}\right)\right] \tilde{D}_{N} \tilde{H}_{N}^{*}
$$

The total power in the fibre

$$
P=\sum_{i=1}^{N} P_{z t}
$$

By forcing the $P=1 \mathrm{~W}$, we can obtain normalized field coefficients $A, B, C$ and $D$ and normaiized field distributions. It is important to have normalized fields when doing the mode coupling calculations and comparing field distributions between different modes. 


\section{Appendix B: Publication List}

\section{B1. Patents}

J. Albert, C. Chen, Y. Shevchenko and A. Ivanov, "Tilted grating sensor", International Patent Application WO/2008/049187

\section{B2. Referred Journal Papers}

1. C. Chen, S. Dods, and J. Albert, "Efficient and accurate analysis of fibre modes in multilayer step-index fibres with lossy and gain media," paper in process.

2. C. Chen and J. Albert, "Analysis of cladding mode resonances in Tilted FBG in optical fibres with a thin metal coating for high index sensitivity biomedical sensor applications," paper in process.

3. C. Caucheteur, S. Bette, C. Chen, M. Wuilpart, P. Mégret, and J. Albert, "Tilted Fibre Bragg Grating Refractometer Using Polarization Dependent Loss Measurement," accepted for publication in IEEE Photon. Technol. Lett., 2008.

4. C. Caucheteur, M. Wuilpart, C. Chen, P. Mégret, and J. Albert, "Quasidistributed refractometer using tilted Bragg gratings and time domain reflectometry," Opt. Express, vol. 16, pp.17882-17890, 2008.

5. C. Chen, A. Laronche1, G. Bouwmans, L. Bigot, Y. Quiquempois, and J. Albert, "Sensitivity of photonic crystal fibre modes to temperature, strain and external refractive index," Opt. Express. vol. 16, pp. 9645-9653, 2008.

6. X. Dai, R. B. Walker, S. J. Mihailov, C. Chen, C. Blanchetière, C. L. Callender, and J. Albert, "Temperature insensitive refractometer using core and cladding modes in open-top ridge waveguide," IEEE Sensors Journal , vol. 8, pp451-456, 2008 
7. T. Guo, A. Ivanov, C. Chen, and J. Albert, "Temperature-independent tilted fibre grating vibration sensor based on cladding-core recoupling," Optics Lett. vol. 33, pp.1004-1006, 2008.

8. T. Guo, C. Chen, A. Laronche, and J. Albert, "Power-referenced and tenı.erstire-calibrated optical fibre refractometer," IEEE Photon. Technol. Lett. vol. $20, \mathrm{pp} .635-637,2008$.

9. C. Chen, C. Caucheteur, P. Mégret, and J. Albert, "The sensitivity characteristics of tilted fibre Bragg grating sensors with different cladding thicknesses," Meas Sci. \&Techn., vol. 18, pp. 3117-3122, 2007.

10. C. F. Chan, C. Chen, A. Jafari, A. Laronche, D. J. Thomson and J. Albert, "Optical fibre refractometer using narrowband cladding mode resonance shifts," Appl. Optics, vol. 46, pp. 1142-1149, 2007.

11. C. Chen and J. Albert, "Strain-optic coefficients of the individual cladding modes of a single mode fibre: theory and experiment," Electron. Lett. , vol. 48, pp. 1027-1028, 2006.

\section{B3. Book Chapters}

C. Chen, Y. Shevchenko and J. Albert, Novel sensing mechanism using tilted fibre Bragg gratings, Chapter 2 in Optical Waveguide Sensing and Imaging, W. J. Bock, I. Gannot and S. Tanev (ed.), Springer, Netherlands, 2008

\section{B4. Refereed Conference Papers}

12. C. Caucheteur, S. Bette, C. Chen, M. Wuilpart, P. Mégret, and J. Albert, "Refractometer based on polarization dependent loss measurement of weakly tilted fibrc bragg gratings," 14th Microoptics Conference, paper P-54, Brussels Belgium, 2008.

13. C. Chen, A. Jaronche, G. Bouwmans, L. Bigot, Y'. Quiquempois, and J. Albert, "The inner cladding mode in a photonic crystal fibre for temperatureand 
refractive index-independent strain sensing applications." $19^{\text {th }}$ Conference on Optical Fibre Sensors, Proc. SPIE 7004, 70046B pp. 1-4, 2008.

14. C. Chen, T. Guo, A. Laronche, and J. Albert, "Radiation mode resonances of tilted fibre Bragg gratings for high index media measurement," $19^{\text {th }}$ Conference on Optical Fibre Sensors ,Proc. SPIE 7004, 700418 pp. 1-4, 2008.

15. T. Guo, J. Albert, C. Chen, A. Ivanov, and A. Laronche, "Highly accurate microdisplacement measurement based on Gaussian-chirped tilted fibre Bragg grating," $19^{\text {th }}$ Conference on Optical Fibre Sensors ,Proc. SPIE 7004, 700417 pp. 1-4, 2008.

16. C. Caucheteur, C. Chen, J. Albert, and P. Mégret, "Weakly tilted fibre Bragg gratings for sensing purposes(Invited paper)," Photonics Europe, Proc. SPIE 7003, 700307 pp. 1-12, 2008.

17. C. Chen and J. Albert, "Analysis of plasmon-like modes in optical fibre with a nano-size gold Overlay for biochemical sensor applications," OSA Topical Conference on Nanophotonics, paper Nano4.5, Hangzhou China, June 2007.

18. X. Dai, S. J. Mihailov, R. B. Walker, C. Chen, and J. Albert, "Design of high sensitivity refractometer based on temperature independent TE and TMT modes in open top ridge waveguides," Proc. SPIE 6770, 67700B 1-6, 2007.

19. C. Chen and J. Albert, "Hybrid modes analysis for cladding mode resonance peaks in tilted fibre Bragg gratings," Proc. SPIE 6796, 679623 pp. 1-6, 2007.

20. Y. Shevchenko, A. Ianoul, C. Chen, and J. Albert, "Realization of Surface Plasmon Resonance Sensor in Standard Optical Fibres," Proc. of SPIE 6796, 67961Z1-12, 2007.

21. C. F. Chan, G. A. Ferrier, D. J. Thomson, C. Chen, J. Albert, P. Lefebvre, and A. Vincelette, "Side-polished and tilted fibre Bragg grating sensors for structurai health monitoring applications," Proc. SPIE 6530, 65300F1-10, 2007.

22. C. Chen, C. Caucheteur, P. Mégrel, and J. Albert, "Sensitivity of tilted fibre Bragg grating sensors with different cladding thicknesses", $18^{\text {th }}$ Conference on Optical Fibre Sensors, paper TuE31, 2006. 
23. C. Chen, L. Xiong, C. Caucheteur, P. Mégret, and J. Albert, "Differential strain sensitivity of higher order cladding modes in weakly tilted fibre Bragg gratings," Proc. SPIE 6379. 63790E1-7, 2006.

24. C. Caucheteur, C. Chen, J. Albert, P. Mégret, "Use of weakly titled fibre Bragg gratings for strain sensing purposes," Proc. Symposium IEEE/LEOS Benelux Chapter, pp. 61-64, 2006.

25. C. Caucheteur, C. Chen, J. Albert. and P. Mégret, "Utilisation des réseaux de Bragg fibrés faiblement inclinés des fins de capteurs multiparamétriques," Journées Nationales d'Optique Guidée, JNOG 2006, Metz, France, 2006.

26. C. Chen, L. Xiong, A. Jafari, and J. Albert, "Differential sensitivity characteristics of tilted fibre Bragg grating sensors", Proc. SPIE 6004, 60040B1$10,2005$.

27. C. Chen and J. Albert, "Photo-induced signal taps for power monitors in planar lightwave circuits", Proc. SPIE 5970, 5970011-8, 2005. 